COMPILATION OF GROUND-WATER LEVEL MEASUREMENTS OBTAINED BY THE UNITED STATES GEOLOGICAL SURVEY IN PUERTO RICO, 1958-1985

By Sigfredo Torres-González

U.S. GEOLOGICAL SURVEY

Open-File Data Report 88-701

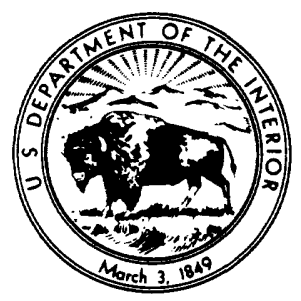

San Juan, Puerto Rico 1991 


\section{U.S. DEPARTMENT OF THE INTERIOR \\ MANUEL LUJAN, JR., Secretary \\ U.S. GEOLOGICAL SURVEY \\ Dallas L. Peck, Director}

For additional information write to:

District Chief

U.S. Geological Survey

P.O. Box 364424

San Juan, Puerto Rico 00936-4424
Copies of this report can be purchased from:

U.S. Geological Survey

Books and Open-File Reports

Federal Center, Bldg. 810

Box 25425, Denver, CO 80225 


\section{CONTENTS}

Page

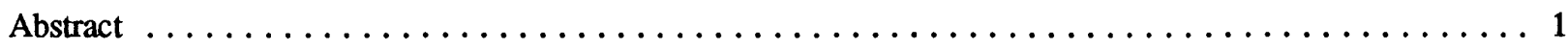

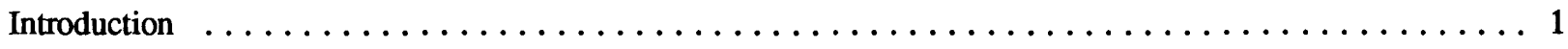

Explanation of data presented $\ldots \ldots \ldots \ldots \ldots \ldots \ldots \ldots \ldots \ldots \ldots \ldots \ldots \ldots \ldots \ldots \ldots$

Compilation of ground-water level measurements $\ldots \ldots \ldots \ldots \ldots \ldots \ldots \ldots \ldots \ldots \ldots \ldots$

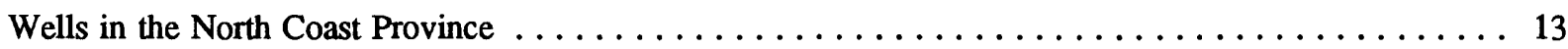

West Coast to Río Grande de Arecibo $\ldots \ldots \ldots \ldots \ldots \ldots \ldots \ldots \ldots \ldots \ldots \ldots \ldots$

Río Grande de Arecibo to Río de la Plata $\ldots \ldots \ldots \ldots \ldots \ldots \ldots \ldots \ldots \ldots \ldots \ldots$

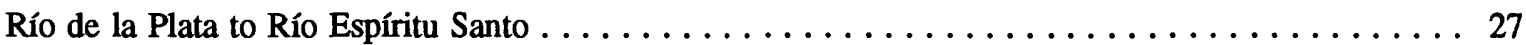

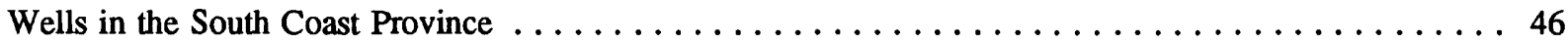

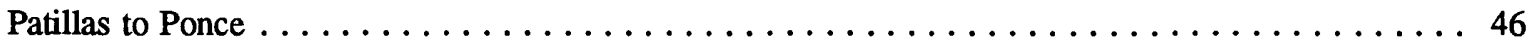

Tallaboa-Guayanilla-Yauco-Guánica $\ldots \ldots \ldots \ldots \ldots \ldots \ldots \ldots \ldots \ldots \ldots \ldots \ldots \ldots$

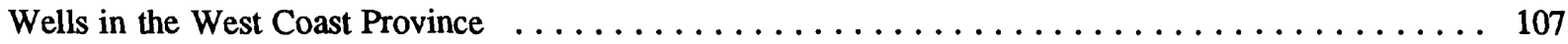

Río Guanajibo Valley $\ldots \ldots \ldots \ldots \ldots \ldots \ldots \ldots \ldots \ldots \ldots \ldots \ldots \ldots \ldots \ldots \ldots \ldots$

Río Yagüez Valley $\ldots \ldots \ldots \ldots \ldots \ldots \ldots \ldots \ldots \ldots \ldots \ldots \ldots \ldots \ldots \ldots \ldots \ldots \ldots \ldots \ldots \ldots$

Río Grande de Añasco Valley $\ldots \ldots \ldots \ldots \ldots \ldots \ldots \ldots \ldots \ldots \ldots \ldots \ldots \ldots \ldots \ldots \ldots$

Río Culebrina Valley $\ldots \ldots \ldots \ldots \ldots \ldots \ldots \ldots \ldots \ldots \ldots \ldots \ldots \ldots \ldots \ldots$

Wells in the East Coast Province $\ldots \ldots \ldots \ldots \ldots \ldots \ldots \ldots \ldots \ldots \ldots \ldots \ldots \ldots \ldots$

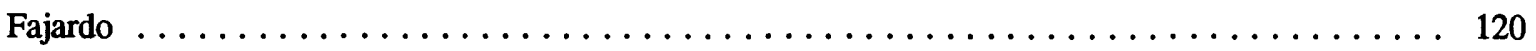

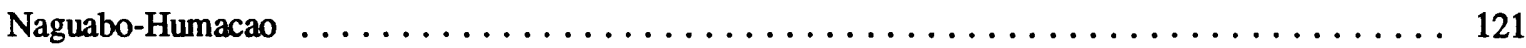

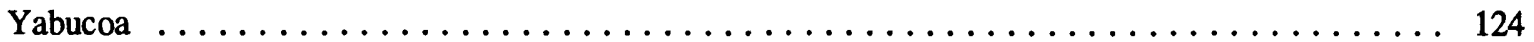

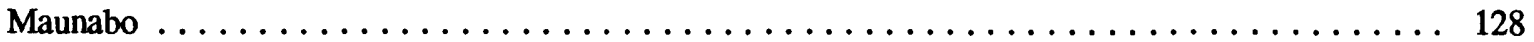

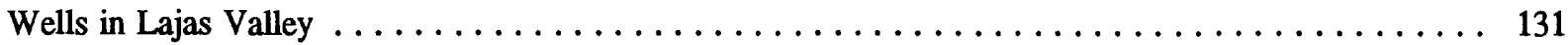

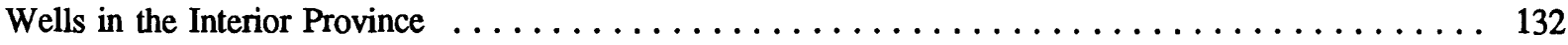

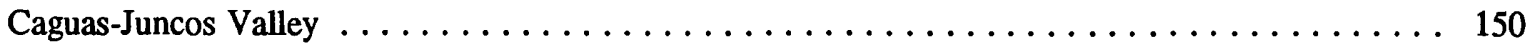

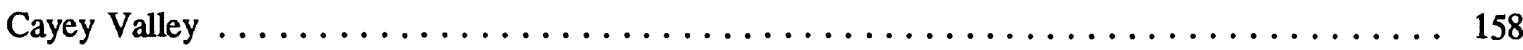

Selected references $\ldots \ldots \ldots \ldots \ldots \ldots \ldots \ldots \ldots \ldots \ldots \ldots \ldots \ldots \ldots \ldots \ldots \ldots \ldots \ldots \ldots$ 


\section{ILLUSTRATIONS}

Page

Figure

1. Map showing aquifer areas and sub areas in Puerto Rico $\ldots \ldots \ldots \ldots \ldots \ldots \ldots \ldots \ldots \ldots$

2. Map showing general location of observation wells in Puerto Rico $\ldots \ldots \ldots \ldots \ldots \ldots \ldots \ldots$

3-48. Graphs showing ground-water levels at:

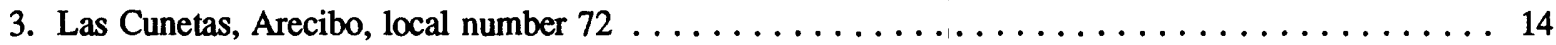

4. Montebello, local number $56 \ldots \ldots \ldots \ldots \ldots \ldots \ldots \ldots \ldots \ldots \ldots \ldots \ldots$

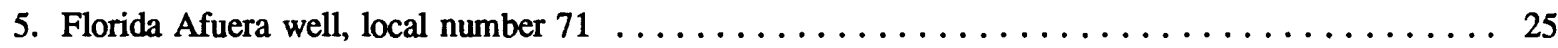

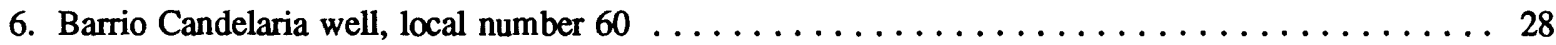

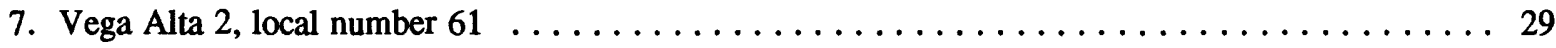

8. Vega Alta Number 1 , local number $62 \ldots \ldots \ldots \ldots \ldots \ldots \ldots \ldots \ldots \ldots \ldots \ldots \ldots \ldots$

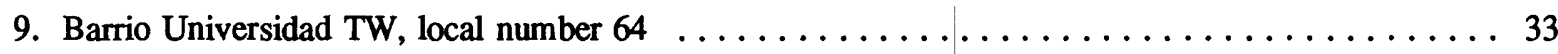

10. Hato Rey Central, McCracken, local number $65 \ldots \ldots \ldots \ldots \ldots \ldots \ldots \ldots \ldots \ldots \ldots$

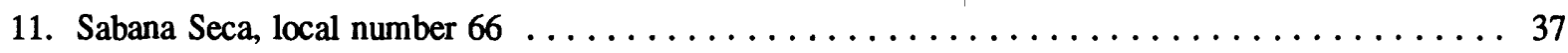

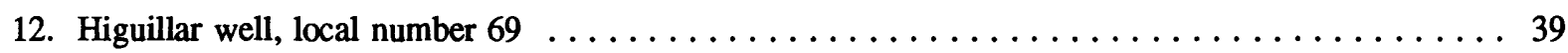

13. Barrio Sabana Hoyos, local number $70 \ldots \ldots \ldots \ldots \ldots \ldots \ldots \ldots \ldots \ldots \ldots \ldots \ldots \ldots \ldots \ldots \ldots$

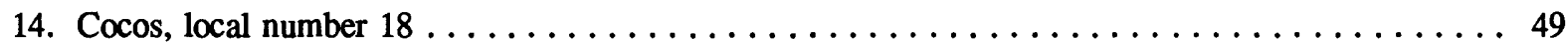

15. Theater number 1 , local number $19 \ldots \ldots \ldots \ldots \ldots \ldots \ldots \ldots \ldots \ldots \ldots \ldots \ldots \ldots$

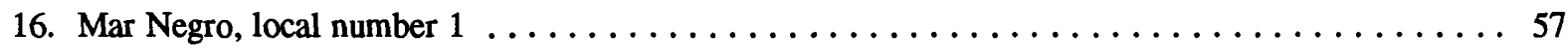

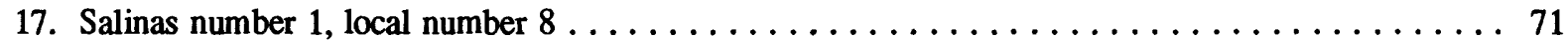

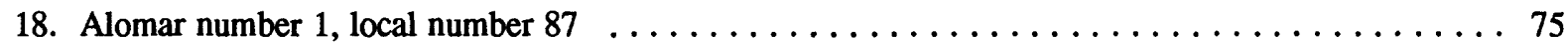

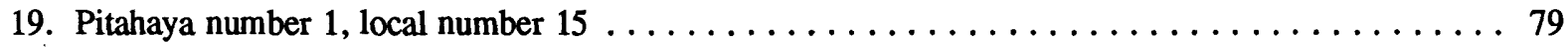

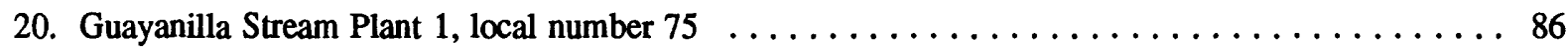

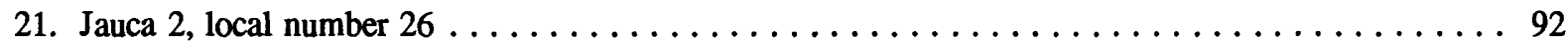

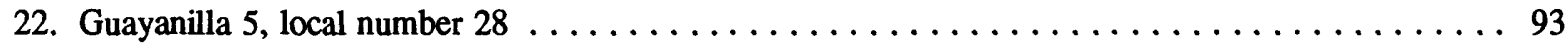

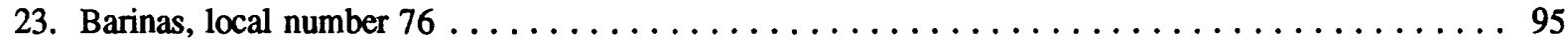

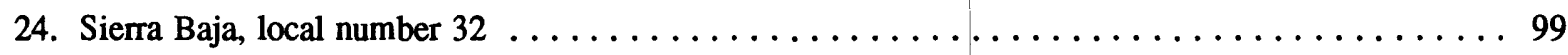

25. Rosario, local number $40 \ldots \ldots \ldots \ldots \ldots \ldots \ldots \ldots \ldots \ldots \ldots$

26. Central Rochelaise, local number $43 \ldots \ldots \ldots \ldots \ldots \ldots \ldots \ldots \ldots \ldots \ldots \ldots$

27. Mayagüez, local number $44 \ldots \ldots \ldots \ldots \ldots \ldots \ldots \ldots \ldots \ldots \ldots \ldots \ldots \ldots \ldots$

28. Well 1, Mayagüez, local number $45 \ldots \ldots \ldots \ldots \ldots \ldots \ldots \ldots \ldots \ldots \ldots \ldots \ldots$

29. Mayagüez Airport, local number $53 \ldots \ldots \ldots \ldots \ldots \ldots \ldots \ldots \ldots \ldots \ldots \ldots \ldots$

30. Aguada, local number $58 \ldots \ldots \ldots \ldots \ldots \ldots \ldots \ldots \ldots \ldots \ldots \ldots \ldots \ldots \ldots$ 


\section{ILLUSTRATIONS---Continued}

Figure

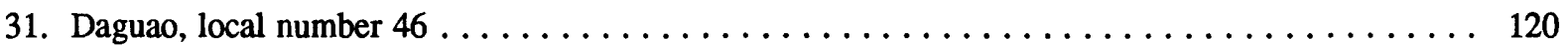

32. Río Humacao ground-water station, local number $172 \ldots \ldots \ldots \ldots \ldots \ldots \ldots \ldots \ldots$

33. Squibb observation well number 3, local number $173 \ldots \ldots \ldots \ldots \ldots \ldots \ldots \ldots \ldots$

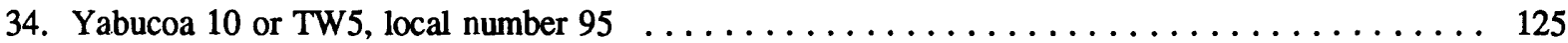

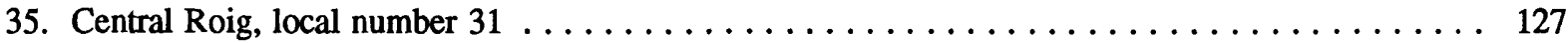

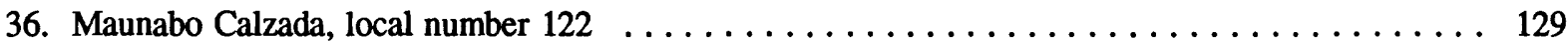

37. Vivoni, Hacienda Amistad, local number $143 \ldots \ldots \ldots \ldots \ldots \ldots \ldots \ldots \ldots \ldots \ldots$

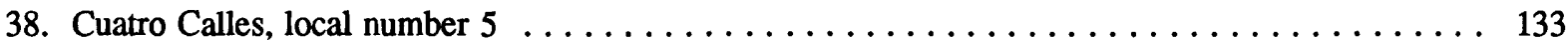

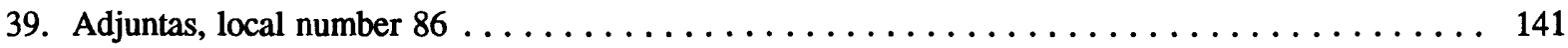

40. Piñas 1 (Comerío), local number $47 \ldots \ldots \ldots \ldots \ldots \ldots \ldots \ldots \ldots \ldots \ldots \ldots$

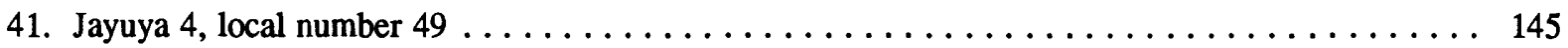

42. Bayaney, local number $57 \ldots \ldots \ldots \ldots \ldots \ldots \ldots \ldots \ldots \ldots \ldots \ldots \ldots$

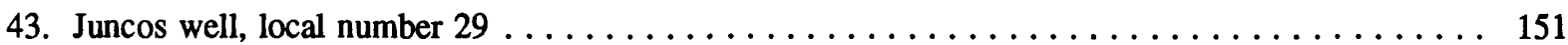

44. Gurabo Experimental Station, local number $50 \ldots \ldots \ldots \ldots \ldots \ldots \ldots \ldots \ldots \ldots$

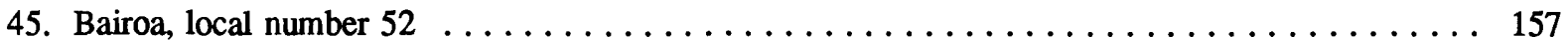

46. Cayey 10 , local number $33 \ldots \ldots \ldots \ldots \ldots \ldots \ldots \ldots \ldots \ldots \ldots \ldots \ldots \ldots$

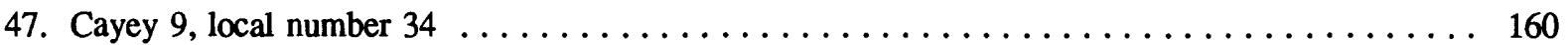

48. Cayey Mogate, local number $35 \ldots \ldots \ldots \ldots \ldots \ldots \ldots \ldots \ldots \ldots \ldots \ldots \ldots \ldots \ldots$

\section{TABLES}

Table

1. Location and identification of selected wells in Puerto Rico, 


\section{CONVERSION FACTORS}

For the convenience of readers who may want to use International System units (SI), the data may be converted by using the following factors:

Multiply inch-pound units

inch (in.)

feet (ft)
By

25.4

0.3048
To obtain SI units

millimeter (mm)

meter $(\mathrm{m})$ 


\title{
COMPILATION OF GROUND-WATER LEVEL MEASUREMENTS OBTAINED BY THE UNITED STATES GEOLOGICAL SURVEY IN PUERTO RICO, 1958-1985
}

\author{
By Sigfredo Torres-González
}

\begin{abstract}
A digital compilation of ground-water levels in Puerto Rico was prepared as part of the Caribbean Islands Regional Aquifer-System Analysis program. Of special interest are the ground-water level measurements obtained on a routine basis at wells located in different aquifer areas or provinces. Data from 181 observation wells were entered in a computer data base. The data base includes the following: well name, site identification number, aquifer area, depth of well, local number, local office number, year of record, construction date, earliest ground-water level and date reported, owner, diameter of well, and ground-water level fluctuations for various time periods between 1958 and 1985. The data include the conditions which may have affected ground-water level measurements. If several ground-water level mesurements are available during a month the lowest water level was reported.
\end{abstract}

\section{INTRODUCTION}

The U.S. Geological Survey (USGS) has routinely collected data on ground-water levels at observation wells in different aquifer areas in Puerto Rico since 1958 (fig. 1). The information is available to the public in printed format on a yearly basis as part of the Water Resources Data publication series. To make the information more accessible, the data were compiled, reformatted, and entered into a computer data base. The data presented in this report provides ground-water level measurements for 181 wells between 1958 and 1985. An important element of the Caribbean Islands Regional Aquifer System-Analysis (RASA) program is the compilation of data related to the island aquifers into automated data bases. Of special interest are the water-level measurements routinely obtained at observation wells. The water level of wells selected for routine observations represent the water levels of the aquifer regions of Puerto Rico.

\section{EXPLANATION OF DATA PRESENTED}

Ground-water level measurements from 181 observation wells in Puerto Rico are compiled and presented in this report. In table 1 , wells are identified by name and organized according to aquifer area. The following information is provided: the USGS site identification number, aquifer area (refer to fig. 1), depth of well, local number (fig. 2), local office number, years of available records, construction date, and the earliest water level reported. 


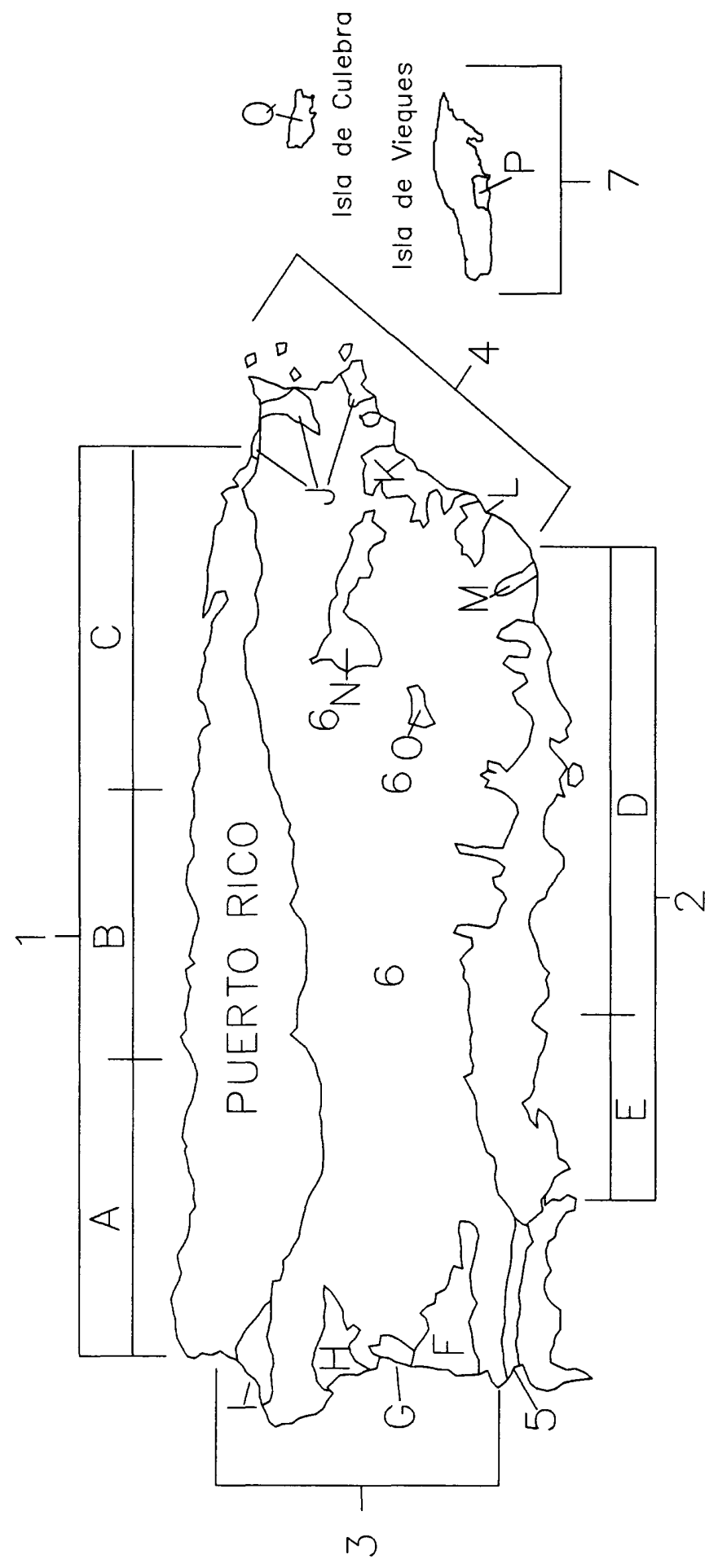

离

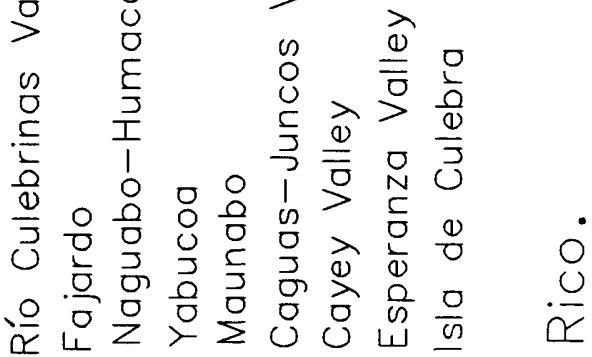

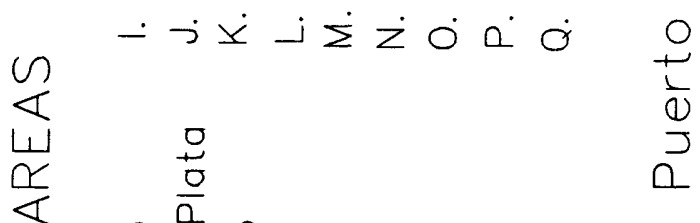

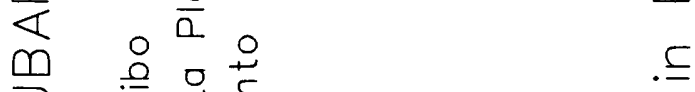

ज)

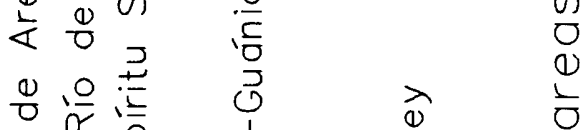

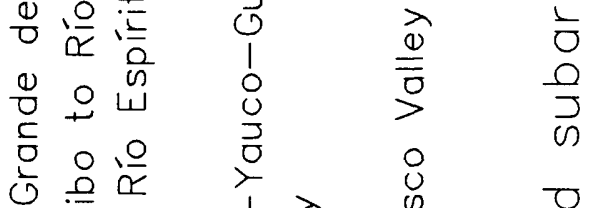

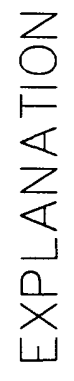

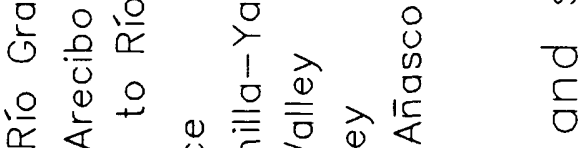

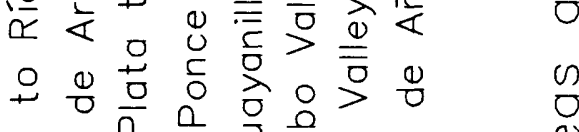

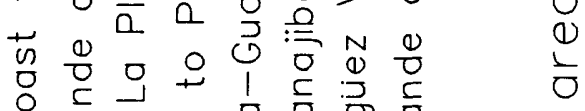

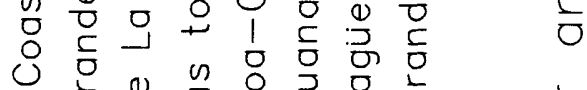

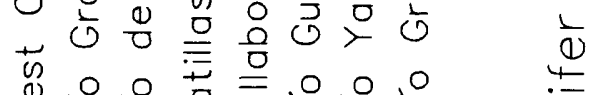

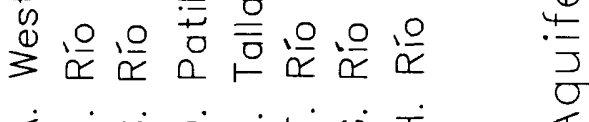

く்́

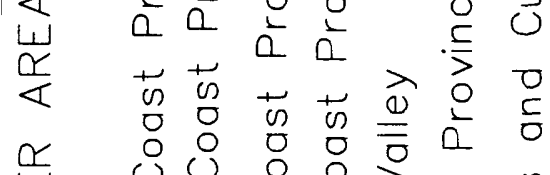

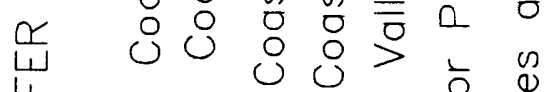

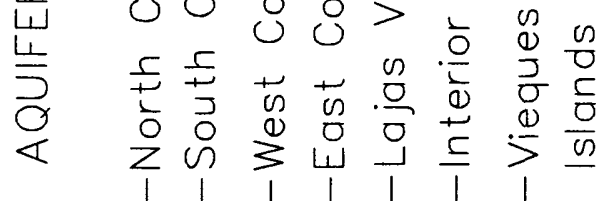

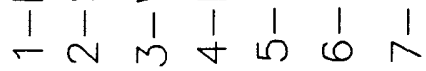




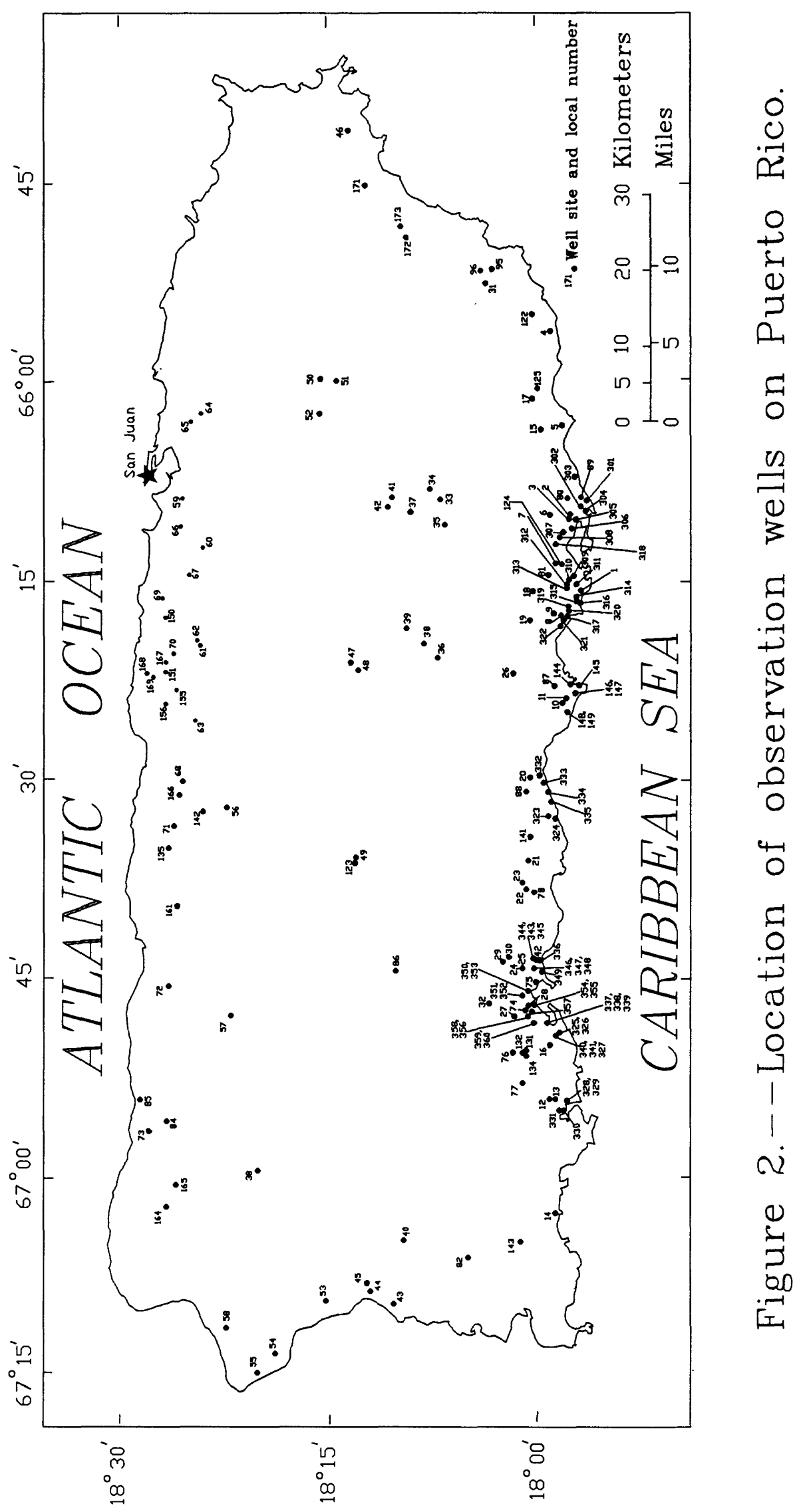


The USGS site identification number is based on the grid system of latitude and longitude. This system provides the geographic location of the well and a unique number for each site. The number consists of 15 digits. The first 6 digits denote the degrees, minutes, and seconds of latitude; the next 7 digits denote degrees, minutes, and seconds longitude; and the last 2 digits (assigned sequentially) identify the wells within a 1-second grid. The local number is a number given to wells sequentially as they were added to the observation well network. The local numbers are shown in figure 2 .

The local office number was used to identify well sites in the past, but has been replaced by the site identification number. The local office number, 28-66.56-01-077, contains four parts:

28 -- $\quad$ is the minute of latitude of the southeast corner of the 1-minute quadrilateral in which the well is located. The degrees of latitude have been omitted because most of the island of Puerto Rico lies between 18 degrees 00 minutes and 18 degrees 30 minutes.
66.56 -- is the degree and minute of longitude of the southeast corner of this quadrangle.
$01 \quad--\quad$ is an arbitrary number representing the order in which the well, within the quadrilateral, was inventoried.
77 -- is the number that identifies one of 100 small rectangles within the 1-minute quadrilateral, in which the well is located. The rectangles are numbered from left to right starting with the upper tier and proceeding, left to right, through each tier of rectangles to the lowest tier.

A compilation of water-level measurements is presented in feet, for each month, for the years for which records are available. The data are arranged by well in order of aquifer area. The conditions under which these measurements were taken are indicated. Depth to water measurements were in many types of wells, such as domestic, public water supply, or observation well, either active or abandoned, and under varying conditions (static, pumping, and recently pumped); however, the equipment and techniques used ensured that measurements made at each well were consistent under a given set of conditions.

Water-level measurements are given in feet with reference either to mean sea level (MSL) or land-surface datum (LSD). For the wells that had several monthly measurements, the lowest water level (represented by the greatest depth to water) was reported. Negative water-level measurements indicate a piezometric head higher than LSD or MSL. The blank spaces in the compilation indicate that data were not available for a given month. Hydrographs of ground-water levels from representative wells in each aquifer area show the long-term water level trend (figs. 3 through 48). 


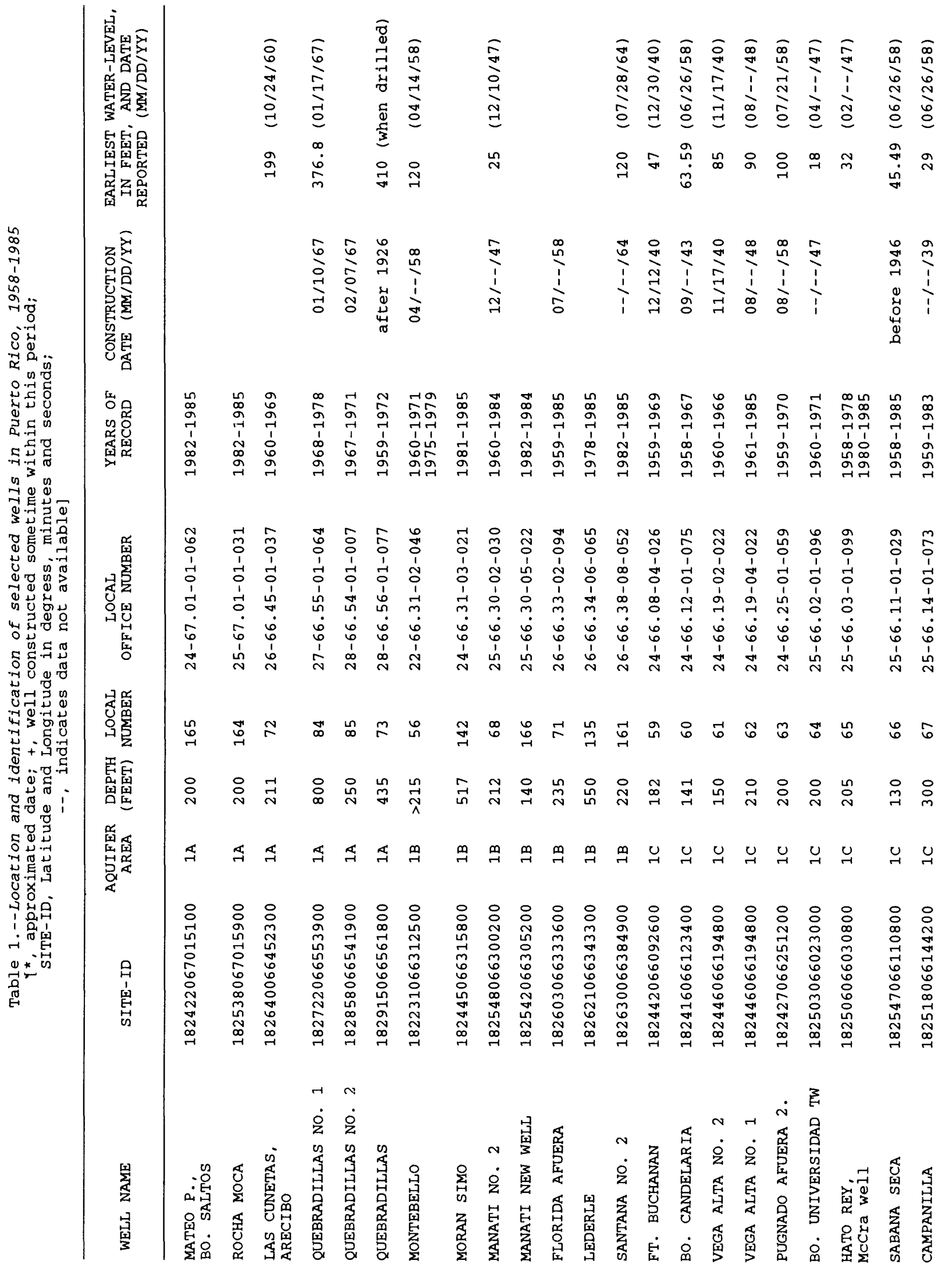




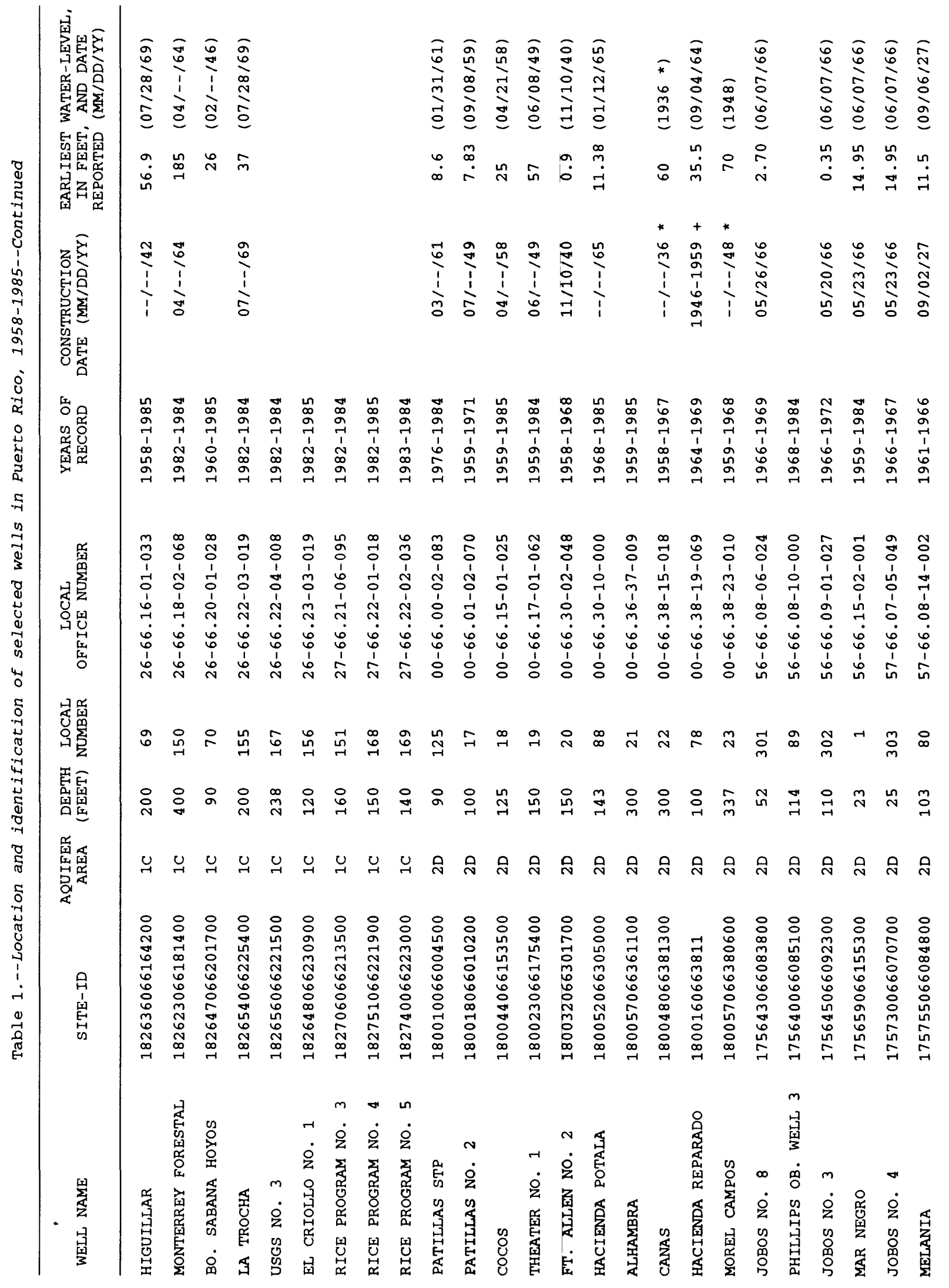




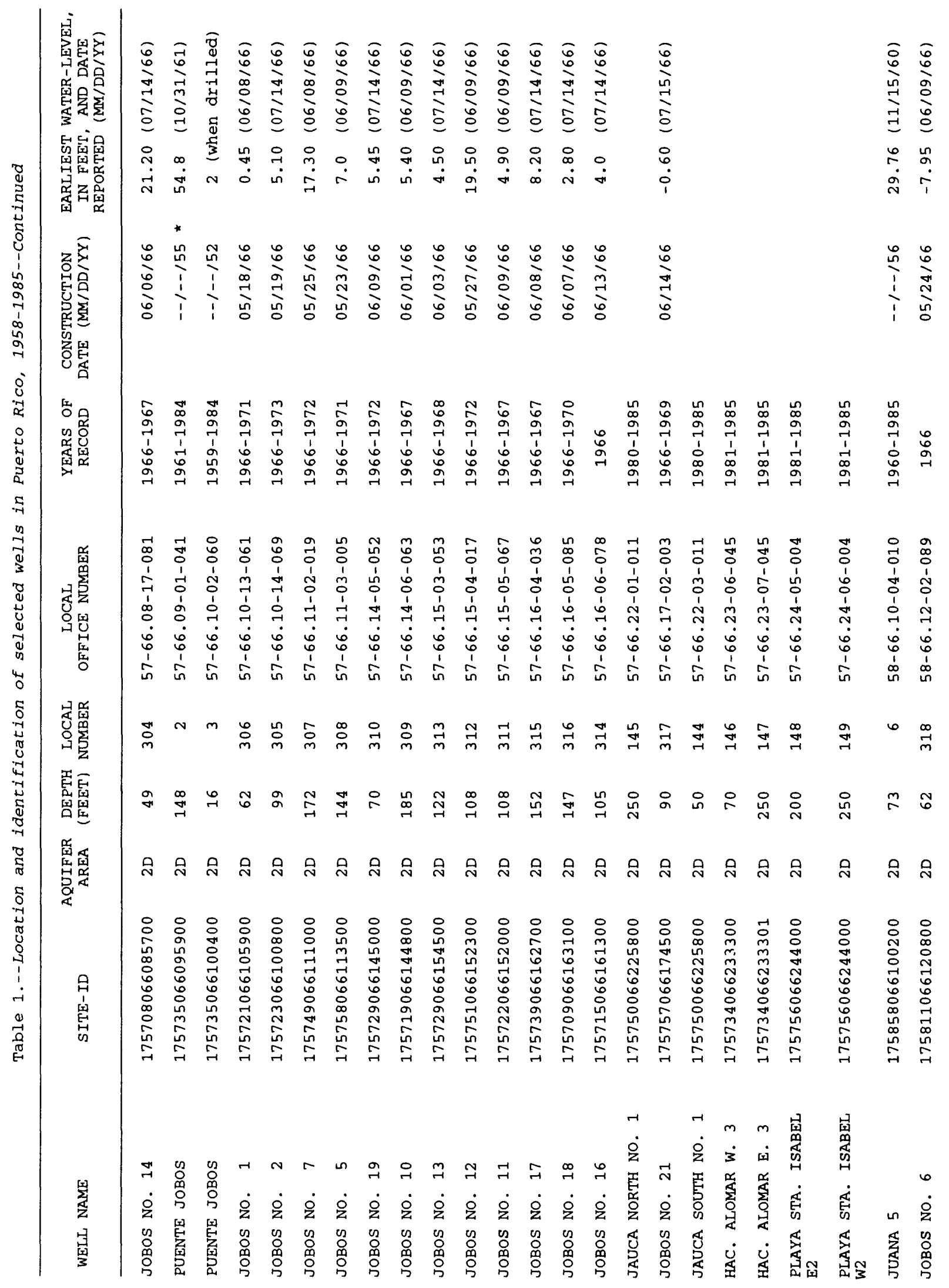




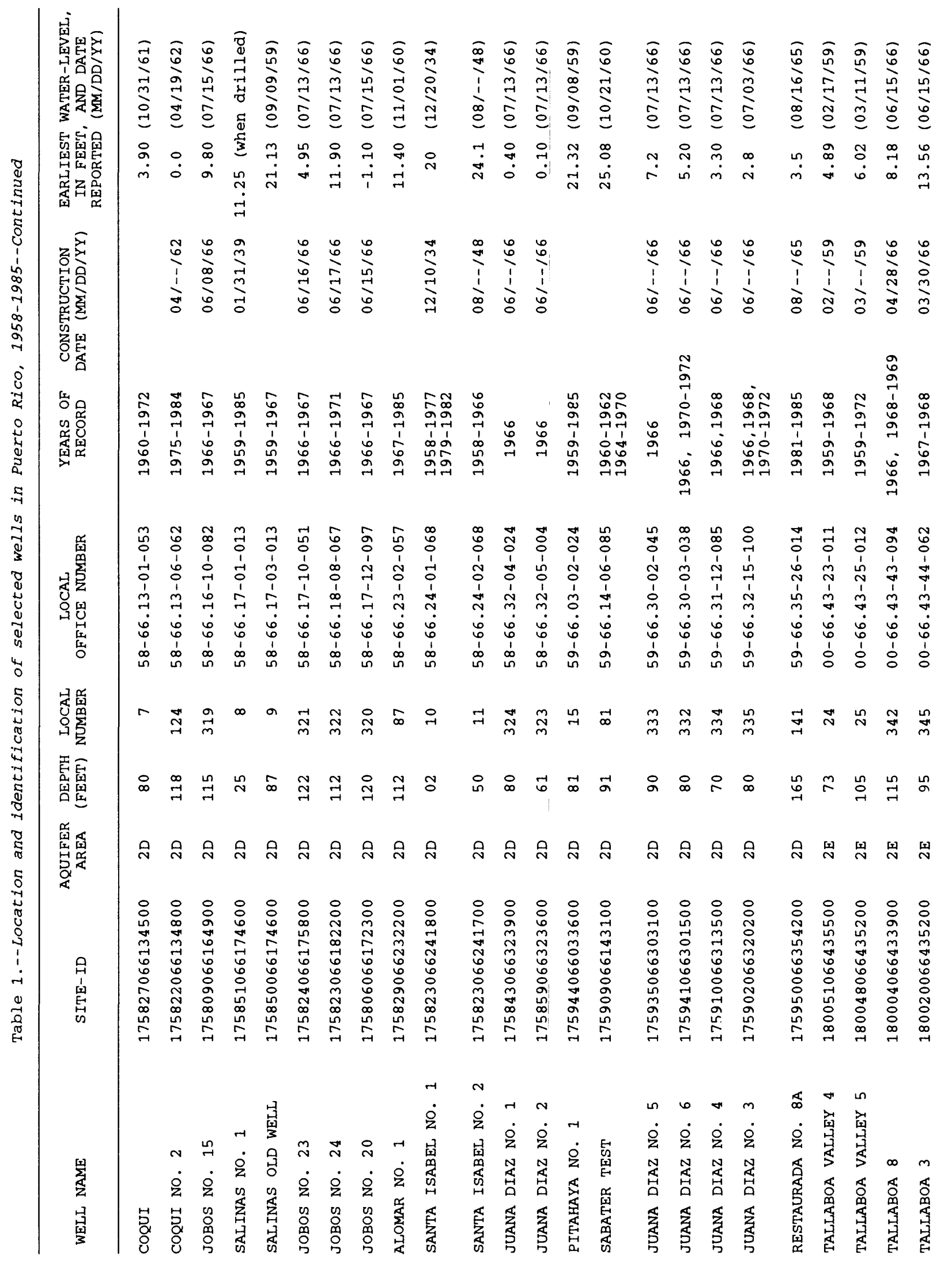




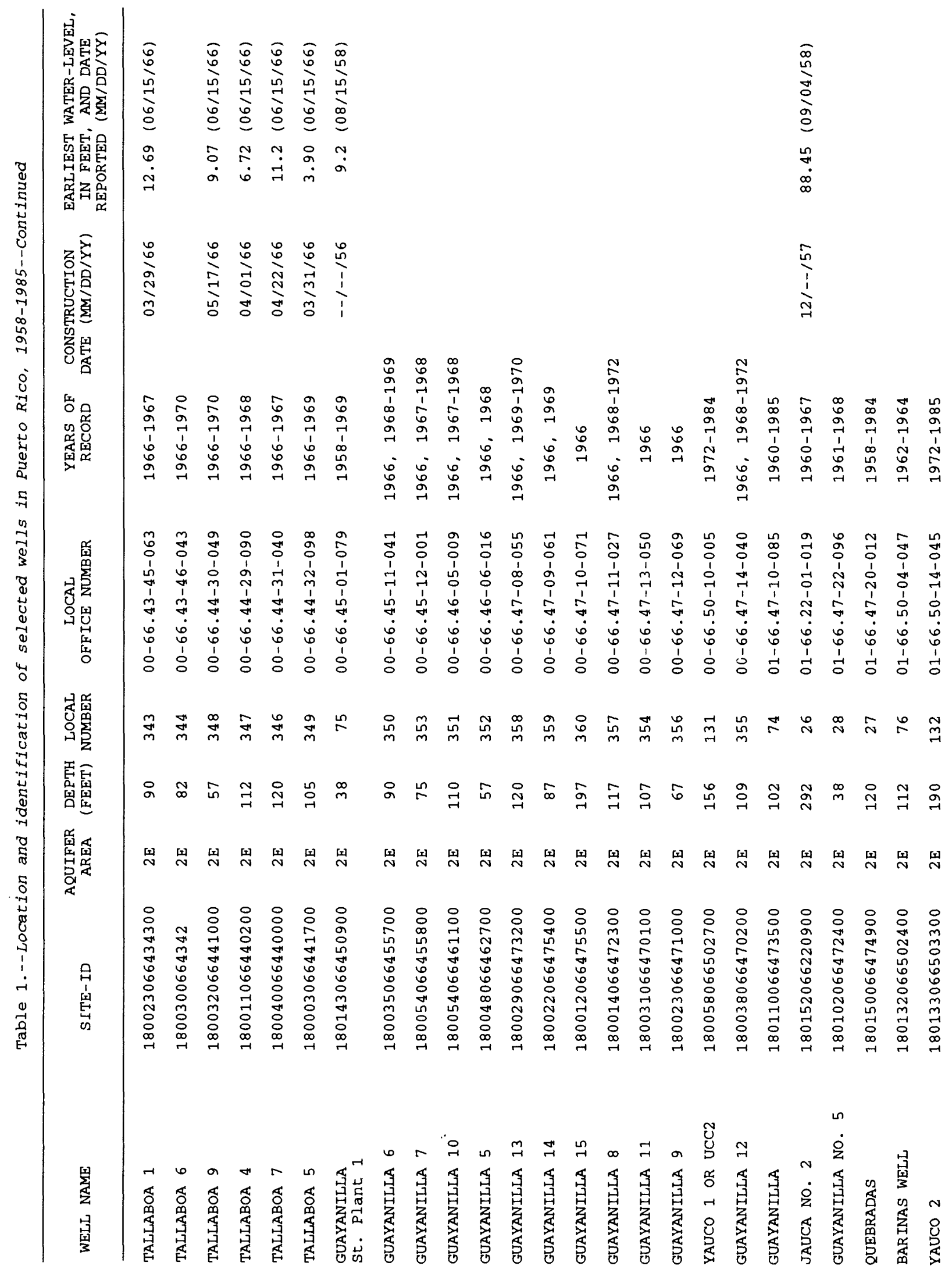




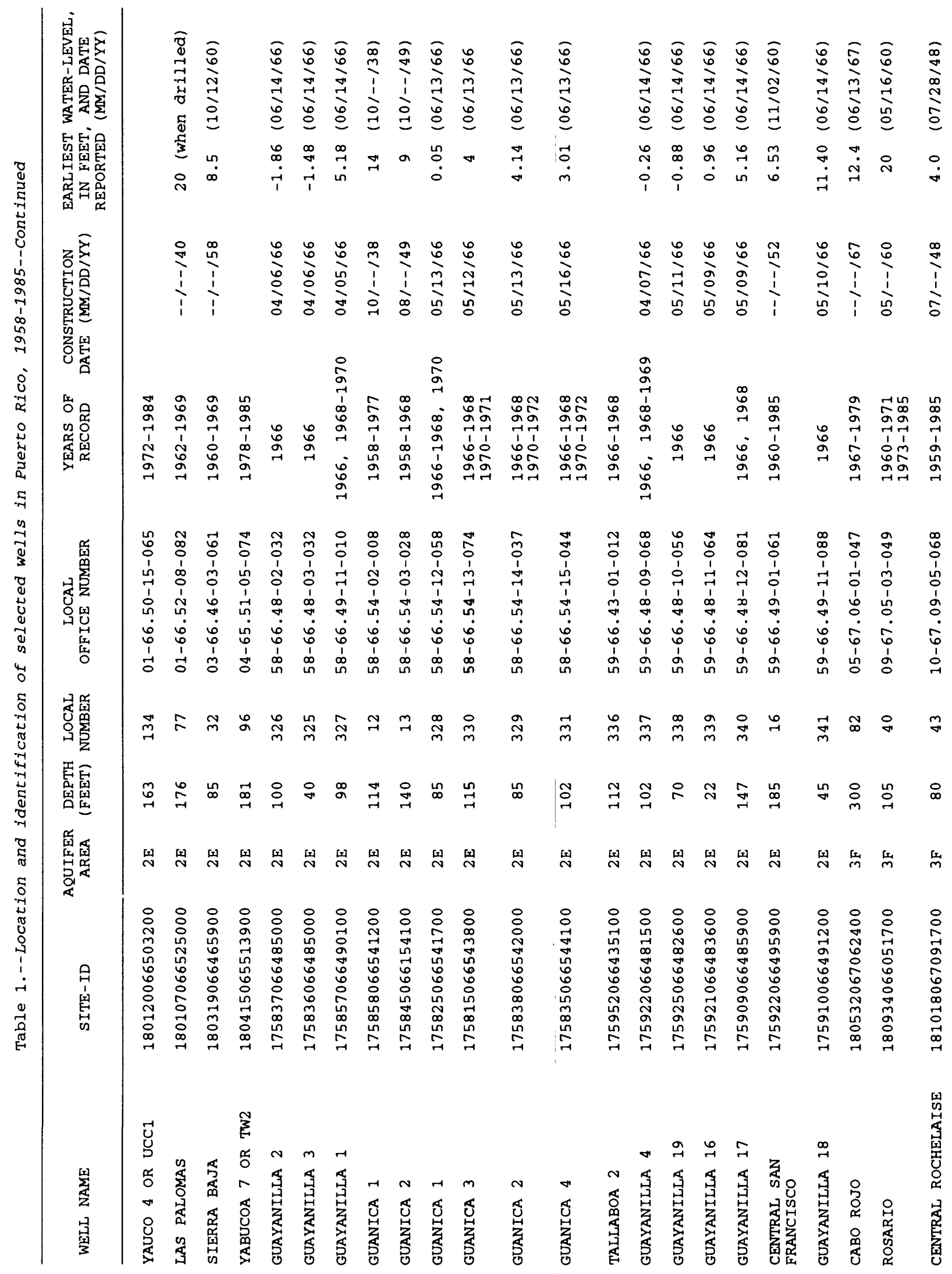




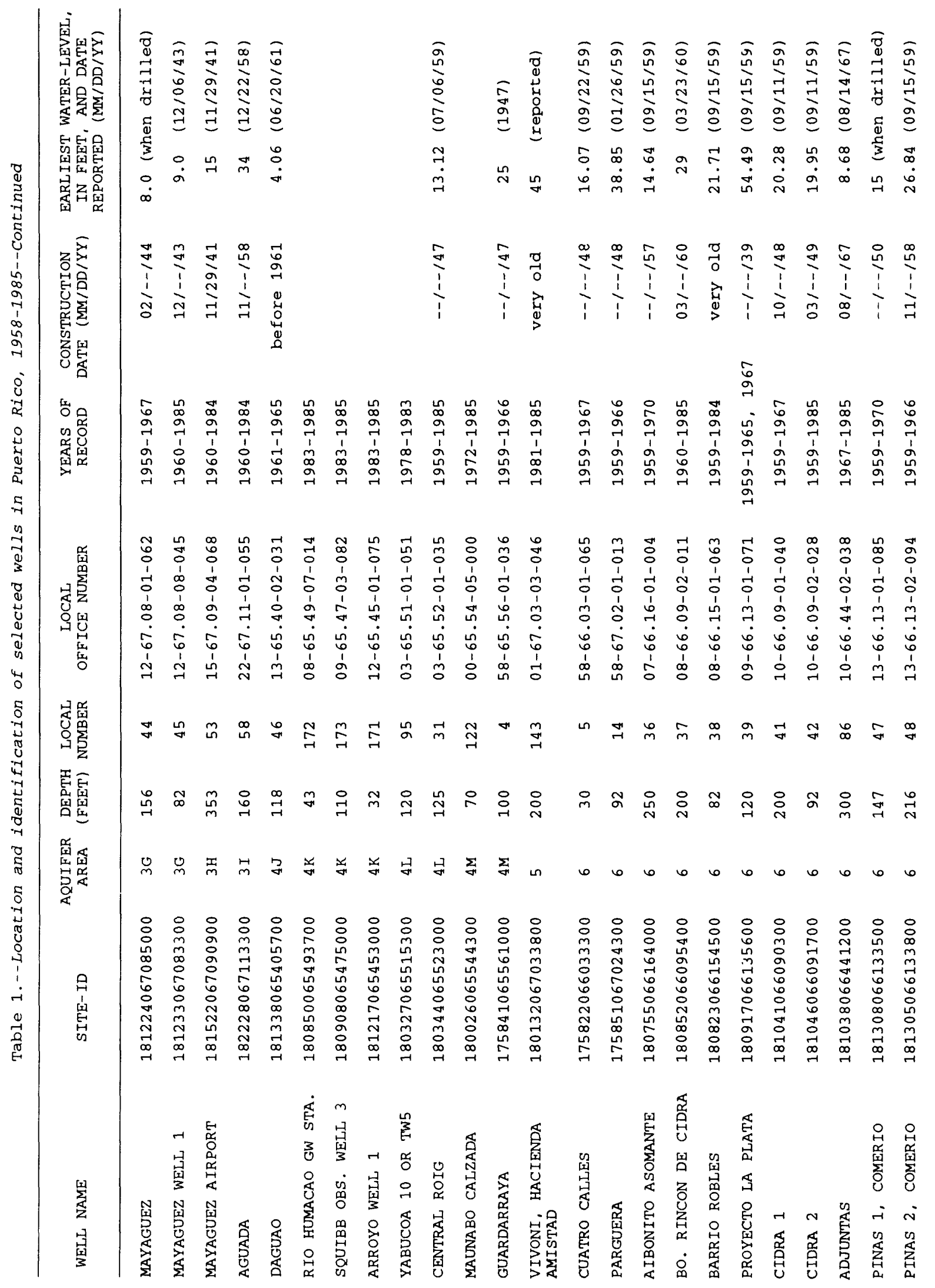




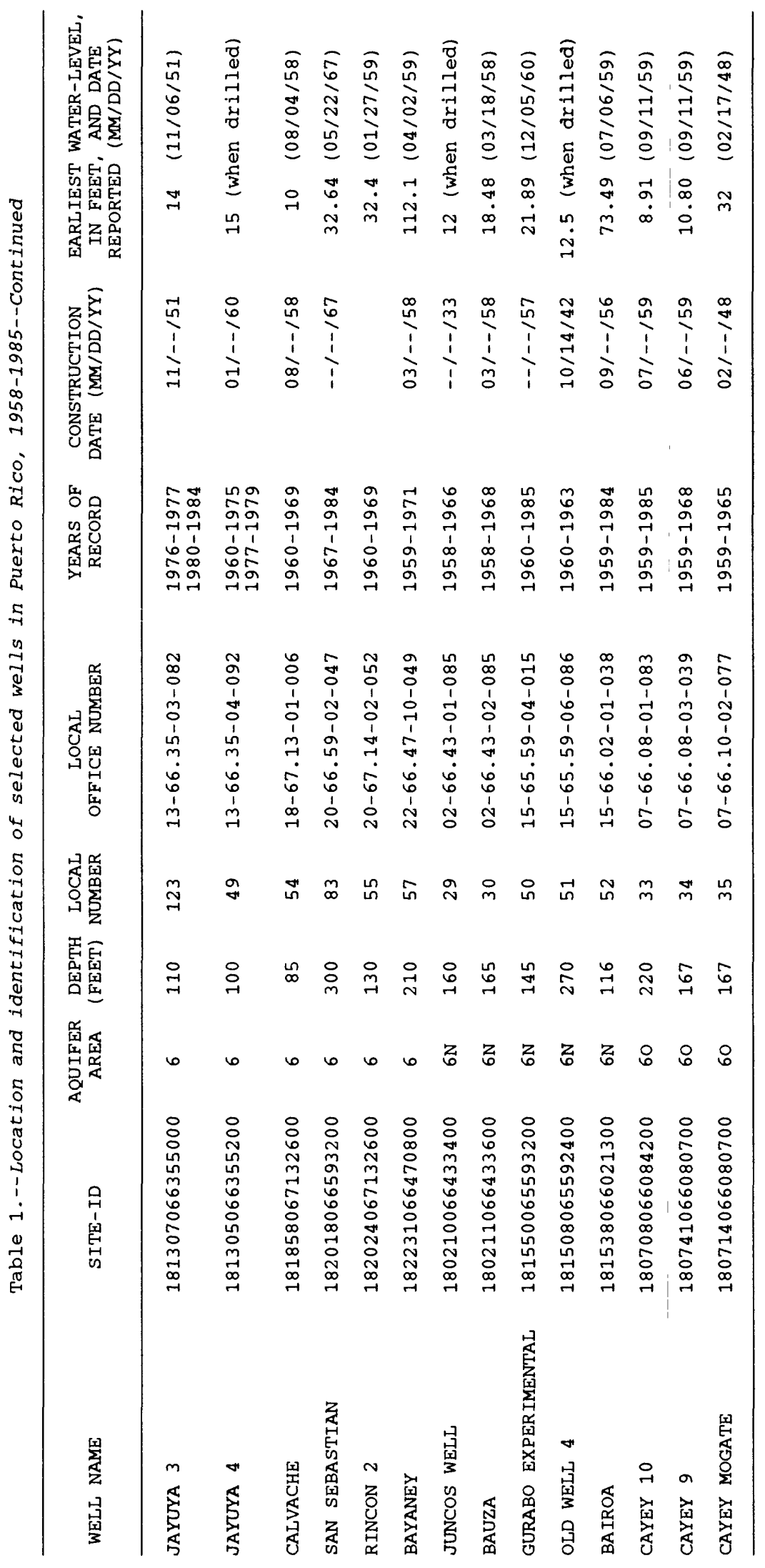




\section{COMPILATION OF GROUND-WATER LEVEL MEASUREMENTS}

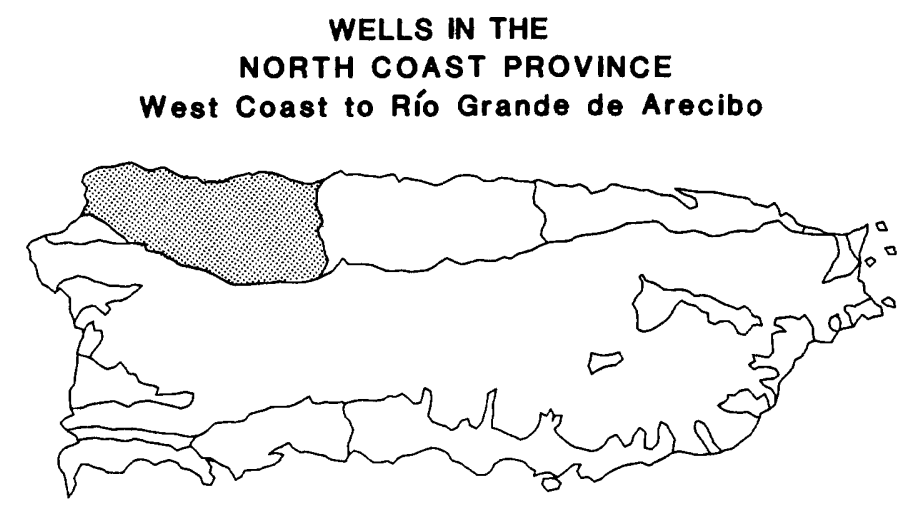

\begin{tabular}{|c|c|c|c|c|c|c|c|c|c|c|c|c|}
\hline $\begin{array}{l}\text { LOCAL } \\
\text { WELL } \\
\text { LAT IT } \\
\text { DIAME }\end{array}$ & $\begin{array}{l}\text { MUMBER : } \\
\text { NAME : Ma } \\
\text { UDE : } 18^{\circ} \\
\text { IER : } 12-\end{array}$ & $\begin{array}{l}165 \\
24^{\prime} \mathrm{P} \text { ' } \\
16 \text { inch }\end{array}$ & Bo. Sal & $\begin{array}{ll} & \text { ELE } \\
\text { cos } & \text { OWN } \\
\text { LON } \\
\text { DEP }\end{array}$ & $\begin{array}{l}\text { VATION } \\
\text { ER : P. } \\
\text { QITUDE } \\
\text { TH : } 20\end{array}$ & $\begin{array}{l}\text { ABOVE } \\
\text { R. Aque } \\
: 67^{\circ} 01 \\
0 \text { feet }\end{array}$ & $\begin{array}{l}\text { AN SEA } \\
\text { duct an } \\
\text { '51" }\end{array}$ & $\begin{array}{l}\text { LEVEL: } \\
\text { Sewer }\end{array}$ & $\begin{array}{l}672.4 \\
\text { Author }\end{array}$ & $\begin{array}{l}\text { feet } \\
\text { ity }\end{array}$ & & \\
\hline .... & : & & & & mo & NTHS & & & & & & \\
\hline YEAR & JAN & FEB & MAR & APR & MAY & JUN & JUL & AUG & SEP & OCT & NOV & $\mathrm{DEC}$ \\
\hline 1982 & : 69.83 & 69.86 & 69.97 & 70.56 & 69.65 & 70.60 & 69.35 & 69.82 & 69.85 & 69.47 & 69.22 & 69.84 \\
\hline 1983 & $: \quad 69.74$ & 69.90 & 69.85 & 70.10 & 69.55 & 69.55 & 69.60 & 69.60 & 69.48 & 69.46 & 69.35 & 66.38 \\
\hline 1984 & $: \quad 69.30$ & 69.29 & 69.27 & 69.22 & 69.30 & 69.13 & 69.42 & 69.18 & 69.08 & 69.08 & 68.99 & 68.88 \\
\hline 1985 & : $\quad 67.89$ & 68.55 & 68.32 & & & & & & & & & \\
\hline
\end{tabular}

LOCAL NUMBER : 164

ELEVATION ABOVE MEAN SEA LEVEL: 787.2 feet

WELL MAME : Rocha MOCa OWMER : P.R. Aqueduct and Sewer Authority

LATITUDE : $18^{\circ} 25^{\prime} 38^{\prime \prime} \quad$ LONGITUDE : 67 $01^{\circ}$ '59"

DIAMETER : 12 inches

DEPTH : 200 feet

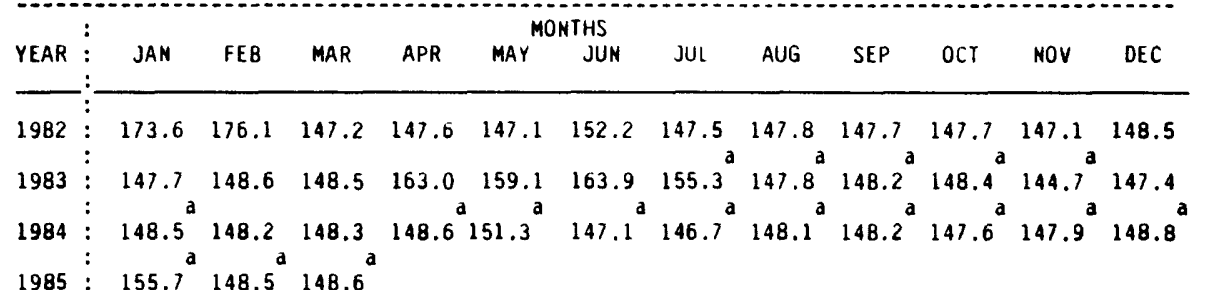

$1985: 155.7148 .5148 .6$

a pumping, b recently pumped, e estimated, $h$ tape measurement, $j$ lowest water level recorded, n nearby punping well 


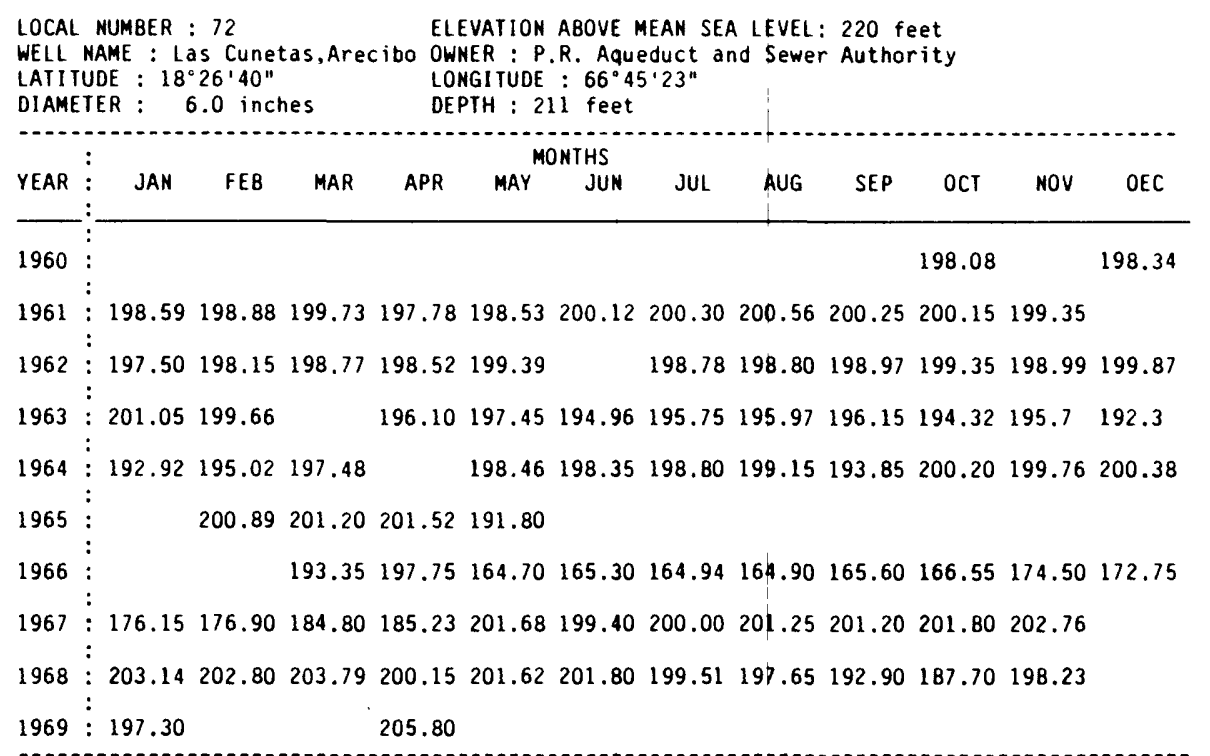

a pumping, b recently pumped, e estimated, $h$ tape measurement, $j$ lowest water level recorded, $n$ nearby puping well

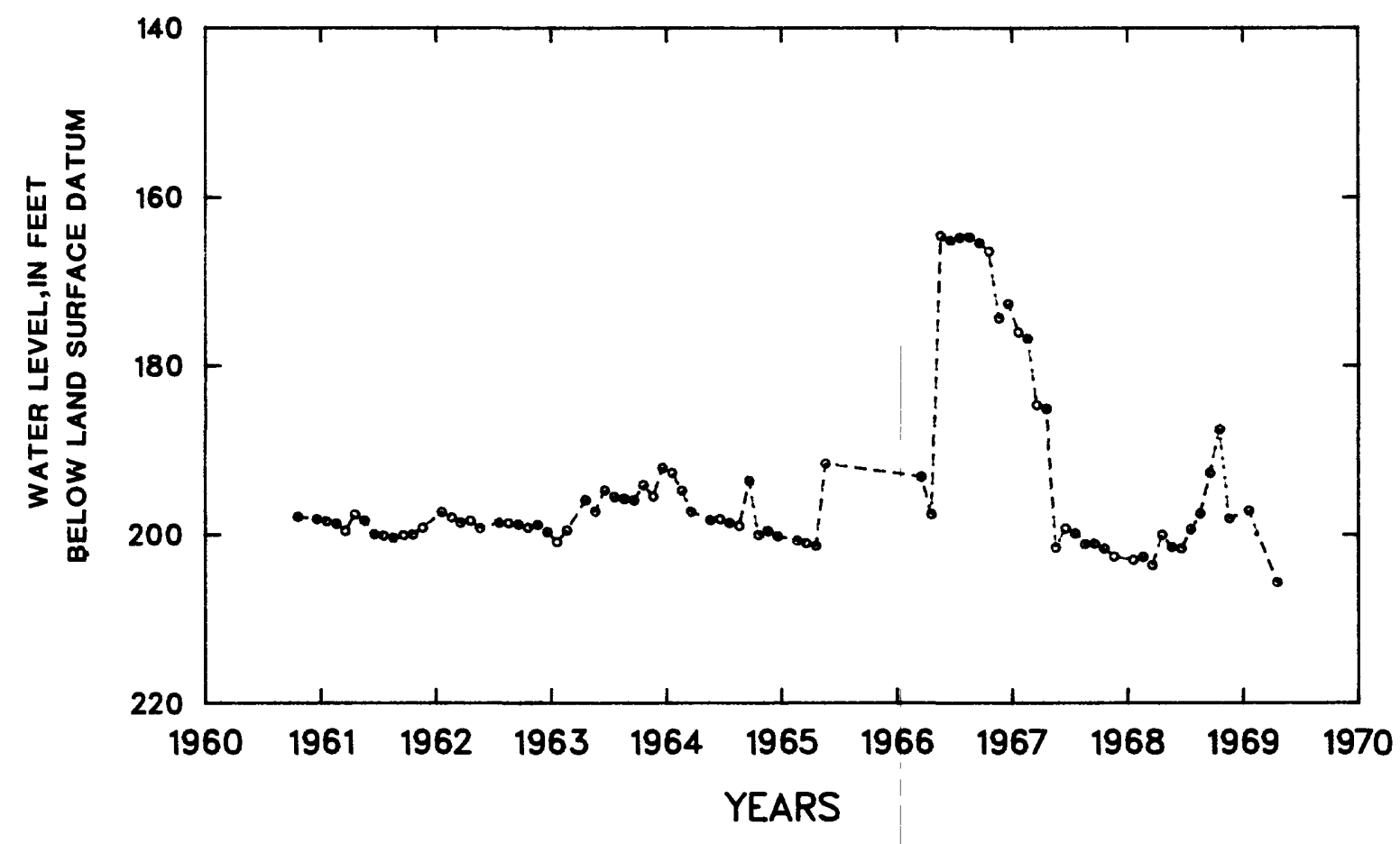

Figure 3.--Ground-water levels at Las Cunetas, Arecibo, local number 72 . 


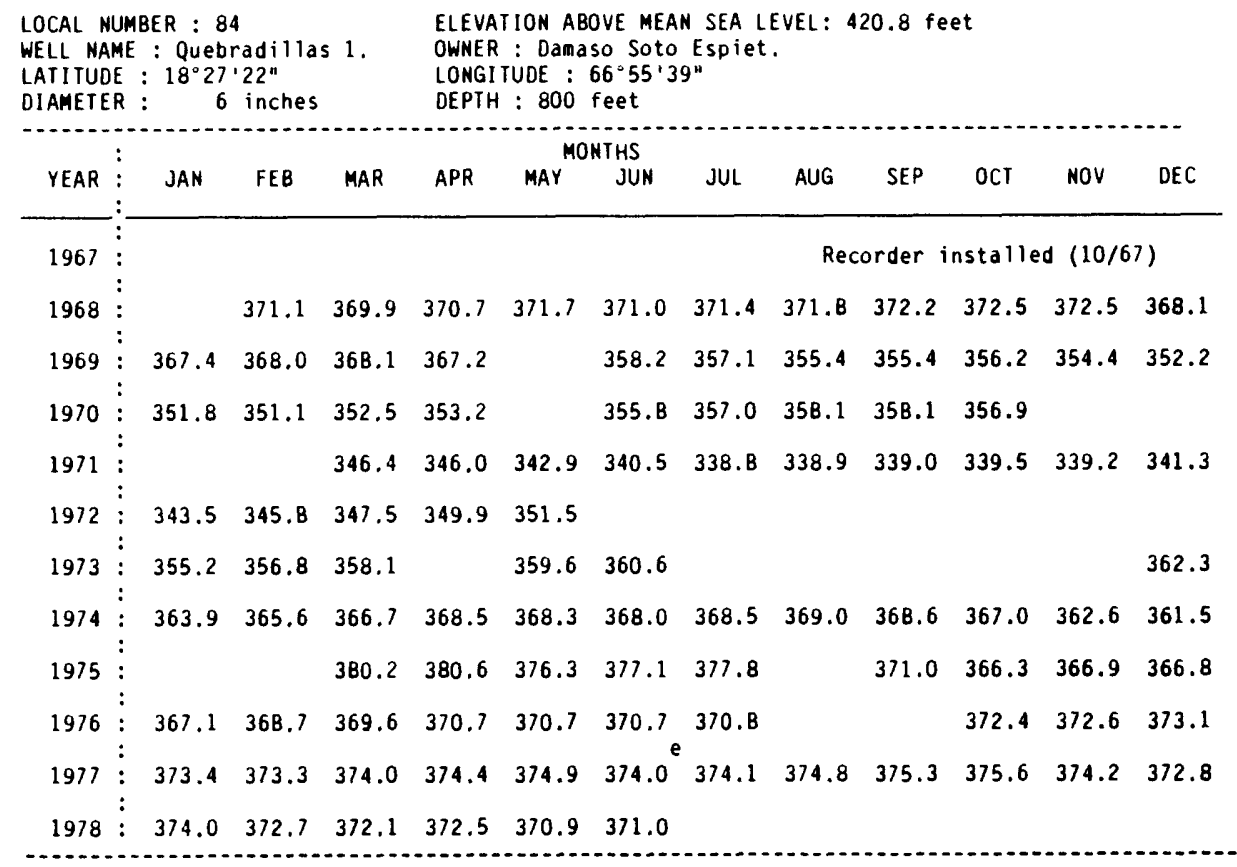

\begin{tabular}{|c|c|c|c|c|c|c|c|c|c|c|c|c|}
\hline $\begin{array}{l}\text { LOCA } \\
\text { WELL } \\
\text { LATI } \\
\text { OIAME }\end{array}$ & $\begin{array}{l}\text { NUMBER } \\
\text { NAME : Q } \\
\text { UDE : } 18 \\
\text { TER : }\end{array}$ & $\begin{array}{l}: 85 \\
\text { Uebradi } \\
\text { '28'58" } \\
6-8 \text { inch }\end{array}$ & $\begin{array}{l}\text { las } 2 \\
\text { ies }\end{array}$ & & $\begin{array}{l}\text { EVATION } \\
\text { NER : S } \\
\text { NGITUDE } \\
\text { PTH : } 2\end{array}$ & $\begin{array}{l}\text { ABOVE } \\
\text { ocial P } \\
: 66^{\circ} 5 \\
50 \text { feet }\end{array}$ & $\begin{array}{l}\text { MEAN SEA } \\
g^{\prime} \text { Adm } \\
.19^{\prime \prime}\end{array}$ & $\begin{array}{l}\text { A LEVEL: } \\
\text {, P.R. }\end{array}$ & $\begin{array}{l}132 \\
\text { Dept }\end{array}$ & $\begin{array}{l}\text { eet } \\
\text { of Agrid }\end{array}$ & Iture & \\
\hline & : & & & & & ONTHS & & & & & & \\
\hline YEAK & JAN & FEB & MAR & APR & MAY & JUN & JUL & $A \cup G$ & SEP & $\mathrm{OCT}$ & NOV & OEC \\
\hline $196 /$ & $\vdots$ & & & & & & & & & 131.57 & 131.56 & 132.01 \\
\hline 1968 & 132.07 & 131.82 & 131.80 & 131.85 & 131.94 & 131.74 & 131.70 & 131.60 & 131.30 & 131.40 & 131.35 & 131.35 \\
\hline 1969 & : 131.25 & 131.30 & 131.30 & 131.50 & 131.40 & 131.40 & 131.30 & & & 131.21 & 131.20 & 130.90 \\
\hline 1970 & $: 130.70$ & & & & & 131.70 & 131.70 & 131.37 & 131.35 & 131.00 & 131.10 & \\
\hline 1971 & : & & 131.20 & 131.50 & & & 130.87 & 131.00 & & & & \\
\hline
\end{tabular}

REMARKS: viscont inued $(09 / 71)$

a pumping, b recently pumped, e estimated, $h$ tape measurement, J lowest water level recorded,

n nearoy pumping will 


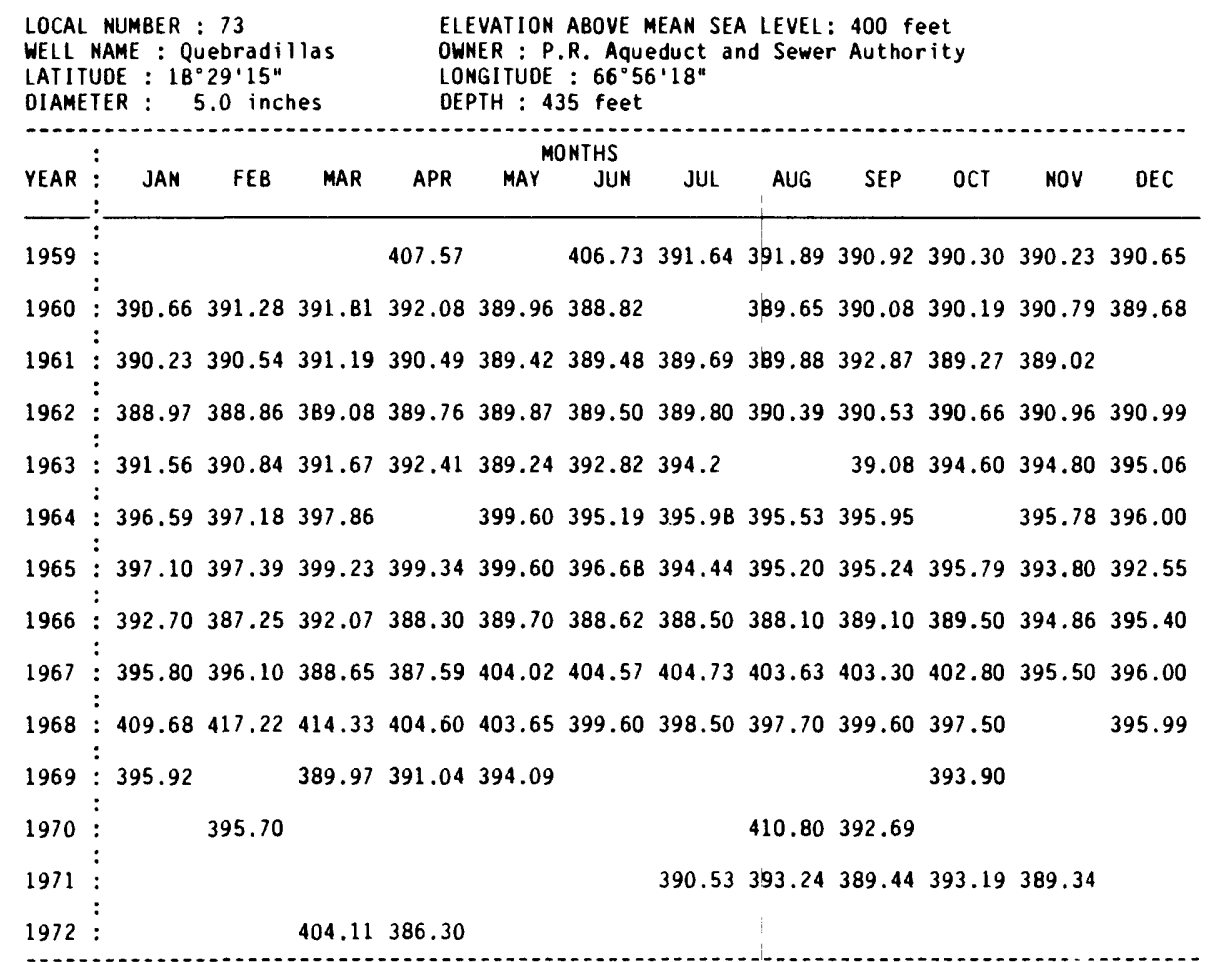

a pumping, b recently pumped, e estimated, $h$ tape measurement, $j$ lowest water level recorded, n nearby pumping well 
THIS PAGE WAS LEFT BLANK INTENTIONALLY 


\section{WELLS IN THE \\ NORTH COAST PROVINCE \\ Río Grande de Arecibo to Río de La Plata}

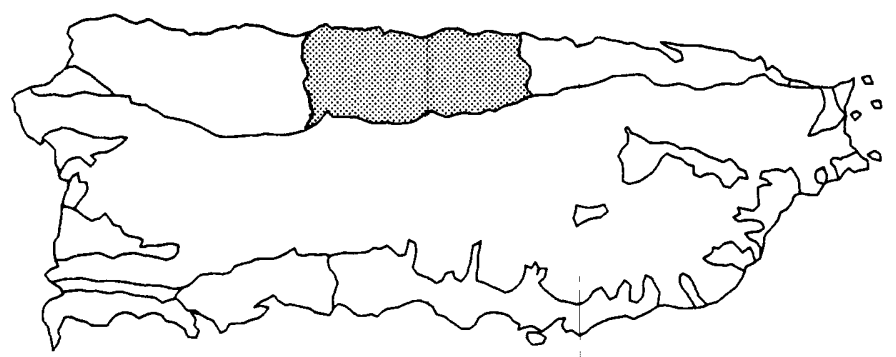

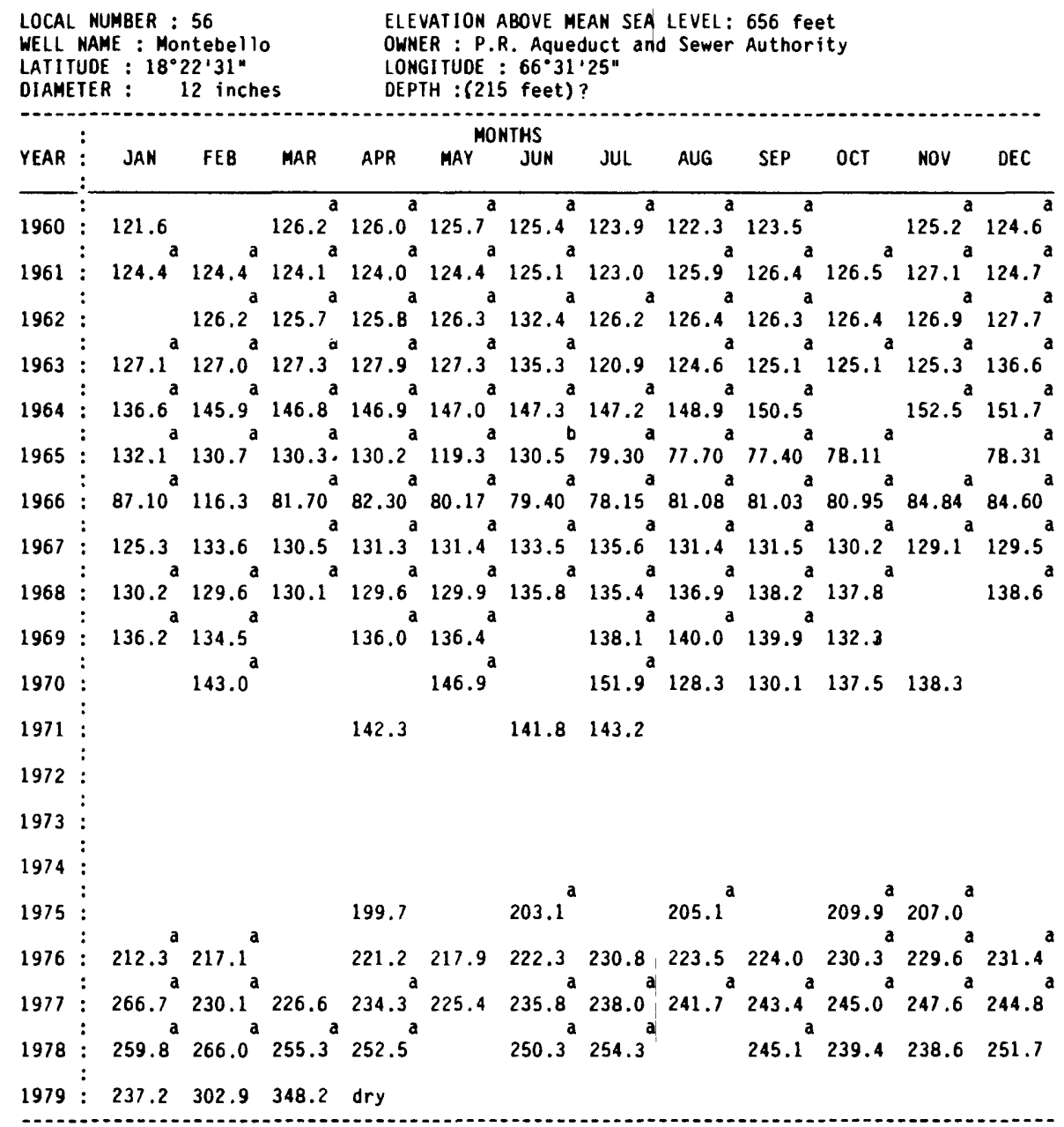

REMARKS: Depth to water measurements do not coincide with the depth of the well.

a pumping, b recently pumped, e estimated, $h$ tape measurement, $j$ lowest water level recorded,

n nearby pumping well 


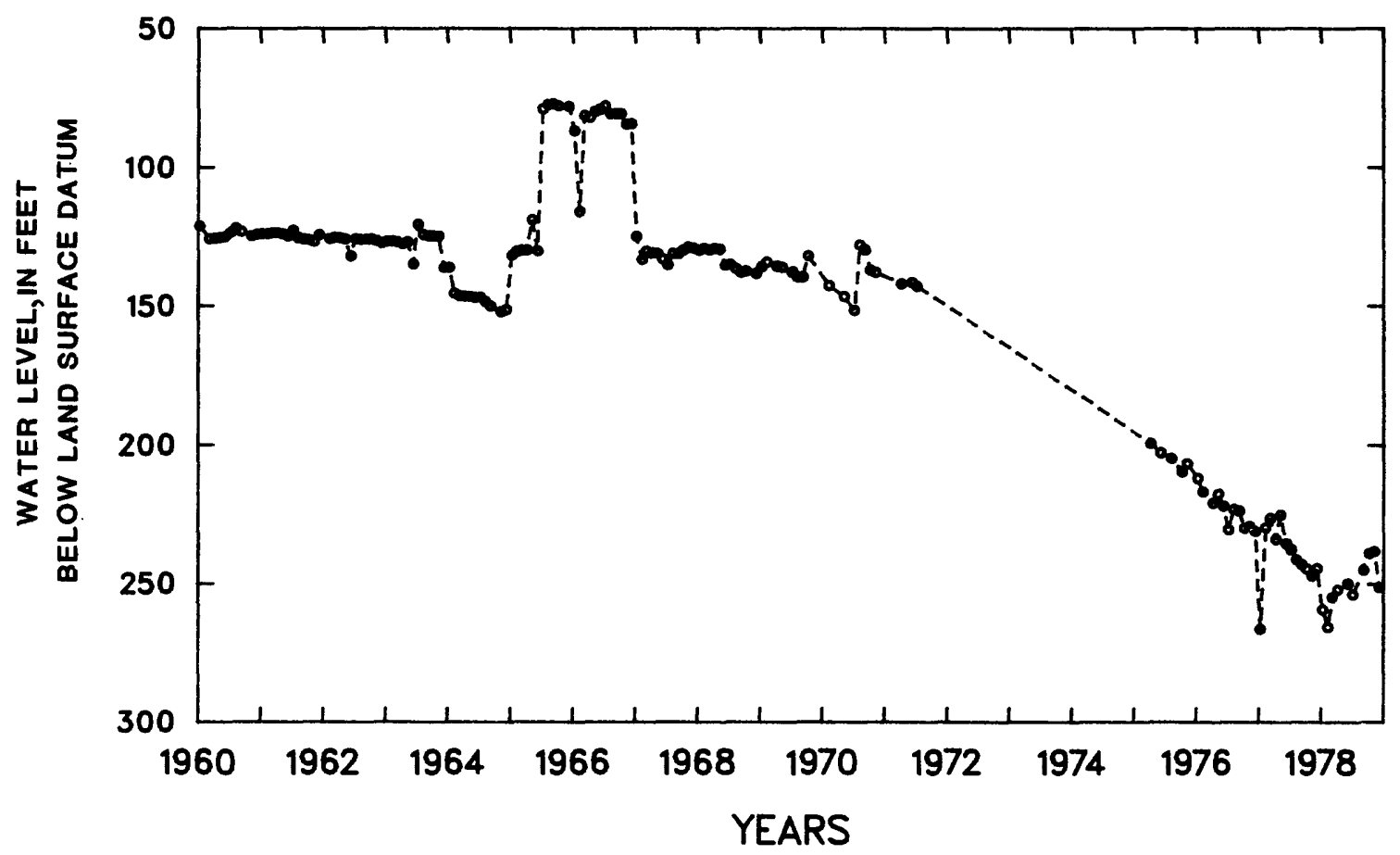

Figure 4.--Ground-water levels at Montebello, local number 56. 


\begin{tabular}{|c|c|c|c|c|c|c|c|c|c|c|c|c|}
\hline \multicolumn{4}{|c|}{$\begin{array}{l}\text { LOCAL NUMBER }: 142 \\
\text { WELL NAME : Morán Simo } \\
\text { LATI TUDE : } 18^{\circ} 24^{\circ} 45^{\prime \prime} \\
\text { DIAMETER : B-20 inches }\end{array}$} & \multicolumn{9}{|c|}{$\begin{array}{l}\text { ELEVATION ABOVE MEAN SEA LEVEL: } 492 \text { feet } \\
\text { OWNER : P.R. Aqueduct and Sewer Authority } \\
\text { LONGITUDE : } 66^{\circ} 31^{\circ} 58^{\prime \prime} \\
\text { DEPTH : } 517^{\prime} \text { feet }\end{array}$} \\
\hline YEAR: & JAN & FEB & MAR & APR & MAY & $\begin{array}{l}\text { NTHS } \\
\text { JUN }\end{array}$ & JUL & AUG & SEP & OCT & NOV & DEC \\
\hline 1981 & $\vdots$ & & & & & & & 22 B. 0 & & & & 228.8 \\
\hline 1982 & : & 227.4 & 22 B. 0 & 227.8 & 226.5 & 227.8 & 228.0 & 228.6 & 228.2 & 228.0 & 228.1 & 228.1 \\
\hline $1983:$ & : 228.1 & 228.2 & 227.2 & 228.4 & 228.6 & 227.8 & 228.3 & 228.5 & 228.6 & 228.5 & 226.6 & 229.0 \\
\hline $1984:$ & : $\quad 228.9$ & 228.8 & 229.1 & 229.3 & 229.9 & 229.9 & 229.0 & 229.0 & 229.1 & 229.1 & 228.9 & 229.0 \\
\hline 1985 & $\vdots 228.8$ & & 229.0 & & & & & & & & & \\
\hline
\end{tabular}

a pumping, $b$ recently pumped, e estimated, $h$ tape measurement, $j$ lowest water level recorded, n nearby pumping well 


\begin{tabular}{|c|c|c|c|c|c|c|c|c|c|c|c|c|}
\hline \multicolumn{4}{|c|}{$\begin{array}{l}\text { LOCAL NUMBER : } 68 \\
\text { WELL NAME : Manatí } 2 \\
\text { LATITUDE : } 18^{\circ} 25^{\prime} 48^{\prime \prime} \\
\text { DIAMETER : } 12-20 \text { inches }\end{array}$} & \multicolumn{9}{|c|}{$\begin{array}{l}\text { ELEVATION ABOVE MEAN SEA LEVEL: } 31.41 \text { feet } \\
\text { OWNER : P.R. Aqueduct and Sewer Authority } \\
\text { LONGITUDE : } 66^{\circ} 30^{\prime} 02^{\prime \prime}\end{array}$} \\
\hline AR & JAN & EFP & MaO & PR & $y^{\prime}$ & 15 & & (6) & & T & Vy & \\
\hline 60 & & & & & & & & & & .51 & 25.98 & .26 \\
\hline 61 & $: \quad 26.46$ & & $27.22^{a}$ & $27.32^{a}$ & 26.12 & $27.30^{\mathrm{a}}$ & $27.10^{a}$ & $26.91^{\mathrm{a}}$ & $26.51^{a}$ & $26.63^{a}$ & $26.66^{a}$ & $25.60^{\mathrm{a}}$ \\
\hline 62 & $\vdots$ & $26.87^{a}$ & $27.11^{a}$ & $27.20^{\mathrm{a}}$ & $26.85^{a}$ & $26.60^{\mathrm{a}}$ & $27.10^{a}$ & $26.90^{\mathrm{a}}$ & $26.79^{a}$ & $26.60^{a}$ & $26.63^{a}$ & $26.74^{\mathrm{a}}$ \\
\hline 33 & $: 26.50^{\mathrm{a}}$ & $26.36^{\mathrm{a}}$ & $26.93^{\mathrm{a}}$ & $25.30^{\mathrm{a}}$ & $25.84^{a}$ & $26.15^{\mathrm{a}}$ & $28.48^{a}$ & $27.68^{a}$ & $27.63^{a}$ & $26.12^{a}$ & $26.05^{a}$ & $24.77^{a}$ \\
\hline 64 & $: 25.40^{\mathrm{a}}$ & & & $27.18^{a}$ & $27.33^{a}$ & $27.55^{\mathrm{a}}$ & $26.80^{\mathrm{a}}$ & $26.98^{a}$ & $26.98^{\mathrm{a}}$ & & $26.95^{\mathrm{a}}$ & 26.60 \\
\hline 65 & $: 27.54^{a}$ & $28.00^{a}$ & $2 \mathrm{~B} .02^{\mathrm{a}}$ & $28.07^{a}$ & $26.12^{a}$ & $22.50^{\mathrm{a}}$ & $25.63^{\mathrm{a}}$ & $26.70^{a}$ & 26.10 & $6.81^{a}$ & $26.97^{\mathrm{a}}$ & 25.59 \\
\hline 6 & $: 23.84^{\mathrm{a}}$ & $25.83^{\mathrm{a}}$ & $26.77^{a}$ & $26.72^{a}$ & $25.50^{a}$ & $26.90^{\mathrm{a}}$ & $28.30^{a}$ & $26.72^{a}$ & $26.82^{a}$ & $27.00^{a}$ & & $8.24^{a}$ \\
\hline 67 & : 26.32 & $28.68^{a}$ & $28.65^{a}$ & $28.59^{\mathrm{a}}$ & $28.73^{a}$ & $29.81^{\mathrm{a}}$ & $28.12^{a}$ & $26.29^{\mathrm{a}}$ & $28.59^{\mathrm{a}}$ & $25.67^{\mathrm{a}}$ & $25.71^{\mathrm{a}}$ & $25.92^{a}$ \\
\hline $6 \mathrm{~B}$ & $: 28.51^{\mathrm{a}}$ & 27.11 & & $29.29^{\mathrm{a}}$ & 27.17 & $28.94^{\mathrm{a}}$ & $28.42^{a}$ & $28.29^{a}$ & $28.50^{\mathrm{a}}$ & $28.38^{a}$ & & \\
\hline 9 & $\begin{array}{l}\vdots \\
\vdots\end{array}$ & 25.89 & & $29.16^{a}$ & $27.50^{a}$ & & $27.47^{a}$ & & $28.01^{a}$ & 26.99 & & \\
\hline 1970 & : & 28.21 & & 28.64 & 25.54 & & 26.23 & 21 & & & & \\
\hline 71 & $: 26.18^{a}$ & & & & $28.62^{a}$ & & $28.64^{a}$ & & & $.89^{\mathrm{a}}$ & $27.26^{a}$ & $27.46^{d}$ \\
\hline 72 & $: 27.25^{\mathrm{a}}$ & & & $27.44^{\mathrm{a}}$ & & 27.83 & $27.90^{\mathrm{a}}$ & $28.00^{a}$ & 25.89 & & & \\
\hline 3 & $: \quad 27.75$ & & 27.87 & 28.37 & & 27.88 & & 26.93 & & $26.89^{a}$ & $27.62^{a}$ & \\
\hline & $: 28.24^{\mathrm{a}}$ & $29.86^{a}$ & & $28.46^{a}$ & $28.56^{a}$ & $26.60^{\circ}$ & $28.01^{a}$ & & $28.09^{\mathrm{a}}$ & & $24.05^{a}$ & $23.18^{\circ}$ \\
\hline 1975 & $: 37.19^{a}$ & & 28.12 & $28.44^{a}$ & & $28.93^{\mathrm{a}}$ & & $28.76^{a}$ & & $28.37^{a}$ & 28.62 & \\
\hline 76 & & 28.60 & & .04 & 25.96 & 0 & 29.96 & & 8 & $9.95^{a}$ & $29.49^{a}$ & $31.14^{a}$ \\
\hline 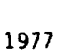 & $30.22^{a}$ & $30.21^{a}$ & $31.83^{a}$ & $29.83^{a}$ & $31.83^{\mathrm{a}}$ & 28.71 & $29.75^{\mathrm{a}}$ & $32.70^{a}$ & $32.57^{\mathrm{a}}$ & $32.80^{a}$ & $32.34^{\mathrm{a}}$ & 28.10 \\
\hline 8 & $28.85^{\mathrm{a}}$ & $32.87^{\mathrm{a}}$ & $28.75^{\mathrm{a}}$ & $27.23^{a}$ & & $30.80^{\mathrm{a}}$ & $32.60^{a}$ & $32.55^{\mathrm{a}}$ & $32.94^{\mathrm{a}}$ & $32.07^{\circ}$ & $32.62^{\mathrm{a}}$ & 33.05 \\
\hline 1979 & $30.00^{\mathrm{a}}$ & $35.02^{a}$ & $34.40^{\circ}$ & $28.84^{a}$ & $28.77^{a}$ & $27.99^{\mathrm{a}}$ & $29.83^{a}$ & $28.75^{\mathrm{a}}$ & $26.33^{\mathrm{a}}$ & $27.12^{a}$ & $26.22^{\mathrm{a}}$ & $26.20^{\mathrm{a}}$ \\
\hline 1980 & $27.97^{a}$ & & $28.46^{a}$ & $28.66^{a}$ & $29.08^{a}$ & $28.51^{\circ}$ & $29.17^{a}$ & $29.26^{a}$ & $29.29^{a}$ & $29.38^{a}$ & $29.50^{\mathrm{a}}$ & $28.82^{a}$ \\
\hline 31 & $29.09^{a}$ & $30.18^{\circ}$ & & & & 25.08 & $28.50^{a}$ & $25.93^{\mathrm{a}}$ & & $29.19^{a}$ & $25.13^{\mathrm{a}}$ & $29.12^{2}$ \\
\hline 1982 & $25.69^{\mathrm{a}}$ & $28.30^{\mathrm{a}}$ & $29.00^{a}$ & $29.58^{\mathrm{a}}$ & $29.60^{a}$ & $29.40^{\circ}$ & $29.62^{a}$ & $29.31^{a}$ & $29.28^{a}$ & $28.92^{a}$ & $29.09^{a}$ & $29.19^{a}$ \\
\hline 1983 & $28.68^{a}$ & $29.36^{a}$ & $29.38^{a}$ & $29.80^{\mathrm{a}}$ & $26.40^{a}$ & $29.04^{\circ}$ & $29.10^{a}$ & $29.30^{\mathrm{a}}$ & $29.38^{\mathrm{a}}$ & $29.14^{a}$ & $29.05^{\mathrm{a}}$ & 29.24 \\
\hline 1984 & $29.50^{a}$ & $29.26^{a}$ & $29.39^{\mathrm{a}}$ & $29.77^{\mathrm{a}}$ & $29.19^{\circ}$ & $28.66^{\mathrm{a}}$ & $29.43^{a}$ & $29.27^{\mathrm{a}}$ & $29.28^{a}$ & $26.80^{a}$ & $28.77^{\mathrm{a}}$ & $28.85^{a}$ \\
\hline
\end{tabular}

REMARKS: Well discontinued.

a pumping, b recently pumped, e estimated, $h$ tape measurement, $j$ lowest water level recorded, n nearby pumping well 


\begin{tabular}{|c|c|c|c|c|c|c|c|c|c|c|c|c|}
\hline $\begin{array}{l}\text { LOCAL } \\
\text { WELL } \\
\text { LATI } \\
\text { DIAME }\end{array}$ & $\begin{array}{l}\text { NUMBER : } \\
\text { NAME : Ma } \\
\text { UDE : } 18^{\circ} \\
\text { TER : } 20-\end{array}$ & $\begin{array}{l}166 \\
\text { atí Ne } \\
25^{\prime} 42^{\prime \prime} \\
14 \text { inch }\end{array}$ & Well & $\begin{array}{l}\text { ELE } \\
\text { OWN } \\
\text { LON } \\
\text { DEP }\end{array}$ & $\begin{array}{l}\text { VATION } \\
\text { ER : P. } \\
\text { GITUDE } \\
\text { TH : } 14\end{array}$ & $\begin{array}{l}\text { ABOVE } \\
\text { २. Aque } \\
66^{\circ} 30 \\
\text { feet }\end{array}$ & $\begin{array}{l}\text { EAN SEA } \\
\text { duct an } \\
52^{\prime \prime}\end{array}$ & $\begin{array}{l}\text { EVEL: } \\
\text { Sewer }\end{array}$ & $\begin{array}{l}29.52 \\
\text { Autho }\end{array}$ & $\begin{array}{l}\text { eet } \\
\text { ty }\end{array}$ & & \\
\hline & : & & & & MO & NTHS & & & & & & \\
\hline YEAR & JAN & FEB & MAR & APR & MAY & JUN & JUL & AUG & SEP & ОСТ & NOV & DEC \\
\hline 1982 & $: 24.35$ & 23.57 & 24.96 & 25.16 & 24.53 & 25.04 & 25.20 & 25.37 & 25.27 & 24.99 & 25.10 & 25.05 \\
\hline 1983 & $: \quad 24.88$ & 26.36 & 22.90 & 25.45 & 24.85 & 25.16 & 25.20 & 25.55 & 25.47 & 25.34 & 25.17 & 25.43 \\
\hline 1984 & $: 26.05$ & 25.55 & 25.78 & 25.55 & 25.41 & 25.53 & 25.98 & 25.96 & 25.85 & 25.46 & 24.85 & 25.31 \\
\hline
\end{tabular}

REMARKS: Well discont inued.

a pumping, b recently pumped, e estimated, $h$ tape measurement, $j$ lowest water level recorded,

n nearby pumping well 
THIS PAGE WAS LEFT BLANK INTENTIONALLY 


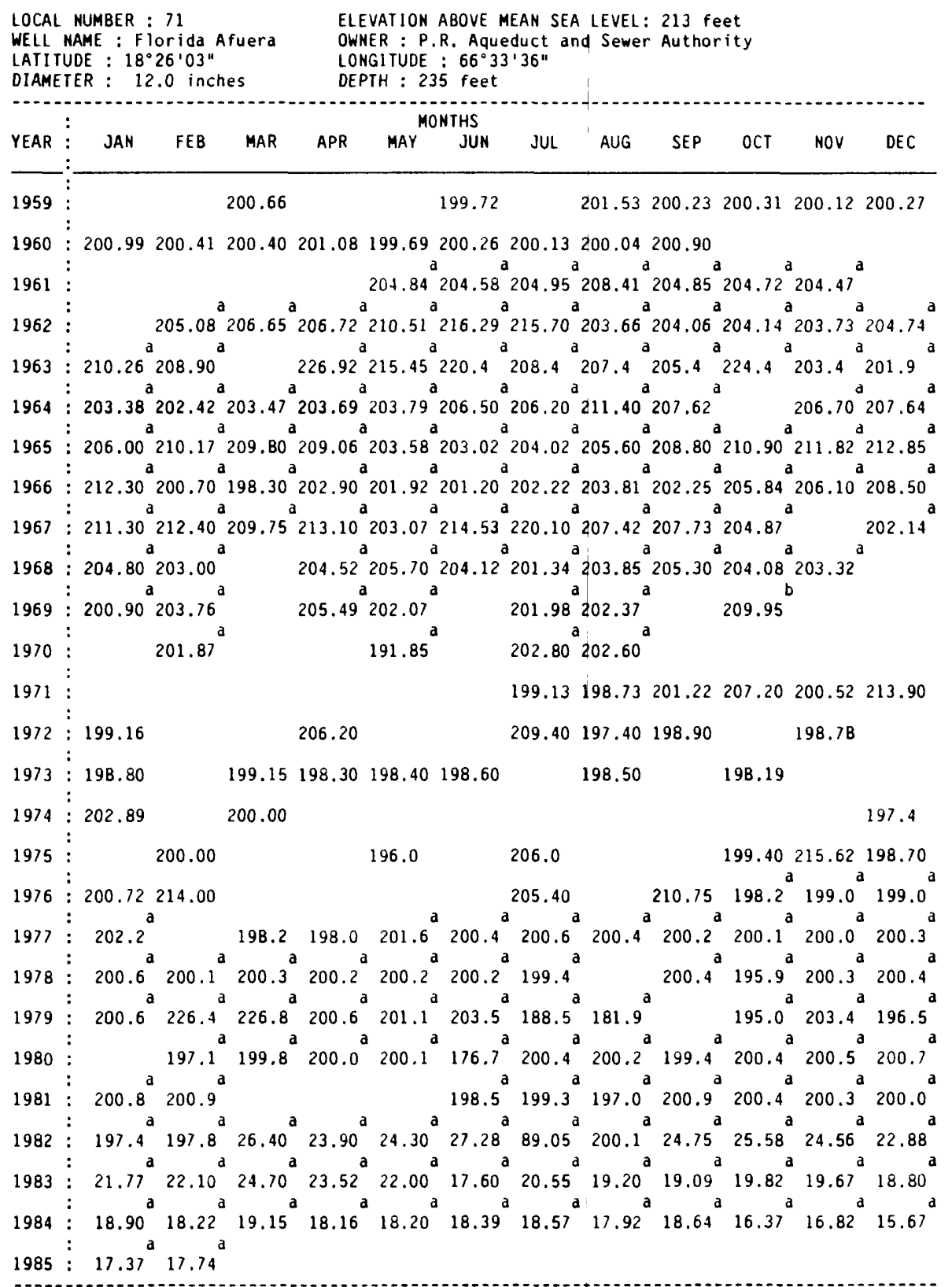

REMARKS: Abrupt change in depth to water corroborated with field inspector.

a pumping, b recently pumped, estimated, $h$ tape measurement, $j$ lowest water level recorded. n nearby pumping well 


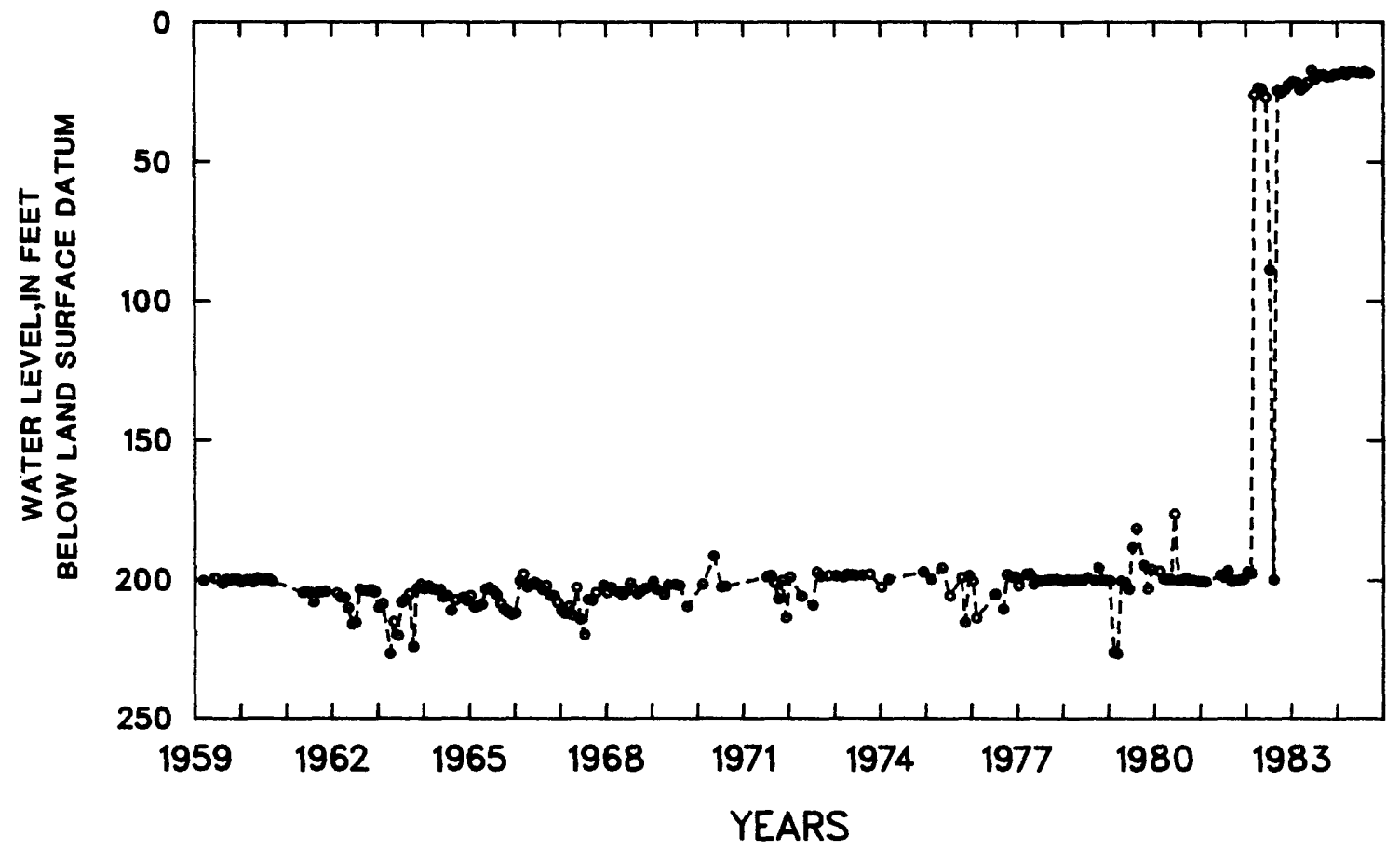

Figure 5.--Ground-water levels at Florida Afuera, local number 71. 


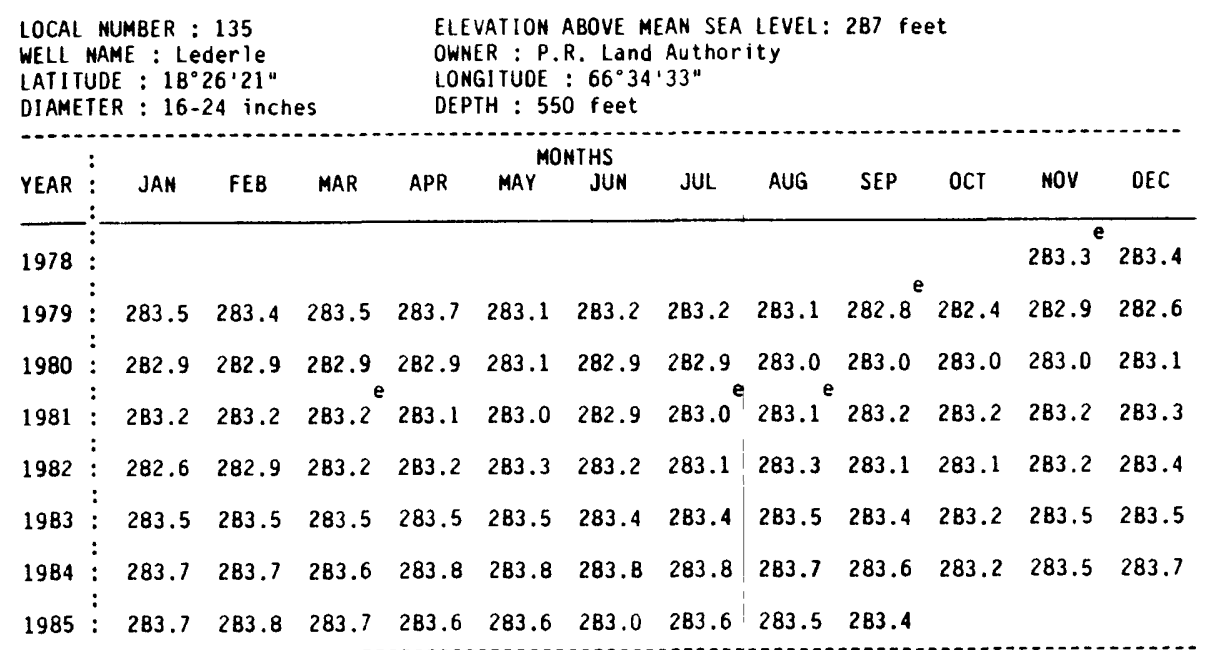

\begin{tabular}{|c|c|c|c|c|c|c|c|c|c|c|c|c|}
\hline $\begin{array}{l}\text { LOCAL } \\
\text { WELL } \\
\text { LATIT } \\
\text { DIAME }\end{array}$ & $\begin{array}{l}\text { LUMBER : } \\
\text { NAME : Sa } \\
\text { TUDE : } 18^{\circ} \\
\text { ETER : } 10-\end{array}$ & $\begin{array}{l}161 \\
\text { tana No } \\
6.32 " \\
2 \text { inch }\end{array}$ & & $\begin{array}{l}\text { ELEI } \\
\text { OWN } \\
\text { LONC } \\
\text { DEP }\end{array}$ & $\begin{array}{l}\text { VATION } \\
\text { ER : P. } \\
\text { GITUDE } \\
\text { TH : } 22\end{array}$ & $\begin{array}{l}\text { BOVE M } \\
\text { Aque } \\
66^{\circ} 38 \\
\text { feet }\end{array}$ & $\begin{array}{l}\text { EAN SEA } \\
\text { duct an } \\
\text { ' } 4 B^{\prime \prime}\end{array}$ & $\begin{array}{l}\text { EVEL: } \\
\text { Sewer }\end{array}$ & $\begin{array}{l}14 \mathrm{~B} \text { fe } \\
\text { Author }\end{array}$ & & & \\
\hline YEAR & JAN & FEB & MAR & APR & - MAY $^{\text {MO }}$ & $\begin{array}{l}\text { ITHS } \\
\text { JUN }\end{array}$ & JUL & AUG & SEP & $\mathrm{OCT}$ & MOV & $\mathrm{DEC}$ \\
\hline 1982 & $: 113.7^{\mathrm{a}}$ & $130.1^{a}$ & $122.7^{a}$ & $139.7^{a}$ & $134.9^{a}$ & $130.5^{a}$ & $142.8^{a}$ & $136.8^{a}$ & $140.2^{a}$ & $146.6^{a}$ & $135.9^{a}$ & $12 B .4^{\mathrm{a}}$ \\
\hline 1983 & $: \quad 129.6^{\mathrm{a}}$ & $132.0^{\mathrm{a}}$ & $12 B \cdot 3^{a}$ & 117.4 & $152.2^{a}$ & & $151.3^{a}$ & $139 . \mathrm{B}$ & $148.4^{a}$ & $138.4^{a}$ & $132.1^{a}$ & $133.0^{a}$ \\
\hline 1984 & ${ }^{:} \quad 132 . \mathrm{B}^{\mathrm{a}}$ & $132.2^{\mathbf{a}}$ & $133.1^{a}$ & $142.9^{a}$ & $132.0^{\mathrm{a}}$ & $125.8^{a}$ & $134.2^{a}$ & $129.2^{a}$ & $132.5^{a}$ & $132.4^{a}$ & & $132.5^{\mathrm{a}}$ \\
\hline
\end{tabular}

REMARKS: Well discont inued.

a pumping, b recently pumped, e estimated, $h$ tape measurement, $j$ lowest water level recorded, n nearby pumping well 
WELLS IN THE

NORTH COAST PROVINCE

Río de La Plata to Río Espíritu Santo

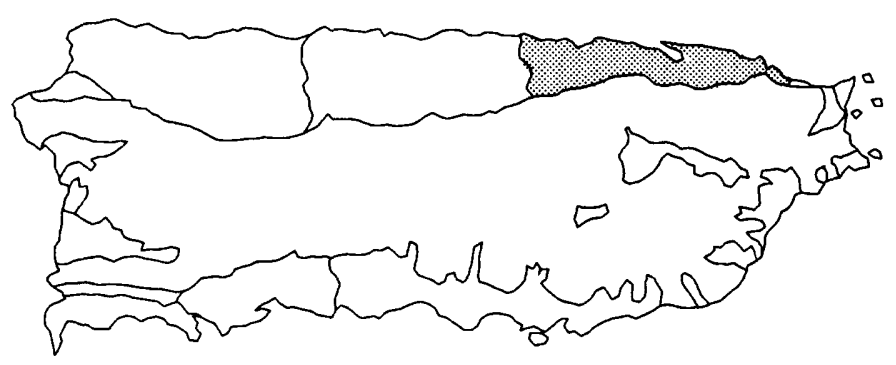

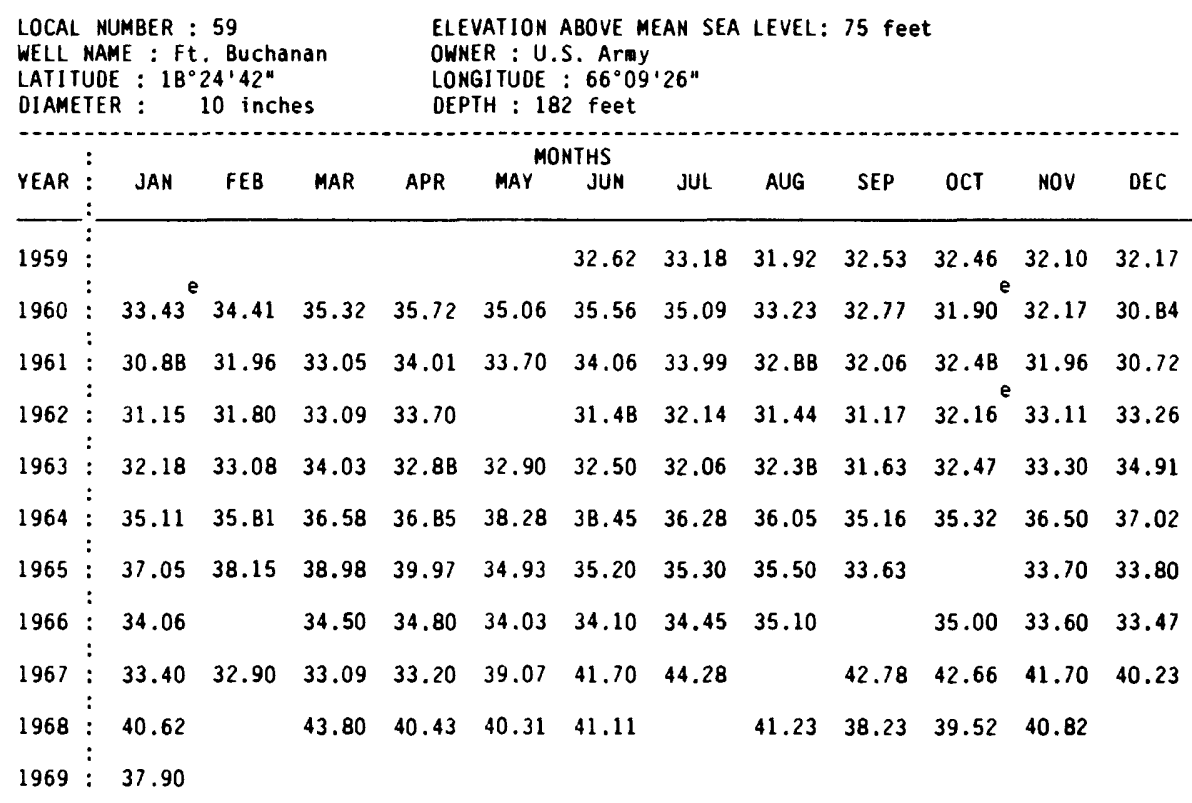

REMARKS: Well discont inued August, 1969.

a pumping, b recently pumped, e estimated, $h$ tape measurement, $j$ lowest water level recorded,

$n$ nearby pumping well 


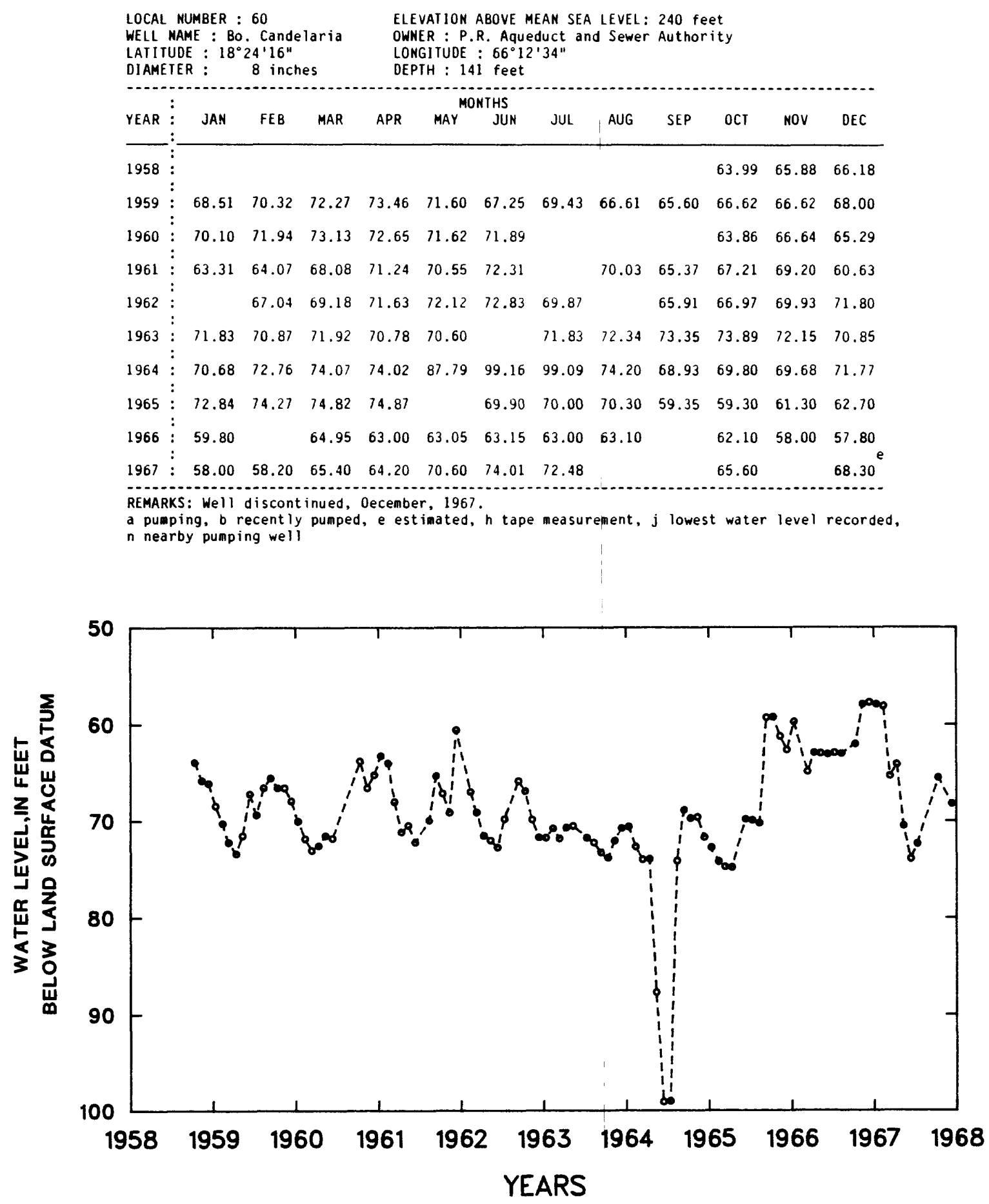

Figure 6.--Ground-water levels at Barrio Candelaria, local number 60. 


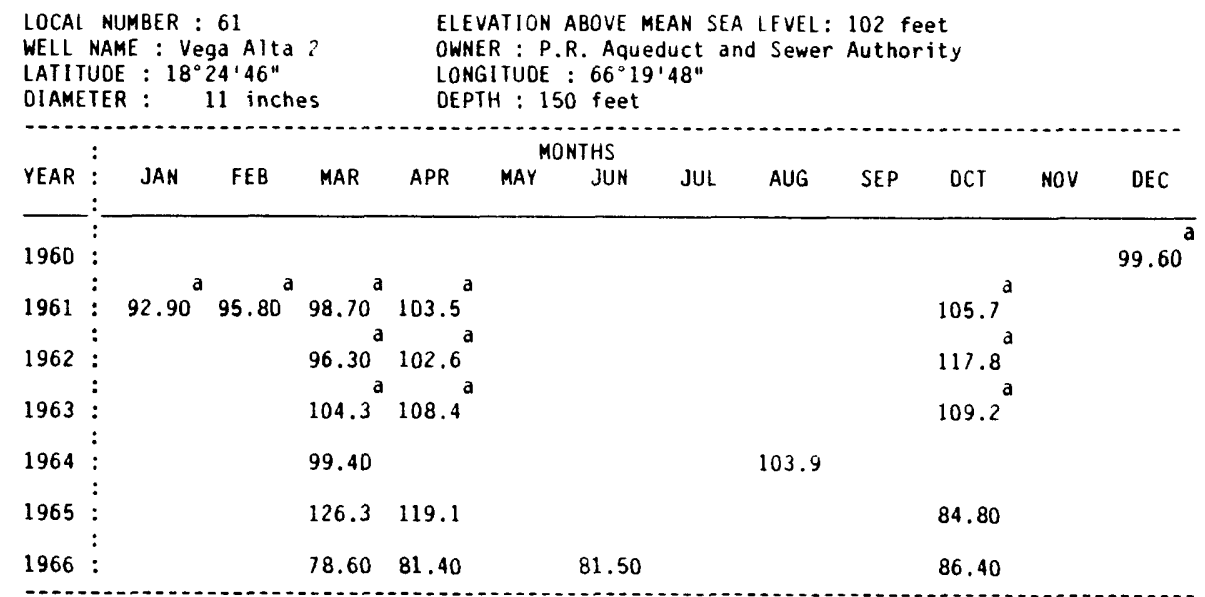

REMARKS: Well discontinued.

a pumping, b recently pumped, e estimated, h tape measurement, $j$ lowest water level recorded, n nearby pumping well

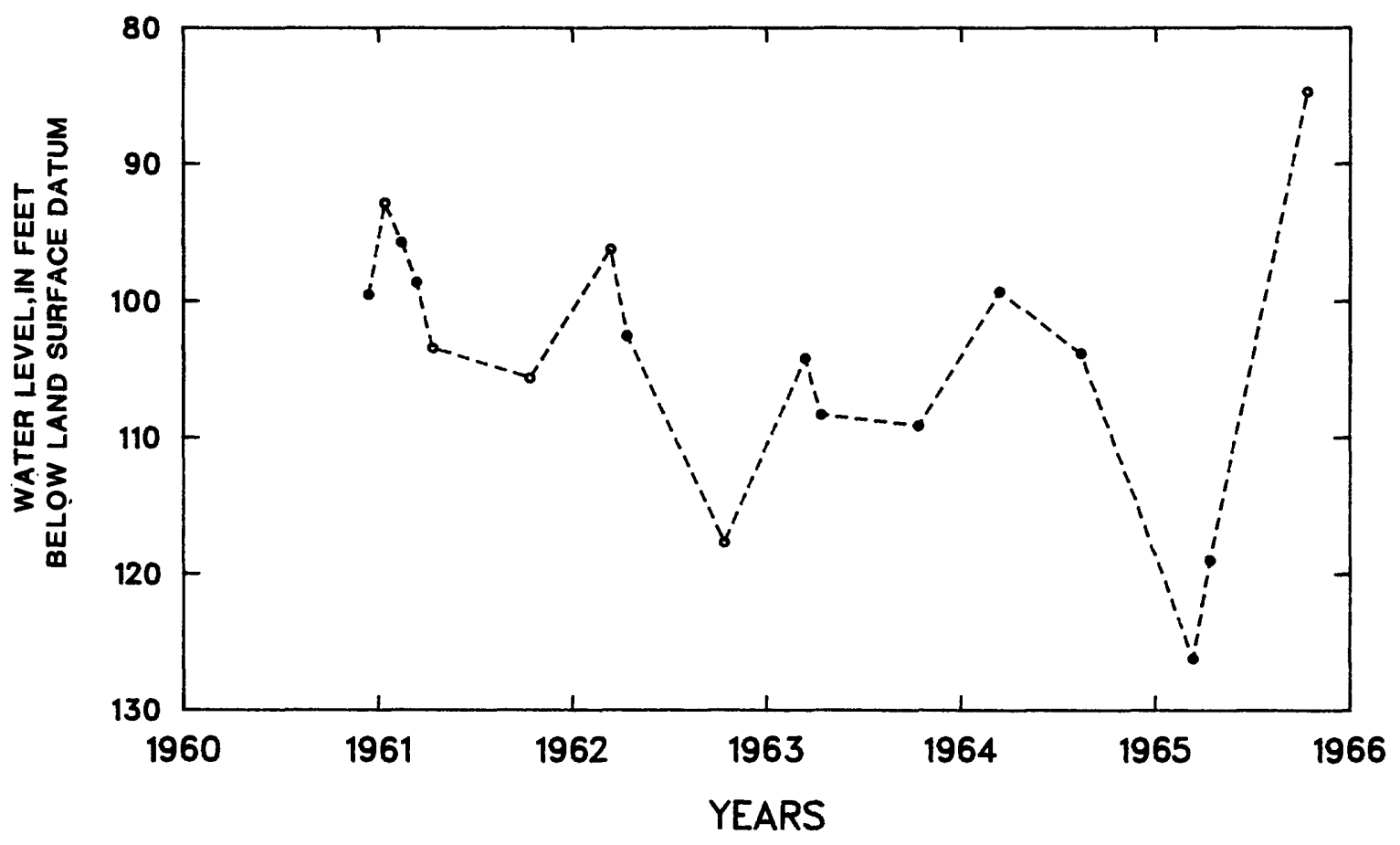

Figure 7.--Ground-water levels at Vega Alta 2, local number 61. 


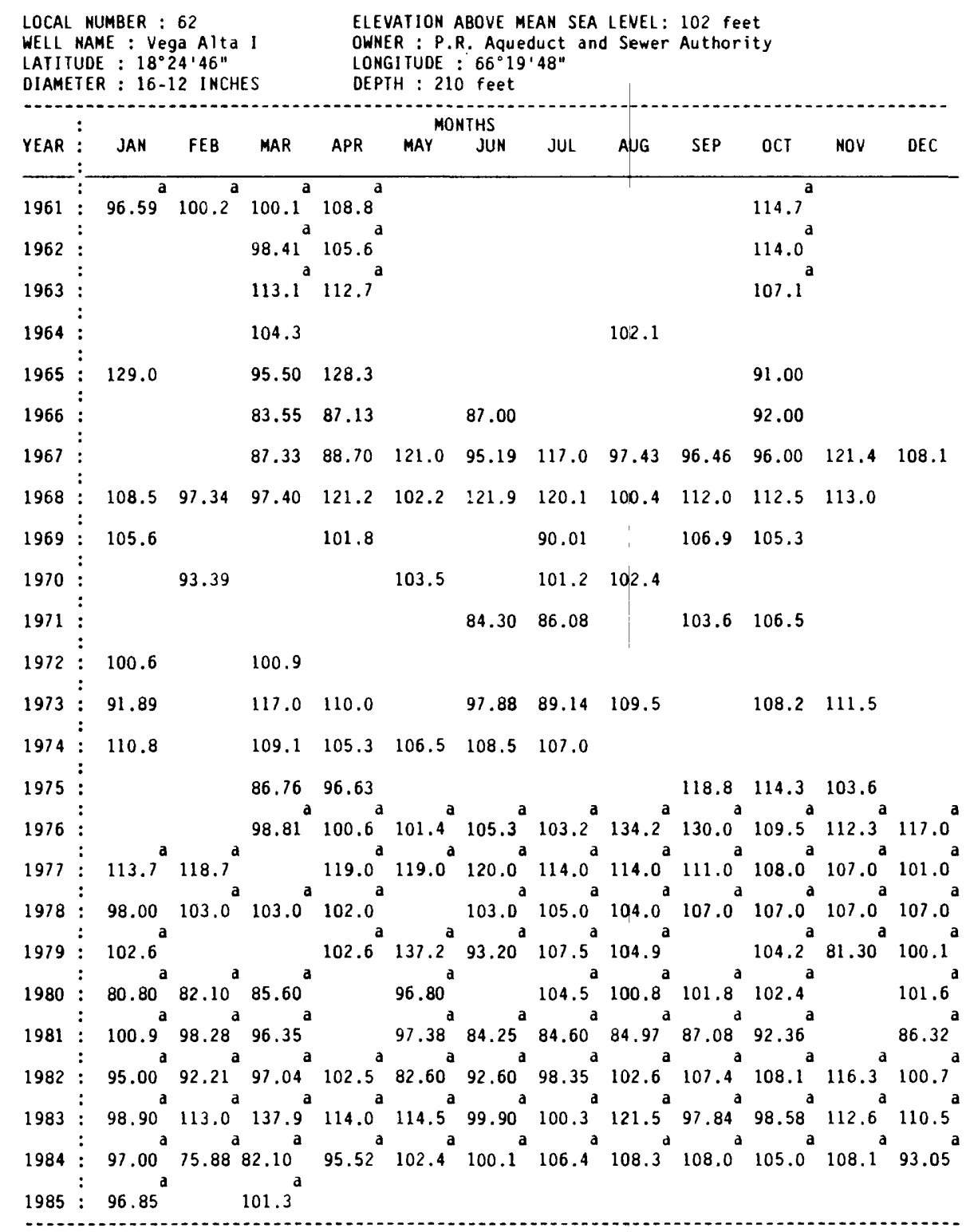

REMARKS: Well discont inued.

a pumping, b recently pumped, e estimated, $h$ tape measurement, $j$ lowest water level recorded, n nearby puaping well 


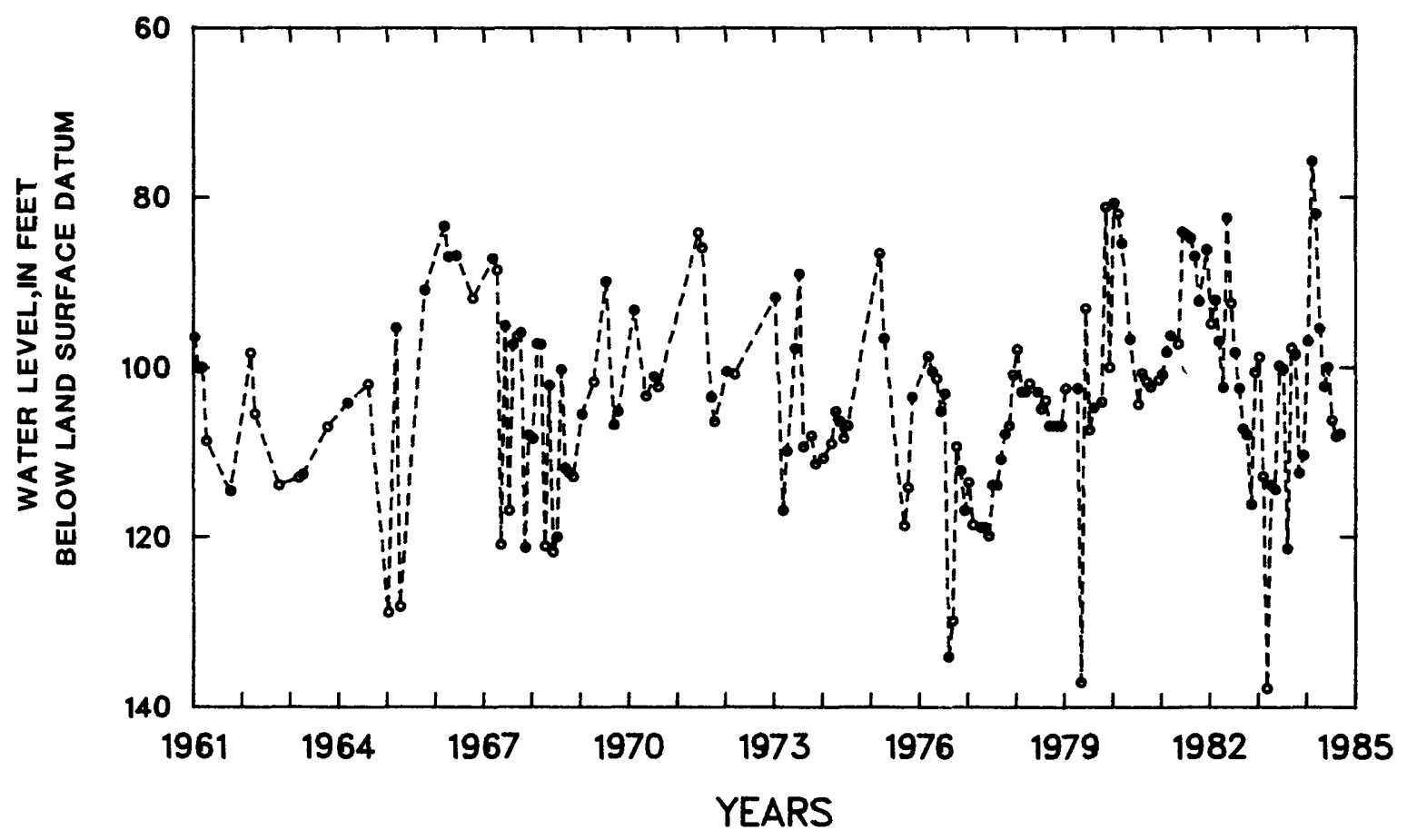

Figure 8.- Ground-water levels at Vega Alta Number 1. local number 62 . 


\begin{tabular}{|c|c|c|c|c|c|c|c|c|c|c|c|c|}
\hline \multicolumn{4}{|c|}{$\begin{array}{l}\text { LOCAL NUMBER : } 63 \\
\text { WELL NAME : Pugnado Afuera } 2 . \\
\text { LATIIUDE : } 18^{\circ} 24^{\circ} 27^{\prime \prime} \\
\text { DIAMETER : } 12 \text { inches }\end{array}$} & $\begin{array}{l}\text { ELE } \\
\text { OWN } \\
\text { LON } \\
\text { DEP }\end{array}$ & $\begin{array}{l}\text { VATION A } \\
\text { ER : P.F } \\
\text { GITUDE } \\
\text { TH: } 200\end{array}$ & $\begin{array}{l}\text { ABOVE M } \\
\text { R. Aque } \\
: 66^{\circ} 25 \\
0 \text { feet }\end{array}$ & $\begin{array}{l}\text { EAN SEA } \\
\text { duct an } \\
\text { ' } 12 \text { " }\end{array}$ & $\begin{array}{l}\text { LEVEL: } \\
\text { Sewer }\end{array}$ & $\begin{array}{l}350 \mathrm{fe} \\
\text { Author }\end{array}$ & $\begin{array}{l}\text { et } \\
\text { ity }\end{array}$ & & \\
\hline & : & & & & MOI & NTHS & & & & & & \\
\hline YEAR & JAN & FEB & MAR & APR & MAY & JUN & JUL & AUG & SEP & $0 \mathrm{CT}$ & NOV & OEC \\
\hline 1959 & $\vdots$ & & 112.1 & & & 94.17 & 97.01 & 103.1 & 107.6 & 111.5 & & 113.7 \\
\hline & : & & & a & & & & & & & & \\
\hline 1960 & $: \quad 113.6$ & & 117.6 & 125.0 & 122.1 & 122.9 & 132.0 & 131.8 & 130.1 & 114.4 & 111.8 & 106.6 \\
\hline & : & a & & & a & & & a & a & a & a & a \\
\hline 1961 & $: 96.98$ & 97.30 & 102.0 & 106.2 & 115.6 & 122.2 & 110.3 & 136.0 & 138.8 & 138.9 & 138.3 & 107.0 \\
\hline & : & & & ${ }^{a}$ & a & a & & $a^{a}$ & a & a & $a$ & a \\
\hline 1962 & : & 106.1 & 107.5 & 109.2 & 113.2 & 121.2 & 129.2 & 128.7 & 132.2 & 133.1 & 132.9 & 138.1 \\
\hline & a & $c^{a}$ & ${ }^{a}$ & ${ }^{a}$ & $a^{a}$ & $a^{a}$ & $a^{a}$ & a & & a & a & $a$ \\
\hline 1963 & $: \quad 138.0$ & 134.6 & 129.2 & 122.7 & 112.2 & 105.4 & 116.4 & 111.8 & & 105.8 & 111.7 & 100.5 \\
\hline 1964 & $\vdots 101.0$ & 97.64 & 105.0 & 105.2 & 105.3 & 116.7 & 116.6 & 112.9 & 131.0 & & 131.7 & 133.6 \\
\hline 1965 & $: 136.0$ & 138.5 & 139.5 & 145.6 & 145.7 & 112.5 & 113.5 & 116.6 & 117.9 & 97.00 & 99.58 & 105.1 \\
\hline 1966 & $: 51.08$ & 54.60 & 55.75 & 77.52 & 81.72 & 82.00 & 81.70 & 81.00 & 80.85 & 80.65 & 80.73 & 107.0 \\
\hline 1967 & $: 109.0$ & 109.2 & $113 . \mathrm{B}$ & 114.8 & 107.5 & 111.0 & 115.6 & 113.7 & 114.0 & 114.1 & 116.6 & 117.0 \\
\hline $196 \mathrm{~B}$ & $: \quad 117.2$ & 116.6 & 116.8 & & 118.1 & 114.4 & 114.2 & 116.8 & 118.0 & 121.2 & 122.8 & \\
\hline 1969 & 92.40 & & 73.94 & 77.06 & 86.47 & & 86.73 & & 106.1 & 101.0 & & \\
\hline 1970 & : & 110.6 & & & & & 117.8 & & & & & \\
\hline
\end{tabular}

REMARKS: Well discont inued.

a pumping, b recently pumped, e estimated, $h$ tape measurement, $j$ lowest water level recorded, n nearby pumping well 


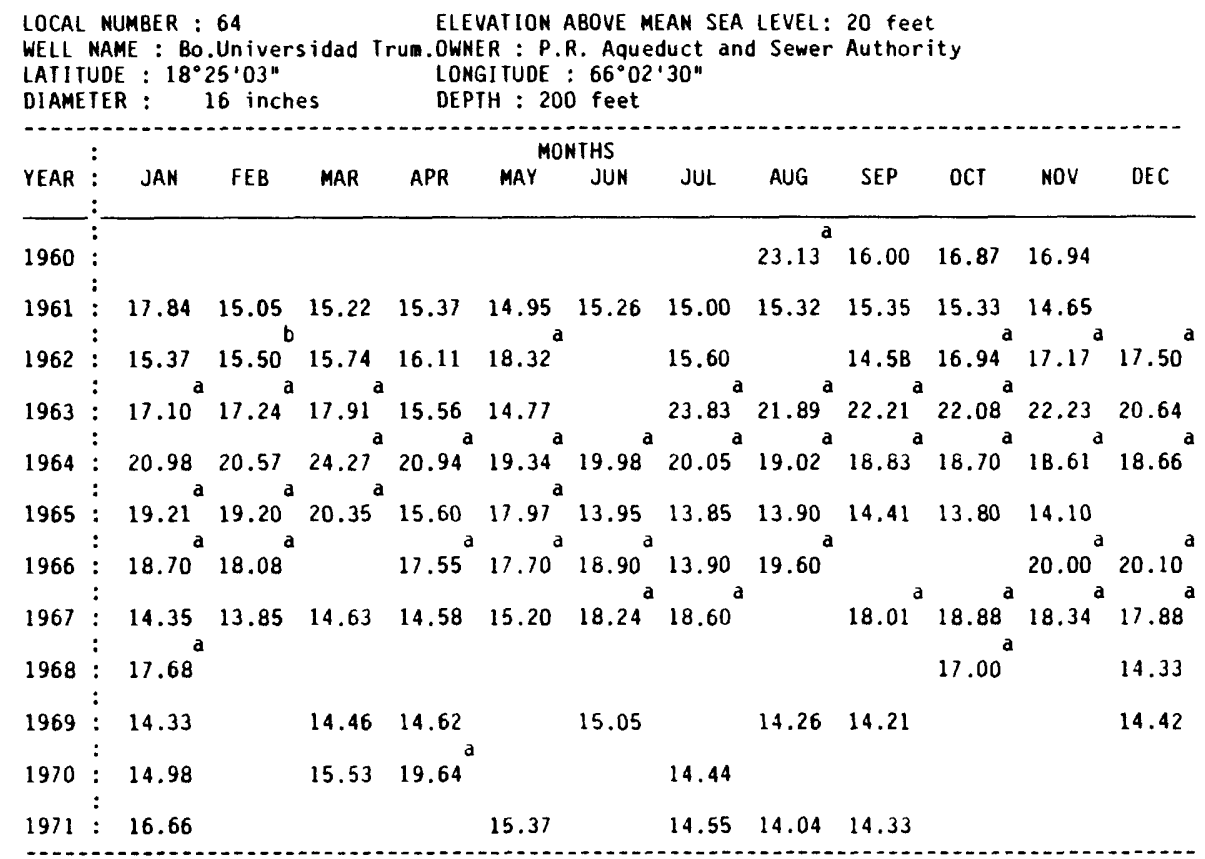

REMARKS: Well discontinued September 1971.

a pumping, b recently pumped, e estimated, $h$ tape measurement, $j$ lowest water level recorded, n nearby pumping well

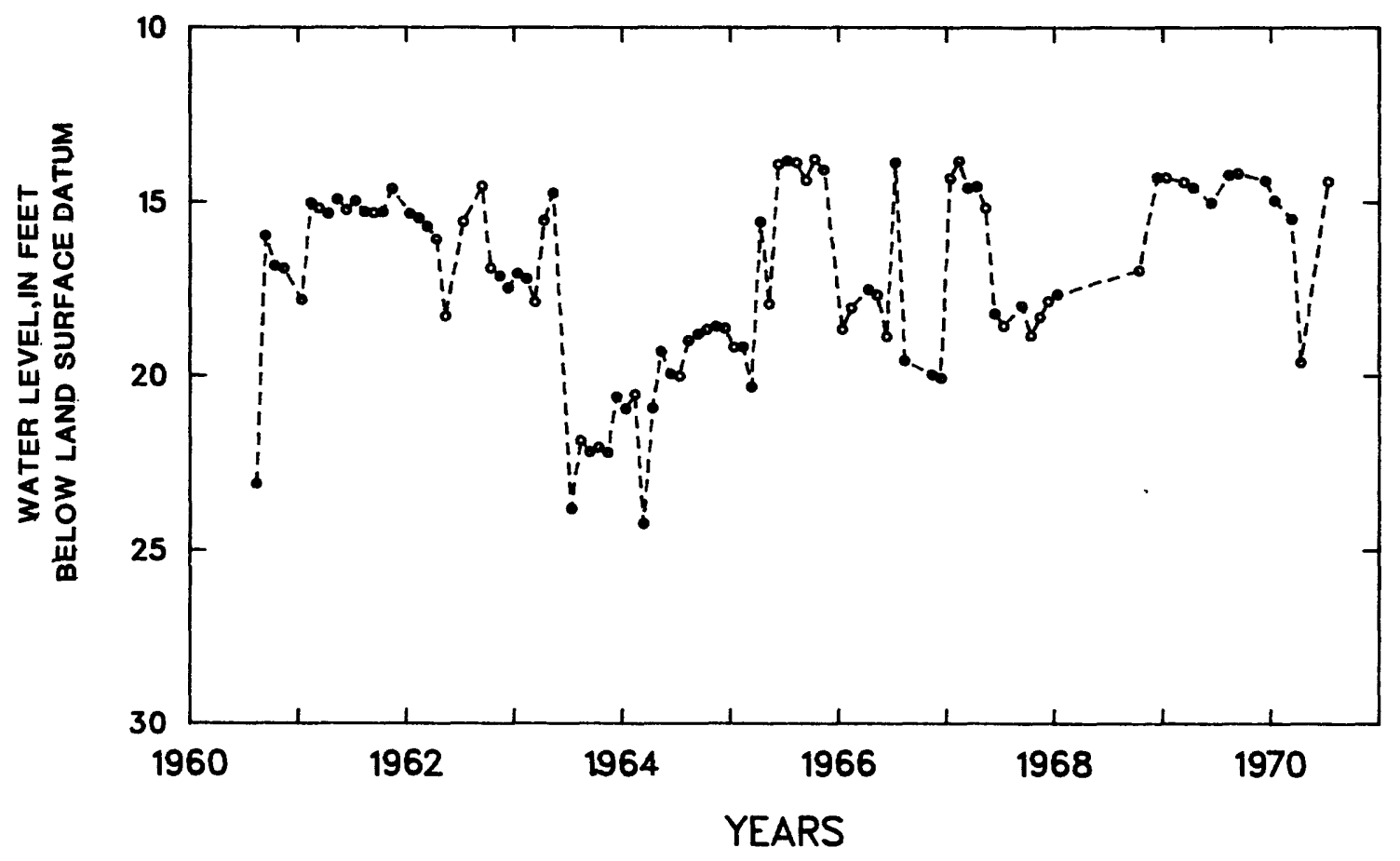

Figure 9.--Ground-water levels of Barrio Universidad TW, local number 64. 


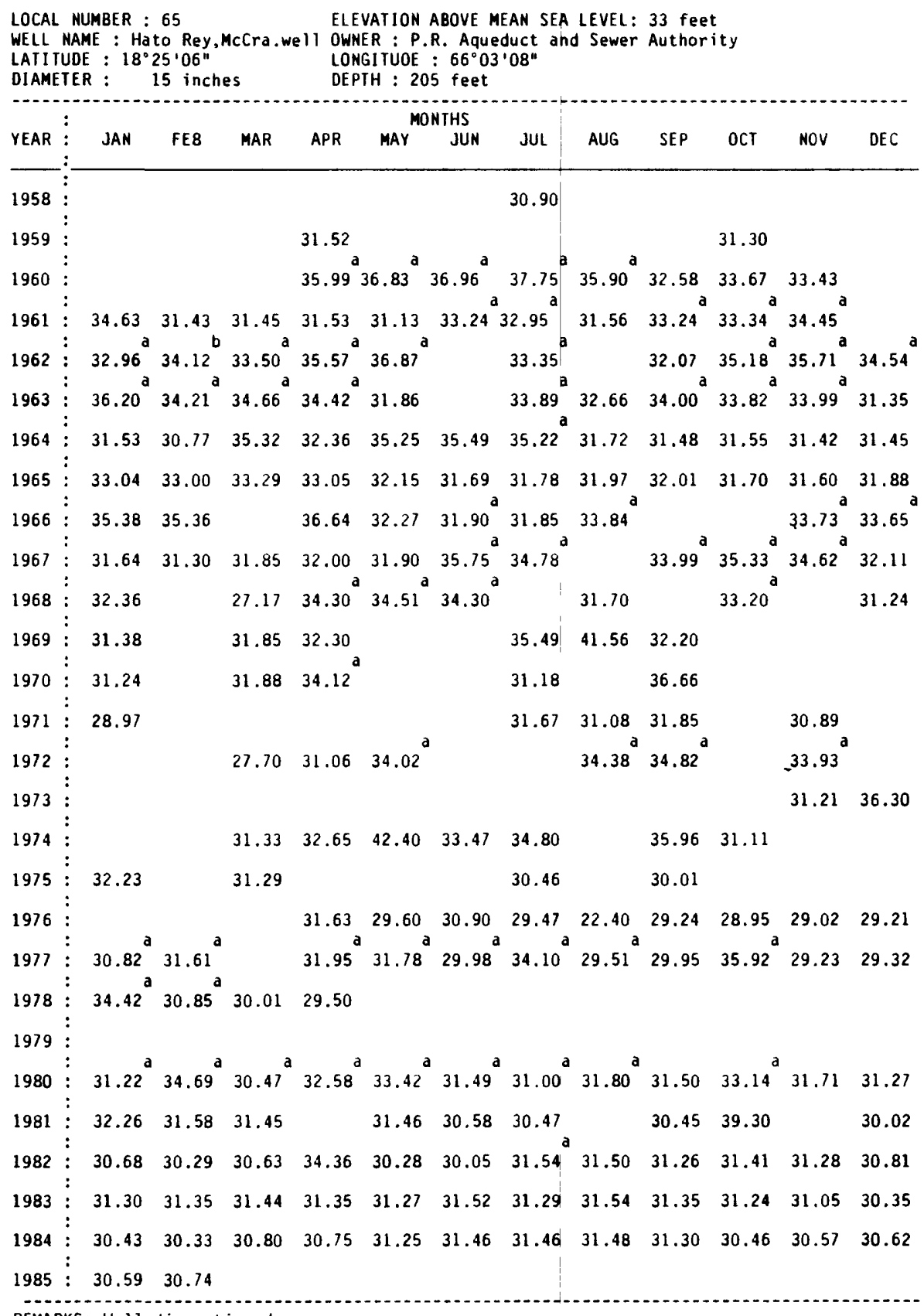

REMARKS: Well discont inued.

a pumping, b recently pumped, e estimated, $h$ tape measurement, $j$ lowest water level recorded, n nearby pumping well 


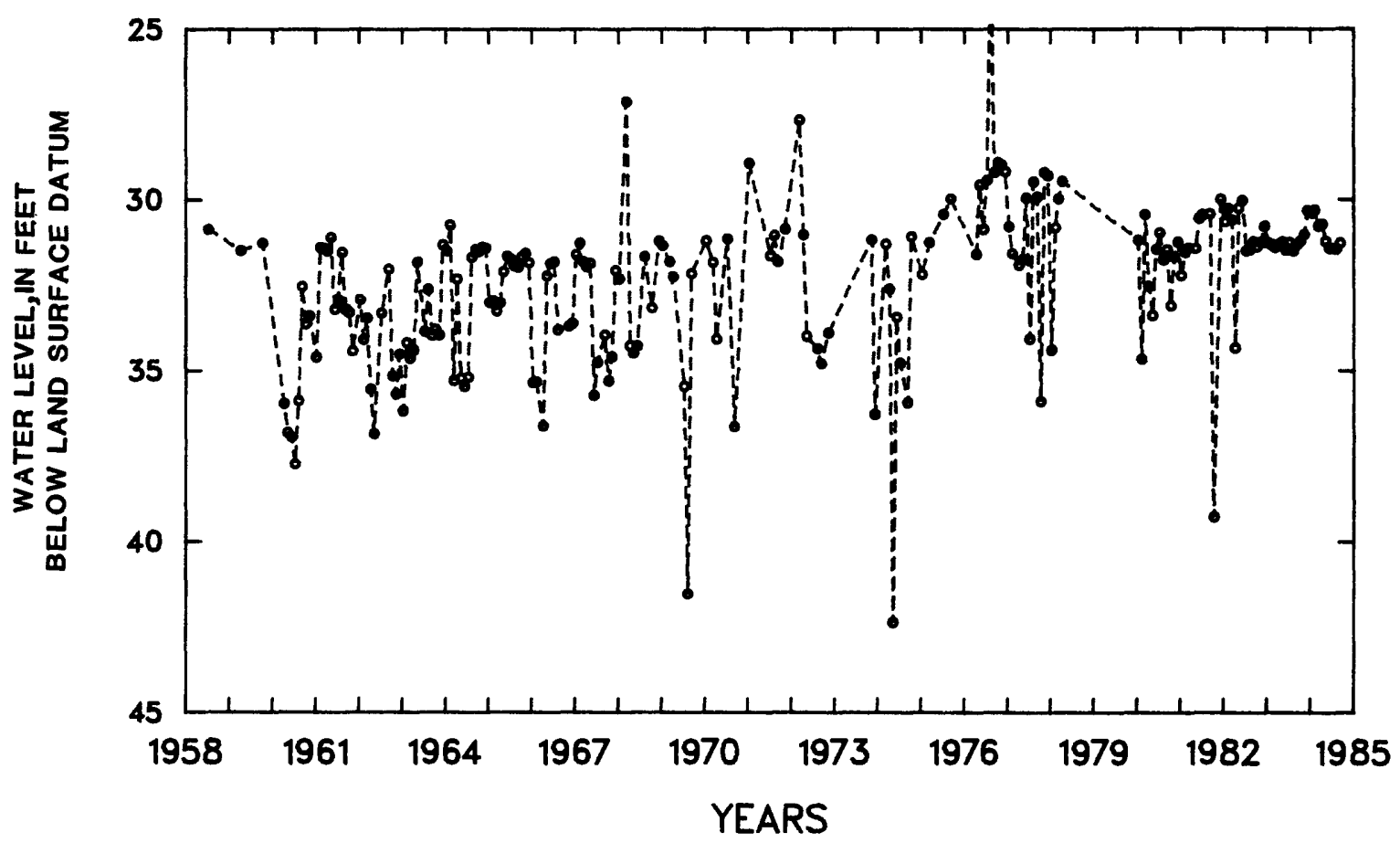

Figure 10.--Ground-water levels at Hato Rey Central, McCracken, local number 65. 


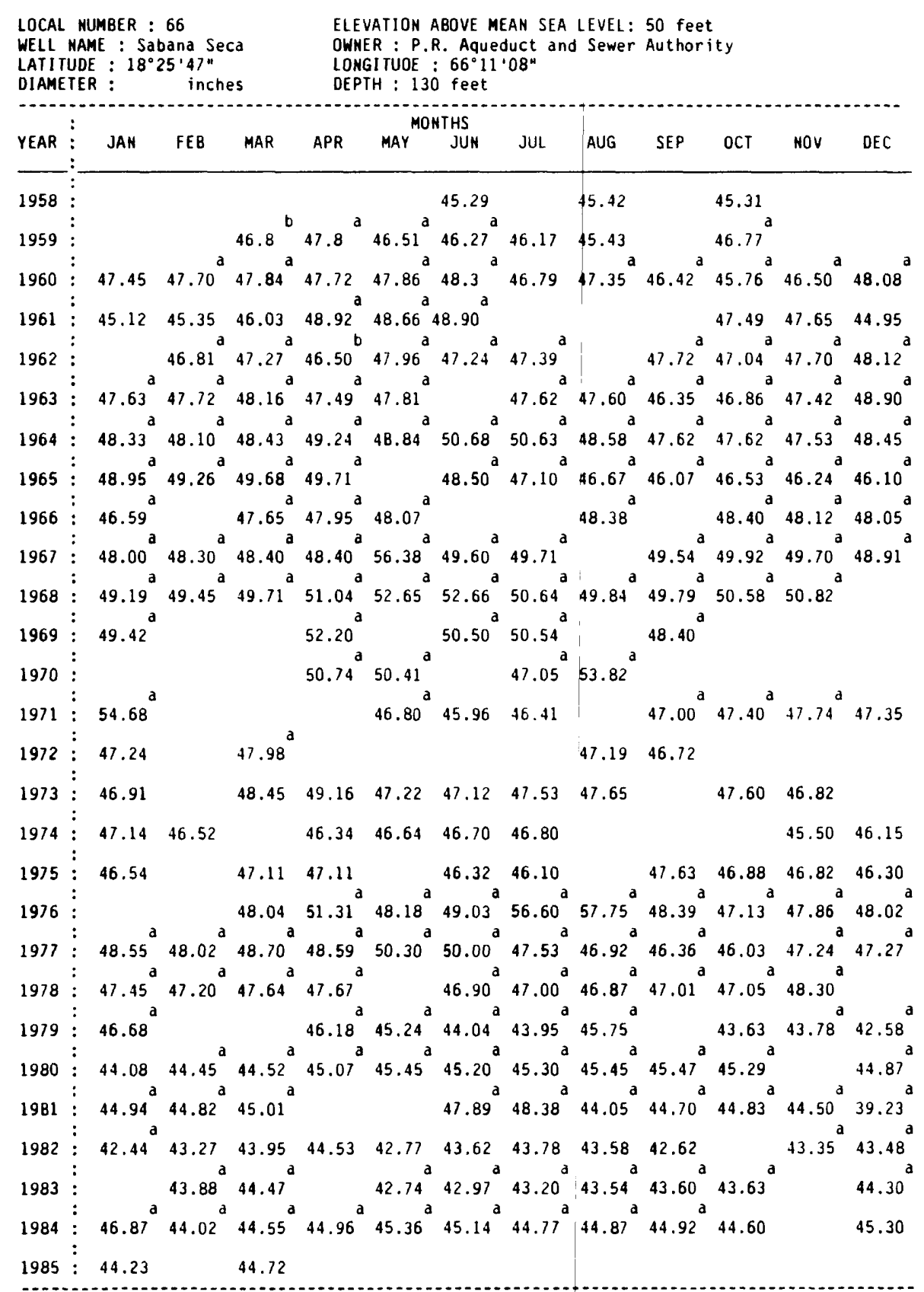

REMARKS: Well discontinued, Apri1, 1985.

a pumping, b recently pumped, e estimated, $h$ tape measurement, $j$ lowest water level recorded, $n$ nearby pumping well 


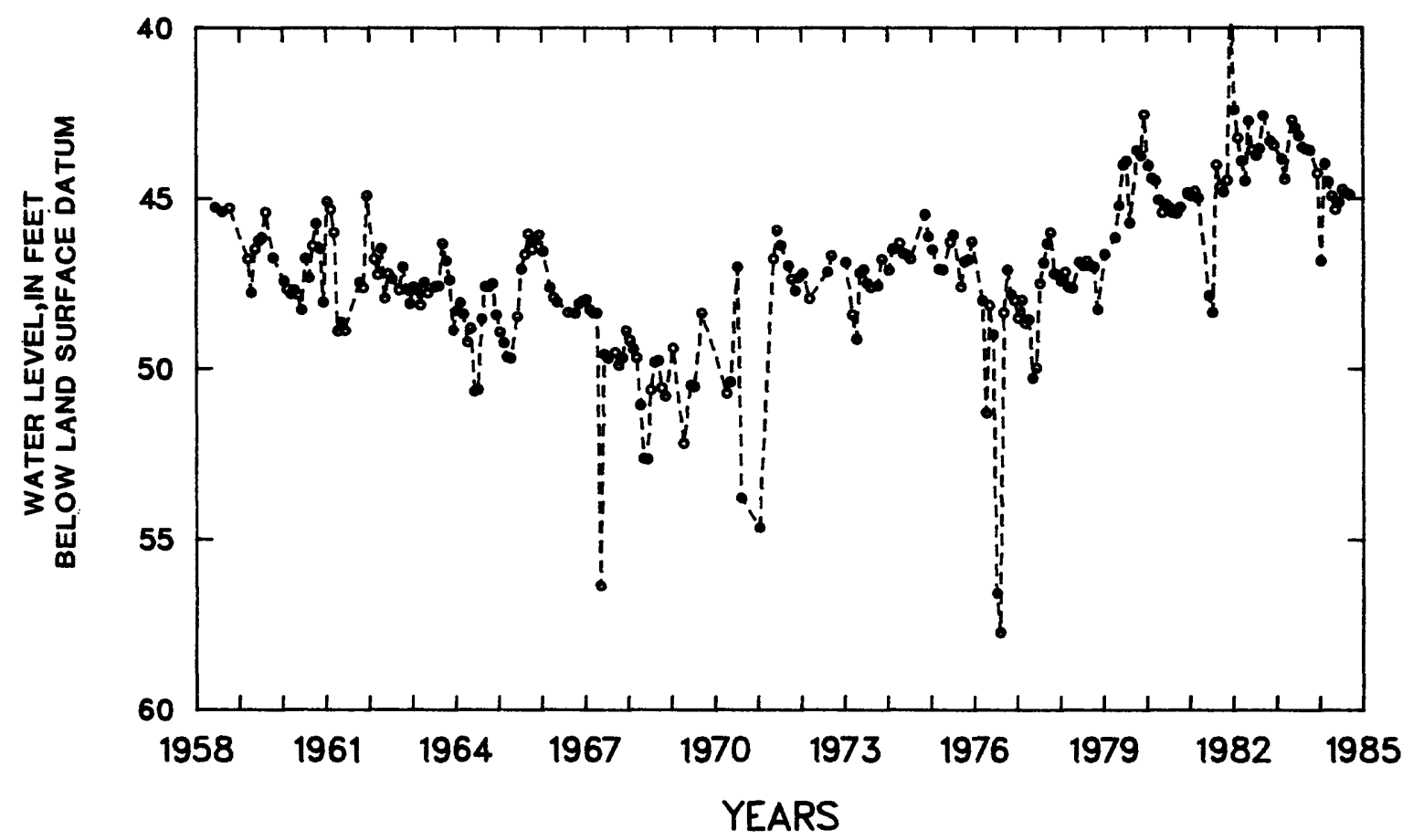

Figure 11.--Ground-water levels at Sabana Seca, local number 66. 


\begin{tabular}{|c|c|c|c|c|c|c|c|c|c|c|c|c|c|}
\hline $\begin{array}{l}\text { LOCAL } \\
\text { WELL } \\
\text { LATIT } \\
\text { DIAME }\end{array}$ & $\begin{array}{l}\text { NA } \\
\text { MA } \\
\text { TE }\end{array}$ & $\begin{array}{l}\text { JMBER : } \\
\text { AE: Can } \\
\text { : } 18^{\circ}\end{array}$ & $\begin{array}{l}67 \\
\text { mpanill } \\
25^{\prime} 1 \text { B }^{\prime \prime} \\
12 \text { inch }\end{array}$ & & $\begin{array}{l}\text { ELE } \\
\text { OWN } \\
\text { LON } \\
\text { DEP }\end{array}$ & $\begin{array}{l}\text { VATION } \\
\text { ER : P. } \\
\text { GITUDE } \\
\text { TH : } 30\end{array}$ & $\begin{array}{l}\text { ABOVE } \\
\text { R. Aque } \\
: 66^{\circ} 14 \\
0 \text { feet }\end{array}$ & $\begin{array}{l}\text { IN SEA } \\
\text { ict ar } \\
12 "\end{array}$ & $\begin{array}{l}\text { LEVEL: } \\
\text { Sewer }\end{array}$ & $\begin{array}{l}39 \mathrm{fe} \\
\text { Autho }\end{array}$ & & & \\
\hline$\cdots$ & : & & & & & MO & NTHS & & & & & & \\
\hline YEAR & $:$ & JAN & FEB & MAR & APR & MAY & JUN & JUL & AUG & SEP & OCT & NOV & DEC \\
\hline 1959 & $:$ & & & $36.30^{\mathrm{a}}$ & $37.30^{a}$ & $34.90^{a}$ & $34.20^{a}$ & $35.10^{\mathrm{a}}$ & $35.30^{\mathrm{a}}$ & & $39.30^{\mathrm{a}}$ & & $38.30^{\circ}$ \\
\hline & $:$ & a & & & a & & & & a & a & a & a & a \\
\hline 1960 & : & 38.20 & $38.60^{\circ}$ & 38.70 & 39.60 & 38.60 & 40.80 & 39.80 & 37.90 & 38.20 & 34.90 & 35.80 & 35.50 \\
\hline & : & a & $\operatorname{ar}^{a}$ & & & & $a^{a}$ & & a & & a & a & $a$ \\
\hline 1961 & : & 35.80 & 35.90 & 29.74 & 36.50 & 36.60 & 36.50 & & 36.00 & 35.40 & 36.60 & 37.33 & 33.28 \\
\hline & : & & & & & & a & a & & a & a & a & a \\
\hline 1962 & : & & 35.45 & 36.38 & 29.63 & 37.10 & 36.80 & 36.27 & & 36.03 & 36.90 & 36.90 & 38.18 \\
\hline & : & a & & & a & & & a & a & a & a & a & a \\
\hline 1963 & $:$ & 36.90 & 36.62 & 36.19 & 35.40 & 33.75 & & 37.96 & 39.08 & 37.91 & 38.39 & 36.10 & 36.46 \\
\hline & : & & & & a & a & a & a & a & a & a & a & a \\
\hline 1964 & : & 36.59 & 36.83 & 37.68 & 37.70 & 37.57 & 39.88 & 39.95 & 41.99 & 38.35 & 36.90 & 36.75 & 43.33 \\
\hline & $:$ & & & & & & & & & & & & \\
\hline 965 & $:$ & 35.65 & 44.87 & 45.78 & & & & & & & & & \\
\hline & : & & & & $a^{a}$ & & a & a & a & & & & \\
\hline 1966 & $:$ & & & 42.00 & 42.98 & 34.43 & 41.96 & 34.75 & 34.70 & & 34.45 & 33.89 & 33.70 \\
\hline 1967 & $:$ & 33.55 & 33.52 & 33.21 & 33.00 & 35.23 & $49.90^{\circ}$ & 38.04 & & 36.64 & 38.39 & 37.52 & 36.66 \\
\hline 1968 & : & 36.77 & 38.12 & 36.13 & 36.58 & 37.15 & 37.24 & 35.14 & 32.70 & 34.13 & 35.32 & 37.33 & \\
\hline 1969 & : & 35.13 & & 33.95 & 35.13 & & 34.73 & 35.60 & & 35.90 & & & \\
\hline 1970 & $\vdots$ & & & & & 37.79 & & 35.91 & $\$ 4.44$ & & & & \\
\hline 1971 & : & & & & & & 35.37 & 35.68 & 1 & 36.43 & 36.98 & & \\
\hline 1972 & : & 37.79 & & 38.09 & & & & & 37.90 & 37.47 & & & \\
\hline 1973 & $\vdots$ & 36.87 & & 37.40 & 36.30 & & 34.22 & 35.93 & 36.77 & & & 37.10 & \\
\hline 1974 & $\vdots$ & 43.64 & 38.05 & & 39.09 & 39.13 & & 39.74 & & & & & 34.51 \\
\hline 1975 & $\vdots$ & 35.46 & & 36.38 & 38.47 & & 37.51 & 38.84 & & 38.15 & 36.97 & 35.69 & 33.52 \\
\hline 1976 & $\vdots$ & & & 36.30 & 36.85 & 36.00 & 37.95 & 36.90 & 38.15 & 38.55 & 38.35 & 37.93 & 38.26 \\
\hline & : & & & & & & & & & & & & \\
\hline 1977 & : & 38.32 & 38.40 & 38.83 & 38.69 & 39.45 & 38.97 & 38.77 & 38.51 & 39.10 & 38.28 & 38.08 & 37.62 \\
\hline 1978 & : & 38.28 & 37.50 & 37.71 & 38.21 & & & 38.49 & 38.68 & 38.61 & 39.13 & & \\
\hline 1979 & $\vdots$ & 38.97 & & & 33.97 & 33.92 & 31.20 & 30.68 & 33.06 & 29.79 & 28.50 & 32.85 & 31.39 \\
\hline 1980 & $\vdots$ & 33.99 & 33.34 & 34.26 & 34.28 & 35.92 & 35.30 & 35.39 & 35.43 & 35.45 & 35.89 & & 34.85 \\
\hline 1981 & $\vdots$ & 35.11 & 35.34 & 33.90 & & 34.02 & 33.24 & 34.15 & 33.85 & 33.77 & 34,25 & 33.72 & 33.91 \\
\hline & $:$ & & & & & & & & & & & & \\
\hline 1982 & $:$ & 29.42 & 31.68 & 32.64 & 34.27 & 33.04 & 33.60 & 34.02 & 33.80 & 33.59 & 34.93 & & 33.38 \\
\hline 983 & : & & 34.10 & & & & & 33 & & 25 & 34.31 & & 34.12 \\
\hline
\end{tabular}

a pumping, b recently pumped, e estimated, $h$ tape measurement, $j$ lowest water level recorded. n nearby pumping well 
THIS PAGE WAS LEFT BLANK INTENTIONALLY 


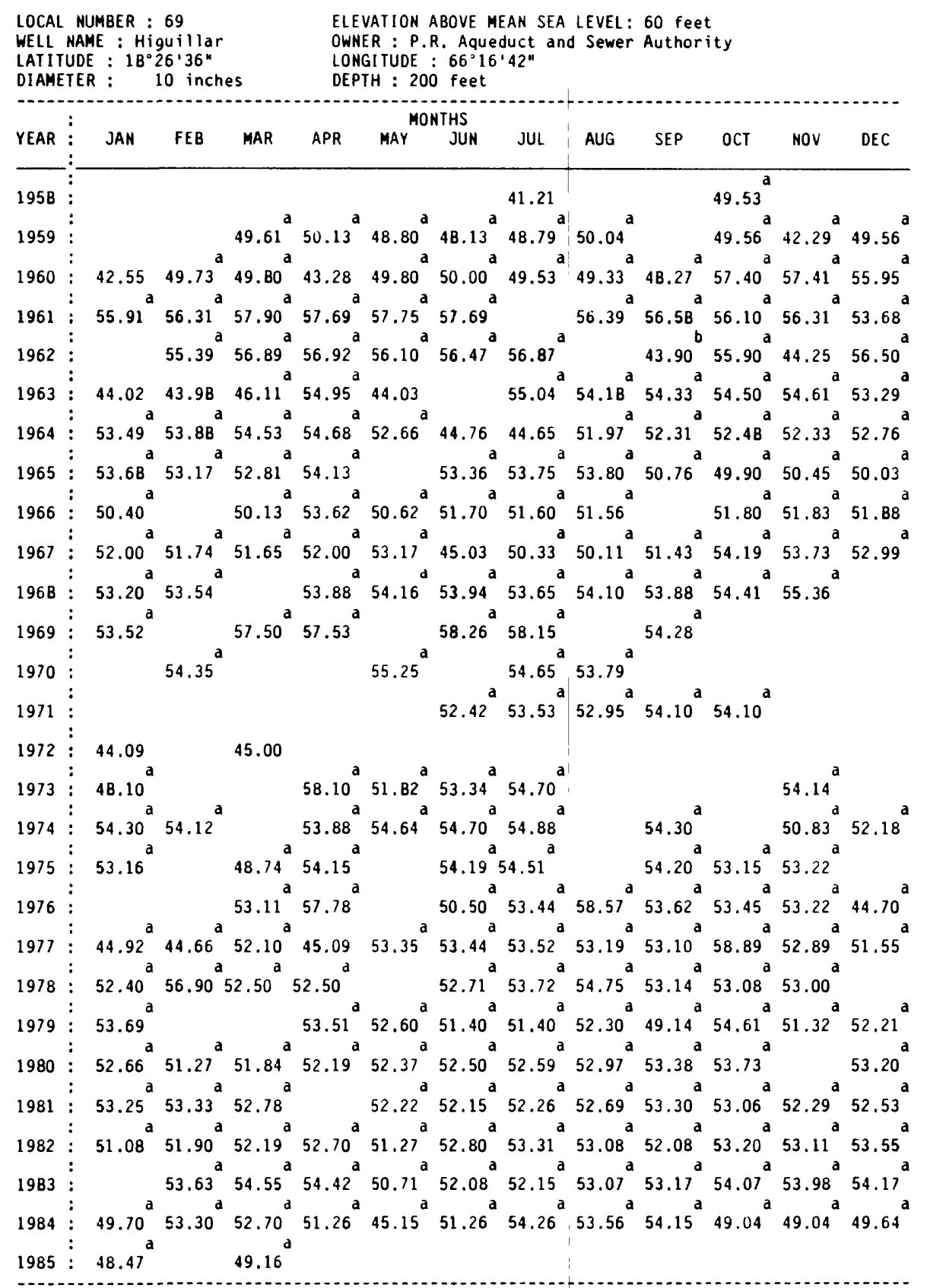

a pumping, b recently pumped, e estimated, $h$ tape measurement, $j$ lowest water level recorded, n nearby pumping well 


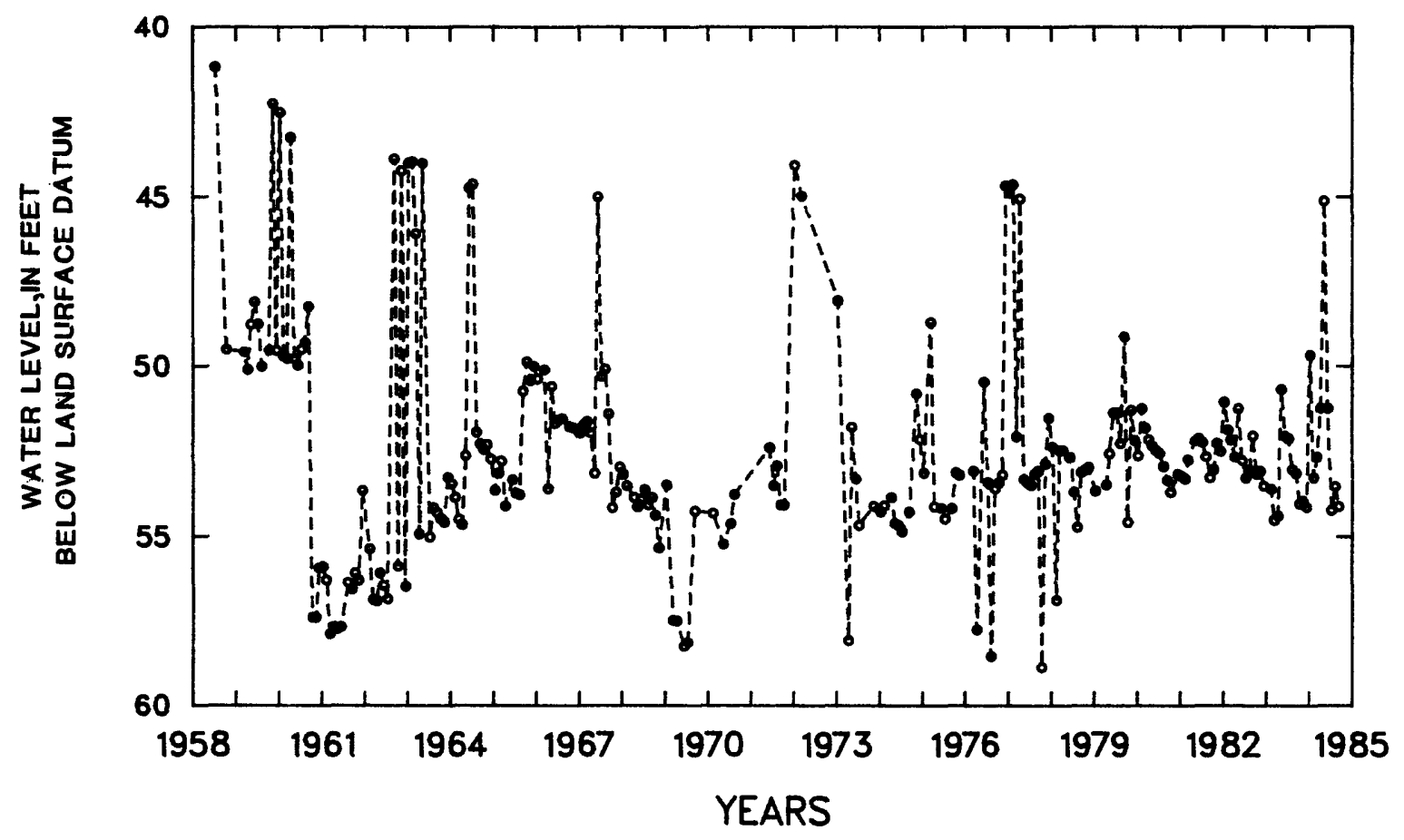

Figure 12.--Ground-water levels at Higuillar, local number 69. 


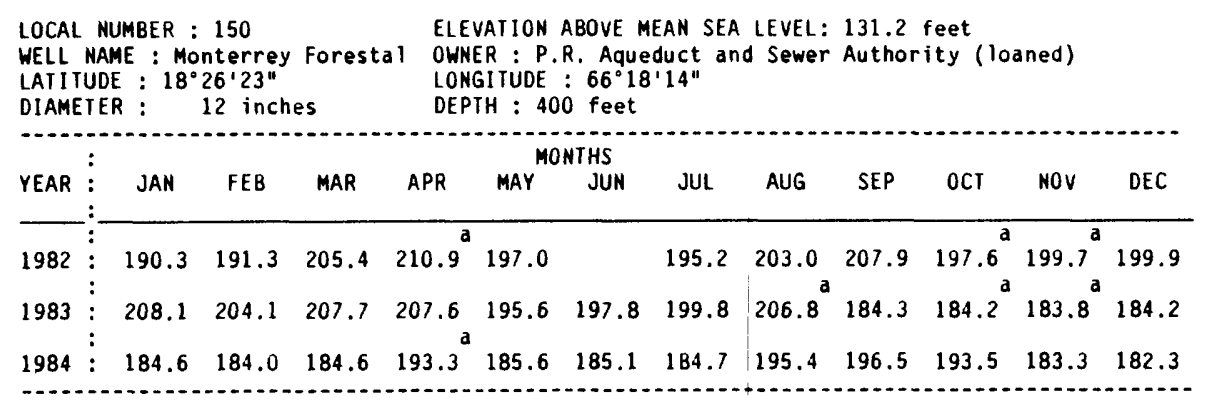

\begin{tabular}{|c|c|c|c|c|c|c|c|c|c|c|c|c|c|}
\hline \multirow{3}{*}{$\begin{array}{l}\text { LOCAL } \\
\text { WELL } \\
\text { LATIT } \\
\text { DIAME } \\
\text { YEAR }\end{array}$} & \multicolumn{4}{|c|}{$\begin{array}{l}\text { MUMBER : } 70 \\
\text { NAME : } 80^{\circ} \text {. Sabana Hoyos } \\
\text { JUDE : } 18^{\circ} 26^{\prime} 47^{\prime \prime} \\
\text { ETER : } 8.0 \text { inches }\end{array}$} & $\begin{array}{l}\text { ELE } \\
\text { OWN } \\
\text { LON } \\
\text { DEP }\end{array}$ & $\begin{array}{l}\text { VATION } \\
\text { ER : P. } \\
\text { GITUDE } \\
\text { TH : } 90\end{array}$ & $\begin{array}{l}\text { A80VE } \\
\text { R. Aque } \\
: 66^{\circ} 20 \\
\text { feet }\end{array}$ & $\begin{array}{l}\text { IEAN SEA } \\
\text { duct an } \\
\text { ' } 27 \%\end{array}$ & $\begin{array}{l}\text { LEVEL: } \\
\text { d Sewer }\end{array}$ & $\begin{array}{l}49 \text { fee } \\
\text { Author }\end{array}$ & ity & & \\
\hline & : & & & & & MO & ITHS & & & & & & -- \\
\hline & : & JAN & FEB & MAR & $A P R$ & MAY & JUN & JUL & AUG & SEP & $\mathrm{OCT}$ & NOV & DEC \\
\hline 1960 & : & & 27.09 & 27.33 & 27.57 & 27.46 & 27.65 & 27.62 & 27.10 & 27.00 & 26.69 & 27.14 & 26.74 \\
\hline 1961 & $\vdots$ & 26.09 & 26.79 & 27.22 & 27.47 & 27.54 & 27.78 & 27.73 & 27.32 & 27.35 & 27.55 & 27.57 & 25.85 \\
\hline 1962 & : & 25.52 & 26.21 & 26.75 & 27.62 & 27.16 & 27.19 & 27.24 & 27.30 & 27.35 & 27.40 & 27.55 & 27.71 \\
\hline 1963 & : & 27.27 & 27.45 & 27.73 & 27.06 & 27.20 & 27.13 & 27.24 & 27.06 & 26.89 & 26.81 & 26.90 & 26.50 \\
\hline 1964 & $\vdots$ & & 27.23 & 27.49 & 27.51 & 27.85 & 27.96 & 27.79 & 27.82 & 27.63 & 27.79 & 28.02 & 28.19 \\
\hline 1965 & : & 28.37 & & & & & & & & & & 25.92 & 25.96 \\
\hline 1966 & $\vdots$ & $25.30^{e}$ & 25.80 & 25.91 & 25.57 & 25.88 & 25.74 & 26.23 & 26.45 & 26.55 & 26.63 & 26.63 & 26.64 \\
\hline 1967 & : & 26.92 & 27.51 & 27.95 & $2 B .59$ & & 29.05 & 29.05 & 28.90 & 28.94 & 28.98 & 28.68 & 28.89 \\
\hline 1968 & $\vdots$ & 28.92 & 29.12 & 28.97 & 29.32 & 29.40 & 29.22 & 2B. 89 & 28.90 & 28.69 & 29.06 & 29.14 & 27.64 \\
\hline 1969 & : & 27.91 & 27.52 & 27.87 & 28.18 & 28.25 & 28.00 & 28.10 & 28.34 & 28.44 & 28.90 & 28.86 & \\
\hline 1970 & $\vdots$ & & 28.10 & 28.19 & & 28.34 & 2 B. 53 & 28.45 & 28.40 & 27.94 & 27.93 & & \\
\hline 1971 & $\vdots$ & 24.98 & 25.47 & 26.15 & & 26.89 & 27.37 & $27.74^{e}$ & 27.84 & 28,14 & 28.24 & 28.46 & 28.67 \\
\hline 1972 & $\vdots$ & 28.60 & 28.66 & 28.57 & 28.67 & 28.87 & 29.07 & 29.08 & 29.07 & 29.03 & 29.00 & 28.64 & 28.70 \\
\hline 1973 & $\vdots$ & & & 29.05 & 29.21 & 27.71 & 28.02 & & 28.76 & 28.76 & 28.84 & 29.97 & 29.97 \\
\hline 1974 & : & 29.09 & 28.88 & 29.22 & 29.36 & 29.45 & 29.55 & 29.73 & 29.72 & 29.88 & 29.60 & 26.39 & 27.41 \\
\hline 1975 & $\vdots$ & 28.40 & 28.86 & 29.14 & $29.5 B$ & 29.83 & 30.06 & 31.10 & 31.08 & 30.02 & 28.87 & 28.68 & 26.78 \\
\hline 1976 & : & 28.00 & 28.45 & & 28.81 & 29.04 & 29.21 & 29.25 & 29.48 & 29.53 & 29.48 & 29.59 & 29.67 \\
\hline 1977 & : & 29.82 & 29.80 & 29.97 & 30.09 & 30.26 & 30.37 & 30.42 & 30.28 & 30.01 & 29.94 & 29.38 & 28.37 \\
\hline 1978 & $\vdots$ & 29.24 & 29.33 & 29.42 & 29.34 & 29.06 & 29.51 & 29.77 & 29.94 & 29.96 & 30.07 & 29.98 & 29.88 \\
\hline 1979 & $\vdots$ & 30.30 & 30.66 & 30.28 & 30.06 & 29.83 & 27.92 & 27.86 & 28.06 & 26.10 & 26.75 & 26.94 & 26.62 \\
\hline & : & $e^{e}$ & & & & & & & & & & & \\
\hline 1980 & : & 27.14 & 27.67 & 28.03 & 28.39 & 28.79 & 28.83 & 29.01 & 29.16 & 29.23 & 29.18 & 29.22 & 29.27 \\
\hline 1981 & $\vdots$ & 29.22 & 29.35 & 29.48 & 28.72 & 28.72 & 27.96 & 28.23 & 28.17 & 28.51 & 28.79 & 28.60 & 28.34 \\
\hline 1982 & $\vdots$ & 26.34 & 26.89 & 27.58 & 28.05 & 28.10 & 27.73 & 28.03 & 28.33 & 28.32 & 28.70 & 28.81 & 28.99 \\
\hline 1983 & : & 28.85 & 29.27 & 29.56 & 29.67 & 29.05 & 29.14 & 29.35 & 29.46 & 29.27 & 29.07 & 28.96 & 29.18 \\
\hline 1984 & $\vdots$ & 29.40 & 29.46 & 29.64 & 30.02 & 30.15 & 30.03 & 29.74 & 29.66 & 29.74 & 29.13 & 29.00 & 28.40 \\
\hline 1985 & $\vdots$ & $2 B .39$ & 28.86 & 28.86 & 28.93 & 28.86 & 28.34 & 28.80 & 29.03 & 28.99 & & & \\
\hline
\end{tabular}




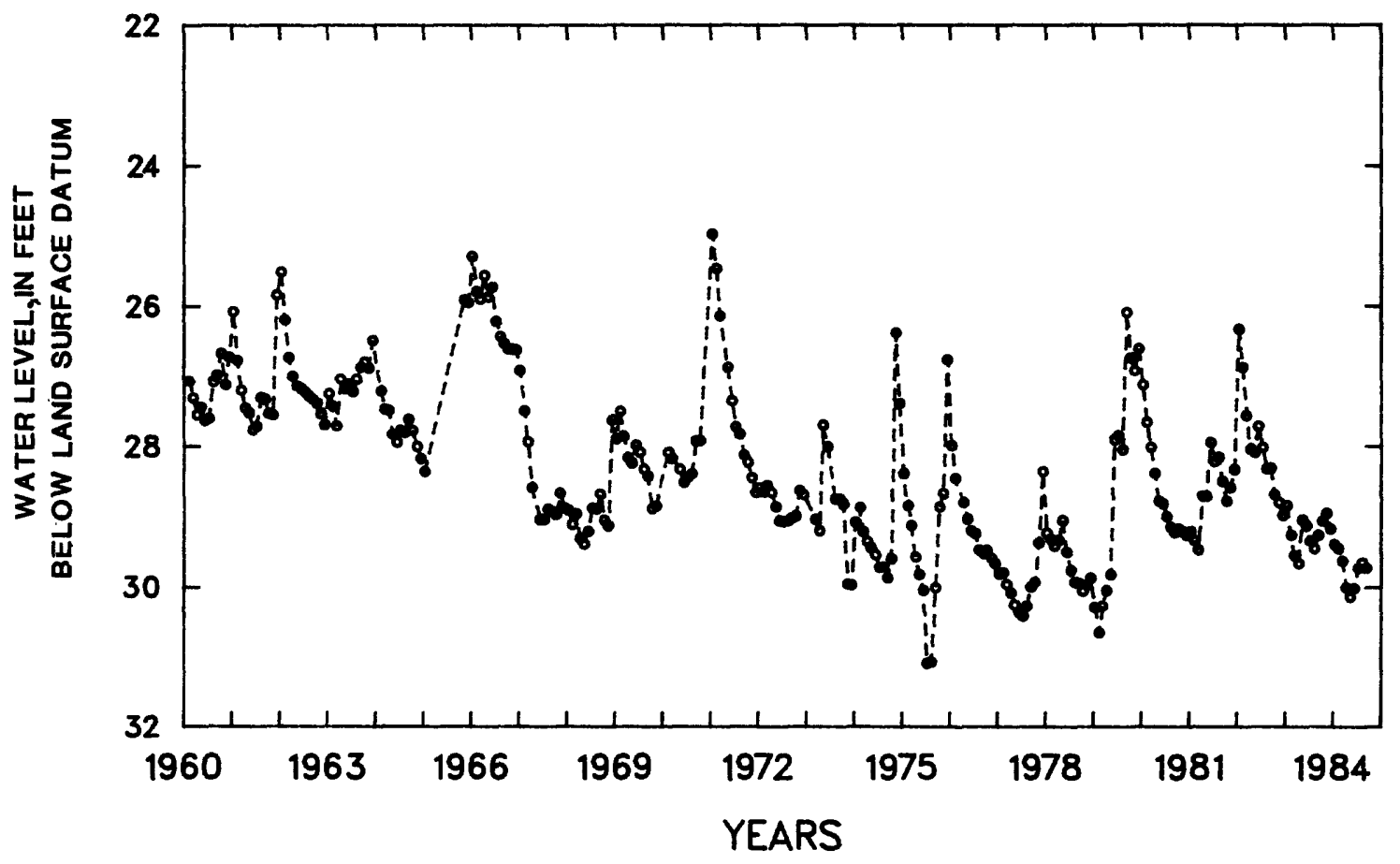

Figure 13.--Ground-water levels at Barrio Sabana Hoyos, 


\begin{tabular}{|c|c|c|c|c|c|c|c|c|c|c|c|c|}
\hline $\begin{array}{l}\text { LOCAL } \\
\text { WELL } \\
\text { LATI } \\
\text { DIAME }\end{array}$ & $\begin{array}{l}\text { MUMBER : } \\
\text { NAME : La } \\
\text { TUDE : } 18^{\circ} \\
\text { TER : } 20\end{array}$ & $\begin{array}{l}155 \\
\text { Trocha } \\
26^{\prime} 12^{\prime \prime} \\
16 \text { inch }\end{array}$ & & $\begin{array}{l}\text { ELE } \\
\text { OWI } \\
\text { LOI } \\
\text { DE }\end{array}$ & $\begin{array}{l}\text { VATION } \\
\text { ER : P. } \\
\text { GITUOE } \\
T H: 20\end{array}$ & $\begin{array}{l}\text { ABOVE } \\
\text { R. Aque } \\
: 66^{\circ} 22 \\
0 \text { feet }\end{array}$ & $\begin{array}{l}\text { EAN SEA } \\
\text { duct an } \\
\text { '54" }\end{array}$ & $\begin{array}{l}\text { L'EVEL } \\
\text { o Sewer }\end{array}$ & $\begin{array}{l}49.20 \\
\text { Author }\end{array}$ & $\begin{array}{l}\text { eet } \\
\text { ty }\end{array}$ & & \\
\hline YEAR & : & FEB & MAR & APR & MAY ${ }^{\text {MO }}$ & $\begin{array}{l}\text { NTHS } \\
\text { JUN }\end{array}$ & JUL & AUG & SEP & OCT & NOV & $D E C$ \\
\hline 1982 & $: \quad 32.23$ & 34.02 & 34.38 & 33.56 & 33.26 & 33.48 & & & & & & \\
\hline 1983 & $\vdots$ & & & 34.27 & 33.95 & 34.08 & 34.00 & 34.32 & 34.60 & 34.10 & 34.02 & 34.38 \\
\hline 1984 & $: \quad 34.40$ & 33.26 & 33.48 & 34.60 & 33.95 & 34.08 & 34.51 & 34.32 & 34.60 & 34.22 & 34.00 & 33.92 \\
\hline
\end{tabular}

\begin{tabular}{|c|c|c|c|c|c|c|c|c|c|c|c|c|}
\hline \multicolumn{4}{|c|}{$\begin{array}{l}\text { IOCAL NUM8ER: } 167 \\
\text { WELL NAME : U.S.G.S. No. } 3 \\
\text { LATITUDE : } 18^{\circ} 26^{\prime} 56^{\prime \prime} \\
\text { DIAMETER : } 6 \text { inches }\end{array}$} & $\begin{array}{l}\text { ELE } \\
\text { OWN } \\
\text { LON } \\
\text { DEP }\end{array}$ & $\begin{array}{l}\text { IATION } \\
\text { R : P. } \\
\text { IITUDE } \\
\text { TH : } 23\end{array}$ & $\begin{array}{l}\text { ABOVE } \\
\text { R. Land } \\
66^{\circ} 22 \\
\text { feet }\end{array}$ & $\begin{array}{l}\text { AN SEA } \\
\text { Author } \\
15^{\prime \prime}\end{array}$ & $\begin{array}{l}\text { LEVEL: } \\
\text { ity }\end{array}$ & $65.6 \mathrm{f}$ & eet & & \\
\hline YEAR & : & FE8 & MAR & APR & MAY & $\begin{array}{c}\text { YTHS } \\
\text { JUN }\end{array}$ & JUL & AUG & SEP & OCT & NOV & DEC \\
\hline 1982 & $: 13.07$ & 13.22 & 14.40 & 14.38 & 14.05 & 13.90 & 14.80 & 14.50 & 14.66 & $14.95^{n}$ & $15.24^{n}$ & $15.47^{n}$ \\
\hline 1983 & $: 15.12^{n}$ & $15.30^{n}$ & $15.90^{n}$ & $16.00^{n}$ & $15.55^{\prime \prime}$ & $15.70^{\circ}$ & $15.50^{\prime \prime}$ & $16.90^{\prime \prime}$ & $14.93^{n}$ & 15.13 & 14.95 & 15.14 \\
\hline 1984 & $: 14.42$ & 15.19 & & & 15.92 & 15.52 & 15.60 & 15.54 & 15.96 & 15.12 & 14.63 & 14.75 \\
\hline
\end{tabular}

REMARKS: Well discont inued.

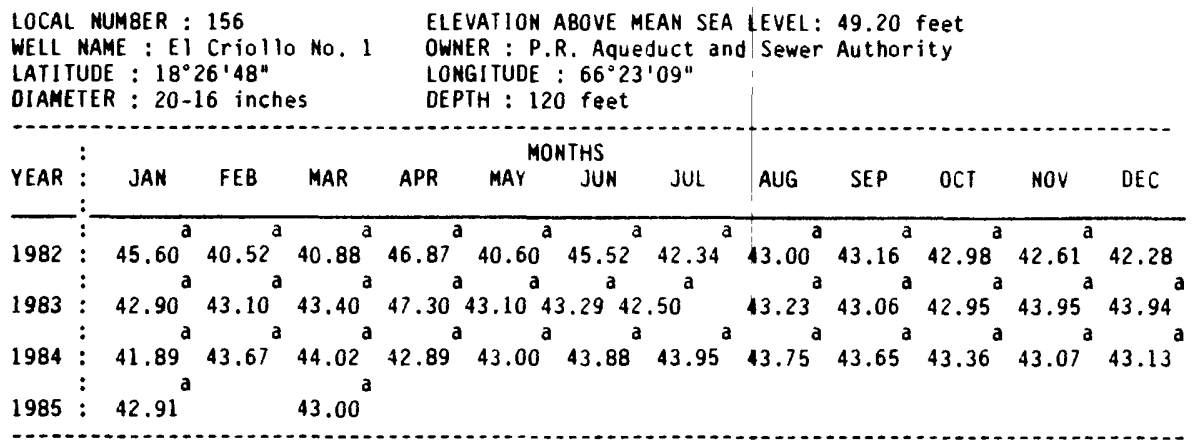

REMARKS: Well discontinued.

LOCAL MUMBER : 151 ELEVATION ABOVE MEAN SEA LEVEL: 19.7 feet

WELL NAME : Rice Progra No. 3 OWNER : AFDA-P.R. Department of Agriculture

LATITUDE : $18^{\circ} 27^{\prime} 05^{\prime \prime}$ LONGITUDE : $66^{\circ} 21^{\prime} 35^{\prime \prime}$

DIAMETER : $16-18$ inches DEPTH : 160 feet

\begin{tabular}{|c|c|c|c|c|c|c|c|c|c|c|c|c|}
\hline \multirow[b]{2}{*}{ YEAR } & : & & & & \multicolumn{2}{|c|}{ Mol } & & & & & & \\
\hline & JAN & FEB & MAR & APR & MAY & JUN & JUL & AUG & SEP & OCT & NOV & DEC \\
\hline & ; & a & & a & & & & 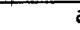 & & & & \\
\hline 1982 & 3.72 & 16.80 & 4.76 & 25.70 & 4.74 & 5.25 & 5.80 & 41.78 & 5.86 & 6.00 & 6.40 & 28.05 \\
\hline & : & & & $a$ & & & ${ }^{a}$ & $0^{\circ}$ & & & & \\
\hline 1983 & 6.25 & 6.70 & 6.91 & 33.10 & 6.60 & 6.85 & 34.50 & 35.80 & 6.25 & 6.30 & 6.32 & 6.48 \\
\hline 1984 & 6.80 & 6.68 & & & 7.39 & 7.03 & 6.96 & 6.88 & 6.62 & 6.40 & 5.89 & 6.03 \\
\hline
\end{tabular}

a pumping, $b$ recently pumped, e estimated, $h$ tape measurement, $j$ lowest water level recorded,

n nearby punping well 


\begin{tabular}{|c|c|c|c|c|c|c|c|c|c|c|c|c|}
\hline $\begin{array}{l}\text { LOCAL } \\
\text { WELL } \\
\text { LAT IT } \\
\text { DIAME }\end{array}$ & $\begin{array}{l}\text { NUMBER : } \\
\text { NAME : Ri } \\
\text { TUDE : } 1 B^{\circ} \\
\text { ETER : }\end{array}$ & $\begin{array}{l}168 \\
0 \text { Prog } \\
27 \cdot 51^{4} \\
16 \text { inch }\end{array}$ & an No. & $\begin{array}{l}\text { ELE } \\
\text { OWK } \\
\text { LON } \\
\text { DEP }\end{array}$ & $\begin{array}{l}\text { VATION } \\
\text { ER : AF } \\
\text { GITUDE } \\
\text { TH : } 15\end{array}$ & $\begin{array}{l}\text { ABOVE M } \\
\text { OA P.R. } \\
66^{\circ} 26 \\
\text { feet }\end{array}$ & $\begin{array}{l}\text { EAN SEA } \\
\text { Depart } \\
\text { '16" }\end{array}$ & $\begin{array}{l}\text { LEVEL: } \\
\text { ent of }\end{array}$ & $\begin{array}{l}\text { 9.B4 } \\
\text { Agric }\end{array}$ & $\begin{array}{l}\text { eet } \\
\text { iture }\end{array}$ & & \\
\hline YEAR & : & FEB & MAR & APR & MAY & $\begin{array}{l}\text { NTHS } \\
\text { JUN }\end{array}$ & JUL & AUG & SEP & $\mathrm{OCI}$ & NOV & $D E C$ \\
\hline & $-:$ & & & & & & $a$ & & & & a & a \\
\hline $19 B 2$ & $\vdots$ & & 6.15 & 6.30 & 6.60 & 6.80 & 15.60 & 6.67 & 6.55 & 6.50 & 13.68 & 13.45 \\
\hline & : & & & a & a & a & a & & & 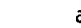 & a & \\
\hline 1983 & $: \quad 12.29$ & & 13.60 & 12.67 & 12.10 & 13.00 & 12.90 & 6.50 & 6.19 & 49.90 & $46.77^{\circ}$ & 43.90 \\
\hline & : & a & & & & & & & & 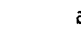 & & \\
\hline 1984 & 5.90 & 43.22 & & & 6.15 & 5.77 & 5.76 & 42.65 & 24.17 & 28.68 & 5.01 & 4.75 \\
\hline 1985 & 4.96 & & & & & & & & & & & \\
\hline
\end{tabular}

REMARKS: Well discontinued.

LOCAL NUMBER : 169 ELEVATION ABOVE MEAN SEA LEVEL: 13.10 feet

WELL NAME : Rice Program No. 5 OWNER : AFDA - P.R. Department of Agriculture

LATITUDE : $18^{\circ} 27^{\prime} 40^{\prime \prime}$

DIAMETER : 16 inches

DEPTH : 140 feet

\begin{tabular}{|c|c|c|c|c|c|c|c|c|c|c|c|c|}
\hline YEAR & JAN & FEB & MAR & APR & MAY ${ }^{\text {MO }}$ & $\begin{array}{c}\text { THS } \\
\text { JUN }\end{array}$ & JUL & AUG & SEP & OCT & NOV & DEC \\
\hline & $\vdots$ & & & & & & & & & & . & \\
\hline 1983 & : & & & & & & & & & 6.33 & 11.37 & 11.10 \\
\hline 1984 & $: 12.10^{\mathrm{a}}$ & $10.87^{a}$ & & & $1205^{\mathrm{a}}$ & 635 & $A 4$ & 737 & 62 & ${ }^{a}$ & 610 & 64 \\
\hline
\end{tabular}

REMARKS: Well discontinued.

a pumping, b recently pumped, e estimated, $h$ tape measurement, $j$ lowest water level recorded, $n$ nearby pumping well 


\section{WELLS IN THE \\ SOUTH COAST PROVINCE \\ Patilla to Ponce}

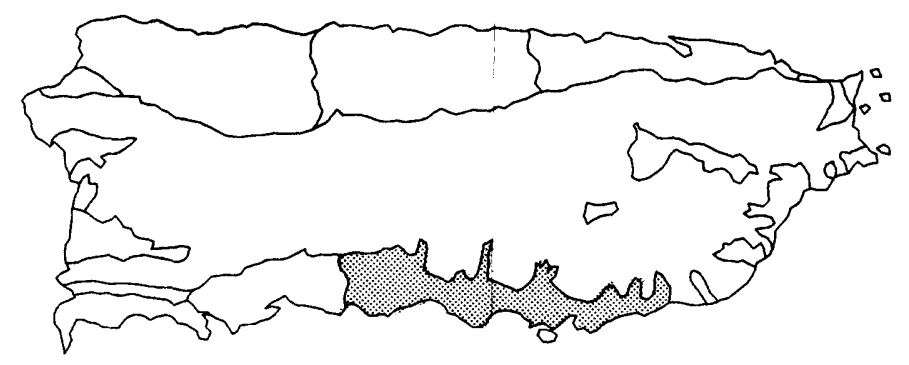

LOCAL NUMBER : 125

WELL NAME : Patillas, STP

LATITUDE : $10^{\circ} 00^{\prime} 10^{\prime \prime}$

ELEVATION ABOVE MEAN SEA LEVEL: 48 feet

OWMER : P.R. Aqueduct and Sewer Authority

LONGITUOE : $66^{\circ} 00^{\prime} 45^{\prime \prime}$

DIAMETER : $16-12$ inches

DEPTH : 90 feet

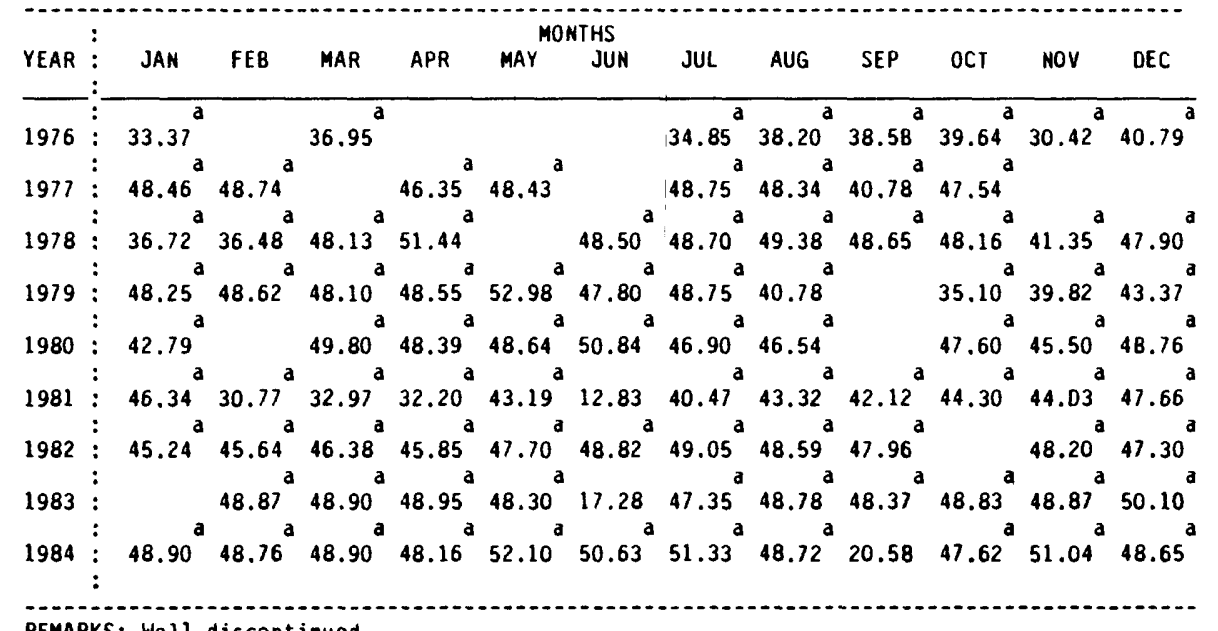

REMARKS: Well discont inued.

a punping, b recently punped, e estimated, $h$ tape measureaent, j lowest water level recorded, n nearby pumping well 


\begin{tabular}{|c|c|c|c|c|c|c|c|c|c|c|c|c|c|}
\hline $\begin{array}{l}\text { LOCAL } \\
\text { WELL } \\
\text { LAT IT } \\
\text { DIAME }\end{array}$ & $\begin{array}{l}\text { NI } \\
\text { NAI } \\
\text { UD } \\
\text { TE }\end{array}$ & $\begin{array}{l}\text { JMBER : } \\
\text { IE : Pa } \\
: 1 B^{\circ} \\
: 13\end{array}$ & $\begin{array}{l}7 \\
\text { llas } \\
18 \text { " } \\
\text { ches }\end{array}$ & 2 & $\begin{array}{l}\text { ELE } \\
\text { OWN } \\
\text { LON } \\
\text { DEP }\end{array}$ & $\begin{array}{l}\text { IATION } \\
R \text { : P. } \\
\text { ITUDE } \\
H: 10\end{array}$ & $\begin{array}{l}\text { BOVE } \\
\text { Aque } \\
66^{\circ} 01 \\
\text { feet }\end{array}$ & $\begin{array}{l}A N \text { SE } \\
\text { uct a } \\
32^{4}\end{array}$ & $\begin{array}{l}\text { LVEL: } \\
\text { Sewer }\end{array}$ & $\begin{array}{l}50 \mathrm{fe} \\
\text { Autho }\end{array}$ & & & \\
\hline & : & & & & & MO & & & & & & & \\
\hline YEAR & : & JAN & FEB & MAR & APR & MAY & JUN & JUL & AUG & SEP & $\mathrm{OCT}$ & NOV & $D E C$ \\
\hline 1959 & $:$ & & & & & & & & & 5.63 & 4.54 & 5.06 & 6.04 \\
\hline 1960 & : & 7.53 & 8.50 & 8.94 & 9.20 & 8.04 & 7.84 & 6.88 & 6.83 & 3.72 & 6.94 & 6.06 & 3.33 \\
\hline 1961 & $\vdots$ & 6.49 & 6.22 & 7.51 & 9.42 & 9.10 & 9.54 & 5.79 & 5.98 & 4.16 & 5.31 & 5.79 & 5.13 \\
\hline 1962 & $\vdots$ & 6.43 & 6.73 & 7.97 & 7.99 & 8.15 & 7.02 & 5.57 & 6.70 & 5.70 & 4.53 & 6.05 & 6.68 \\
\hline 1963 & : & 6.70 & 7.40 & 7.70 & 8.76 & 9.62 & 7.52 & 8.33 & & 6.68 & 7.66 & 7.73 & 7.68 \\
\hline 1964 & $:$ & 8.24 & 9.64 & 10.64 & 11.01 & 9.64 & 9.06 & 7.93 & 7.94 & 7.00 & 6.40 & 6.24 & 7.29 \\
\hline 1965 & : & 7.93 & 8.00 & B. 85 & 9.92 & 9.42 & 9.09 & 6.72 & 5.63 & 5.74 & $5.5 B$ & 5.81 & 5.85 \\
\hline 1966 & $\vdots$ & 6.57 & 6.69 & 8.64 & 8.51 & 9.19 & 7.66 & 7.43 & 6.23 & 6.41 & & 5.16 & 6.66 \\
\hline 1967 & $\vdots$ & 6.59 & 7.75 & 7.95 & 8.61 & 8.43 & 9.20 & 8.29 & 8.25 & 10.82 & 10.25 & 10.12 & 8.80 \\
\hline 1968 & $:$ & 9.58 & 8.50 & 10.04 & 11.60 & 10.70 & 5.10 & 8.00 & 6.30 & 6.50 & 8.39 & 9.40 & 8.28 \\
\hline 1969 & $\vdots$ & 10.39 & & & & & 7.03 & & 6.72 & 6.04 & & & 9.70 \\
\hline 1970 & : & 10.36 & & & & & 8.69 & & 9.22 & 8.22 & 9.01 & & 6.82 \\
\hline 1971 & $\vdots$ & 7.09 & 9.25 & 9.52 & 9.94 & & & 8.03 & 9.28 & 7.47 & 10.20 & & \\
\hline
\end{tabular}

REMARKS: Obstruction.

a pumping, b recently pumped, e estimated, $h$ tape measurement, $j$ lowest water level recorded, n nearby pumping well 


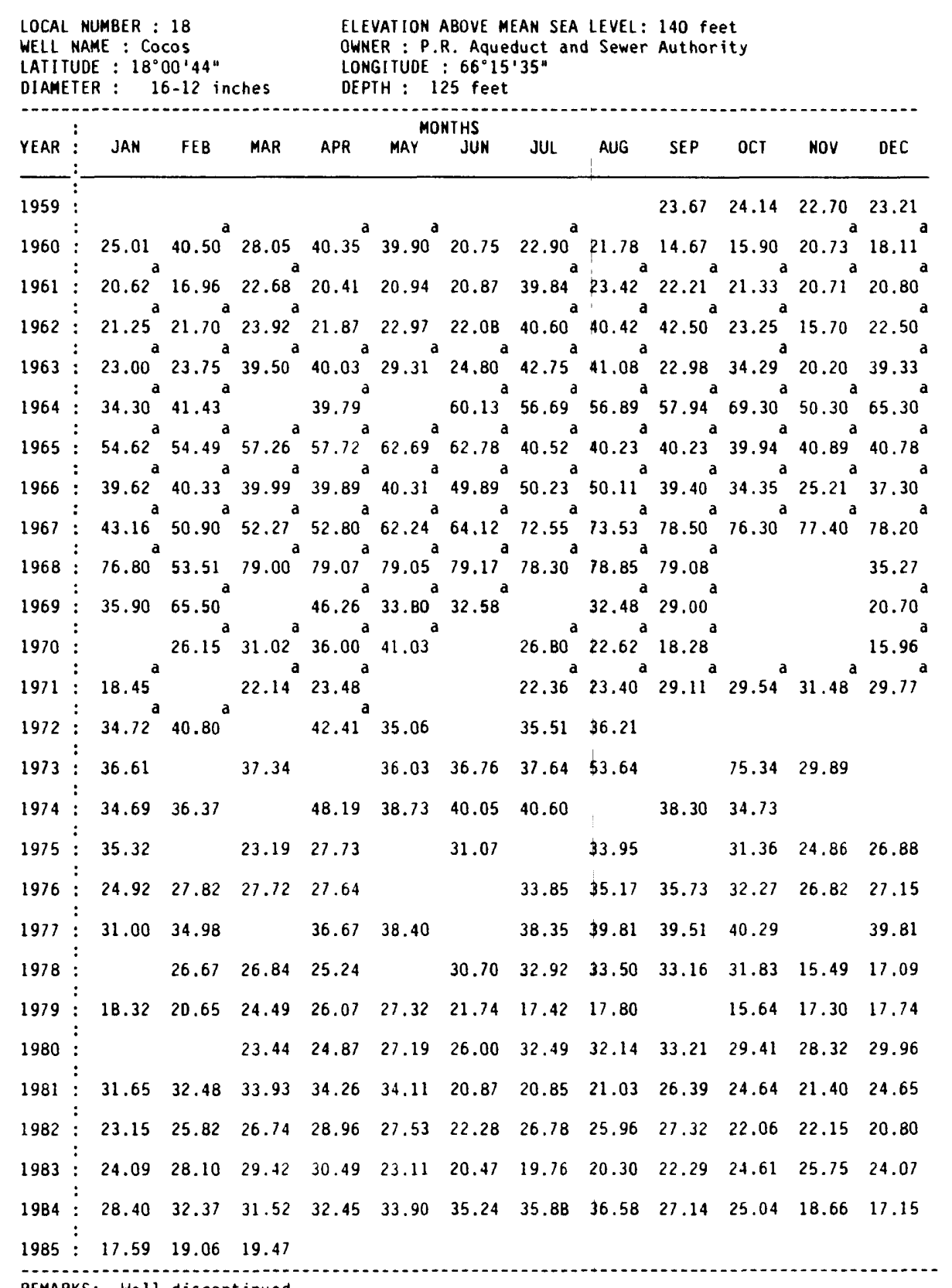

REMARKS: Well discont inued.

a pumping, b recently pumped, e estimated, $h$ tape measurenent, $j$ lowest water level recorded, $n$ nearby pumping well 


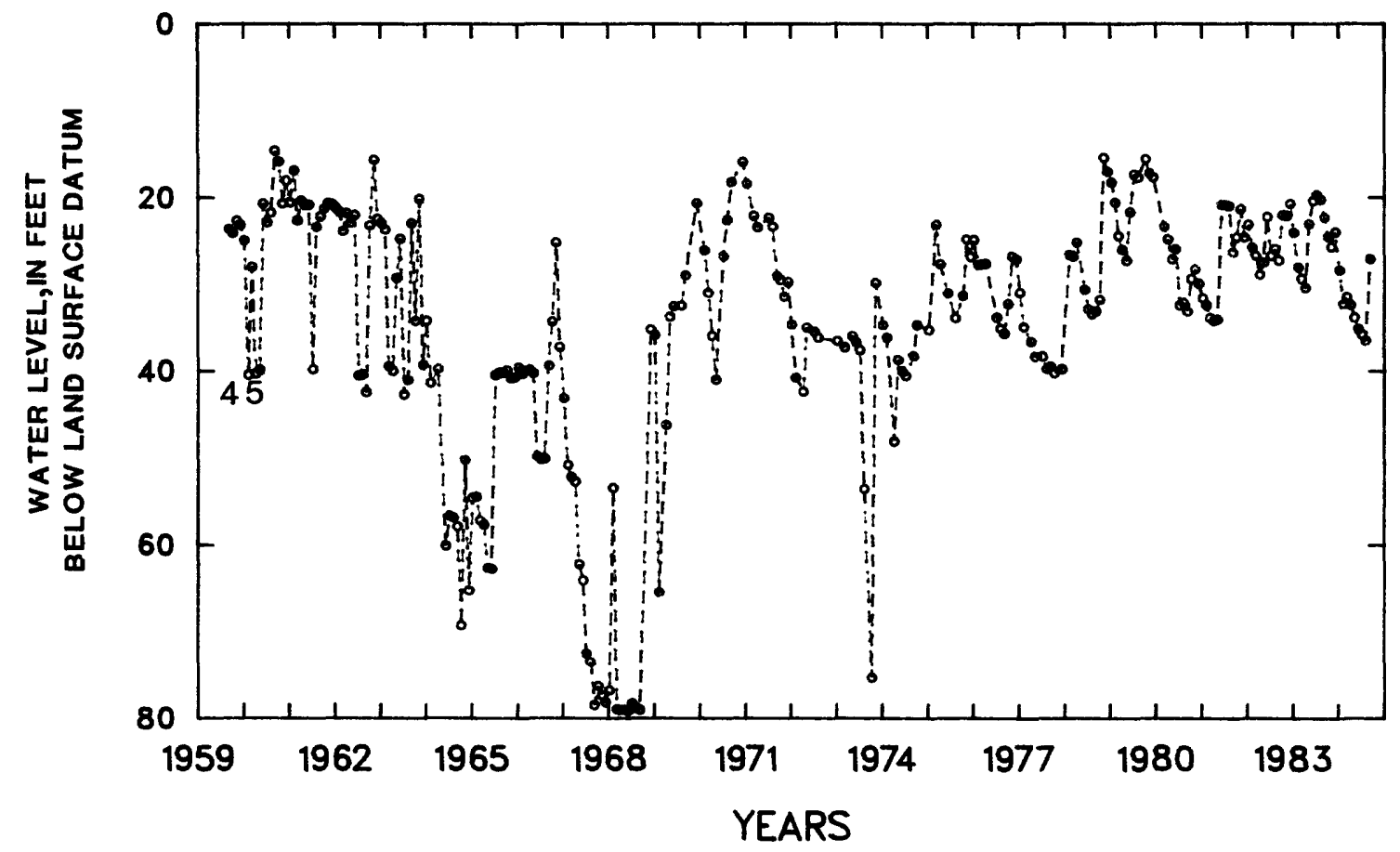

Figure 14.--Ground-water levels at Cocos, local number 18. 


\begin{tabular}{|c|c|c|c|c|c|c|c|c|c|c|c|c|c|}
\hline \multicolumn{5}{|c|}{$\begin{array}{l}\text { LOCAL NUMBER : } 19 \\
\text { WELL NAME : Theater no. } 1 \\
\text { LATITUDE : } 18^{\circ} 00^{\prime} 23^{\prime \prime} \\
\text { DIAMETER : } 11-16 \text { inches }\end{array}$} & $\begin{array}{l}\text { ELEV } \\
\text { OWNE } \\
\text { LONG } \\
\text { DEPT }\end{array}$ & $\begin{array}{l}\text { IATION } \\
E R: U . \\
\text { SI TUDE } \\
\text { IH : } 1\end{array}$ & $\begin{array}{l}\text { ABOVE M } \\
\text { S. Army } \\
: 66^{\circ} 17 \\
50 \text { feet }\end{array}$ & $\begin{array}{l}\text { EAN SEA } \\
\text { ' } 54^{\prime \prime}\end{array}$ & LEVEL: & $132 \mathrm{fe}$ & & & 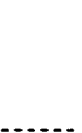 \\
\hline$\ldots$ & : & & & & 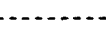 & MO & NTHS & & & & & & \\
\hline YEAR & : & JAN & FEB & MAR & APR & MAY & JUN & JUL & AUG & SEP & $O C T$ & NOV & DEC \\
\hline 1958 & : & & & & & & & & & & & & 55.12 \\
\hline 1959 & : & & & 56.40 & & & & & 57.28 & 54.91 & 56.89 & 56.66 & 57.14 \\
\hline 1960 & $\vdots$ & 57.44 & 57.62 & 58.13 & 58.25 & 58.37 & 58.52 & 58.35 & 57.97 & & 56.01 & 56.01 & 58.36 \\
\hline 1961 & $\vdots$ & 54.81 & 55.44 & 60.17 & 61.88 & 56.55 & 56.77 & 58.24 & 58.83 & 56.60 & 58.83 & 58.67 & 57.70 \\
\hline 1962 & $\vdots$ & 58.62 & 59.90 & 56.24 & 56.40 & 56.57 & 56.79 & & 52.75 & 56.70 & 57.10 & 55.00 & 55.13 \\
\hline 1963 & $\vdots$ & 55.58 & 55.85 & 56.40 & 56.30 & 56.15 & 56.20 & 56.36 & 56.19 & Obst & ruction & in the & we 11 \\
\hline 1964 & $:$ & from $s$ & ept ember & 1963 & to July & 1964. & & & 58.32 & 58.17 & 57.59 & 58.14 & 57.50 \\
\hline 1965 & $:$ & 58.05 & 58.12 & 59.85 & 60.31 & 61.29 & 61.34 & 63.73 & 63.08 & 57.28 & 57.14 & 58.89 & 58.77 \\
\hline 1966 & $\vdots$ & 58.07 & 61.99 & 62.11 & 63.23 & 63.40 & 59.80 & 59.99 & 58.50 & 58.88 & & 58.00 & 57.35 \\
\hline 1967 & $\vdots$ & 63.68 & 58.23 & 58.43 & 58.69 & 58.97 & 59.89 & 59.79 & 59.08 & 59.32 & 59.22 & 59.36 & 59.54 \\
\hline 1968 & $\vdots$ & & 60.50 & 59.60 & 59.42 & 59.62 & 59.02 & 58.31 & 58.10 & 57.65 & 56.89 & & 56.88 \\
\hline 1969 & $\vdots$ & 56.27 & 55.72 & & 58.37 & 57.80 & 58.34 & & 57.30 & 53.14 & & & 52.14 \\
\hline 1970 & $\vdots$ & & 52.52 & 52.90 & 56.09 & 54.05 & & 54.76 & 52.30 & 52.09 & & & 44.70 \\
\hline 1971 & : & 46.76 & & 50.79 & 52.99 & 52.07 & & 51.37 & 52.48 & 56.39 & 56.13 & 52.77 & 52.59 \\
\hline 1972 & : & 53.78 & 54.00 & & 53.79 & 54.05 & & 56.75 & 58.45 & & 54.33 & & \\
\hline 1973 & $\vdots$ & 60.55 & & 72.25 & 55.95 & 78.20 & 57.10 & & 78.77 & & 79.15 & & \\
\hline 1974 & $:$ & 55.84 & 56.51 & & 57.20 & $86+$ & $86+$ & $86+$ & & $86+$ & & $86+$ & \\
\hline 1975 & $:$ & 53.30 & & 55.71 & 58.11 & & 68.22 & & 72.22 & & 67.09 & 62.86 & 63.35 \\
\hline 1976 & : & 60.75 & 62.51 & 52.91 & 66.45 & & 55.45 & 54.56 & 54.80 & 52.26 & 50.94 & 49.68 & 51.45 \\
\hline 1977 & : & 52.48 & 56.01 & & 55.80 & 61.54 & & 61.57 & 54.90 & 58.63 & 56.72 & & 52.79 \\
\hline 1978 & $\vdots$ & & 57.23 & 58.65 & 53.44 & & 59.50 & 57.79 & 60.97 & 54.07 & 54.33 & 47.32 & 46.52 \\
\hline 1979 & $\vdots$ & 48.52 & 49.90 & 50.72 & 51.26 & 51.69 & 51.05 & 49.02 & 48.40 & & 39.38 & 39.89 & 40.54 \\
\hline 1980 & $\vdots$ & & & 48.70 & 49.44 & 50.48 & 51.39 & 51.57 & 51.95 & 50.78 & 51.08 & 51.66 & 52.44 \\
\hline 1981 & $\vdots$ & 54.78 & 53.94 & 53.80 & 53.44 & 57.34 & $51.8 B$ & 55.09 & 54.07 & 56.25 & 56.30 & 50.60 & 56.01 \\
\hline 1982 & $\vdots$ & 56.02 & 57.85 & 57.54 & 56.79 & 56.33 & 53.56 & 51.85 & 52.40 & 53.30 & 53.54 & 54.53 & 54.78 \\
\hline 1983 & : & 62.33 & 56.30 & 56.50 & 57.41 & 53.23 & 54.70 & 53.89 & 55.50 & 46.83 & 50.24 & 50.20 & 47.62 \\
\hline 1984 & $\vdots$ & 45.16 & 46.73 & 47.72 & 47.70 & 55.57 & 51.52 & 52.83 & 53.90 & 52.11 & 49.02 & 46.85 & 41.35 \\
\hline
\end{tabular}

Remarks: Depth 150 feet reported, 86 feet measured. We 11 discontinued.

a pumping, b recently pumped, e estimated, h tape measurement, $j$ lowest water 1 evel recorded, n nearby puaping well 


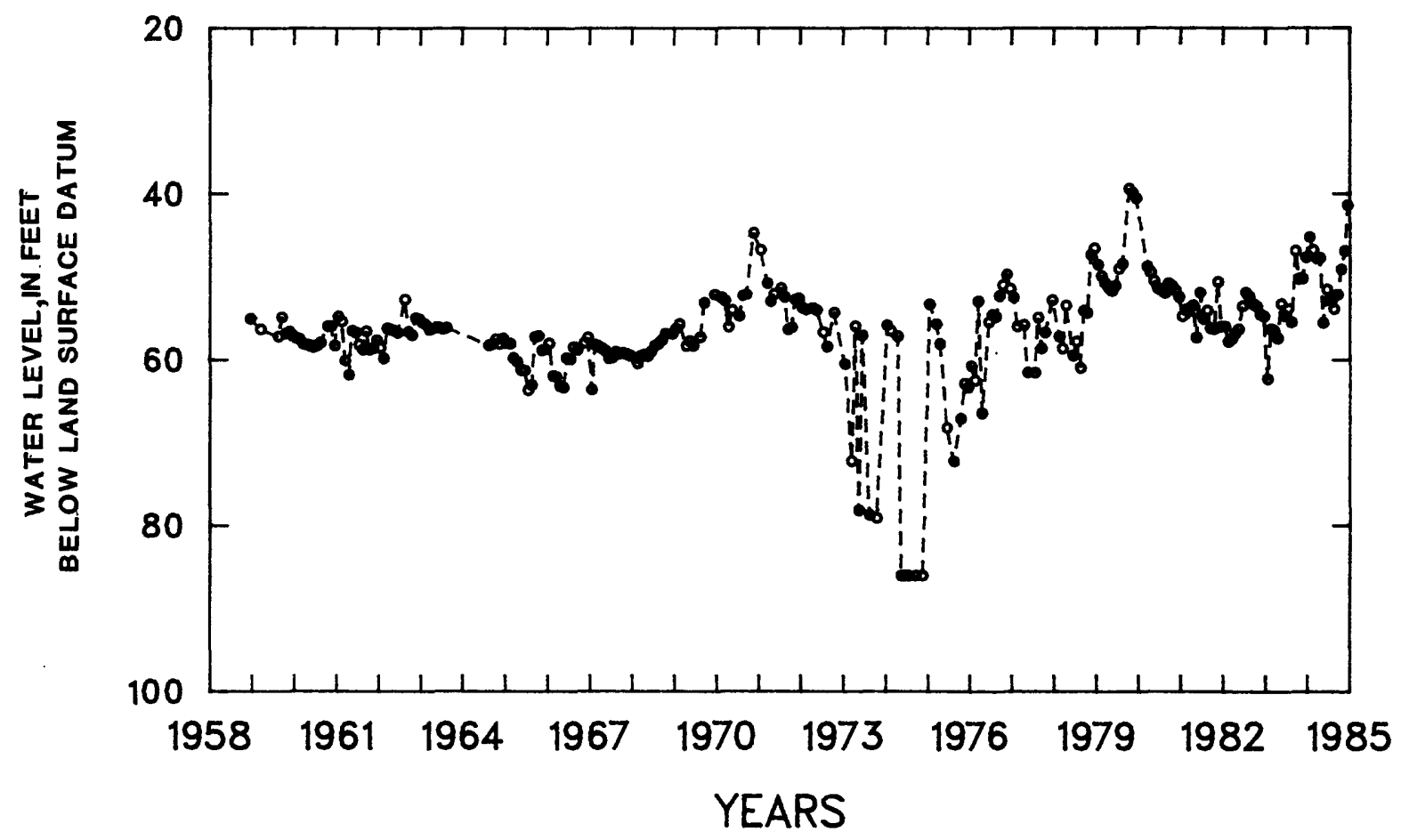

Figure 15.--Ground-water levels at Theater Number 1, local number 19. 
LOCAL MUMBER : 20

WELL MAME : $\mathrm{Ft}$. Allen no. 2

ELEVATION ABOVE MEAN SEA LEVEL: 22 feet

LAT ITUDE : $18^{\circ} 00^{\prime} 32^{\prime \prime}$

OWMER : U.S. ArR

LONGITUDE: $66^{\circ} 30^{\prime} 17^{\circ}$

DIAMETER : 12 inches DEPTH : 150 feet

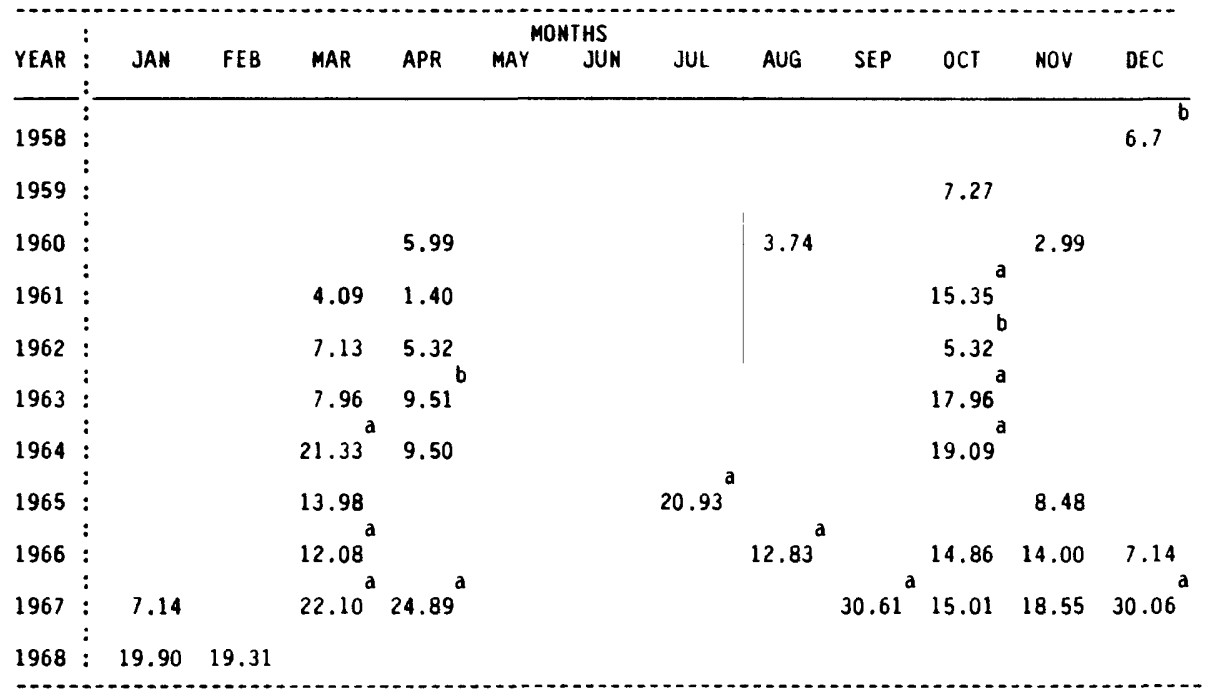

\begin{tabular}{|c|c|c|c|c|c|c|c|c|c|c|c|c|c|}
\hline $\begin{array}{l}\text { LOCAL } \\
\text { WELL } \\
\text { LAIII } \\
\text { DIAME }\end{array}$ & $\begin{array}{l}\text { N } \\
\text { NA } \\
\text { TUD } \\
\text { ETE }\end{array}$ & $\begin{array}{l}\text { JMBER : } \\
\text { ME : Ha } \\
\text { E : } 18^{\circ} \\
R: 19\end{array}$ & $\begin{array}{l}88 \\
\text { cienda } \\
0 \text { ' } 52 " \\
\text { inches }\end{array}$ & Potala & $\begin{array}{l}\text { ELEY } \\
\text { OWNE } \\
\text { LONE } \\
\text { DEPT }\end{array}$ & $\begin{array}{l}\text { VATION } \\
\text { ER : LU } \\
\text { GITUDE } \\
\text { TH : } 14\end{array}$ & $\begin{array}{l}\text { ABOVE M } \\
\text { ce and } \\
: 66^{\circ} 30 \\
3 \text { feet }\end{array}$ & $\begin{array}{l}\text { AN SEA } \\
\text { Ompany } \\
50 "\end{array}$ & EVE & $15 \mathrm{fe}$ & & & \\
\hline$\cdots$ & ${ }^{\circ}$ & & & & & MO & NTHS & & & & & & \\
\hline YEAR & : & JAN & FEB & MAR & APR & MAY & JUN & JUL & AUG & SEP & $\mathrm{OCT}$ & NOV & DEC \\
\hline 1968 & : & & & & & 36.50 & 37.74 & 37.89 & 37.24 & 35.48 & 31.22 & & 14.34 \\
\hline 1969 & $\vdots$ & 18.02 & & 16.19 & 23.38 & 12.30 & & 13.27 & 11.16 & 19.17 & & & 12.29 \\
\hline 1970 & $\vdots$ & & 20.58 & 16.98 & 22.94 & 14.33 & & 10.69 & 11.38 & 9.70 & 4.50 & & 13.12 \\
\hline 1971 & $\vdots$ & 10.50 & & & & 10.87 & & 13.19 & 9.88 & & & & 13.67 \\
\hline 1972 & : & 14.90 & 17.17 & & 16.55 & 16.28 & & & 27.57 & & & 21.14 & \\
\hline 1973 & $\vdots$ & 20.17 & & & & & & & & & & & \\
\hline $\begin{array}{l}1974 \\
1975\end{array}$ & $\begin{array}{l}: \\
\vdots\end{array}$ & & & & & & & & & & & & \\
\hline 1976 & $\vdots$ & & & & $28.76^{a}$ & $30.33^{a}$ & $31.08^{a}$ & & & & 36.09 & 30.95 & 29.19 \\
\hline 1977 & : & 29.56 & 31.33 & & 34.12 & 36.70 & $35.4 B$ & 35.12 & 31.19 & 28.52 & 27.88 & & \\
\hline 1978 & $\vdots$ & 20.45 & 19.52 & 18.95 & 19.56 & 21.36 & 23.88 & 23.56 & 24.26 & 24.98 & 25.52 & 18.95 & 18.01 \\
\hline 1979 & $:$ & 17.88 & 18.29 & 20.86 & 31.77 & 25.13 & 22.88 & 19.22 & 15.87 & & 12.78 & 14.82 & 14.66 \\
\hline $\begin{array}{l}1980 \\
1981\end{array}$ & $\begin{array}{l}\vdots \\
\vdots \\
\vdots\end{array}$ & 15.62 & 15.78 & & & 14.24 & 13.52 & 11.10 & 7.56 & 18.52 & & $n$ & \\
\hline 1982 & : & $\begin{array}{r}19.36 \\
n\end{array}$ & 14.37 & & 24.48 & 17.95 & 21.71 & 25.11 & 17.37 & 16.89 & 16.51 & 28.24 & 17.55 \\
\hline 1983 & $\vdots$ & 27.00 & $2 B .37$ & 20.62 & 28.14 & 17.30 & 14.17 & 14.28 & 21.25 & 22.72 & 17.00 & 15.60 & 13.35 \\
\hline $\begin{array}{l}1984 \\
1985\end{array}$ & : & $\begin{array}{l}21.23^{n} \\
24.17^{n}\end{array}$ & $\begin{array}{c}15.44^{n} \\
23.55^{n}\end{array}$ & $\begin{array}{c}16.68 \\
18.52^{n}\end{array}$ & 17.06 & 18.49 & 27.18 & 21.20 & 28.80 & 21.43 & 18.91 & 22.02 & 14.17 \\
\hline
\end{tabular}

REMARKS: Well discontinued.

a pumping, b recently pumped, estimated, $h$ tape measurement, $j$ lowest water level recorded, n nearby pumping well 


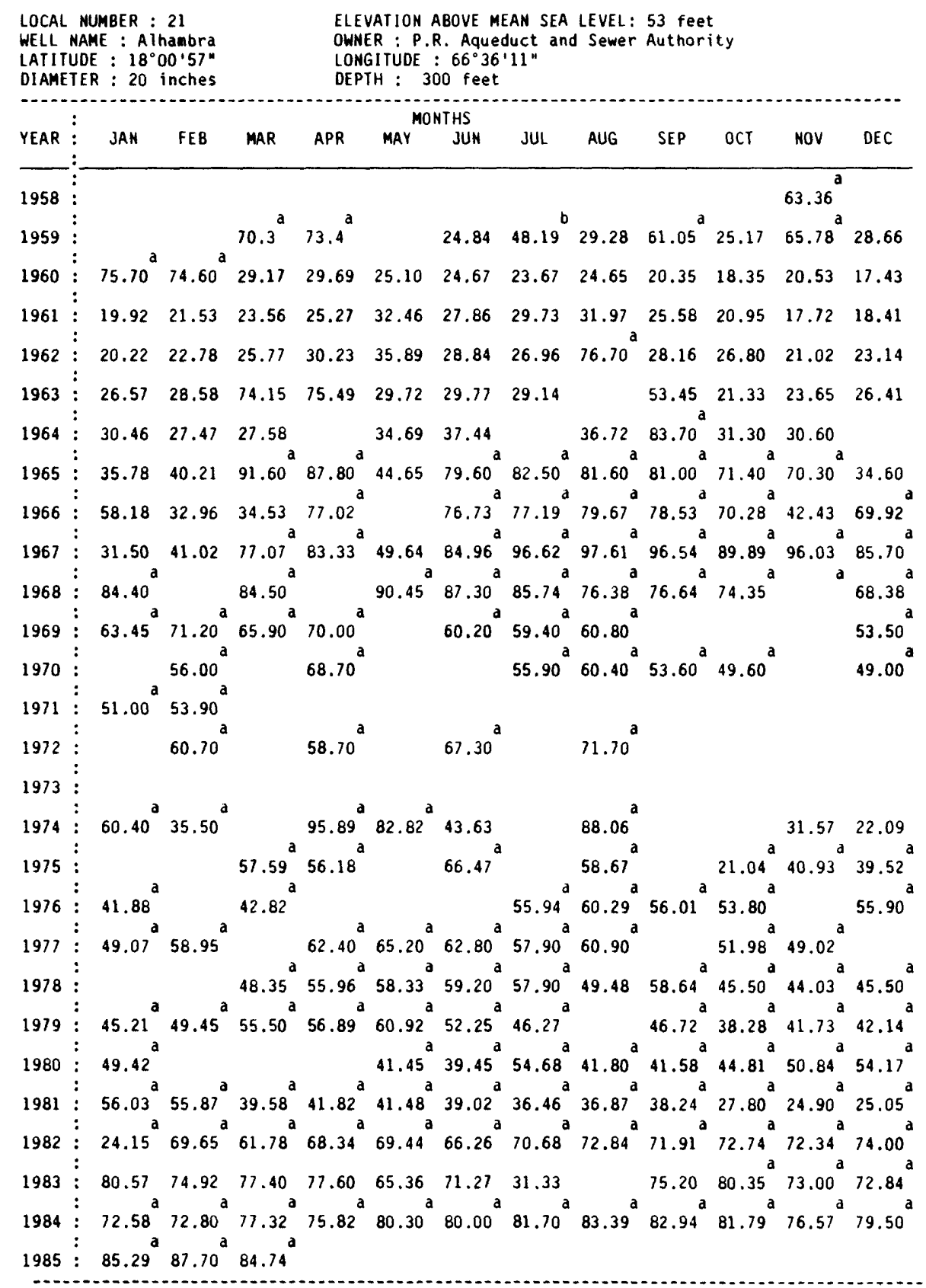

REMARKS: Well discontinued.

a pumping, b recently pumped, e est imated, $h$ tape measurement, $j$ lowest water level recorded, n nearby pumping well 


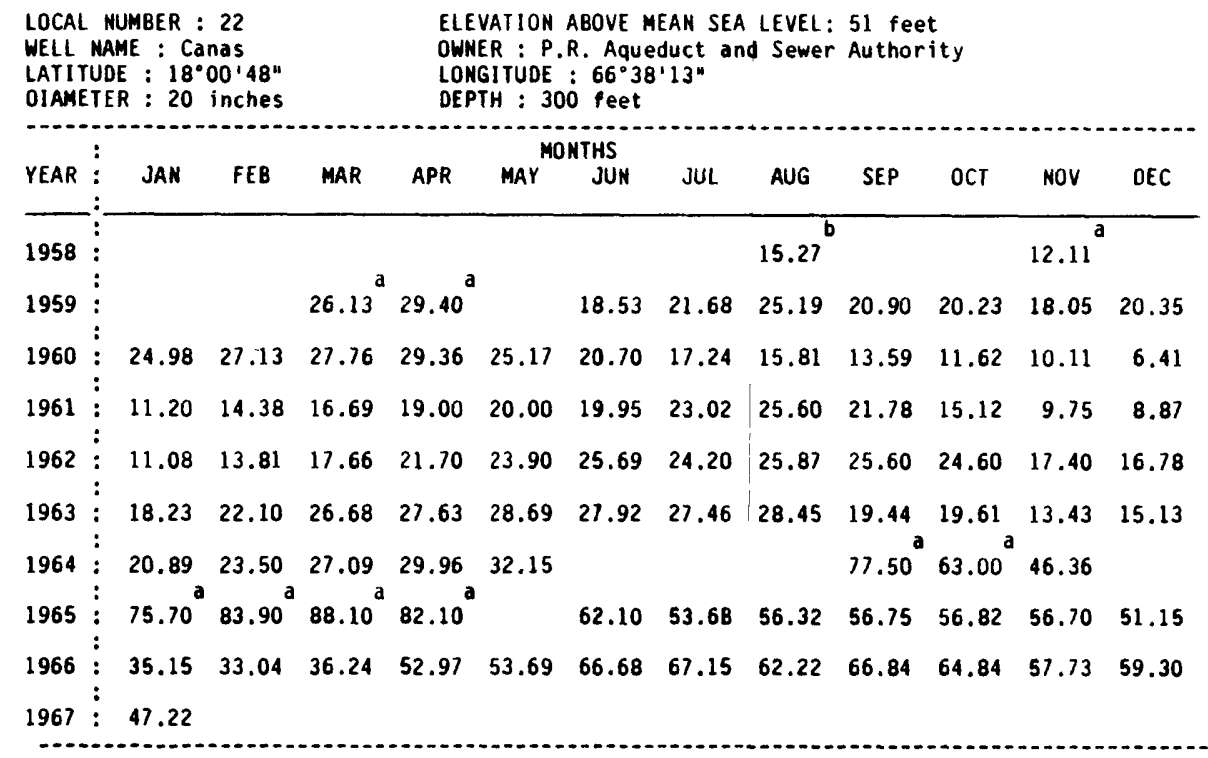

REMARKS: Well discont inued.

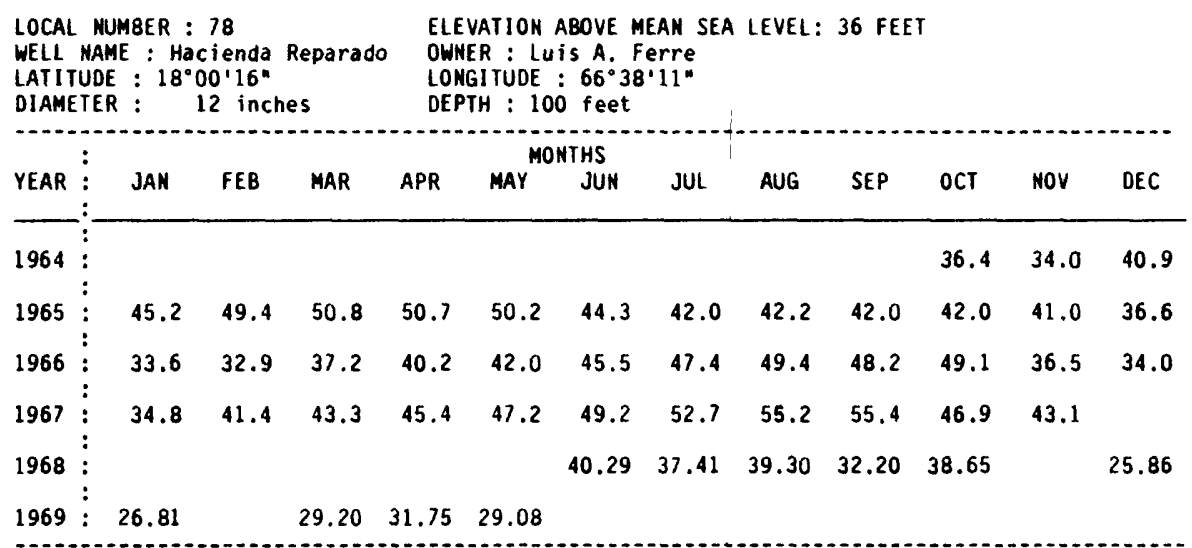

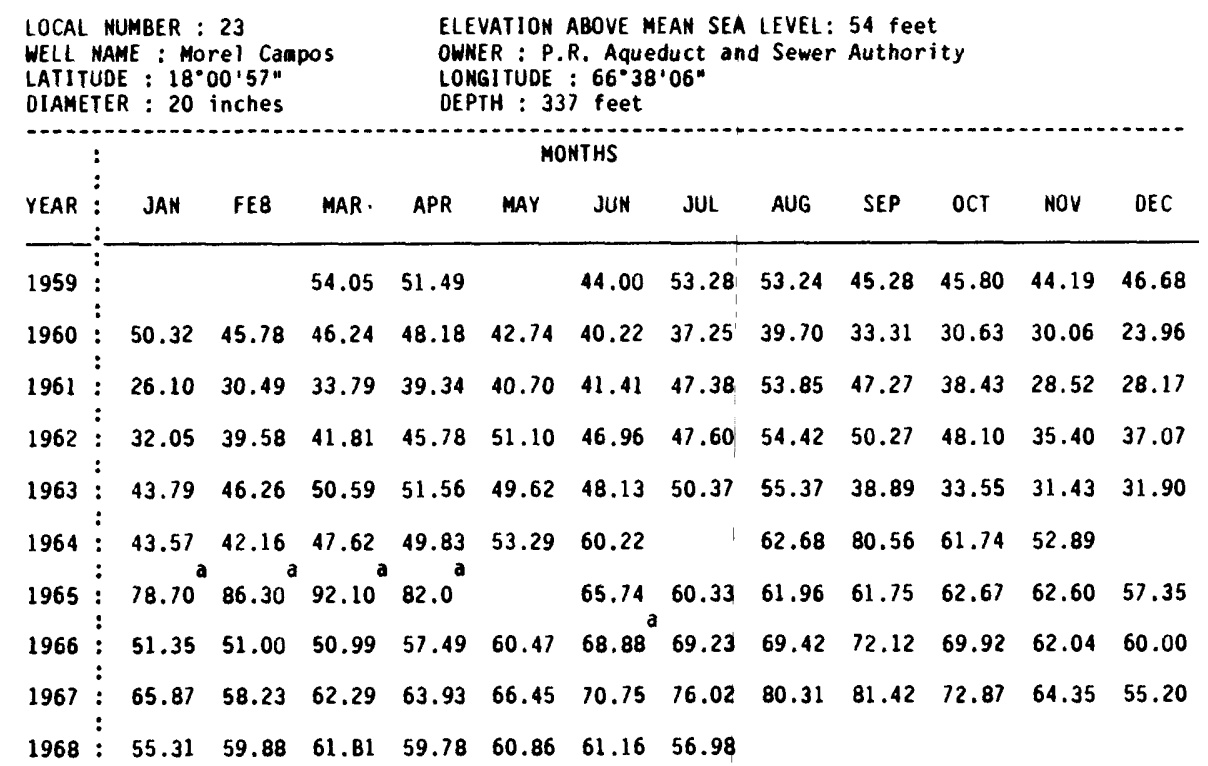

Rearks: Station discontinued $08 / 1968$. Well discontinued.

a pumping, b recently pumped, e estimated, $h$ tape measurement, $j$ lowest water level recorded, n nearby pumping well 


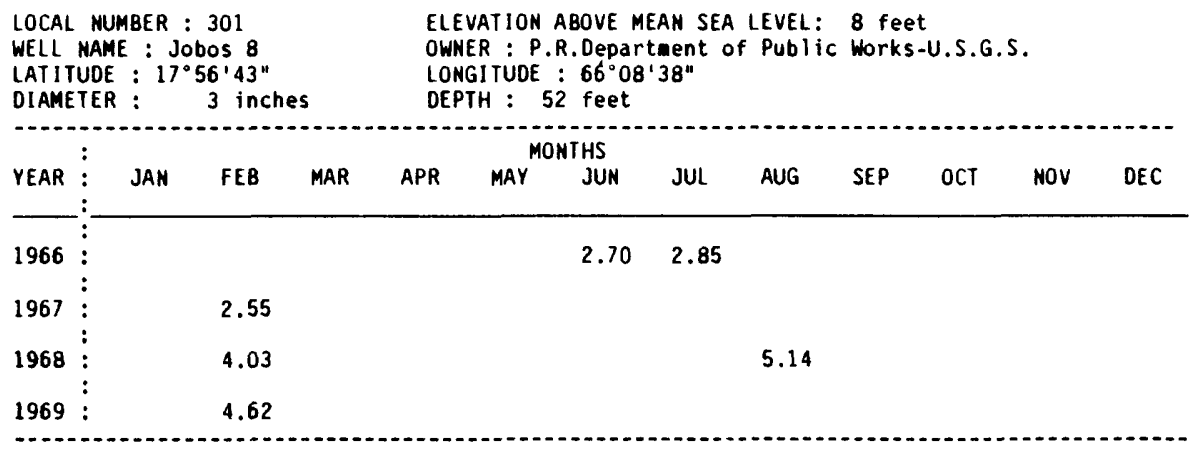

LOCAL NUMBER : 89 ELEVATION ABOVE MEAN SEA LEVEL: 6 feet

WeLl NAME : Phillips Obs. Well 3 OWNER : Phillips P.R. Core, Inc.

LATITUDE : $17^{\circ} 56^{\prime} 40^{\prime \prime}$ LONGITUDE: $66^{\circ} 08^{\prime} 51^{\prime \prime}$

DIAMETER : 4 inches DEPTH : 114 feet

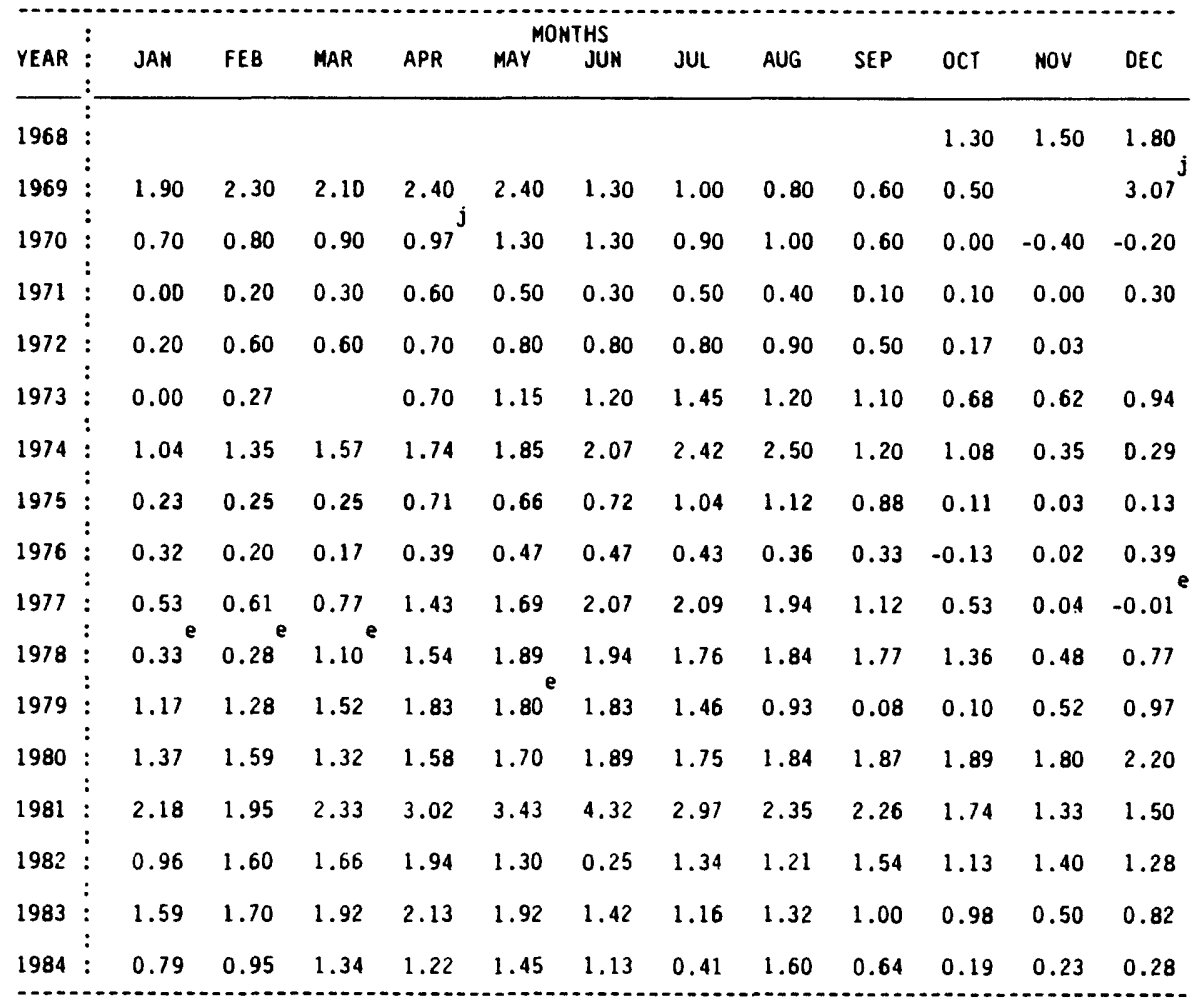

REMARKS: Well discontinued: Changed to a partial site since October 1,1981

a pumping, b recently pumped, e estimated, $h$ tape measurement, $j$ lowest water level recorded,

n nearby punping wel 
LOCAL NUMBER : 302

WELL MAME : Jobos 3

LATITUDE : $17^{\circ} 56^{\prime} 45^{\prime \prime}$

DIAMETER : 3 inches

ELEVATION ABOVE MEAN SEA LEVEL: 3 feet

OWNER : LUCE and CO., USGS

ONGITUDE : $66^{\circ} 09^{\prime} 23^{\prime \prime}$

DEPTH : 110 feet

\begin{tabular}{|c|c|c|c|c|c|c|c|c|c|c|c|c|}
\hline & : & & & & & & & & & & & \\
\hline YEAR & : JAN & FEB & MAR & APR & MAY & JUN & JUL & AUG & SEP & DCT & NOV & DEC \\
\hline 1966 & : & & & & & -0.35 & -0.50 & & & & & \\
\hline 1967 & : & 0.95 & & & & & & & & & & \\
\hline 1968 & $:$ & -1.62 & & & & & & -3.56 & & & & \\
\hline 1969 & : & & & & & & & & & & & \\
\hline 1970 & : & 0.85 & & & & & & 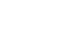 & & & & \\
\hline & : & & & & & & & & & & & \\
\hline 1971 & : & 0.30 & & & & & & & & & & \\
\hline 1972 & $:$ & -1.67 & & & & & & & & & & \\
\hline
\end{tabular}

LOCAL MUMBER : 1

ELEVATION ABOVE MEAN SEA LEVEL: 3 feet

WELL MAME : Mar Megro OWMER : P.R. Aqueduct and Sewer Authority.

LATITUDE : $17^{\circ} 56^{\prime} 58^{\prime \prime}$

LONGITUDE : $66^{\circ} 15^{\prime} 53^{\circ}$

DIAMETER : 3 inches DEPTH : 23 feet

\begin{tabular}{|c|c|c|c|c|c|c|c|c|c|c|c|c|c|}
\hline \multirow{3}{*}{$\begin{array}{l}\text { YEAR } \\
1959\end{array}$} & : & \multirow[b]{2}{*}{ JAN } & \multirow[b]{2}{*}{ FEB } & \multirow[b]{2}{*}{ MAR } & \multirow[b]{2}{*}{ APR } & \multicolumn{2}{|c|}{ MONTHS } & \multirow[b]{2}{*}{ JUL } & \multirow[b]{2}{*}{ AUG } & \multirow[b]{2}{*}{ SEP } & \multirow[b]{2}{*}{ OCT } & \multirow[b]{2}{*}{ MOV } & \multirow[b]{2}{*}{ DEC } \\
\hline & : & & & & & MAY & JUN & & & & & & \\
\hline & : & & & & & & & & & 0.38 & -0.75 & 0.05 & -0.75 \\
\hline 1960 & : & 0.26 & -0.21 & -0.51 & 0.45 & 0.55 & 0.71 & 0.39 & 0.44 & -0.56 & -0.50 & -0.64 & -0.75 \\
\hline 1961 & : & -0.43 & -0.69 & -0.60 & -0.27 & -0.75 & -0.75 & -0.75 & -0.30 & -0.41 & -0.75 & -0.75 & -0.75 \\
\hline 1962 & : & -0.75 & -0.62 & -0.57 & -0.75 & -0.51 & -0.10 & -0.27 & -0.02 & -0.75 & -0.24 & -0.49 & -0.55 \\
\hline 1963 & : & -0.73 & -0.38 & -0.45 & -0.75 & -0.19 & -0.05 & -0.10 & 0.28 & -0.75 & -0.71 & -0.52 & -0.29 \\
\hline 1964 & : & -0.75 & -0.18 & 0.09 & -0.04 & 0.82 & 0.65 & 0.93 & 1.07 & 1.25 & -0.65 & 0.02 & 0.66 \\
\hline 1965 & : & 1.15 & 1.06 & 2.00 & 2.10 & 1.88 & 1.94 & 1.79 & 1.44 & 1.75 & 1.84 & 1.52 & 1.73 \\
\hline 1966 & : & 0.97 & 1.02 & 0.69 & 0.78 & 0.96 & 0.42 & 0.35 & 0.27 & 1.03 & 1.24 & -0.75 & -0.21 \\
\hline 1967 & : & 0.95 & 0.67 & 0.91 & 1.23 & 1.01 & 2.89 & 1.84 & 1.62 & 1.81 & 1.67 & 1.87 & 1.64 \\
\hline 1968 & : & 1.17 & 2.15 & 2.77 & 2.52 & 2.95 & 3.15 & 2.69 & 2.66 & 1.22 & 1.57 & 1.34 & 1.89 \\
\hline 1969 & $\vdots$ & 2.10 & 2.26 & & 1.03 & 0.70 & 0.61 & & 0.67 & 1.45 & & & 0.13 \\
\hline 1970 & : & & 0.67 & 1.17 & 0.33 & 1.34 & & 0.55 & 0.14 & -0.36 & & & -1.83 \\
\hline 1971 & : & -1.30 & -0.96 & -1.64 & -0.97 & -1.20 & & 0.51 & 0.80 & 0.02 & & -1.67 & -1.53 \\
\hline 1972 & $\vdots$ & -0.71 & -0.04 & & 0.75 & 0.43 & & 1.56 & 0.84 & & 1.64 & 1.17 & \\
\hline 1973 & $:$ & 1.07 & & 1.17 & 0.89 & 1.36 & 1.34 & 1.57 & 0.85 & & 1.42 & 0.37 & \\
\hline 1974 & : & 0.98 & -0.44 & & 1.64 & 1.69 & $1.8 \mathrm{~B}$ & 3.11 & & 0.47 & 0.30 & -0.59 & 1.69 \\
\hline 1975 & : & 1.70 & & 1.00 & 1.19 & & 1.70 & & 1.11 & & 0.60 & 1.68 & 1.30 \\
\hline 1976 & : & 1.23 & 1.74 & 2.13 & 2.41 & & & 2.00 & 1.73 & 1.67 & 0.71 & 1.44 & 2.47 \\
\hline 1977 & : & 2.13 & 1.66 & & 2.36 & 1.79 & & 3.60 & 1.92 & 1.67 & 0.78 & & 0.78 \\
\hline 1978 & : & 1.07 & 1.84 & 2.54 & 1.25 & & 2.35 & 1.83 & 2.53 & 1.68 & 1.68 & -0.15 & 1.15 \\
\hline 1979 & : & 1.13 & 0.90 & 1.46 & 1.12 & 1.02 & 1.03 & 0.37 & 0.71 & & -0.95 & -1.47 & -0.40 \\
\hline 1980 & : & & & -0.04 & & 0.24 & 0.02 & 0.43 & 0.75 & 1.31 & 0.89 & 1.45 & 1.40 \\
\hline 1981 & : & 1.90 & 1.43 & 2.29 & 2.38 & 1.77 & 0.40 & 1.91 & 1.20 & 1.97 & 1.90 & 0.55 & 1.40 \\
\hline 1982 & : & 0.63 & 0.53 & 1.84 & 1.35 & 1.19 & 0.91 & 0.69 & 0.23 & 0.38 & 0.19 & 0.51 & 0.33 \\
\hline 1983 & : & 0.69 & 0.80 & 0.39 & 0.71 & 0.00 & 0.30 & -0.22 & 0.14 & -0.03 & -0.20 & -0.11 & -0.12 \\
\hline 1984 & : & -0.31 & -0.36 & 0.30 & 0.69 & 1.05 & 0.03 & -0.05 & 0.31 & -0.78 & -2.12 & -0.65 & -0.97 \\
\hline
\end{tabular}




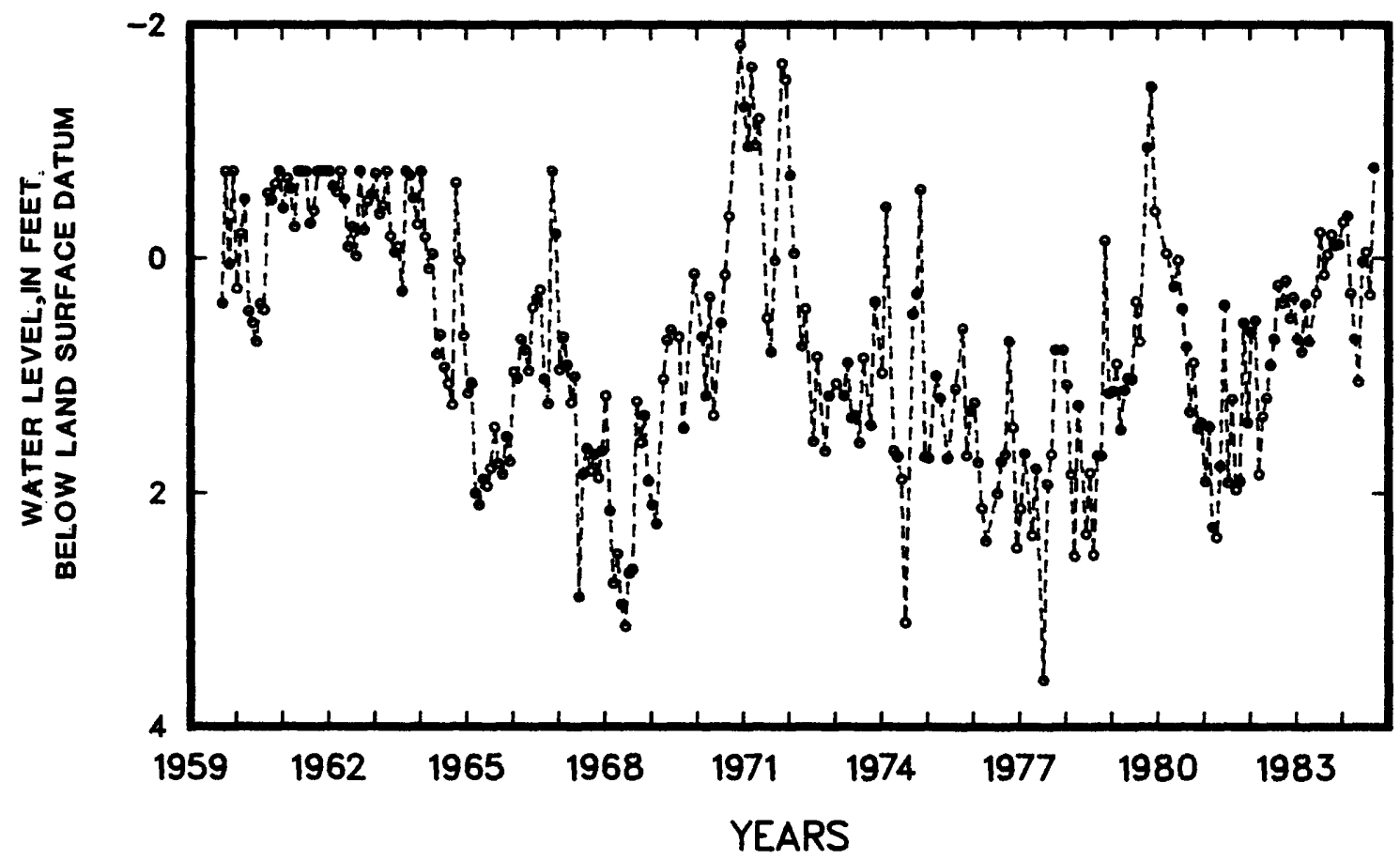

Figure 16.--Ground-water levels at Mar Negro, local number 1. 


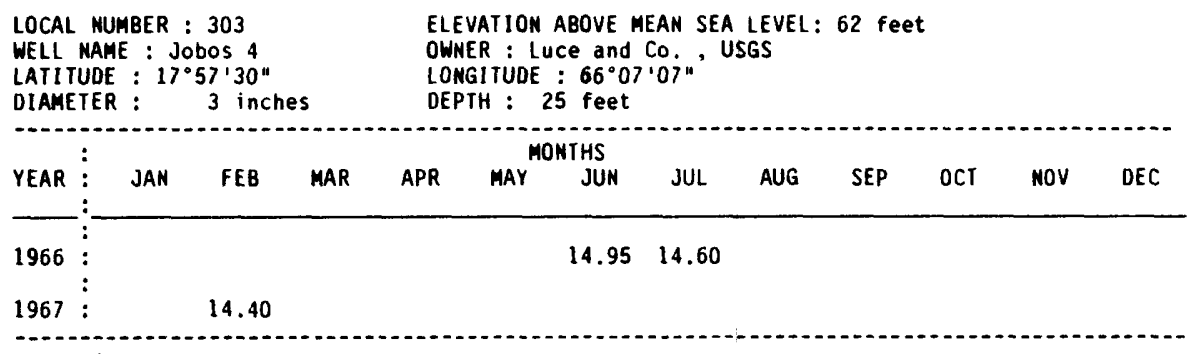

\begin{tabular}{|c|c|c|c|c|c|c|c|c|c|c|c|c|}
\hline $\begin{array}{l}\text { LOCAL } \\
\text { WELL } \\
\text { LATIT } \\
\text { DIAME }\end{array}$ & $\begin{array}{l}\text { MUMBER : } \\
\text { NAME : Me } \\
\text { UDE : } 17^{\circ} \\
\text { TER : } 16\end{array}$ & $\begin{array}{l}80 \\
\text { lania } \\
57 \text { '55" } \\
0 \text { inch }\end{array}$ & & $\begin{array}{l}\text { ELE } \\
\text { OWN } \\
\text { LON } \\
\text { DEP }\end{array}$ & $\begin{array}{l}\text { IATION } \\
\text { ER : Ce } \\
\text { II TUDE } \\
\text { IH : } 10\end{array}$ & $\begin{array}{l}\text { ABOve } \\
\text { tral A } \\
66^{\circ} 08 \\
\text { feet }\end{array}$ & $\begin{array}{l}\text { EAN SEA } \\
\text { gu irre } \\
4 B^{\prime \prime}\end{array}$ & LEVEL: & $60 \mathrm{fe}$ & & & \\
\hline - & : & & & & MO & TTHS & & & & & & \\
\hline YEAR & JAN & FEB & MAR & APR & MAY & JUN & JUL & AUG & SEP & $\mathrm{OCT}$ & HOV & DEC \\
\hline 1961 & : & & & & & & & & & & 10.80 & $11: 39$ \\
\hline 1962 & $: 12.75$ & & & & & & & & & & & \\
\hline 1963 & $\begin{array}{l}\text { : } \\
\text { : }\end{array}$ & & & & & & & & & & & 20.39 \\
\hline 1964 & $\vdots$ & & 25.04 & & & & & & & & & \\
\hline 1965 & $\vdots$ & & 25.95 & 26.50 & 26.70 & 26.55 & 22.20 & 22.10 & 19.80 & 19.00 & & 12.30 \\
\hline 1966 & $: 17.15$ & 18.00 & 18.00 & & 18.50 & & & & & & & \\
\hline
\end{tabular}

REMARK: Depth 119 feet reported, 103 feet measured. Well discont inued May, 1966.

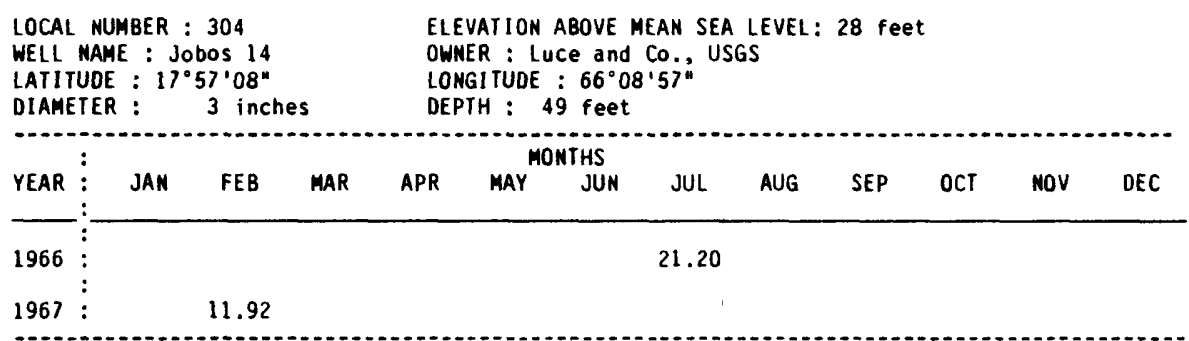

a pumping, b recently pumped, e estimated, $h$ tape measurement, $j$ lowest water level recorded, n nearby pumping well 


\begin{tabular}{|c|c|c|c|c|c|c|c|c|c|c|c|c|c|}
\hline $\begin{array}{l}\text { LOCAL } \\
\text { WELL } \\
\text { LATIT } \\
\text { DIAME }\end{array}$ & & $\begin{array}{l}\text { MBER : } \\
\begin{array}{l}: \text { Pu } \\
: 17^{\circ} \\
: 12\end{array}\end{array}$ & $\begin{array}{l}2 \\
\text { ente Jot } \\
57 \cdot 35 " \\
\text { inches }\end{array}$ & & $\begin{array}{l}\text { ELEI } \\
\text { OWNE } \\
\text { LONC } \\
\text { DEPT }\end{array}$ & $\begin{array}{l}\text { VATION } \\
\text { ER : P. } \\
\text { GITUDE } \\
\text { TH : } 14\end{array}$ & $\begin{array}{l}\text { ABOVE } \\
\text { R. Aque } \\
: 66^{\circ} 09 \\
8 \text { feet }\end{array}$ & $\begin{array}{l}\text { EAN SEA } \\
\text { duct an } \\
\text { ' } 59^{\prime \prime}\end{array}$ & $\begin{array}{l}\text { LEVEL: } \\
\text { d Sewer }\end{array}$ & $\begin{array}{l}26 \mathrm{fe} \\
\text { Autho }\end{array}$ & & & \\
\hline & : & & & & & & NTHS & & & & & & \\
\hline YEAR & : & JAN & FEB & MAR & APR & MAY & JUN & JUL & AUG & SEP & $\mathrm{OCT}$ & NOV & DEC \\
\hline & & & $a$ & a & a & a & a & $a$ & a & $a$ & $\bar{a}$ & a & $\bar{a}$ \\
\hline 1961 & : & & 55.80 & $5 B .22$ & 57.06 & 55.30 & 56.06 & 57.44 & 57.51 & 57.62 & 46.19 & 44.30 & 57.30 \\
\hline & : & $\mathbf{a}$ & a & & & a & a & a & $\mathbf{a}$ & $a$ & a & $\mathbf{a}$ & $\mathbf{a}$ \\
\hline 1962 & : & 56.30 & 49.84 & 55.33 & 4.55 & 54.79 & 55.50 & 56.30 & 55.30 & $56.30^{\circ}$ & $58.30^{\circ}$ & 55.07 & 54.77 \\
\hline & : & & & & & a & a & a & a & a & a & a & a \\
\hline 1963 & : & $56.5 B$ & 57.01 & 57.55 & 57.83 & 56.75 & 57.30 & 57.23 & 60.25 & 56.61 & 57.08 & 56.78 & 56.92 \\
\hline 1964 & : & $55.18^{a}$ & $58.69^{\mathrm{a}}$ & $5 B .65^{a}$ & & $57.60^{a}$ & $59.6 \mathrm{~B}^{\mathrm{a}}$ & $59.69^{a}$ & $61.78^{a}$ & $54.40^{a}$ & $61.36^{\mathrm{a}}$ & $60.20^{a}$ & $59.95^{\mathrm{a}}$ \\
\hline & $:$ & a & a & & & & & & & & & & \\
\hline 1965 & : & 59.16 & 59.04 & 58.22 & 16.08 & 14.56 & 14.93 & 14.72 & 13.07 & 55.05 & 54.77 & 11.85 & 12.18 \\
\hline & : & (a) & & & & a & & & & & & & \\
\hline 1966 & : & 54.62 & 49.52 & 51.37 & 51.34 & 51.83 & 13.52 & 14.22 & 13.99 & 13.32 & 11.76 & 10.21 & 9.97 \\
\hline 1967 & $:$ & 10.57 & 11.35 & 11.88 & 12.68 & 12.94 & 14.76 & 15.24 & 16.48 & $60.35^{a}$ & $59.20^{\mathrm{a}}$ & $55.90^{a}$ & $59.39^{\mathrm{a}}$ \\
\hline & : & & & & & & $a^{a}$ & $e^{a}$ & a & a & a & a & a \\
\hline 1968 & : & 56.43 & 60.55 & 58.55 & 58.39 & 57.77 & 59.90 & 57.95 & 58.78 & 59.28 & 58.20 & 57.80 & 57.86 \\
\hline & : & & & & (0) & $5667^{\mathrm{d}}$ & $654^{a}$ & & 5) ${ }^{a}$ & 5) $20^{a}$ & & & 57) \\
\hline 1969 & : & 59.60 & 60.12 & & 59.38 & 56.67 & 55.84 & & 57.45 & 57.28 & & & 57.40 \\
\hline 1970 & $\vdots$ & $56.76^{\circ}$ & & $59.92^{\circ}$ & 60.67 & 11.95 & 11.14 & & 10.25 & 8.91 & 7.74 & 5.53 & 5.90 \\
\hline 1971 & : & 5 & 19 & 6.21 & 5.12 & 353 & 5.51 & & 3.07 & 4,43 & 456 & & 5 \\
\hline & $\vdots$ & 200 & 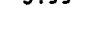 & 0.28 & & & & & 0.06 & 7.00 & 7.00 & & (1. \\
\hline 1972 & : & 5.35 & 6.17 & & 6.41 & 8.00 & & 3.32 & 9.09 & & & & \\
\hline 1973 & : & & & & & 9.50 & 9.25 & 10.23 & 8.85 & & 6.70 & 6.95 & \\
\hline 1974 & $\vdots$ & 7.34 & 7.52 & & 8.72 & 9.92 & 10.12 & 11.84 & & 10.30 & 5.56 & & 5.15 \\
\hline 1975 & : & 5.10 & 5.57 & 4.94 & 5.59 & & 6.17 & & 5.94 & & 5.15 & 5.94 & 5.05 \\
\hline 1976 & : & 6.32 & 6.82 & 6.69 & & & 7.04 & 7.72 & 7.86 & 7.53 & 7.45 & 5.67 & 5.62 \\
\hline 1977 & : & 6.15 & 6.91 & & 8.37 & 8.50 & & 10.15 & 83 & 93 & 4.48 & 86 & 07 \\
\hline 201 & $\vdots$ & 0.12 & 0.01 & & 0.37 & 0.50 & & 10.17 & .0s & . & 4.40 & & . \\
\hline 1978 & : & 6.32 & 6.45 & 6.98 & 6.86 & & 6.93 & 6.73 & 7.94 & 6.89 & 5.01 & 3.88 & 5.00 \\
\hline 1979 & : & 3.20 & 5.74 & 5.30 & 5.95 & 5.48 & 4.60 & 2.85 & 4.37 & & 3.15 & 3.08 & 4.18 \\
\hline 1980 & : & & 4.04 & 4.34 & 4.37 & 4.74 & 3.45 & 4.76 & 5.29 & 6.07 & 5.20 & 5.52 & 5.34 \\
\hline 1981 & $:$ & 5.96 & & 6.57 & 6.64 & 6.21 & 4.96 & 6.15 & 5.85 & 5.96 & 5.32 & 5.25 & 5.68 \\
\hline 1982 & : & 5.62 & 5.58 & 6.00 & 5.66 & 4.46 & 4.87 & 5.75 & 5.36 & 5.42 & 6.01 & 5.51 & 5.51 \\
\hline 1983 & $:$ & 5.96 & 6.42 & 6.17 & 6.60 & 6.12 & & 7.07 & 7.04 & 6.99 & 7.25 & 6.94 & 7.20 \\
\hline 1984 & : & 7.04 & 6.49 & 6.75 & 6.91 & 7.20 & 5.87 & 6.51 & 7.15 & 6.10 & 4.83 & 5.56 & 5.77 \\
\hline 1985 & & 5.63 & 6.59 & 6.53 & & & & & & & & & \\
\hline
\end{tabular}

REMARKS: Well discontinued.

a pumping, b recently pumped, e estimated, $h$ tape measurement, $j$ lowest water level recorded, $n$ nearby pumping well 


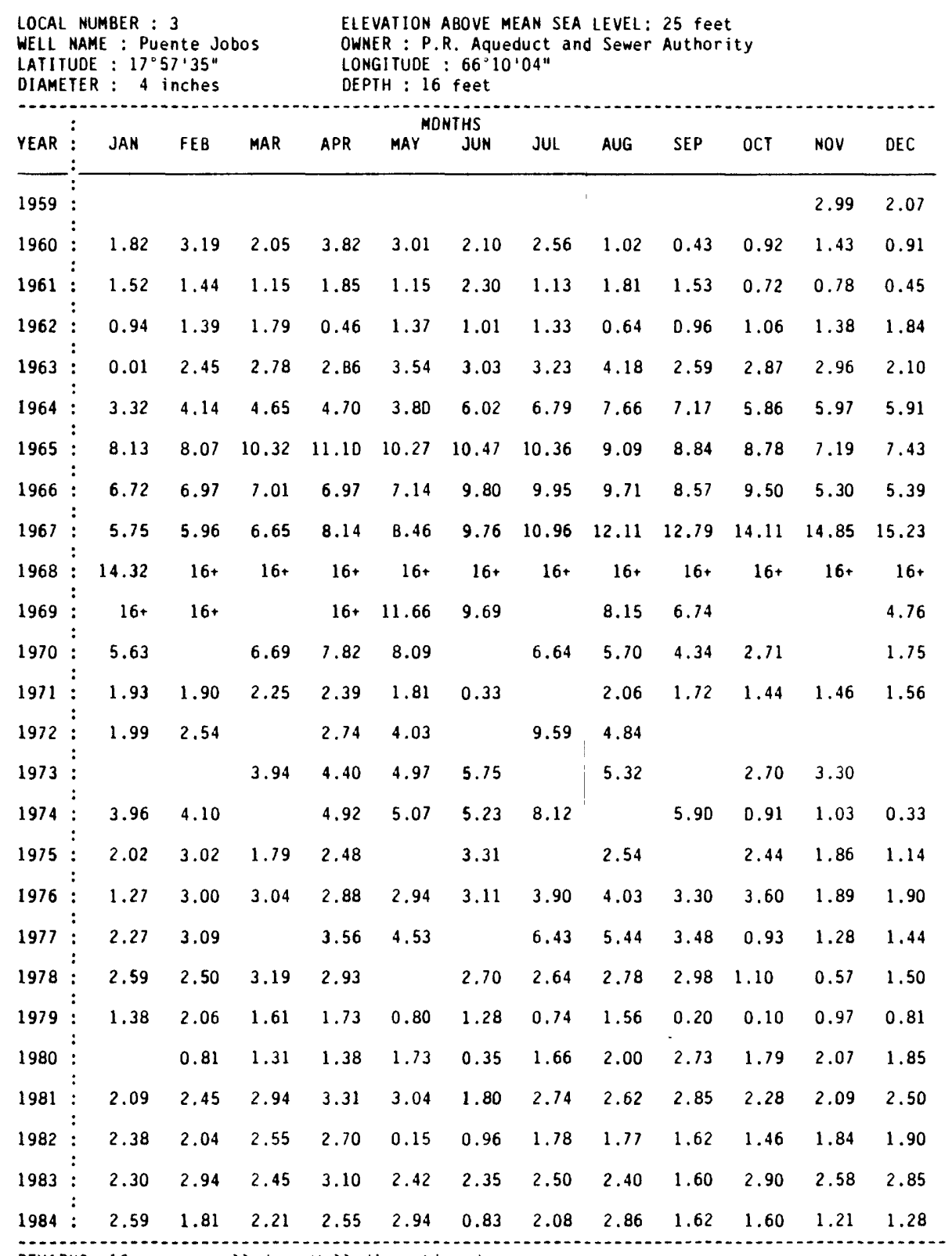

REMARKS: $16+$ aeans well dry. Well discont inued.

a pumping, b recent ly puaped, e estiated, $h$ tape measureaent, $j$ lowest water level recorded, n nearby puaping well 


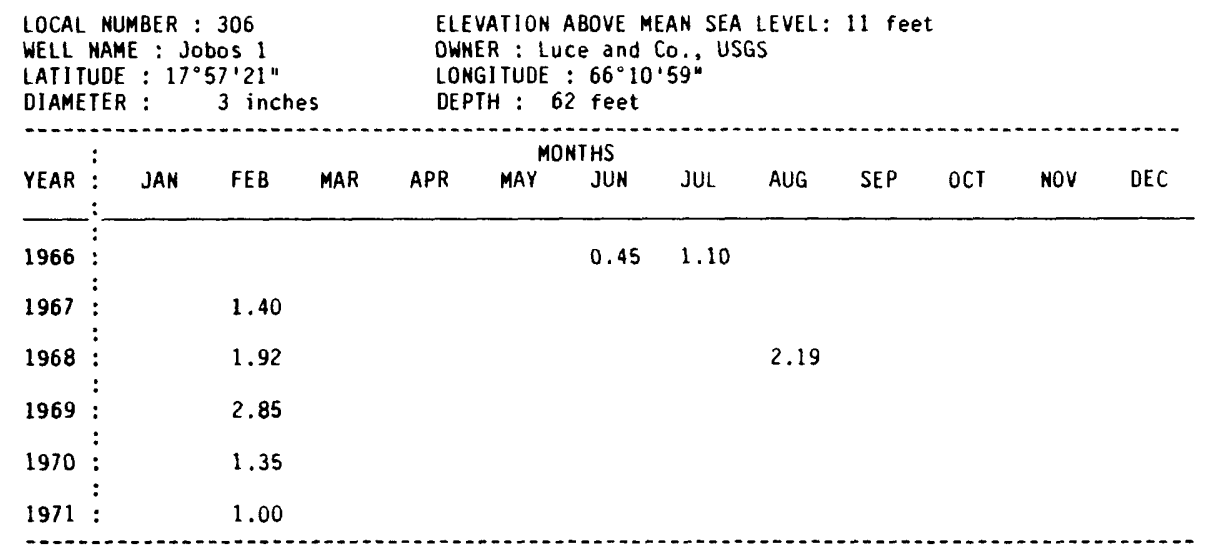

\begin{tabular}{|c|c|c|c|c|c|c|c|c|c|c|c|c|}
\hline \multicolumn{4}{|c|}{$\begin{array}{l}\text { LOCAL NUMBER : } 305 \\
\text { WELL NAME : JobOS } 2 \\
\text { LATI TUDE : } 17^{\circ} 57^{\prime} 23^{\prime \prime} \\
\text { DIAMETER : } 3 \text { inches }\end{array}$} & \multicolumn{7}{|c|}{$\begin{array}{l}\text { ELEVATION ABOVE MEAN SEA LEVEL: } 13 \text { feet } \\
\text { OWNER : LUCe and Co. USGS } \\
\text { LONGITUDE : } 66^{\circ} 10^{\prime} 08^{\prime \prime} \\
\text { DEPTH: } 99 \text { feet }\end{array}$} & & \multirow{3}{*}{ DEC } \\
\hline & : & & & & & & & & & & & \\
\hline YEAR & JAN & FEB & MAR & APR & MAY & JUN & JUL & AUG & SEP & $\mathrm{OCT}$ & NOV & \\
\hline 1966 & : & & & & & 6.50 & 5.10 & & & & & \\
\hline 1967 & : & 4.18 & & & & & & & & & & \\
\hline & : & & & & & & & & & & & \\
\hline 1968 & : & 7.67 & & & & & & 11.10 & & & & \\
\hline 1969 & : & 10.98 & & & & & & & & & & \\
\hline 1970 & : & 5.23 & & & & & & & & & & \\
\hline & : & & & & & & & & & & & \\
\hline 1971 & : & 4.18 & & & & & & & & & & \\
\hline 1972 & $\vdots$ & 3.42 & & & & & & & & & & \\
\hline
\end{tabular}

\begin{tabular}{|c|c|c|c|c|c|c|c|c|c|c|c|c|}
\hline \multirow{2}{*}{$\begin{array}{l}\text { LOCAL } \\
\text { WELL } \\
\text { LATIT } \\
\text { DIAME }\end{array}$} & \multicolumn{3}{|c|}{$\begin{array}{l}\text { NUMBER : } 307 \\
\text { NAME : Jobos } 7 \\
\text { UDE : } 17^{\circ} 57^{\prime} 49^{\prime \prime} \\
\text { TER : } 3 \text { inches }\end{array}$} & & $\begin{array}{l}\text { ATIOH } \\
R=1 \\
\text { I TUDE } \\
H: 1\end{array}$ & $\begin{array}{l}\text { ABOVE } \\
\text { raldo } \\
66^{\circ} 1 \\
\text { feet }\end{array}$ & $\begin{array}{l}\text { EAN SEA } \\
\text { en ito } \\
\text { '10" }\end{array}$ & $\begin{array}{l}\text { LEVEL: } \\
\text { SGS }\end{array}$ & $27 \mathrm{fe}$ & & & \\
\hline & : & & & & & NIHS & & & & & & \\
\hline YEAR & JAK & FEB & MAR & APR & MAY & JUN & JUL & AUG & SEP & $O C T$ & NOV & DEC \\
\hline 1966 & $\vdots$ & & & & & 17.30 & 22.90 & & & & & \\
\hline 1967 & : & 18.40 & & & & & & & & & & \\
\hline 1968 & : & 15.90 & & & & & & 19.17 & & & & \\
\hline & : & & & & & & & & & & & \\
\hline 1969 & : & 18.21 & & & & & & & & & & \\
\hline 1970 & $\vdots$ & 9.95 & & & & & & & & & & \\
\hline & : & & & & & & & & & & & \\
\hline 1971 & : & -0.05 & & & & & & & & & & \\
\hline & : & & & & & & & & & & & \\
\hline 1972 & : & 1.10 & & & & & & & & & & \\
\hline
\end{tabular}

a pumping, b recently pumped, e estimated, $h$ tape measurement, $j$ lowest water level recorded, n nearby pumping well 


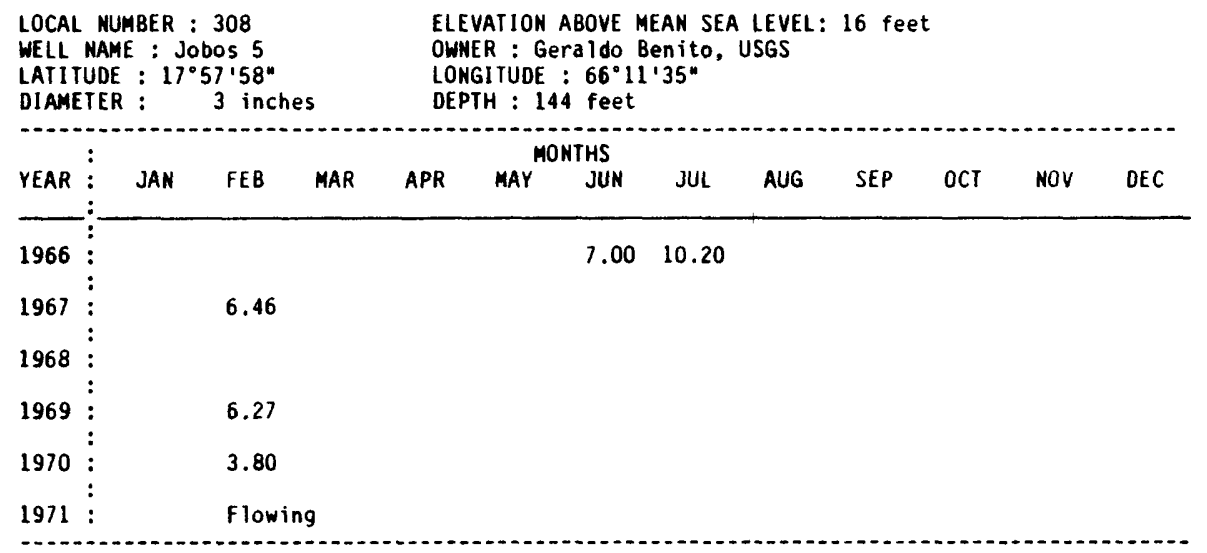

Reark: Flowing when visited February 25, 1971.

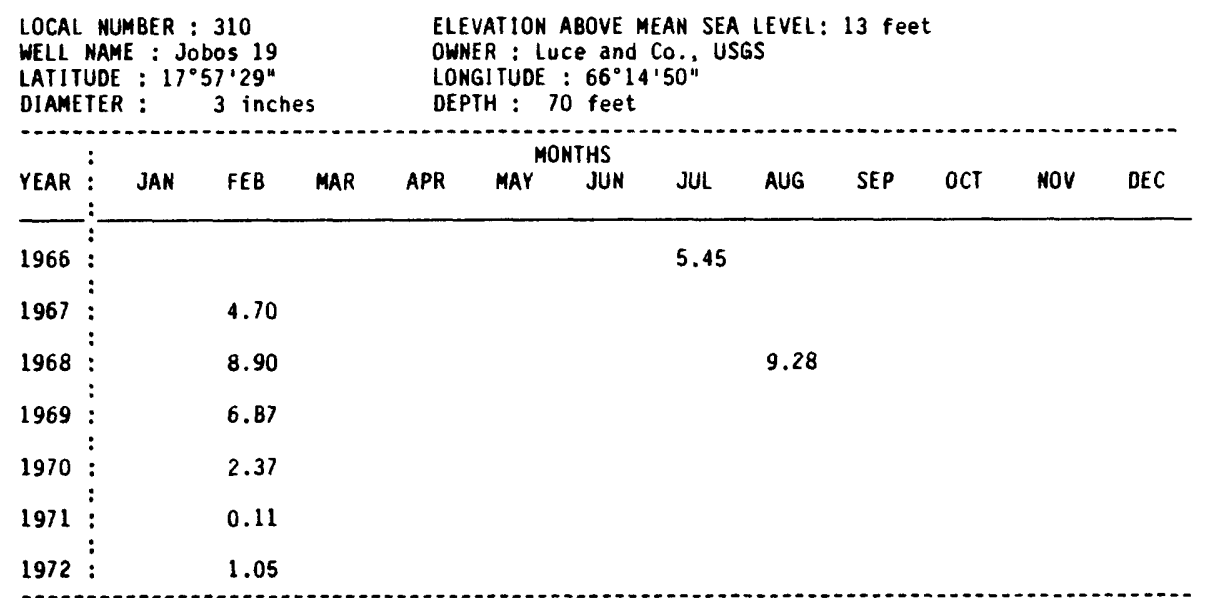

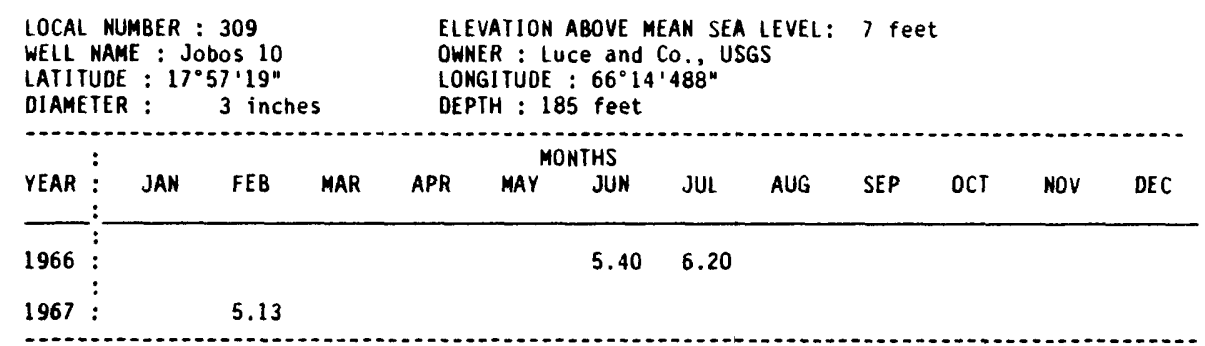

a pumping, b recently pumped, e estimated, $h$ tape measurement, $j$ lowest water level recorded, n nearby puaping well 


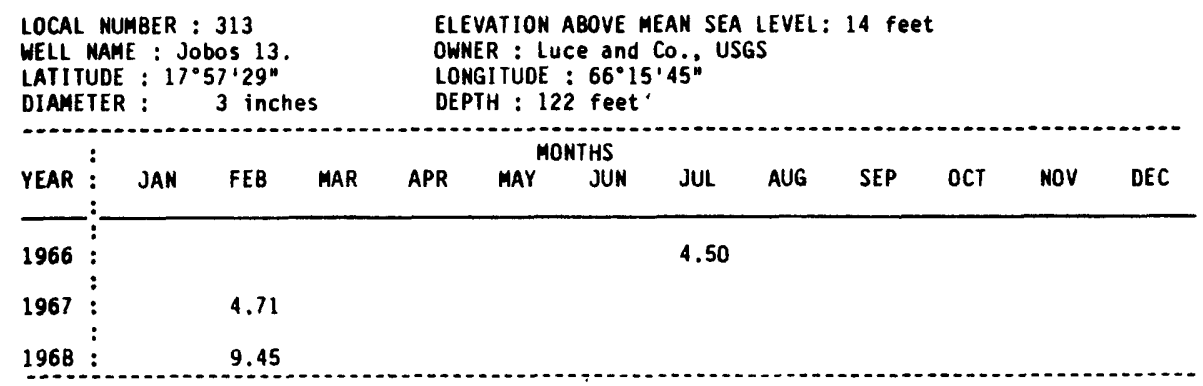

REMARKS: Well found destroyed, February 1969.

\begin{tabular}{|c|c|c|c|c|c|c|c|c|c|c|c|c|}
\hline \multicolumn{4}{|c|}{$\begin{array}{l}\text { LOCAL NUMBER : } 312 \\
\text { WELL NAME : Jobos } 12 \\
\text { LATITUDE : } 17^{\circ} 57^{\prime} 51^{\prime \prime} \\
\text { DIAMETER : } 3 \text { inches }\end{array}$} & \multicolumn{7}{|c|}{$\begin{array}{l}\text { ELEVATION ABOVE MEAN SEA LEVEL: } 22 \text { feet } \\
\text { OWMER : Luce and Co., USGS } \\
\text { LONGITUDE : } 66^{\circ} 15^{\prime} 23^{\circ} \\
\text { DEPTH : } 108 \text { feet }\end{array}$} & \multirow[b]{2}{*}{ NOV } & \multirow[b]{2}{*}{ DEC } \\
\hline YEAR & JAN & FEB & MAR & APR & MAY' & $\begin{array}{l}\text { NTHS } \\
\text { JUN }\end{array}$ & JUL & AUG & SEP & OCT & & \\
\hline 1966 & & & & & & 19.50 & 19.80 & & & & & \\
\hline 1967 & & 14.10 & & & & & & & & & & \\
\hline 1968 & & 20.90 & & & & & & 23.34 & & & & \\
\hline 1969 & & 7.99 & & & & & & & & & & \\
\hline 1970 & & 3.55 & & & & & & & & & & \\
\hline 1971 & & 1.77 & & & & & & & & & & \\
\hline 1972 & & 3.65 & & & & & & & & & & \\
\hline
\end{tabular}

\begin{tabular}{|c|c|c|c|c|c|c|c|c|c|c|c|c|}
\hline $\begin{array}{l}\text { LOCAL } \\
\text { WELL } \\
\text { LATIT } \\
\text { DIAME }\end{array}$ & $\begin{array}{l}\text { MUMBER } \\
\text { NAME : } \\
\text { IUDE : } 1 \\
\text { ETER : }\end{array}$ & $\begin{array}{l}311 \\
\text { os } 11 \\
7 \cdot 22 " \\
3 \text { inc }\end{array}$ & & $\begin{array}{l}\text { EL } \\
\text { OW } \\
\text { LO } \\
\text { DE }\end{array}$ & $\begin{array}{l}\text { AT ION } \\
\text { R : L } \\
\text { ITUDE } \\
H: 1\end{array}$ & $\begin{array}{l}\text { ABOVE } \\
\text { ce and } \\
66^{\circ} 1 \\
8 \text { feet }\end{array}$ & $\begin{array}{l}A \text { AN SE } \\
0 ., \mathrm{U} \\
20^{4}\end{array}$ & EVEL : & $8 \mathrm{ft}$ & & & \\
\hline YEAR & : & FEB & MAR & APR & MAY & $\begin{array}{l}\text { NTHS } \\
\text { JUN }\end{array}$ & JUL & AUG & SEP & OCT & NOV & DEC \\
\hline 1966 & $\vdots$ & & & & & 4.90 & 5.15 & & & & & \\
\hline 1967 & : & 3.59 & & & & & & & & & & \\
\hline
\end{tabular}

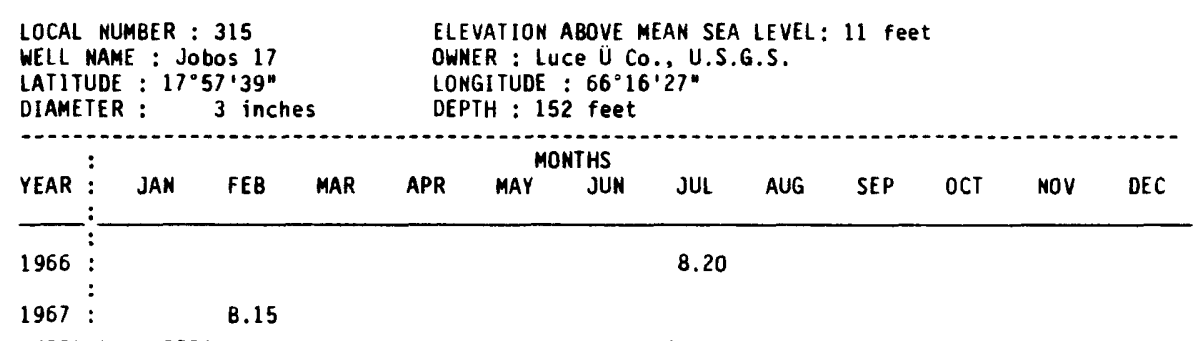

a pumping, b recently pumped, e estimated, $h$ tape measurement, $j$ lowest water level recorded, n nearby pumping well 


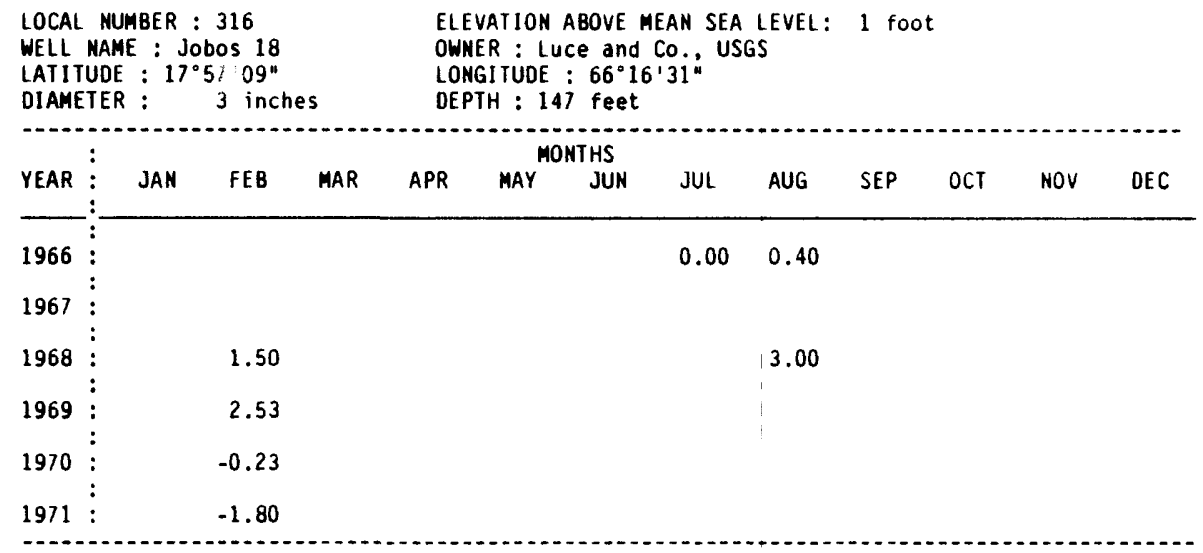

REMARKS: Well flowing during $02 / 17 / 1971$. Maximun head above LSO is 1.8 feet.

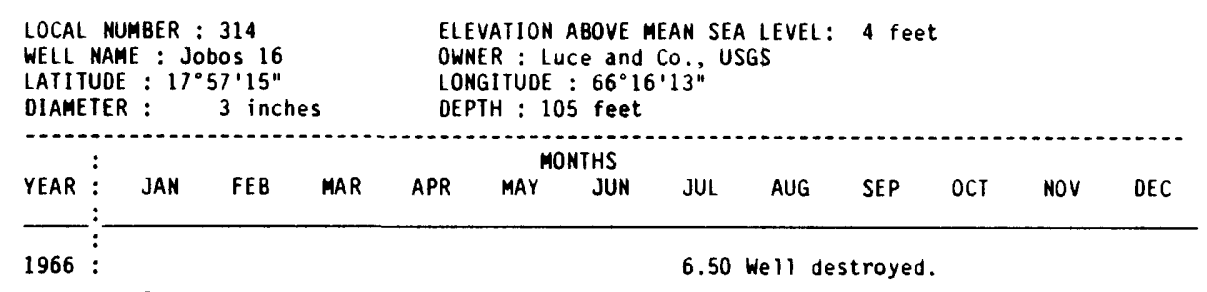

Remarks: Well destroyed, August, 1966.

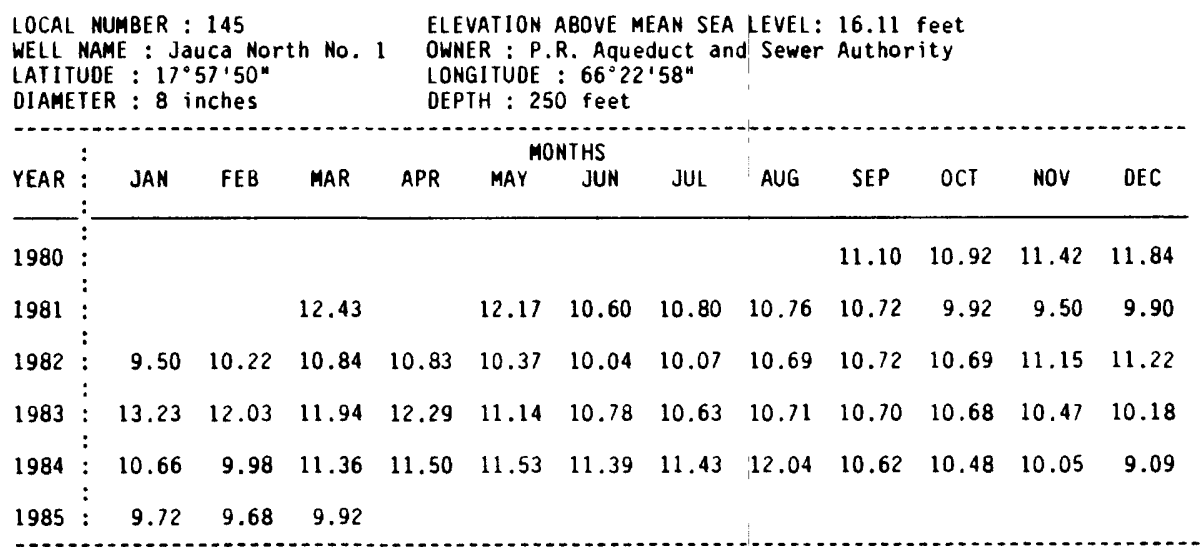

REMARKS: Well discontinued.

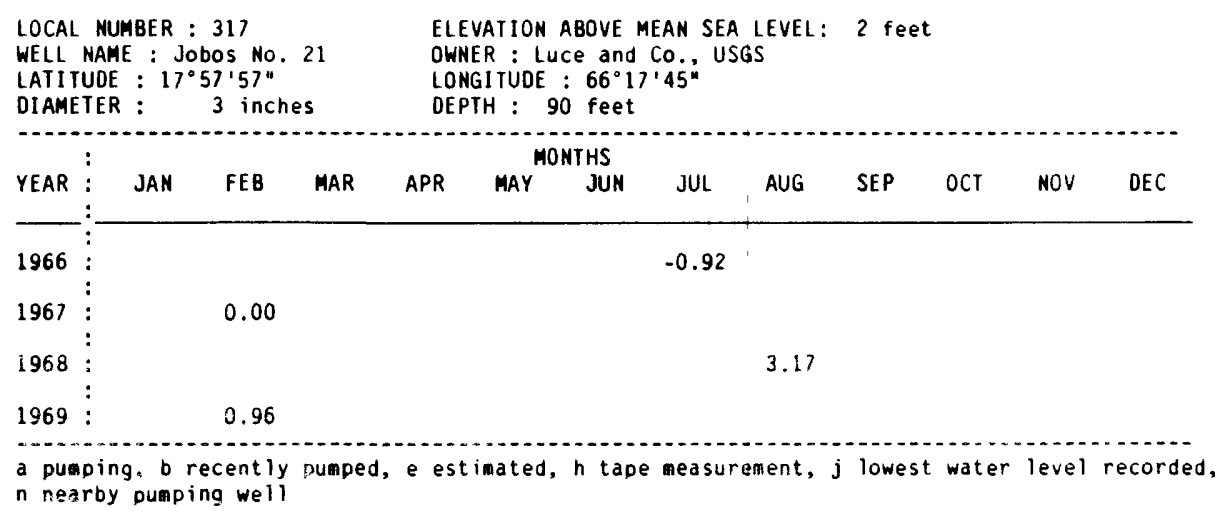




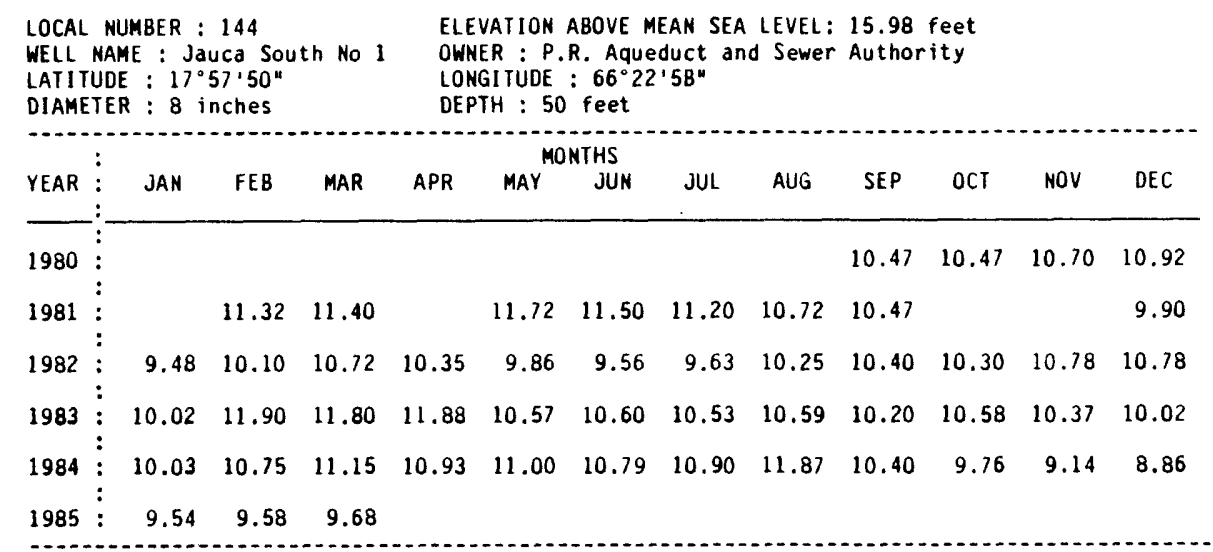

REMARKS: Well discont inued.

\begin{tabular}{|c|c|c|c|c|c|c|c|c|c|c|c|c|}
\hline $\begin{array}{l}\text { LOCAL } \\
\text { WELL } \\
\text { LATIT } \\
\text { DIAME }\end{array}$ & $\begin{array}{l}\text { NUMBER : } \\
\text { NAME : Ha } \\
\text { UDE : } 17^{\circ} \\
\text { TER : } 8\end{array}$ & $\begin{array}{l}146 \\
\text { c. Alom } \\
57 \cdot 34^{\prime \prime} \\
\text { nches }\end{array}$ & Wes & $\begin{array}{l}\text { ELE } \\
\text { \#3 OWN } \\
\text { LON } \\
\text { DEP }\end{array}$ & $\begin{array}{l}\text { ATION } \\
R: P \text {. P. } \\
\text { ITUDE } \\
H: 70\end{array}$ & $\begin{array}{l}\text { BOVE M } \\
\text { R. Aque } \\
66^{\circ} 23 \\
\text { feet }\end{array}$ & $\begin{array}{l}\text { AN S } \\
\text { Jct } \\
33^{\prime \prime}\end{array}$ & LEVEL & Autho & feet & & \\
\hline & : & & & & & THS & & & & & & \\
\hline YEAR & JAN & FEB & MAR & APR & MAY & JUN & JUL & AUG & SEP & $\mathrm{OCT}$ & NOV & DEC \\
\hline 1981 & : & & & & & 8.98 & 11.53 & 12.49 & 11.35 & & & 10.17 \\
\hline 1982 & 9.09 & 10.65 & 11.59 & 10.69 & 9.93 & 10.27 & 10.06 & 10.84 & 10.37 & 9.49 & 10.18 & 9.92 \\
\hline 1983 & $: \quad 10.30$ & 9.78 & 10.64 & 12.17 & 11.06 & 8.96 & 9.55 & 10.38 & 11.00 & 11.13 & 9.23 & 9.07 \\
\hline 1984 & 9.27 & 10.00 & 10.29 & 10.50 & 10.69 & 9.74 & 10.30 & 11.56 & 9.04 & 7.43 & 8.78 & 8.95 \\
\hline 1985 & 9.22 & 9.29 & 8.37 & & & & & & & & & \\
\hline
\end{tabular}

$1985: 9.22 \quad 9.29 \quad 8.37$

REMARKS: Well discont inued.

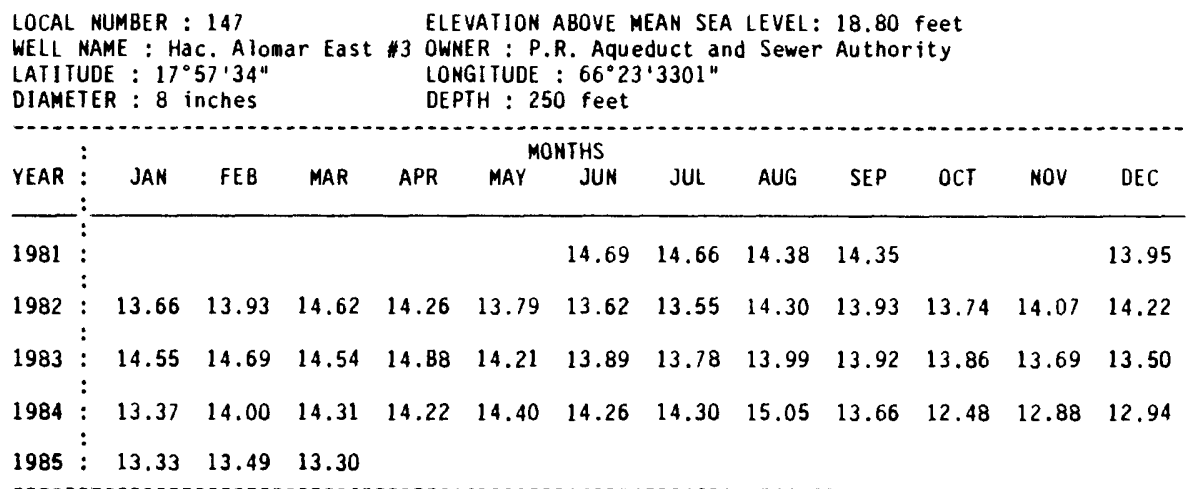

REMARKS: Well discont inued.

a pumping, b recently pumped, e estimated, $h$ tape measurement, $j$ lowest water level recorded, $n$ nearby pumping well 


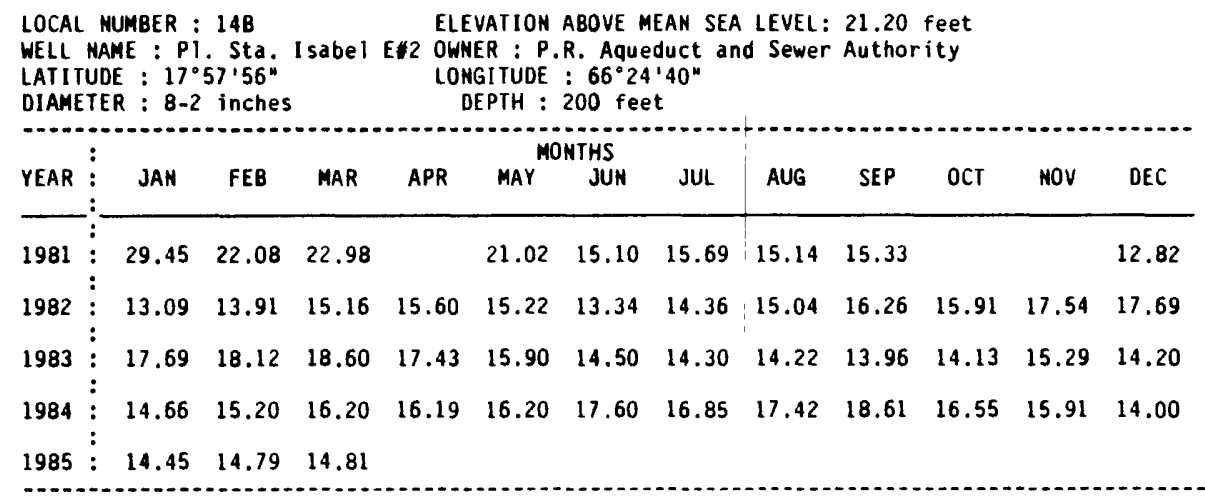

REMARKS: Well discontinued, April, 1985.

LOCAL NUMBER : 149

ELEVATION ABOVE MEAN SEA LEVEL: 21.20 feet

WELL MAME : PI. Sta. I sabel W\#2 OWNER : P.R. Aqueduct and Sewer Authority

LATITUDE : $17^{\circ} 57^{\prime} 56^{\prime \prime}$ LONGITUDE : $66^{\circ} 24^{\prime} 4001^{\prime \prime}$

DIAMETER : $8-2$ inches DEPTH : 250 feet

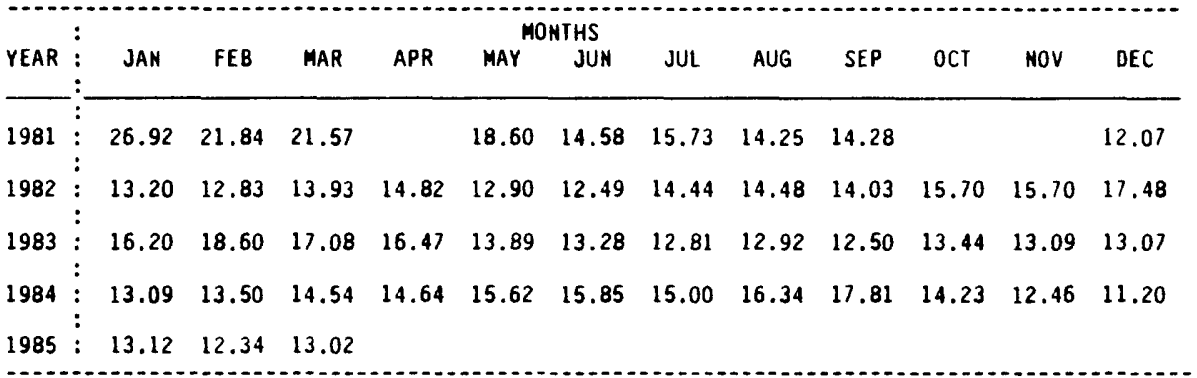

REMARKS: Well discontinued, April, 1985.

a pumping, b recently pumped, e estimated, $h$ tape measurement, $j$ lowest water level recorded, n nearby pumping well 


\begin{tabular}{|c|c|c|c|c|c|c|c|c|c|c|c|c|c|}
\hline $\begin{array}{l}\text { LOCAL } \\
\text { WELL } \\
\text { LATIT } \\
\text { OIAME }\end{array}$ & & $\begin{array}{l}\text { UMBER : } \\
\text { ME : Ju } \\
E: 17^{\circ} \\
R: 16\end{array}$ & $\begin{array}{l}6 \\
\text { na } 5 \\
58^{\prime} 58^{\prime \prime} \\
\text { inches }\end{array}$ & & $\begin{array}{l}\text { ELE } \\
\text { OWN } \\
\text { LON } \\
\text { DEP }\end{array}$ & $\begin{array}{l}\text { VATION } \\
\text { ER : Dr } \\
\text { GITUOE } \\
\text { TH : } 17\end{array}$ & $\begin{array}{l}\text { ABOVE } \\
\text { Brung } \\
\vdots 66^{\circ} 10 \\
3 \text { feet }\end{array}$ & $\begin{array}{l}\text { AN SEA } \\
\text { 2" }\end{array}$ & LEVEL & 127 & & & \\
\hline 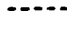 & & & & & & $\mathrm{Mn}$ & THS & & & & & & \\
\hline YEAR & : & JAN & FEB & MAR & APR & MAY & JUN & JUL & AUG & SEP & $\mathrm{OCT}$ & NOV & DEC \\
\hline 1960 & $:$ & & & & & & & & & & & 29.76 & \\
\hline 1961 & : & & & & & & & 45.99 & $46.0 \mathrm{~B}$ & 43.07 & 42.60 & $34 . \mathrm{Bl}$ & 35.46 \\
\hline 1962 & $\vdots$ & 39.42 & 42.25 & 45.05 & $45 . B 8$ & 46.67 & 48.09 & 49.18 & 49.15 & 47.16 & 46.61 & $43.6 \mathrm{~B}$ & 45.44 \\
\hline 1963 & : & 46.09 & 50.73 & & 52.41 & 51.51 & 50.53 & 50.43 & 51.80 & 51.36 & 50.51 & 52.31 & 52.73 \\
\hline 1964 & : & 54.00 & 54.79 & 56.21 & 56.36 & 55.67 & 57.40 & 57.49 & 57.72 & 58.13 & 57.47 & 54.30 & 55.58 \\
\hline 1965 & : & & 59.19 & 61.13 & 60.77 & 61.46 & 60.13 & 56.75 & 56.85 & 56.10 & 53.16 & 53.68 & 55.18 \\
\hline 1966 & : & $55 . \mathrm{B3}$ & 57.37 & 56.86 & & 57.65 & 56.55 & 54.75 & 54.35 & 52.10 & 51.95 & 50.55 & 49.45 \\
\hline 1967 & : & 50.85 & 52.70 & 55.10 & 58.90 & 58.95 & 61.40 & 62.30 & 62.65 & 62.90 & 63.75 & 63.80 & 63.75 \\
\hline 1968 & : & 64.45 & 63.90 & 64.55 & 65.05 & 65.90 & 65.50 & 64.15 & 62.85 & 62.90 & 60.88 & 60.98 & 61.93 \\
\hline 1969 & : & 62.40 & 61.65 & 62.35 & 62.22 & 63.05 & 62.95 & 63.15 & 59.25 & 53.30 & & & 49.30 \\
\hline 1970 & : & 49.10 & 49.90 & 51.35 & 52.60 & 54.60 & 56.15 & 57.20 & 58.50 & 58.40 & 53.60 & 25.65 & \\
\hline 1971 & : & & 35.25 & 38.90 & 39.55 & 44.15 & 44.60 & 43.85 & 44.35 & 45.61 & 47.28 & 48.69 & 50.22 \\
\hline 1972 & : & 52.65 & 54.26 & 54.97 & 56.94 & 58.72 & 59.39 & 59.19 & 55.98 & 50.65 & 52.50 & 49.60 & \\
\hline 1973 & : & 47.78 & 48.52 & & & & & 56.25 & 57.58 & 56.52 & 54.29 & 54.03 & \\
\hline 1974 & : & 49.34 & 52.05 & 54.57 & 55.15 & 56.90 & 59.57 & 60.46 & 60.90 & 57.89 & 54.90 & 49.43 & 37.65 \\
\hline 1975 & $:$ & 35.95 & 36.68 & 42.05 & 44.76 & 46.73 & & & 51.18 & 51.52 & 50.37 & 49.92 & 49.43 \\
\hline 1976 & : & 47.91 & 48.32 & 49.64 & 50.65 & 51.33 & 52.06 & 52.25 & 52.68 & 52.79 & 51.40 & $50.60^{\mathrm{e}}$ & 51.18 \\
\hline 1977 & : & 52.02 & 52.99 & 55.74 & 56.96 & 52.69 & 53.62 & 54.78 & 56.05 & 57.60 & 55.84 & 52.36 & 47.11 \\
\hline 1978 & : & 45.55 & 46.17 & 49.77 & 50.20 & 52.60 & 48.58 & 49.55 & 50.89 & 51.33 & 53.44 & 41.07 & 40.79 \\
\hline & ? & & & & & & & & & & & & \\
\hline 1979 & : & 41.80 & ${ }_{\mathrm{e}}^{40.66}$ & 42.45 & 43.95 & 46.52 & 48.72 & 46.17 & 45.56 & 39.63 & 27.22 & 27.15 & .70 \\
\hline 1980 & : & 33.70 & 38.30 & 42.14 & 44.34 & 47.10 & 48.81 & 47.25 & 48.07 & 48.53 & 49.03 & 46.88 & 51.26 \\
\hline 1981 & : & 50.57 & 50.29 & 52.74 & 54.74 & 54.27 & 52.66 & 52.05 & 54.96 & 55.76 & 54.97 & 55.44 & 53.50 \\
\hline 1982 & : & 52.86 & 53.18 & 53.53 & 50.68 & 46.77 & 46.41 & 47.89 & 49.76 & 51.14 & 52.62 & 53.91 & 54.57 \\
\hline 1983 & : & 55.18 & 55.45 & 54.56 & 54.20 & 56.41 & 58.11 & 58.36 & 57.19 & 55.53 & 55.25 & 48.26 & 45.19 \\
\hline 1984 & : & 46.35 & 50.01 & 52.49 & 52.28 & 50.71 & 52.25 & 54.30 & 54.55 & 55.08 & 54.90 & 53.22 & 43.99 \\
\hline 1985 & : & 43.77 & 46.42 & 48.67 & 50.19 & 50.83 & 48.55 & 48.79 & 50.52 & 52.02 & & & \\
\hline
\end{tabular}

REMARKS: Depth 173 feet reported, 110 feet asured.

a puaping, b recently puaped, e estiated, $h$ tape measurement, $j$ lowest water level recorded, n nearby pusping well 
LOCAL MUMBER : 318

WELL MAME: Jobos No. 6

ELEVATION ABOVE MEAN SEA LEVEL: 11 feet

LATITUDE : $17^{\circ} 5 \mathrm{~B}^{\prime} 11^{\prime}$

OWNER : LUCE and CO., USGS

DIAMETER :

3 inches

LONGITUDE : $66^{\circ} 12^{\prime} 08^{\prime \prime}$

DEPTH : 62 feet

YEAR $:$ JAN FEB MAR APR MAY JUN JUL AUG SEP OCT NOV DEC

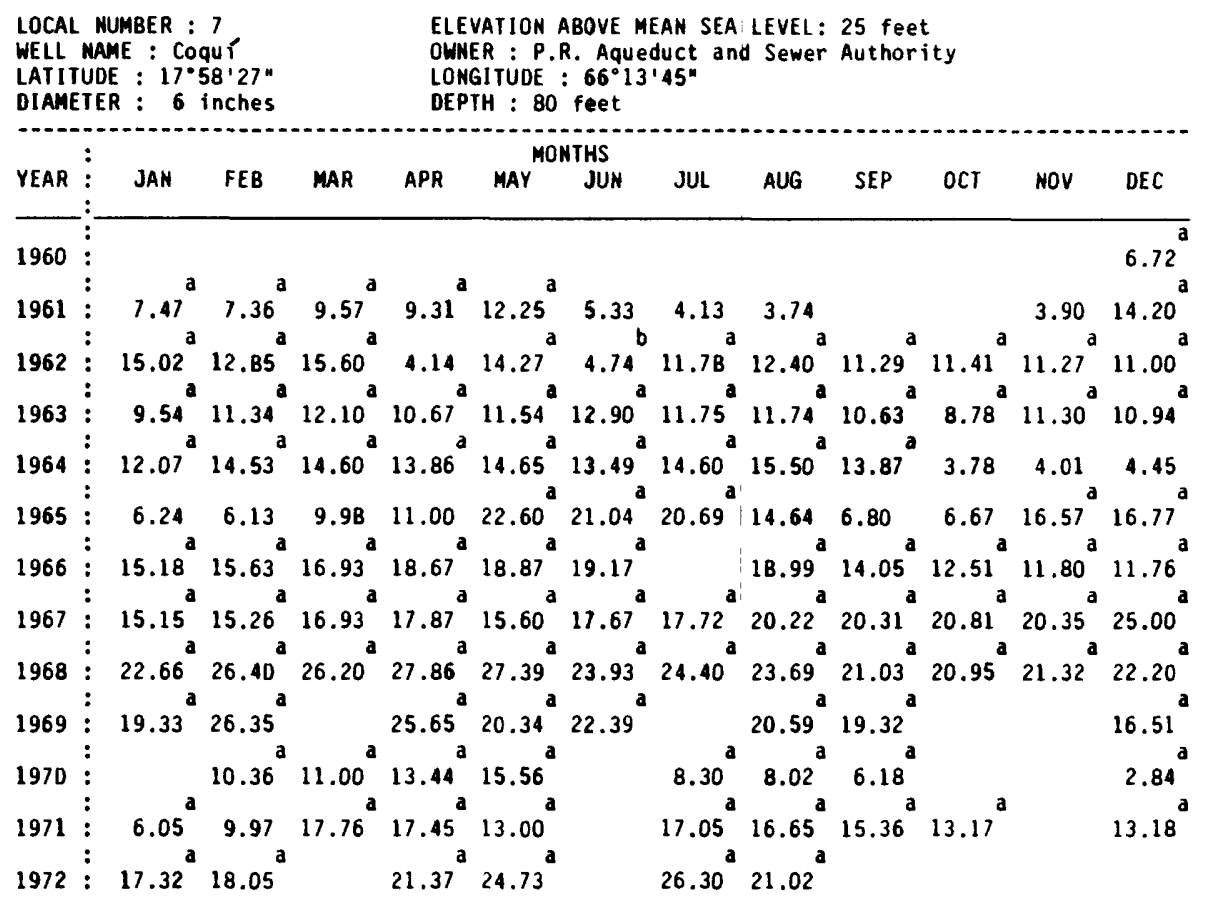

REMARKS: Hell discont inued Septeaber 1972

a pumping, b recently pumped, e estimated, $h$ tape measurement, $j$ lowest water level recorded, n nearby pumping well 


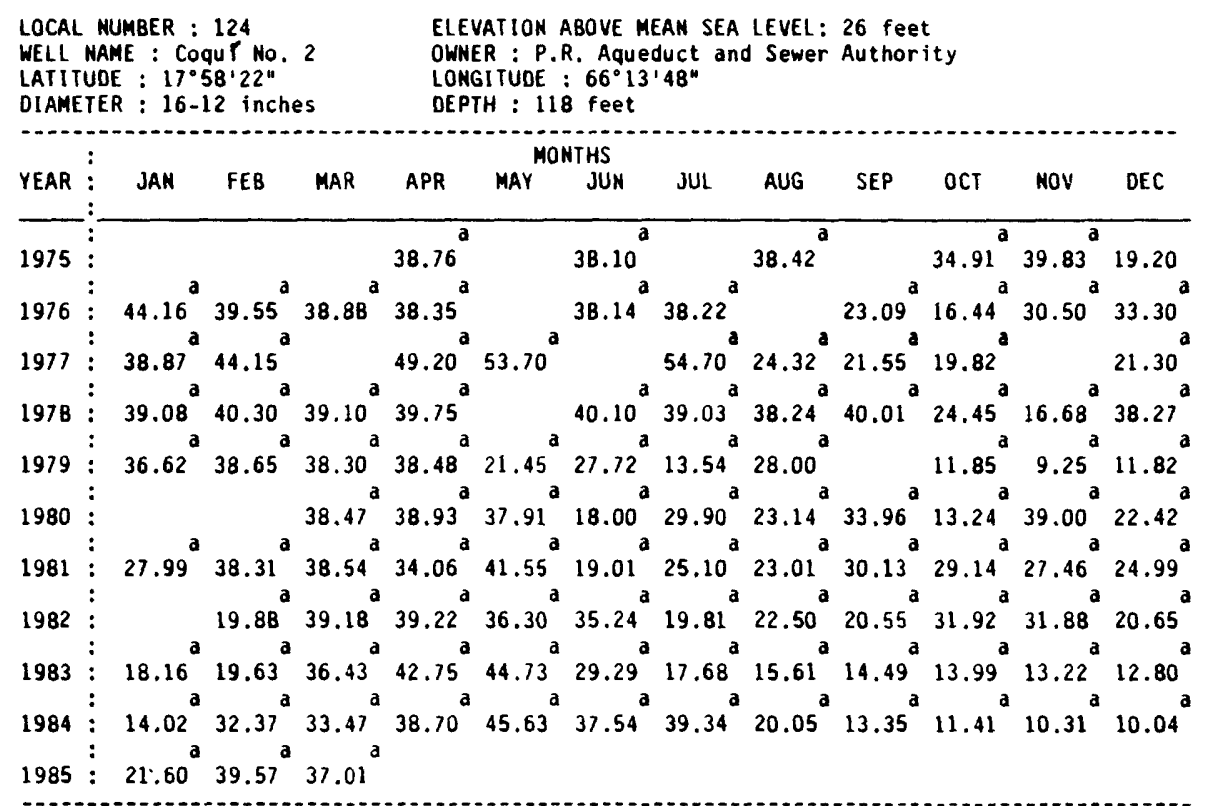

REMARKS: Well discontinued, April, 1985.

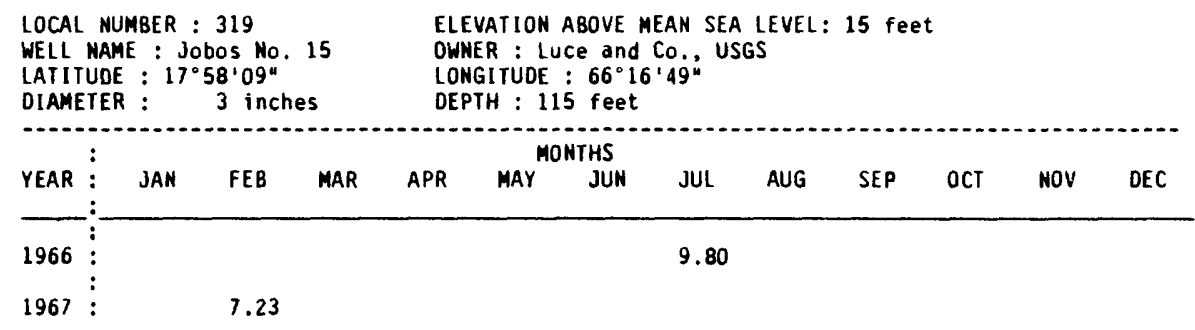

a pumping, b recently pumped, e estimated, h tape measurement, j lowest water level recorded, n nearby puaping well 


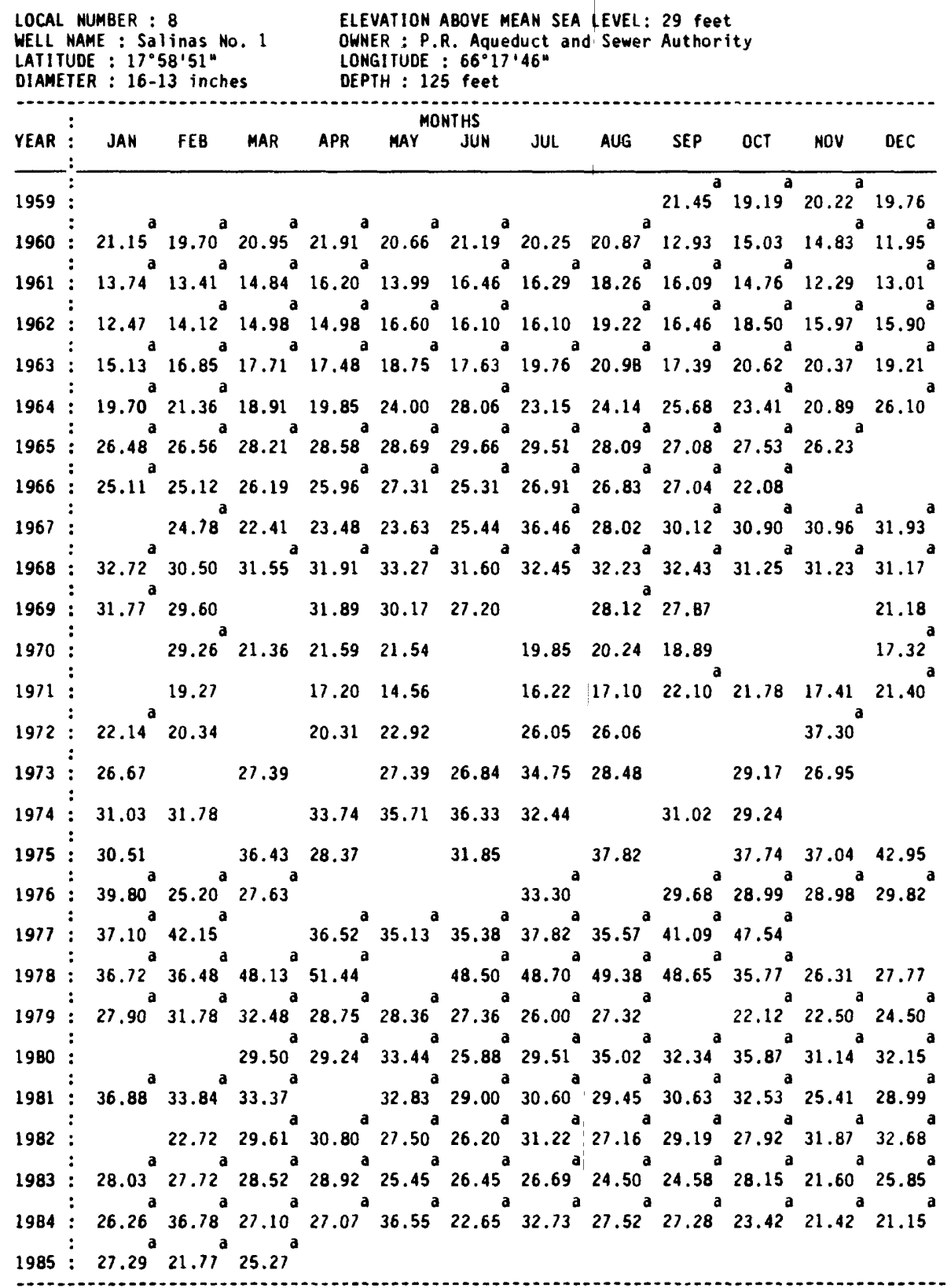

REMARKS: Well discont inued, April 1985.

a pumping, b recently pumped, e estimated, $h$ tape measurement, $j$ lowest water level recorded,

n nearby pumping well 


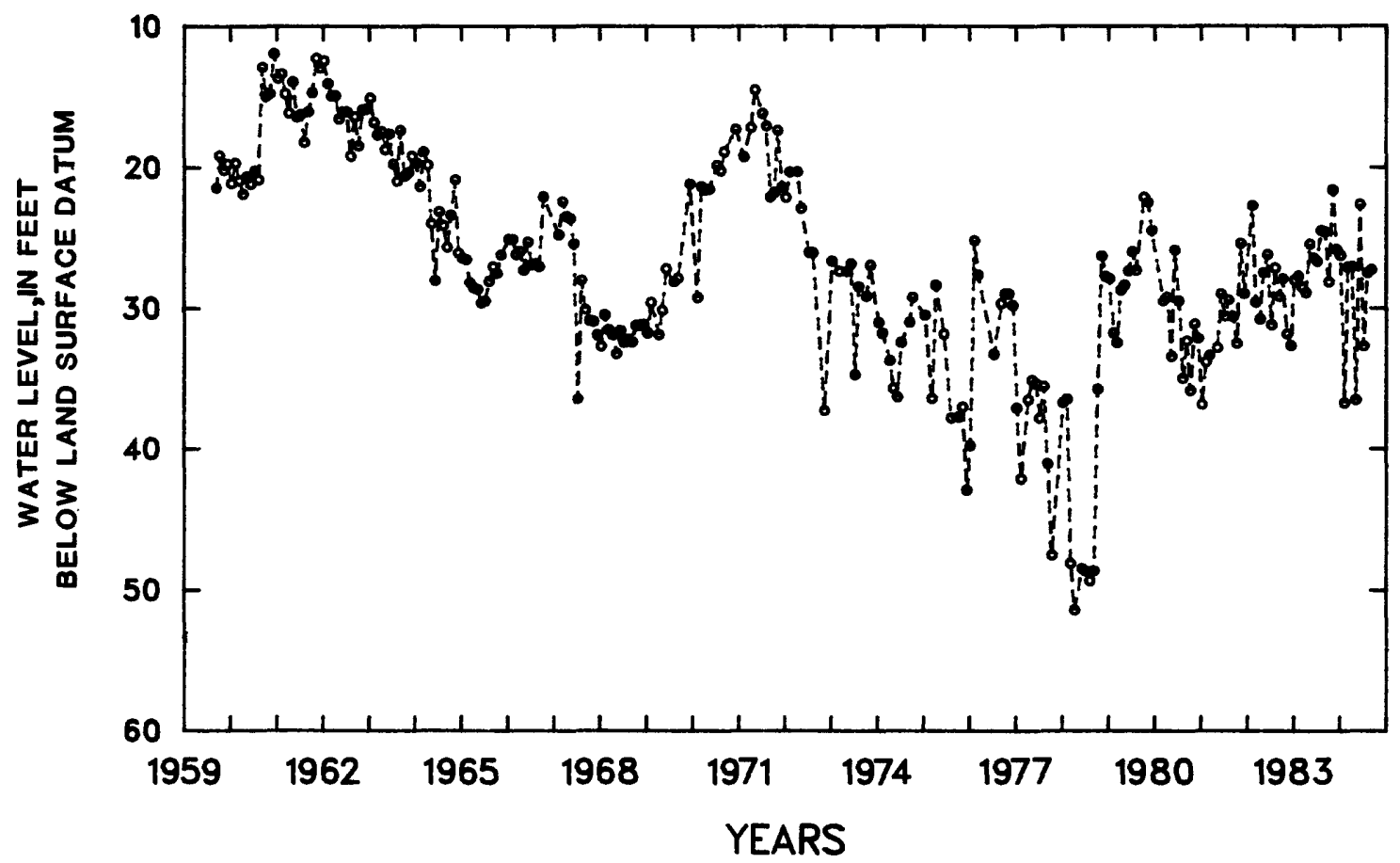

Figure 17.--Ground-water levels at Salinas, local number 8. 


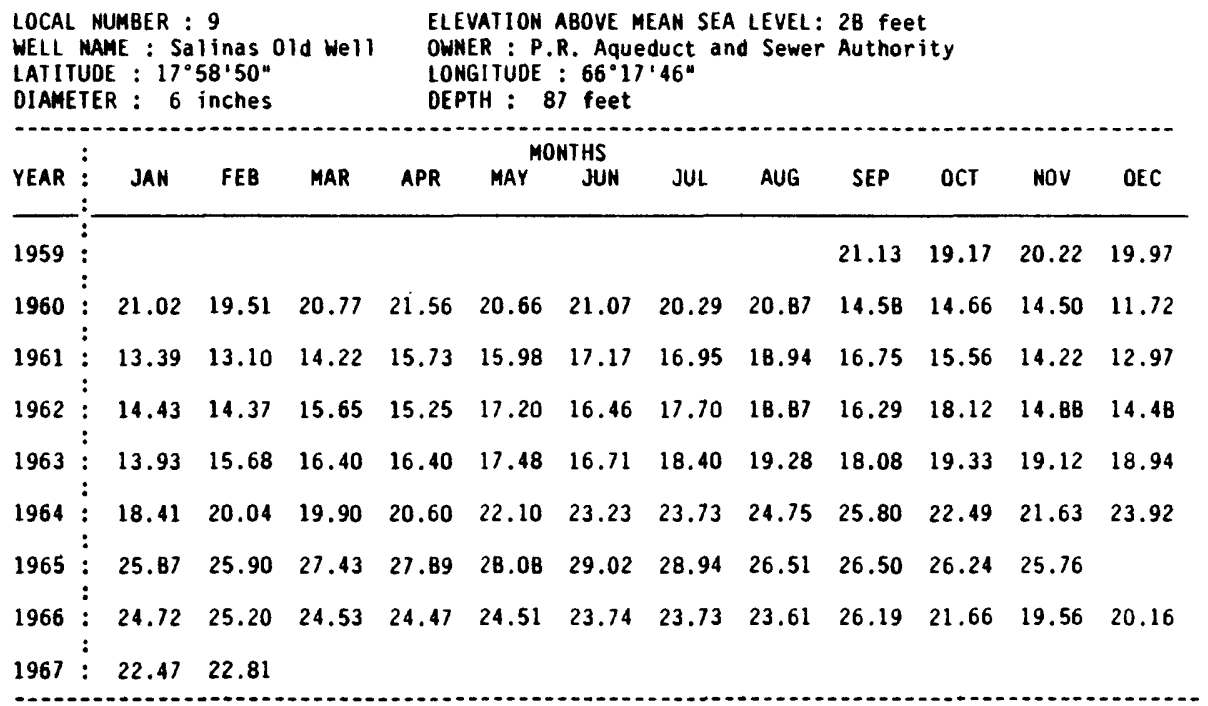

Reark: Affected by nearby pumping. Well discontinued, March 1967.

a puaping, b recently puaped, e estimated, $h$ tape measurement, $j$ lowest water level recorded, n nearby pumping well 


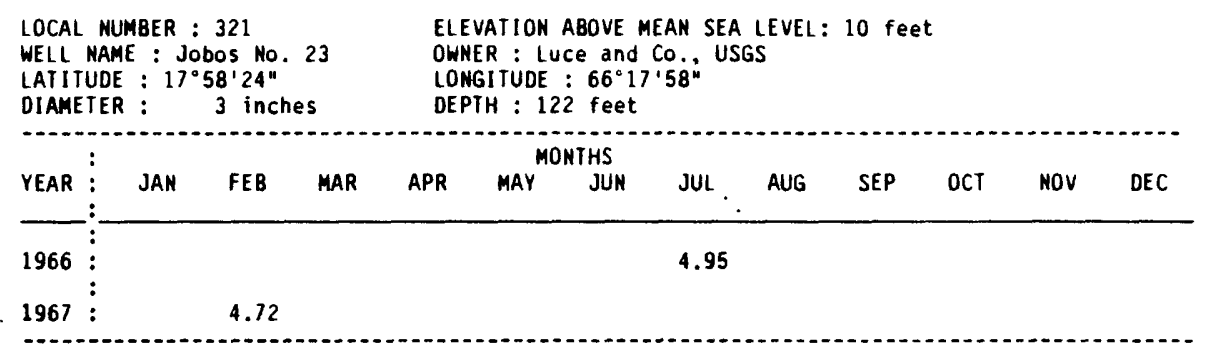

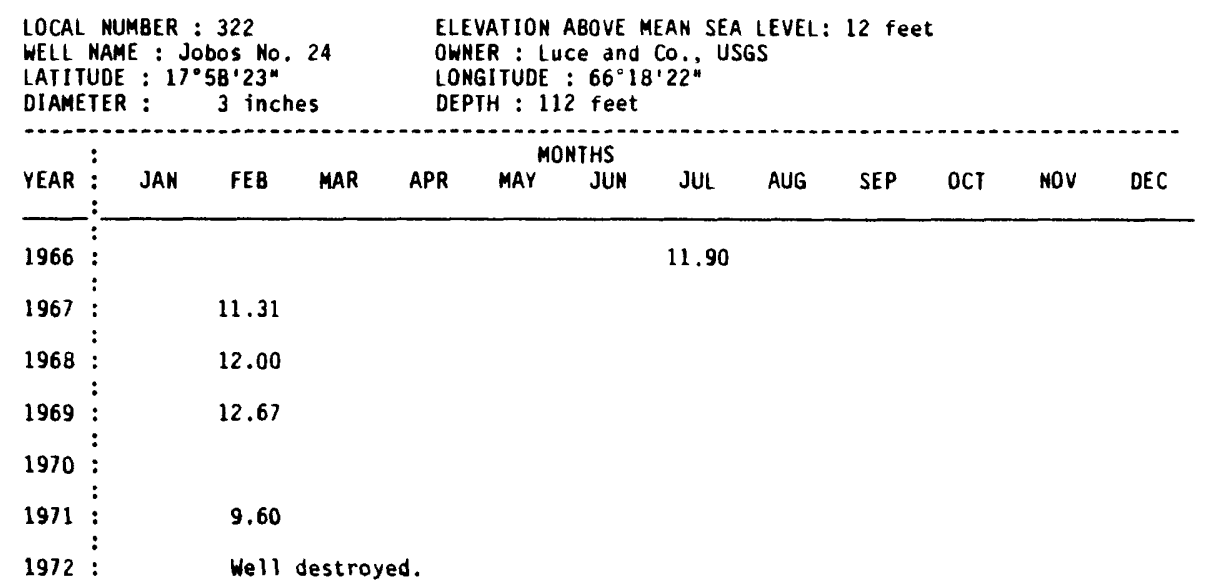

remark: Well destroyed $02 / 1972$

Renark: Well destroyed $02 / 1972$

a pumping, b recently pumped, e estimated, $h$ tape measurement, $j$ lowest water level recorded, n nearby punping well 
LOCAL NUMBER : 323

WELL NAME : Jobos No. 20

LATITUDE : $17^{\circ} 58^{\circ} 06^{\prime \prime}$

DIAMETER : 3 inches

ELEVATION ABOVE MEAN SEA LEVEL: 7 feet

OWNER : LUCE and CO. USGS

LONGITUDE : $66^{\circ} 17^{\prime} 23^{\circ}$

DEPTH : 120 feet

\begin{tabular}{llllllllll} 
YEAR & JAN FEB MAR APR MAY JUN JUL AUG SEP OCT NOV DEC \\
\hline $1966:$ & & & 2.30 & \\
$1967:$ & 0.93 & & &
\end{tabular}

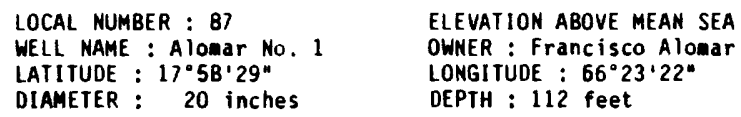




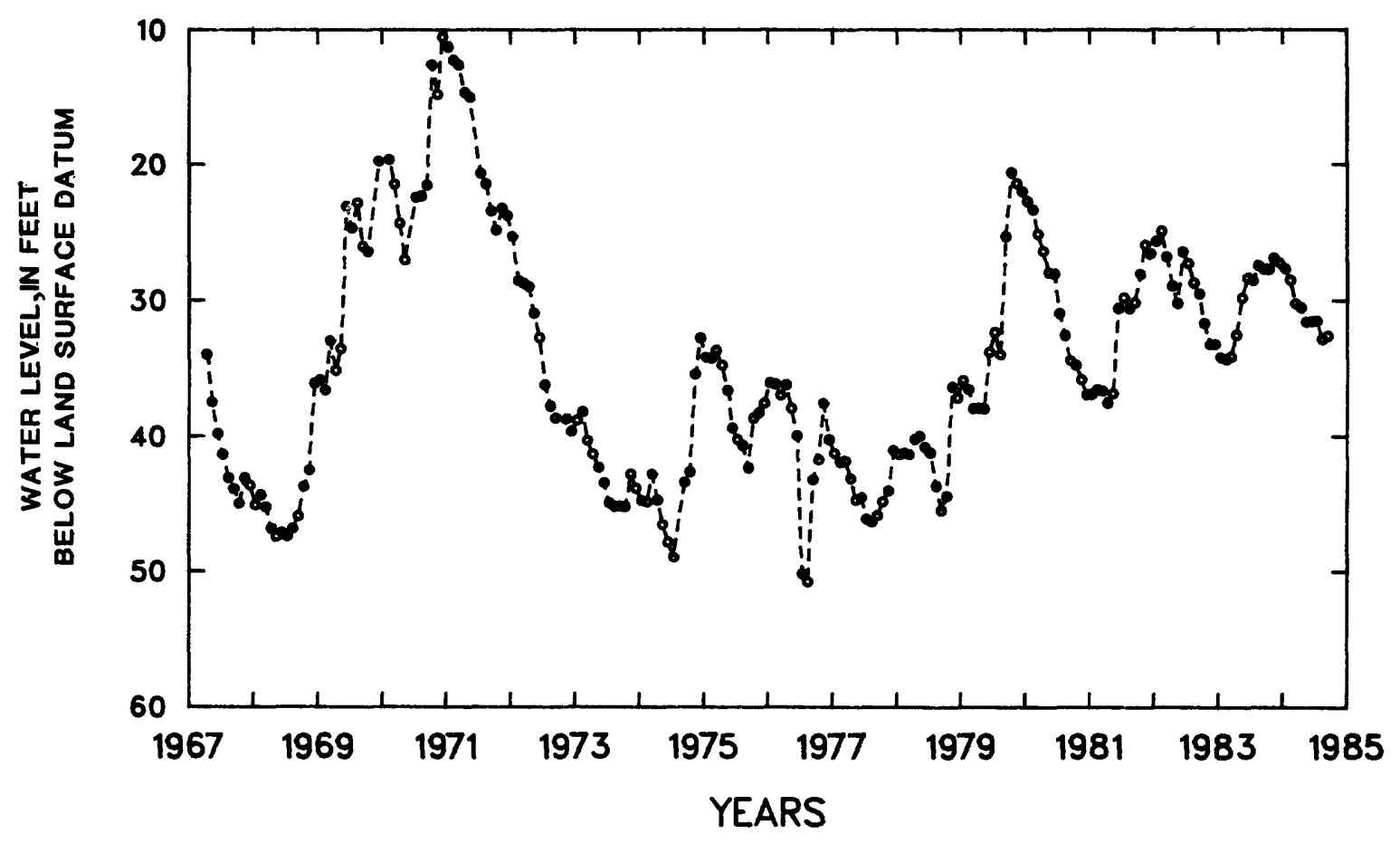

Figure 18.--Ground-water levels at Alomar Number 1. local number 87. 


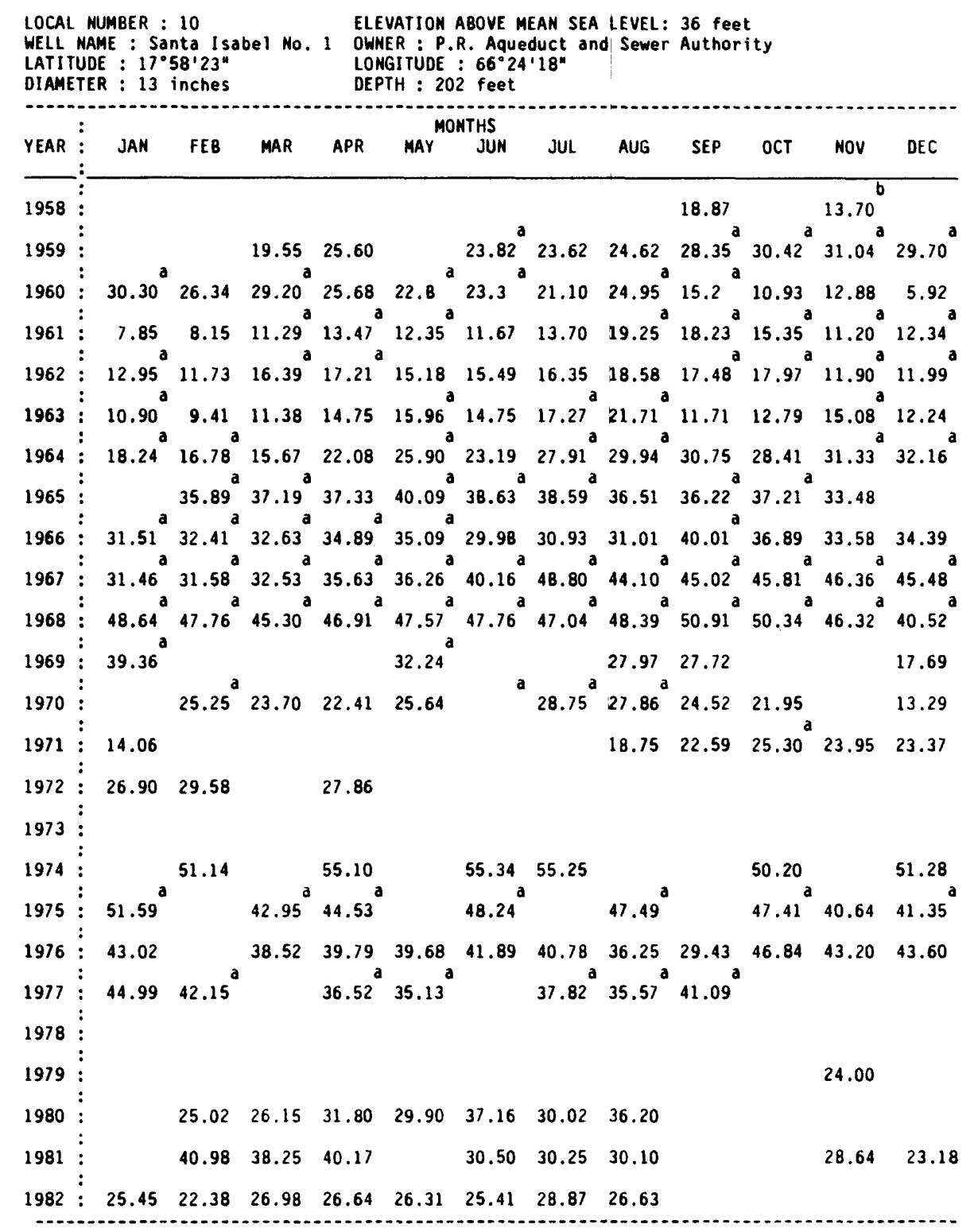

a punping, b recently pumped, e estimated, $h$ tape measurement, $j$ lowest water level recorded, n nearby punping well 
LOCAL NUMBER : 11

ELEVATION ABOVE MEAN SEA LEVEL: 36 feet

WELL NAME : Santa I sabel No. 2 OWNER: P.R. Aqueduct and Sewer Authority

LATITUOE: $17^{\circ} 58^{\prime} 23^{\prime \prime}$

DIAMETER : 13 inches

LONGITUDE : $66^{\circ} 24^{\prime} 17^{\prime \prime}$

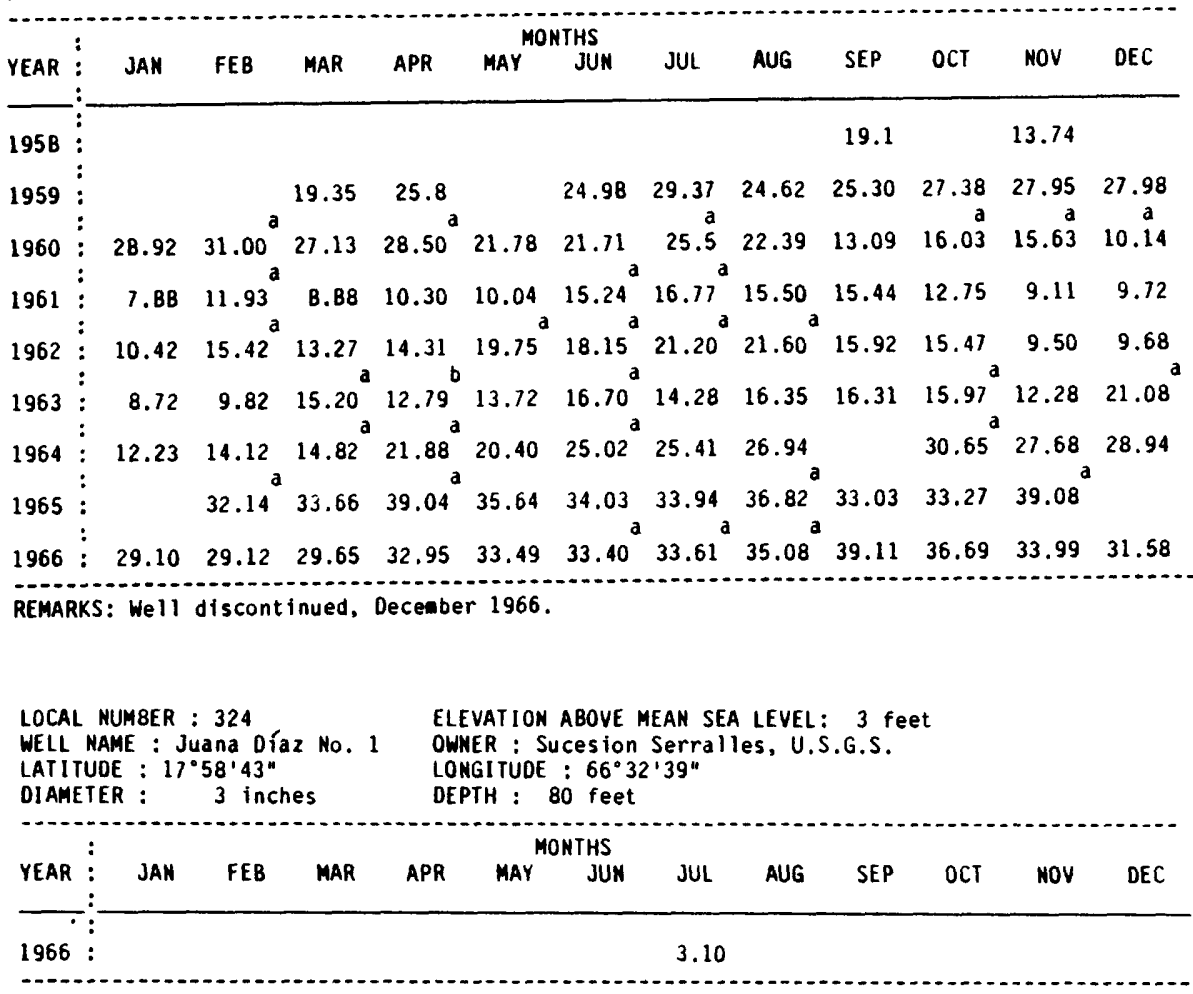

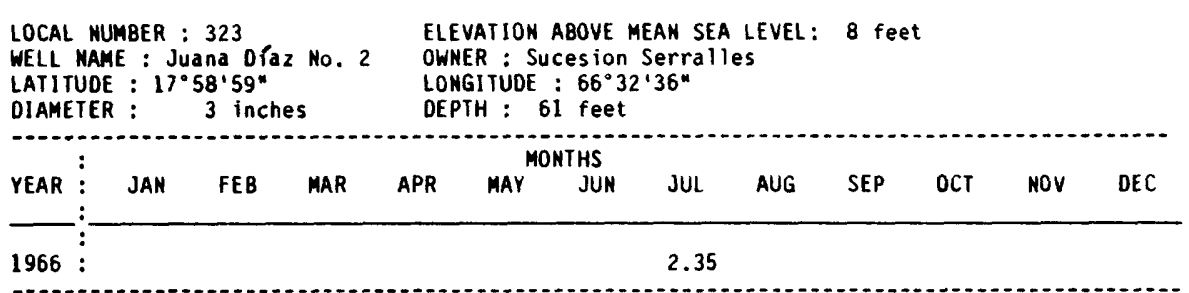

a pumping, b recently pumped, e estimated, h tape measurement, j lowest water level recorded, n nearby pumping well 


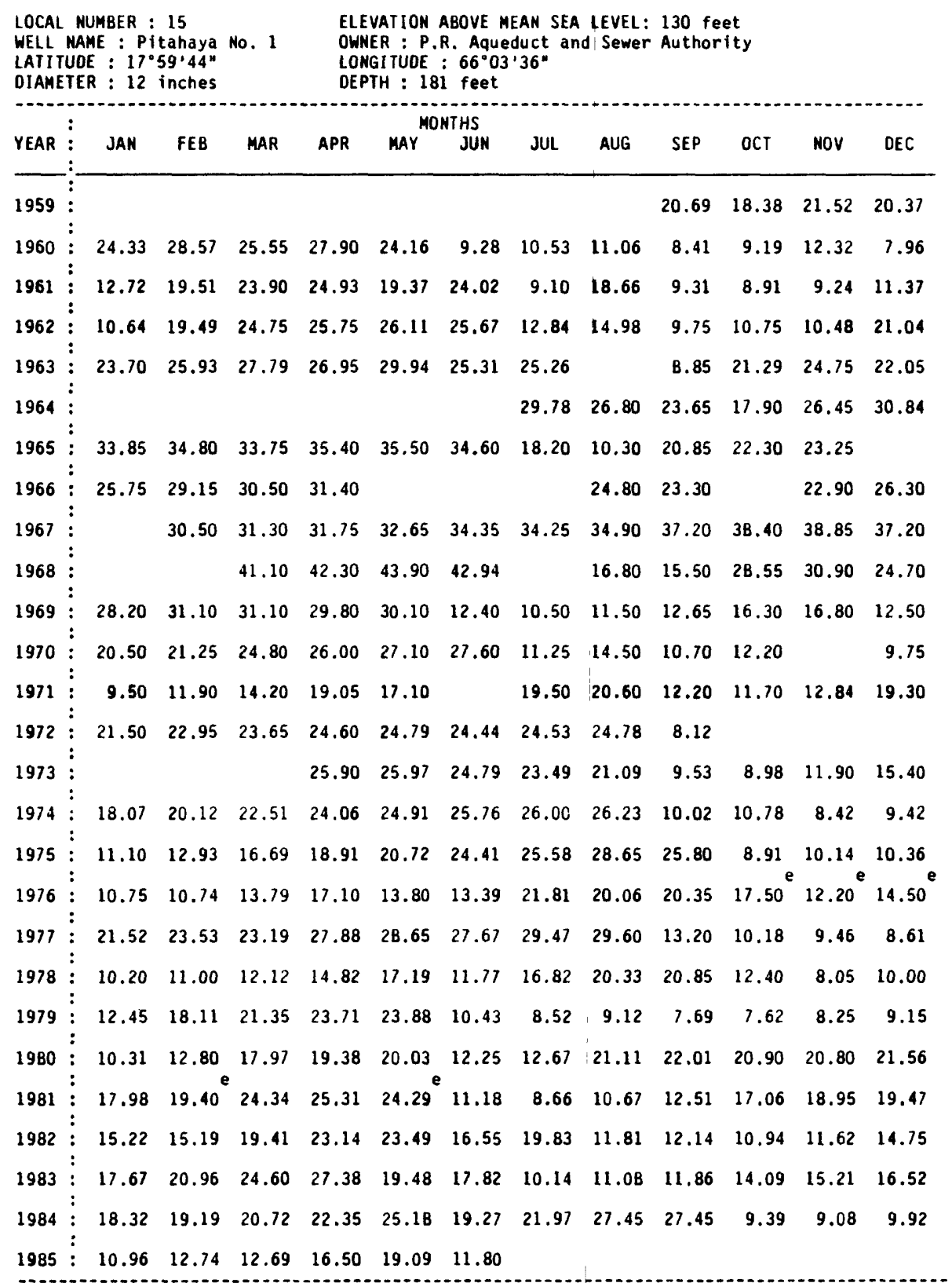

REMARKS: Well discont inued, July 1985.

a pumping, b recently pumped, estimated, $h$ tape measurement, $j$ lowest water level recorded,

n nearby pumping well 


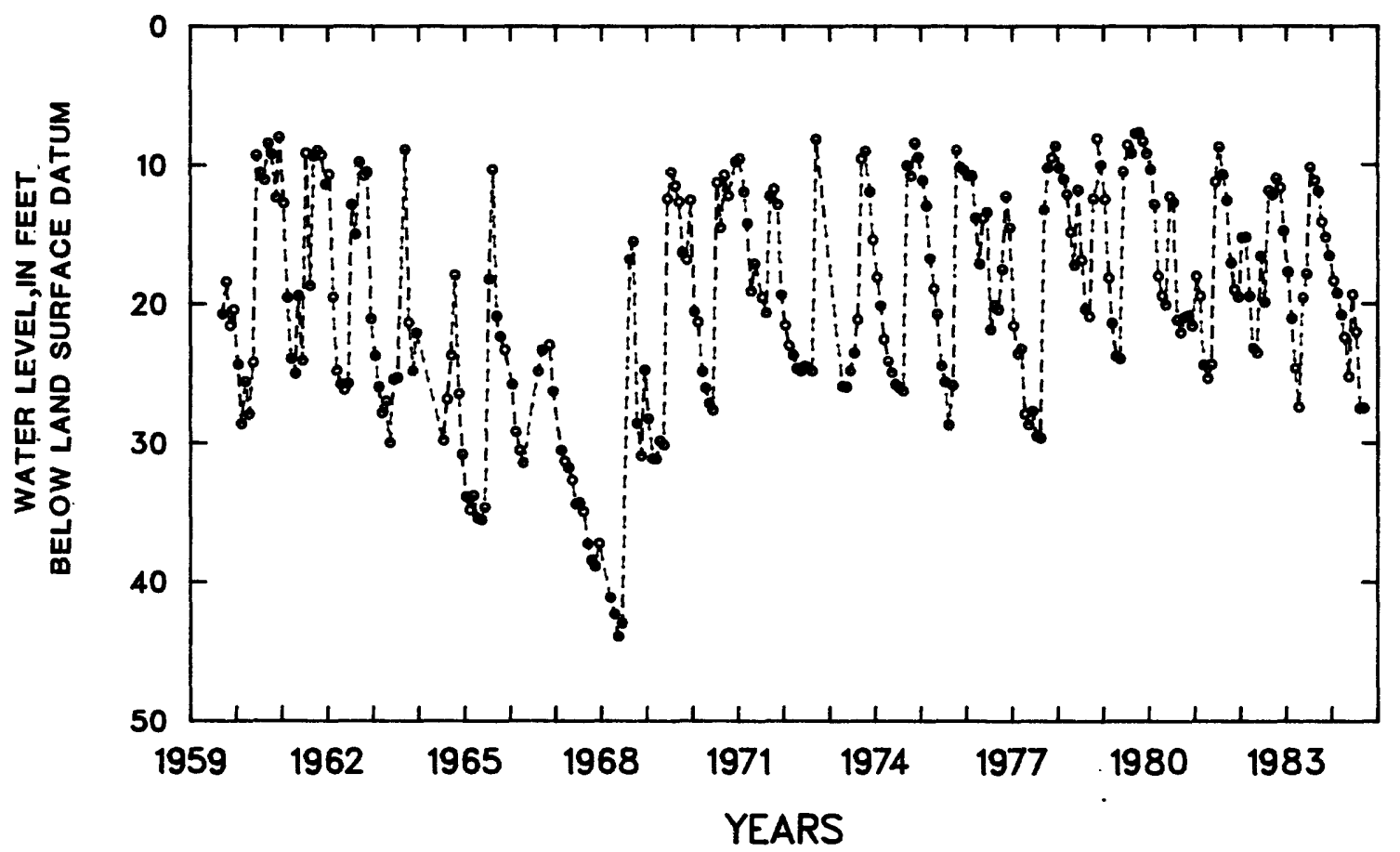

Figure 19.--Ground-water levels at Pltahaya Number 1, local number 15. 


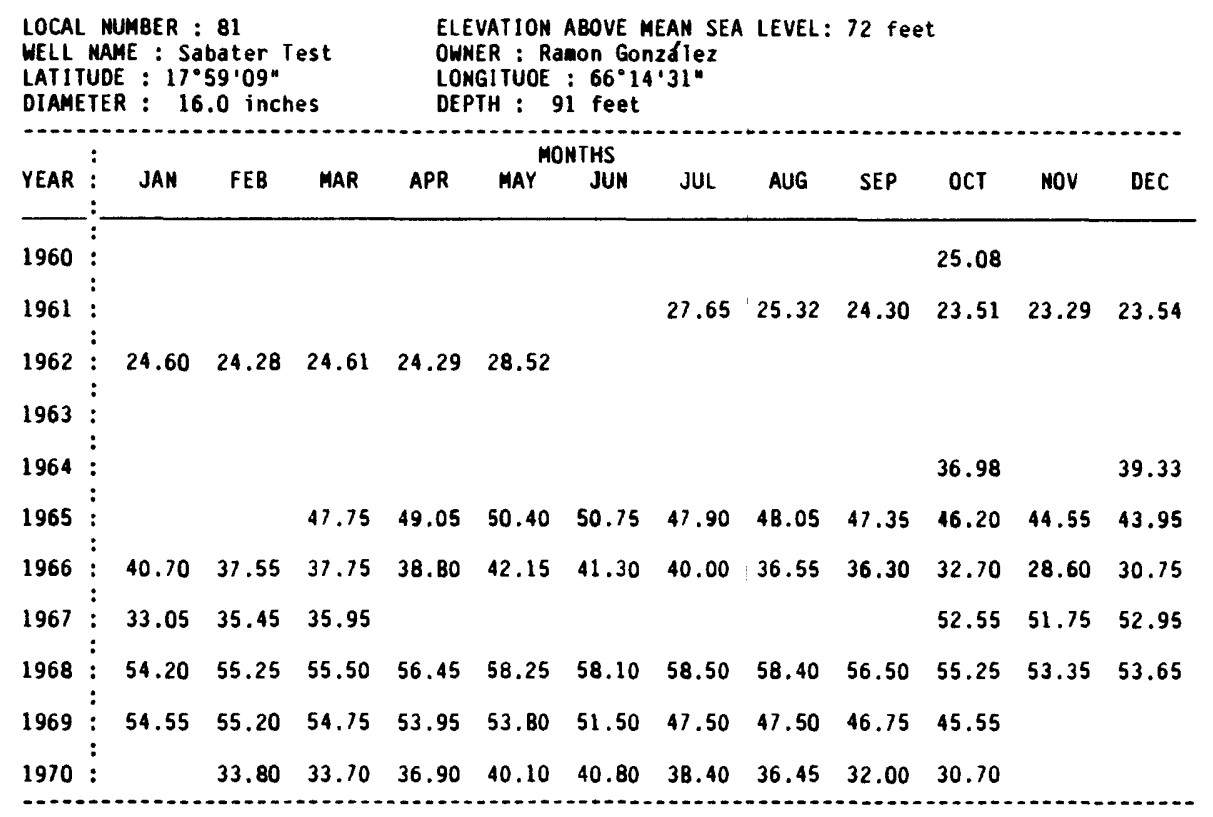

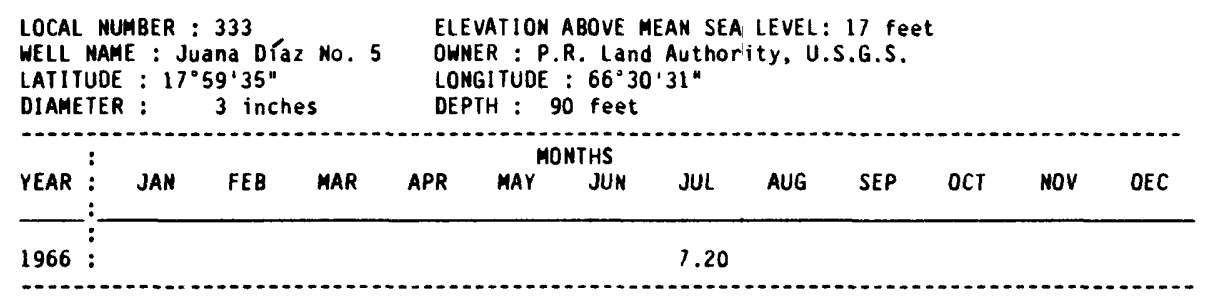

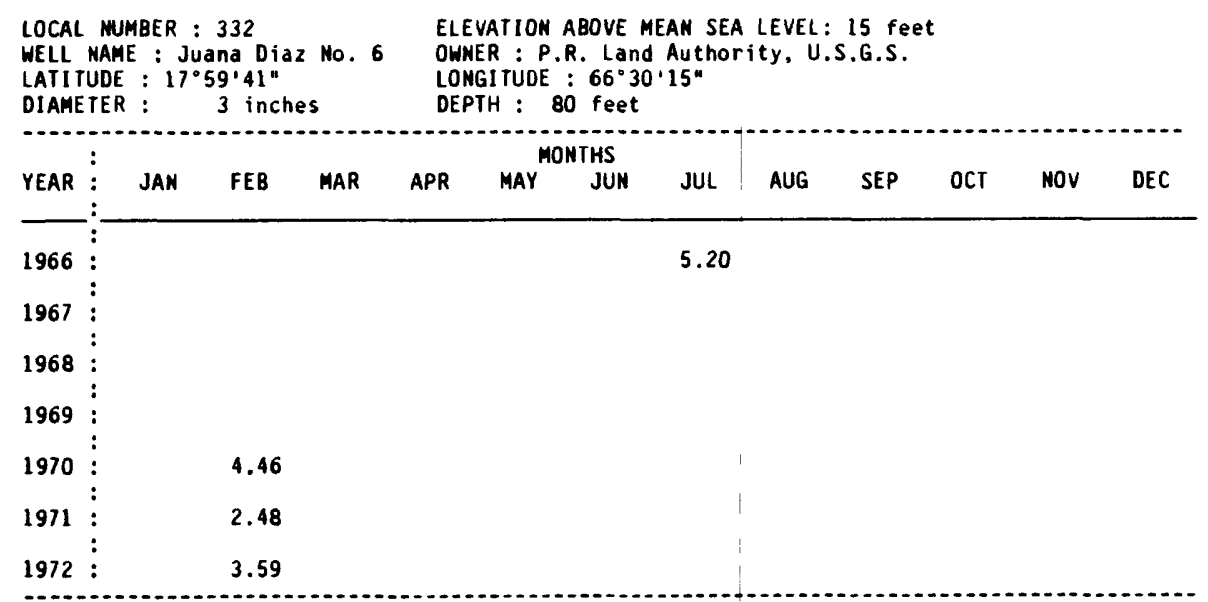

a pumping, b recently pumped, e estimated, $h$ tape measurement, $j$ lowest water level recorded, n nearby pumping well 


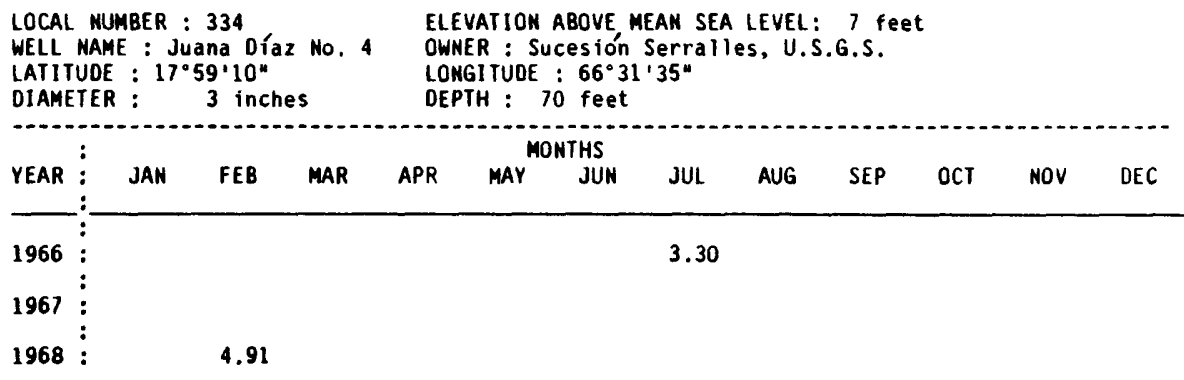

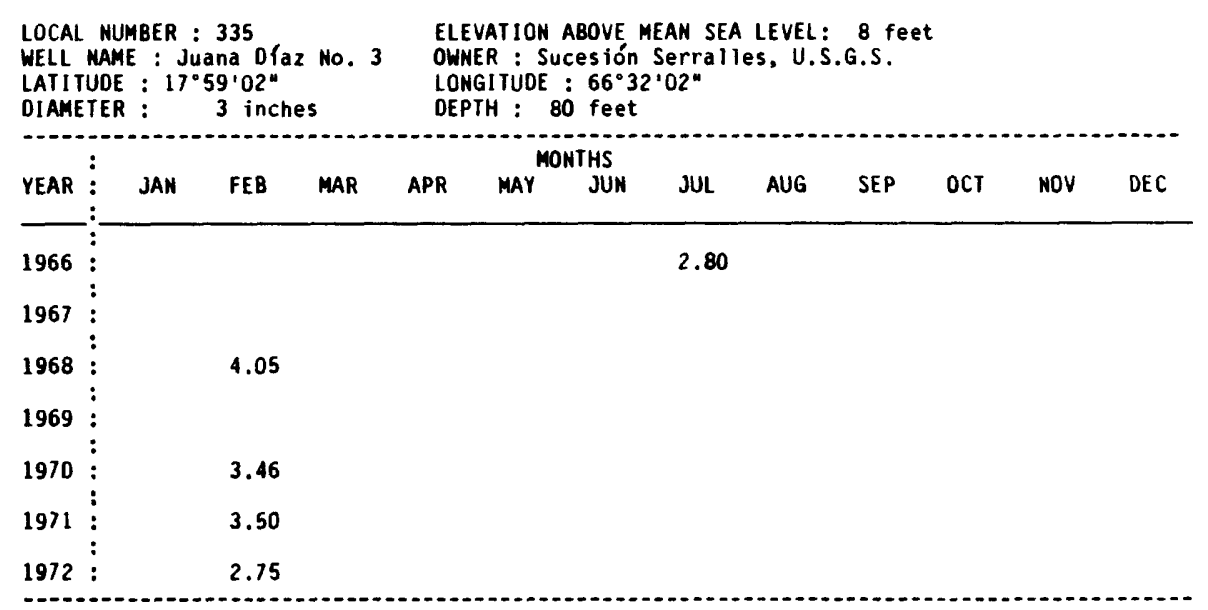

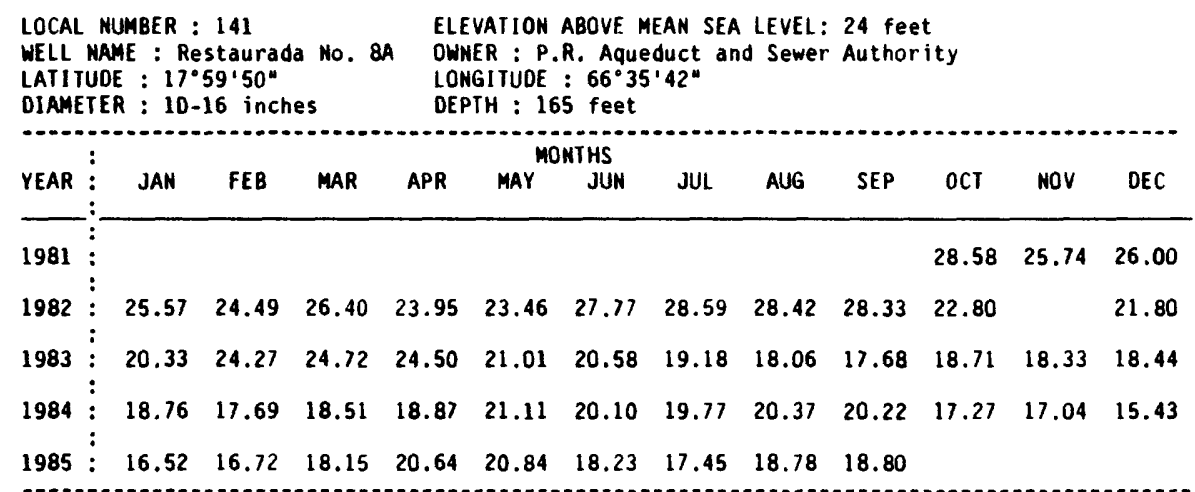

a pumping, b recently pumped, e estimated, $h$ tape measurement, $j$ lowest water level recorded, n nearby pumping well 
WELLS IN THE

SOUTH COAST PROVINCE

Tallaboa-Guayanilla-Yauco-Guánica

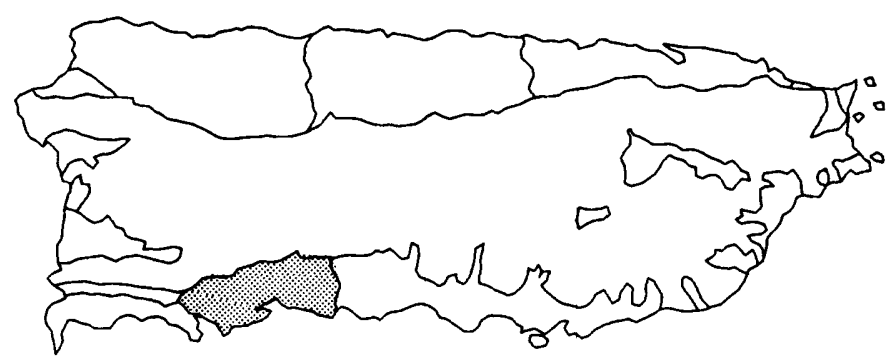

LOCAL NUMBER : 24

ELEVATION ABOVE MEAN SEA LEVEL: 24.B feet

WELL MAME : Tallaboa

LATITUDE : $18^{\circ} 00^{\prime} 51^{\prime \prime}$

DIAMETER : 12 inches

OWNER : P R W Wer Resources Authority

LONGITUDE : $66^{\circ} 43^{\prime} 55^{\prime \prime}$ DEPTH : 73 feet

\begin{tabular}{|c|c|c|c|c|c|c|c|c|c|c|c|c|}
\hline YEAR & JAN & FEB & MAR & APR & MAY ${ }^{\text {MO }}$ & $\begin{array}{l}\text { NTHS } \\
\text { JUN }\end{array}$ & JUL & AUG & SEP & OCT & NOV & DEC \\
\hline & 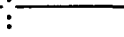 & & $a$ & & a & a & a & $a$ & $a$ & & a & \\
\hline 1959 & : & 4.89 & 35.20 & 6.66 & $16.80^{\circ}$ & 12.20 & 10.90 & $6.40^{\circ}$ & $5.40^{\circ}$ & 2.24 & 8.34 & 10.47 \\
\hline & a & a & & & & & & & & & & \\
\hline 1960 & $: 12.20$ & 10.40 & 7.63 & 5.88 & 4.73 & 11.54 & B. 23 & 3.56 & 10.08 & 9.83 & 3.75 & 4.59 \\
\hline & : & & & & & & & & a & & a & $a^{a}$ \\
\hline 1961 & 4.79 & 10.46 & 9.13 & 2.92 & 11.60 & 5.31 & 10.84 & 11.74 & 11.00 & 9.25 & 9.63 & 10.35 \\
\hline & a & & & & & & & a & a & & a & 2 \\
\hline 1962 & 8.40 & 12.48 & 10.65 & 2.75 & 11.04 & 11.90 & 11.63 & 7.48 & 12.63 & 16.22 & 12.28 & 8.65 \\
\hline & : & & a & & & & & & & & 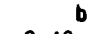 & \\
\hline 1963 & 6.51 & 8.76 & 26.39 & 14.90 & 5.54 & 5.74 & 7.32 & 9.27 & $7: 14$ & 7.18 & 9.42 & 9.24 \\
\hline 1964 & 9.76 & 9.46 & 9.56 & $18.80^{a}$ & $16.10^{\mathrm{a}}$ & $15.30^{\mathrm{a}}$ & 11.58 & 11.60 & 11.15 & $13.10^{a}$ & 11.70 & \\
\hline & a & a & & a & & & & & & & & \\
\hline 1965 & $: 24.00$ & 19.10 & 19.51 & 45.00 & 17.08 & 8.49 & 9.93 & 6.07 & 6.55 & 10.43 & 6.89 & \\
\hline 1966 & $: \quad 10.75$ & & 8.73 & 9.95 & 11.63 & 12.31 & 11.41 & 9.27 & & & & \\
\hline 1967 & 8.03 & 10.28 & & & 12.41 & & 17.81 & 18.20 & 9.04 & B.99 & 9.15 & 9.00 \\
\hline 1968 & 9.42 & 10.76 & 11.58 & & 13.83 & 12.58 & 9.30 & 8.06 & & & & \\
\hline
\end{tabular}

REMARKS: Well discont inued, Septeaber 1968.

a puaping, b recently punped, e estinated, $h$ tape neasurenent, $j$ lowest water level recorded,

n nearby puaping well 


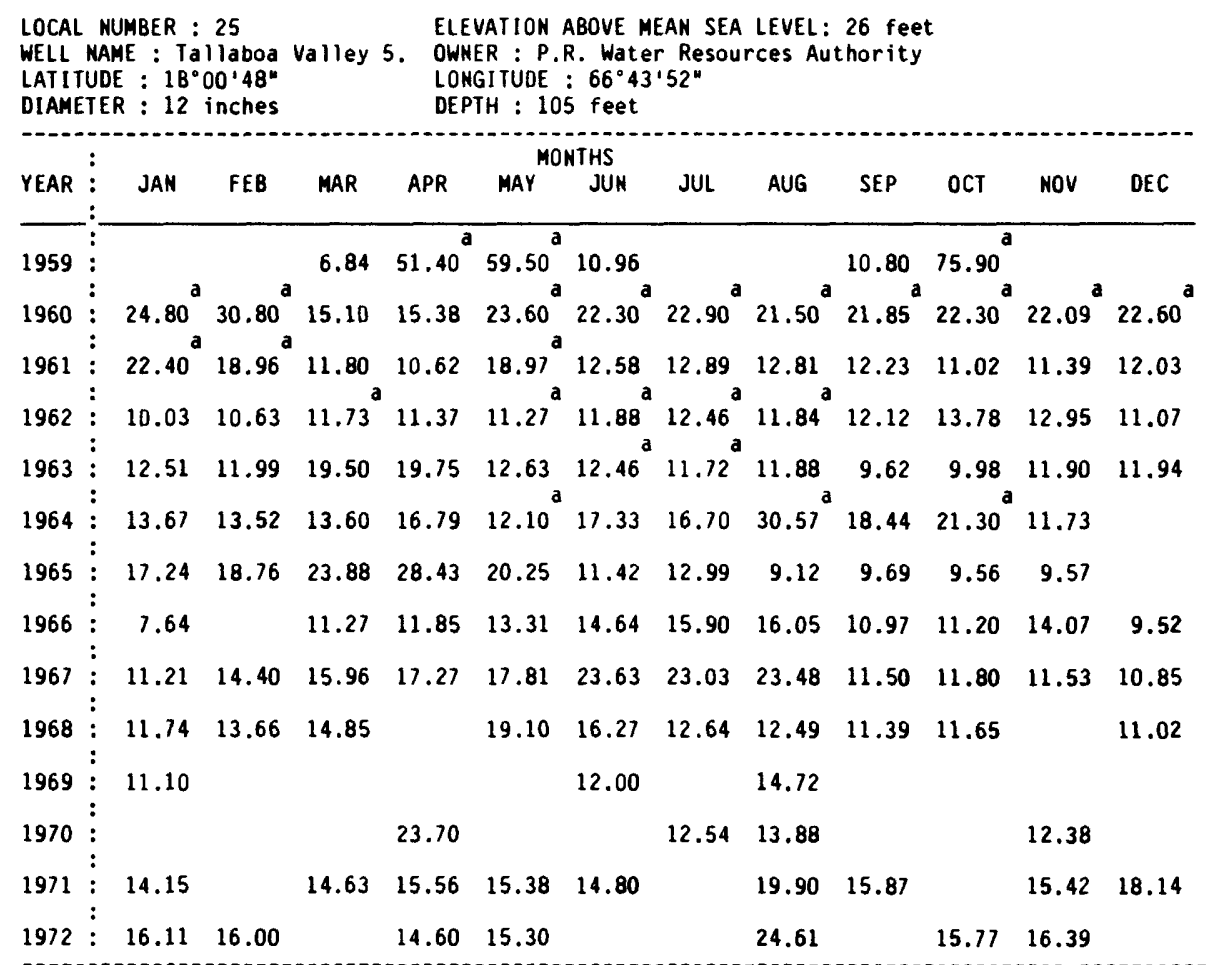

REMARKS: Well discont inued, Hovenber 1972.

a pumping, b recently punped, e estimated, h tape measurement, j lowest water level recorded. n nearby punping well 


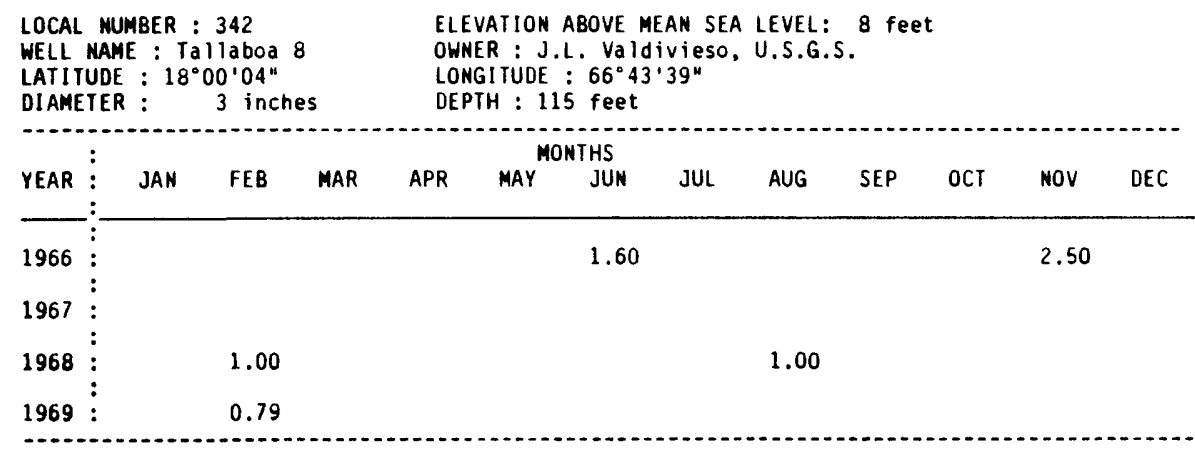

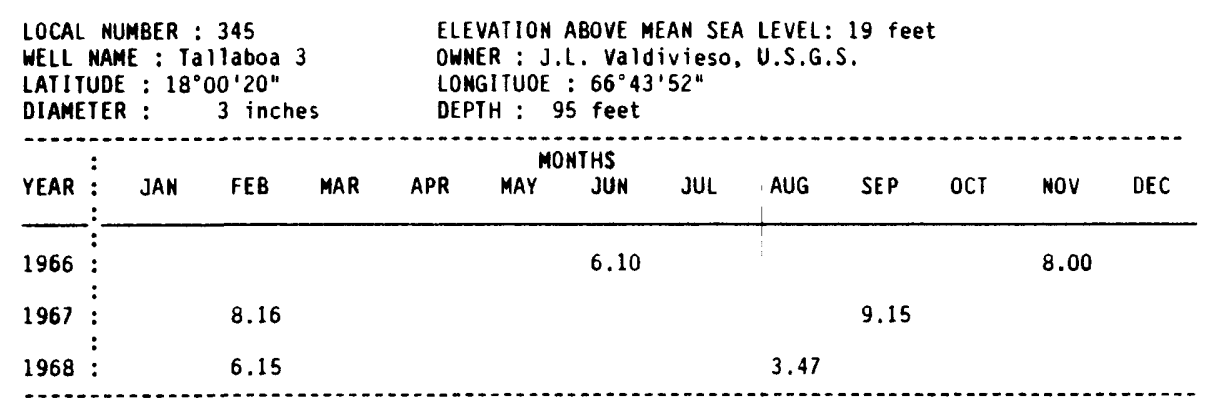

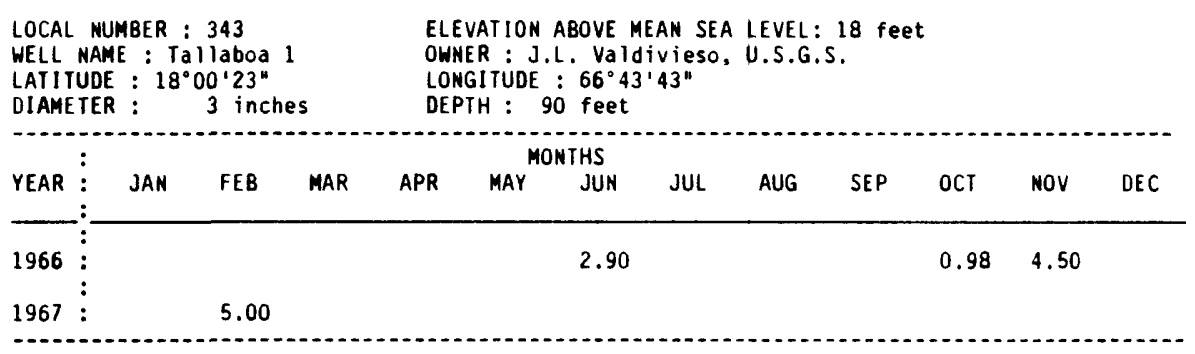

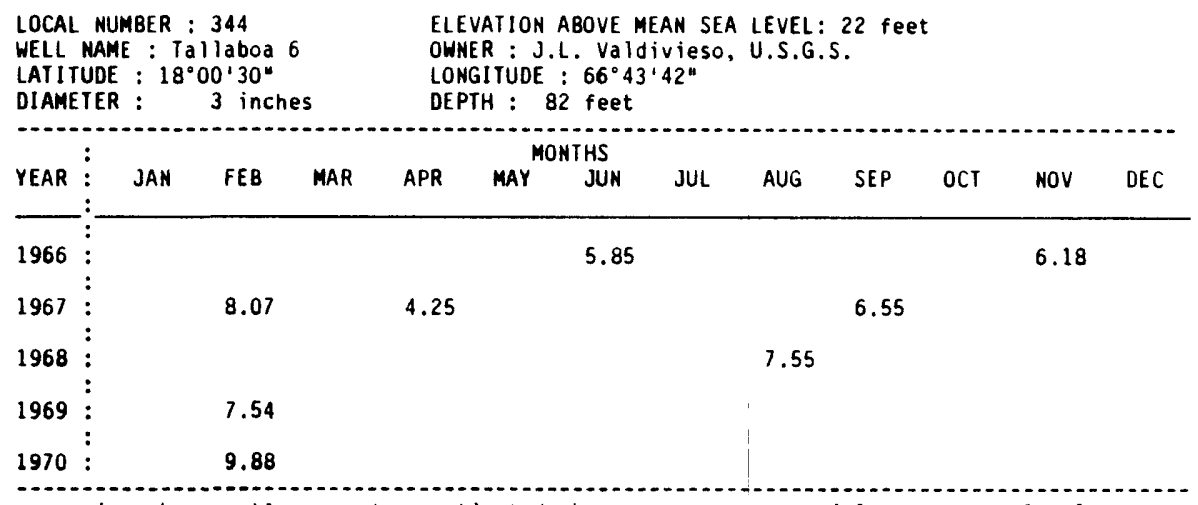

a puaping, b recently pumped, e estimated, $h$ tape measurement, $j$ lowest water level recorded, n nearby pumping well 


\begin{tabular}{|c|c|c|c|c|c|c|c|c|c|c|c|c|}
\hline $\begin{array}{l}\text { LOCAL } \\
\text { WELL } \\
\text { LATI } \\
\text { DIAME }\end{array}$ & $\begin{array}{l}\text { NUMBER } \\
\text { NAME : } \\
\text { UDE : } 18 \\
\text { TER : }\end{array}$ & $\begin{array}{l}348 \\
1 \text { laboa } \\
0 \text { ' } 32^{\prime \prime} \\
3 \text { inc }\end{array}$ & & & $\begin{array}{l}\text { ATIOI } \\
\text { R : } \\
\text { ITUDE } \\
\text { H : }\end{array}$ & $\begin{array}{l}\text { ABOVE } \\
\text { R. Dep } \\
66^{\circ} 4 \\
\text { feet }\end{array}$ & $\begin{array}{l}\text { IN SE } \\
\text { tment } \\
\text { lo" }\end{array}$ & $\begin{array}{l}\text { LEVE } \\
\text { f } \mathrm{Pu}\end{array}$ & $\begin{array}{l}6 \mathrm{fe} \\
=\text { Wor }\end{array}$ & U & & \\
\hline & : & & & & & NTHS & & & & & & \\
\hline YEAR & JAN & FEB & MAR & APR & MAY & JUN & JUL & AUG & SEP & OCT & NOV & DEC \\
\hline 1966 & : & & & & & 13.43 & & & & & 12.70 & \\
\hline 1967 & $\vdots$ & 2.35 & & & & & & & 4.80 & & & \\
\hline 1968 & : & 12.43 & & & & & & 9.46 & & & & \\
\hline 1969 & $\vdots$ & 11.45 & & & & & & & & & & \\
\hline 1970 & $\vdots$ & 15.20 & & & & & & & & & & \\
\hline
\end{tabular}

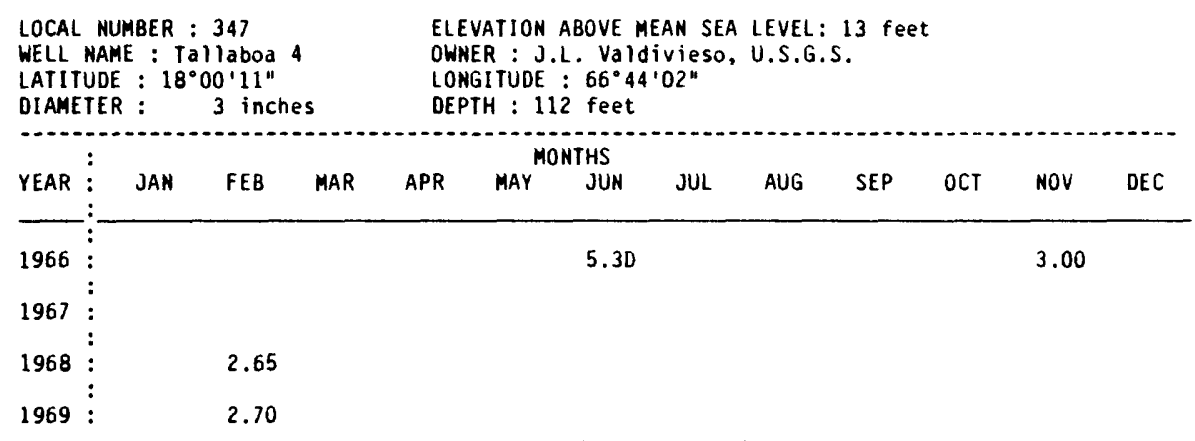

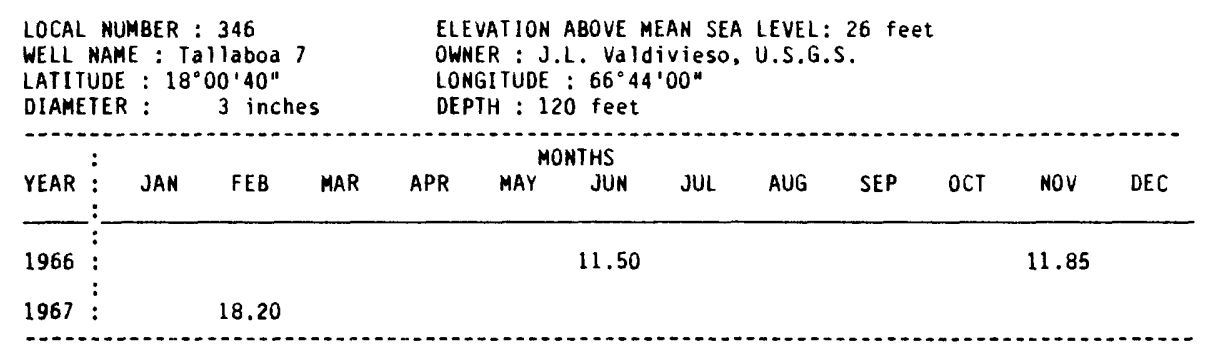

\begin{tabular}{|c|c|c|c|c|c|c|c|c|c|c|c|c|}
\hline $\begin{array}{l}\text { LOCA } \\
\text { WELL } \\
\text { LAT I } \\
\text { DIAM }\end{array}$ & $\begin{array}{l}\text { L MUMBER } \\
\text { NAME : } \\
\text { TUDE : } 18 \\
\text { ETER : }\end{array}$ & $\begin{array}{l}349 \\
\text { laboa } \\
0^{\prime} 03^{\prime \prime} \\
3 \text { inc }\end{array}$ & & $\begin{array}{l}E L \\
\text { OW } \\
\text { LO } \\
\text { DE }\end{array}$ & $\begin{array}{l}\text { ATION } \\
R: U \\
\text { ITUDE } \\
H: 1\end{array}$ & $\begin{array}{l}\text { BOVE } \\
\text { on Ca } \\
66^{\circ} 4 \\
\text { feet }\end{array}$ & $\begin{array}{l}\text { AN SE } \\
\text { ide, } \\
17 "\end{array}$ & $\begin{array}{l}\text { LEVEL: } \\
\text { S.G.S. }\end{array}$ & $9 \mathrm{fe}$ & & & \\
\hline$\cdots$ & $:$ & & & & & THS & & & & $=$ & . & \\
\hline YEAR & JAN & FEB & MAR & APR & MAY & JUN & JUL & AUG & SEP & OCT & Nov & DEC \\
\hline 1966 & $\vdots$ & & & & & 3.50 & & & & & 5.20 & \\
\hline 1967 & : & 5.15 & & & & & & & 4.75 & & & \\
\hline $196 \mathrm{~B}$ & : & 3.65 & & & & & & 3.67 & & & & \\
\hline 1969 & : & 3.39 & & & & & & & & & & \\
\hline
\end{tabular}

a pumping, b recently pumped, e estimated, $h$ tape measurement, $j$ lowest water level recorded, $n$ nearby pumping well 


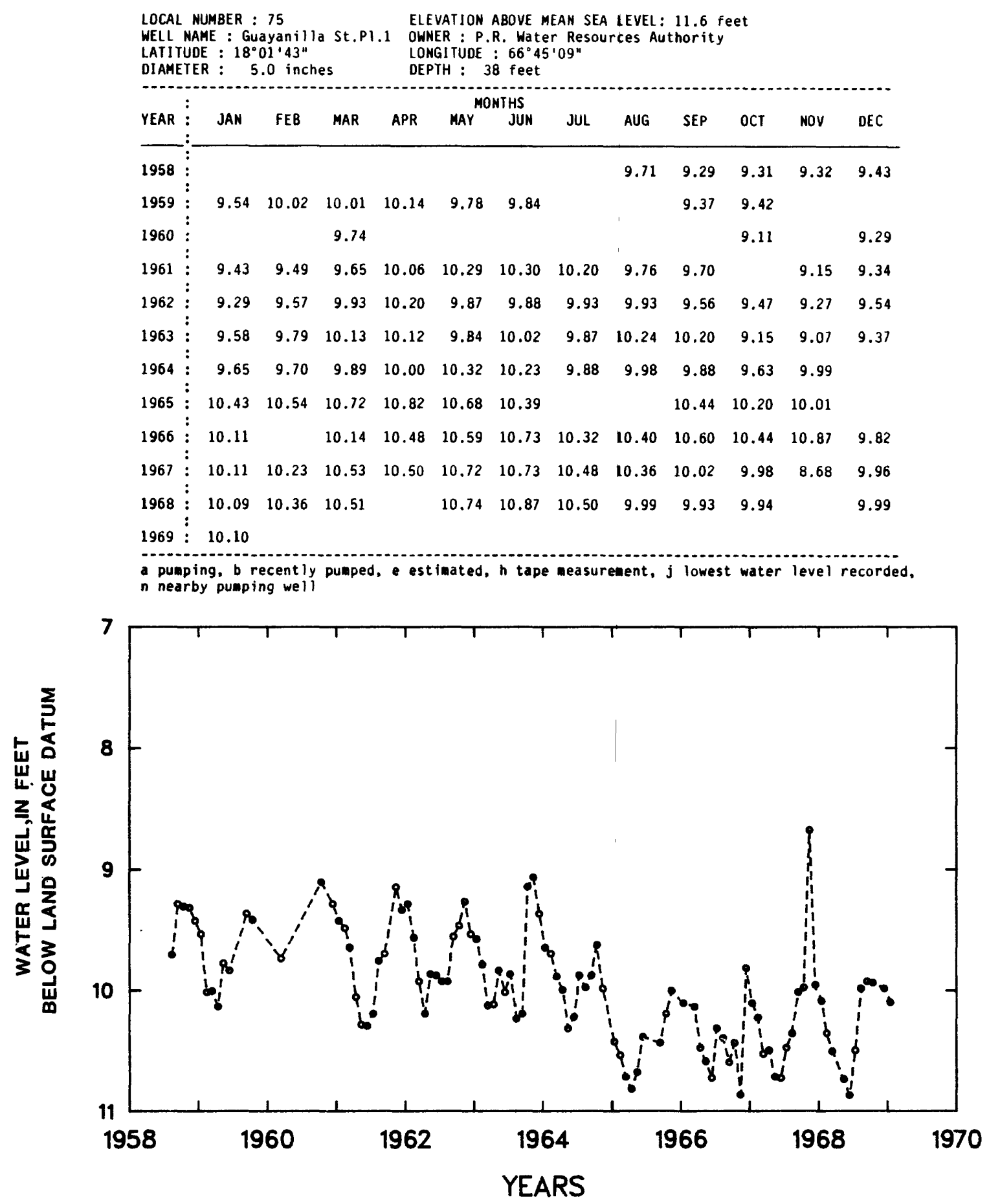

Figure 20.--Ground-water levels at Guayanilla Steam Plant 1. local number 75. 
LOCAL NUMBER : 350

WELL MAME : Guayanilla 6

LATITUDE : $18^{\circ} 00^{\prime} 35^{\prime \prime}$

DIAMETER :

3 inches

ELEVATION ABOVE MEAN SEA LEVEL: 3 feet

OWMER : Mario Mercado, U.S.G.S.

LONGITUDE : $66^{\circ} 45^{\prime} 57^{\prime \prime}$

DEPTH : 90 feet

\begin{tabular}{lllllll} 
YEAR & JAN FEB MAR APR MAY JONTHS & JUL AUG SEP OCT NOV OEC \\
\hline $1966:$ & & 2.12 & 1.75 \\
$1967:$ & & & \\
$1968:$ & & & \\
$1969:$ & 2.53 & & \\
$1970:$ & 2.30 & &
\end{tabular}

REMARK: Well found destroyed February, 1970.

LOCAL MUMBER : 353

WELL MAME : Guayanilla 7

LATITUDE : $18^{\circ} 00^{\prime} 54^{\prime \prime}$

DIAMETER : 3 inches

ELEVATION ABOVE MEAN SEA LEVEL: 15 feet

OWMER : Mario Mercado, U.S.G.S.

LONGITUDE : $66^{\circ} 45^{\prime} 58^{\prime \prime}$

OEPTH : 75 feet

\begin{tabular}{llllllll} 
YEAR & JAN FEB MAR APR MAY JUNTHS & JUL AUG & SEP & OCT & NOV & DEC \\
\hline $1966:$ & & 9.40 & 9.95 \\
1967 & & & & \\
1968 & & & & \\
1969 & 11.96 & & & \\
& Well destroyed.
\end{tabular}

REMARK: Well found destroyed February, 1969.

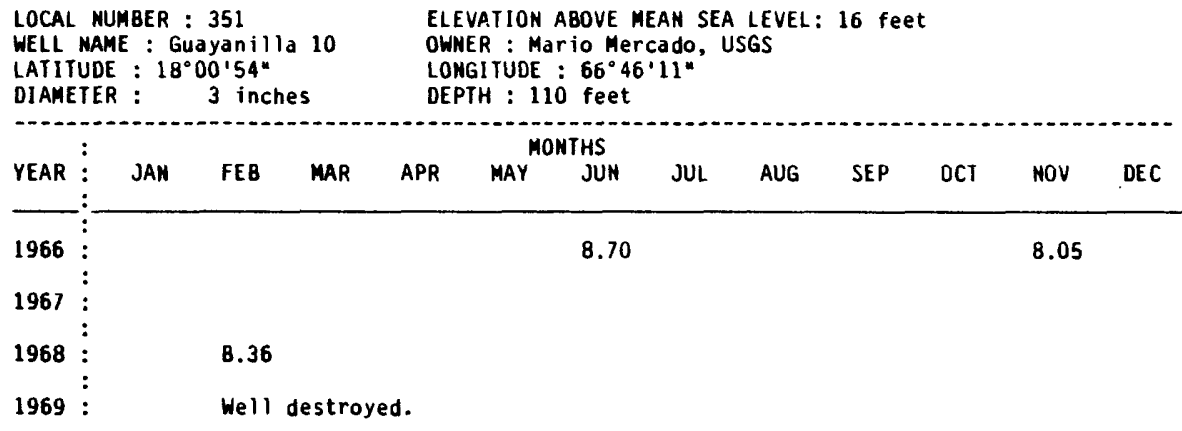

REMARK: Well found destroyed, February 1969

a puaping, b recently pumped, e estimated, $h$ tape measurement, $J$ lowest water level recorded, n nearby pumping well 
LOCAL NUMBER : 352 ELEVATION ABOVE MEAN SEA LEVEL: 7 feet

WELL NAME : Guayanilla 5. OWNER : P.R. Aqueduct and Sewer Authority

LATITUDE : $18^{\circ} 01^{\prime} 02^{\prime \prime}$

DIAMETER : 5 inches

LONGIT

\begin{tabular}{|c|c|c|c|c|c|c|c|c|c|c|c|c|}
\hline YEAR & : JAN & FEB & MAR & APR & MAY ${ }^{\text {MOI }}$ & $\begin{array}{l}\text { NTHS } \\
\text { JUN }\end{array}$ & JUL & AUG & SEP & $\mathrm{OCT}$ & NOV & DEC \\
\hline 1961 & : & & 8.61 & 9.85 & 8.19 & 9.76 & 9.63 & 9.40 & 7.39 & 3.86 & 3.50 & 3.71 \\
\hline 1962 & $3.41^{h}$ & & 11.53 & 13.12 & 13.56 & 17.38 & 12.55 & 9.63 & 7.04 & 6.16 & 4.01 & 5.95 \\
\hline 1963 & 6.86 & 9.96 & & & 15.12 & 12.77 & 11.80 & & & 3.66 & 3.67 & 3.36 \\
\hline 1964 & 4.99 & 10.96 & 12.68 & & 17.31 & 17.47 & 16.97 & 15.81 & & & & \\
\hline 1965 & : & & & 15.98 & 16.04 & & & 6.45 & 5.32 & 7.72 & 6.02 & 4.94 \\
\hline 1966 & 4.80 & 9.26 & 13.70 & 14.45 & & 15.30 & 14.40 & 14.65 & 11.80 & 9.80 & 6.87 & 6.39 \\
\hline 1967 & 7.52 & 12.18 & 15.88 & 16.17 & 16.40 & 16.25 & 16.30 & 14.95 & 13.90 & 13.10 & 7.45 & 7.65 \\
\hline 1968 & 7.15 & 1.45 & 1.50 & 10.50 & 10.70 & 9.00 & 1.52 & 1.42 & & & & \\
\hline
\end{tabular}

REMARKS: Station discont inued, September 1968, affected by nearby pumping.

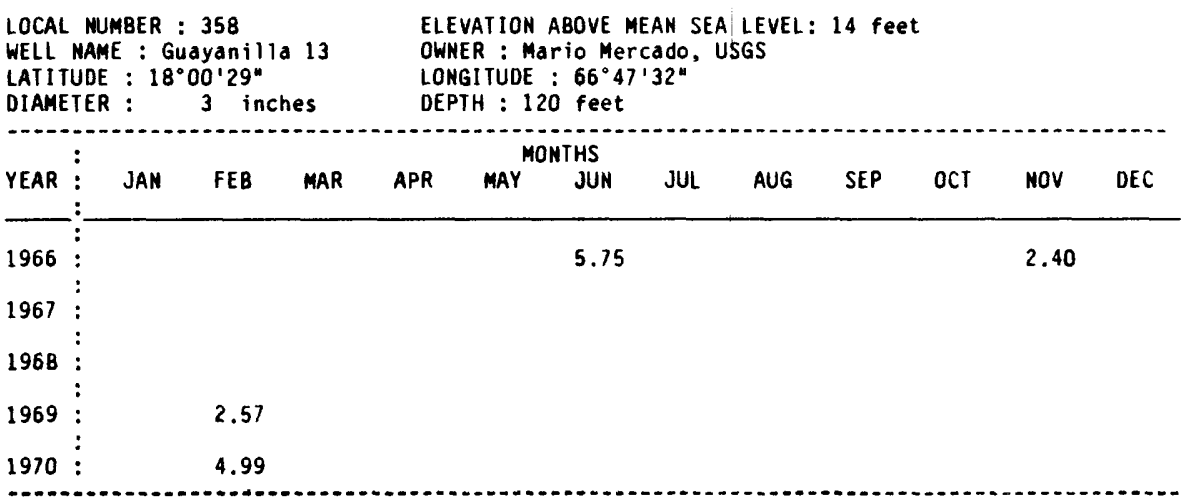

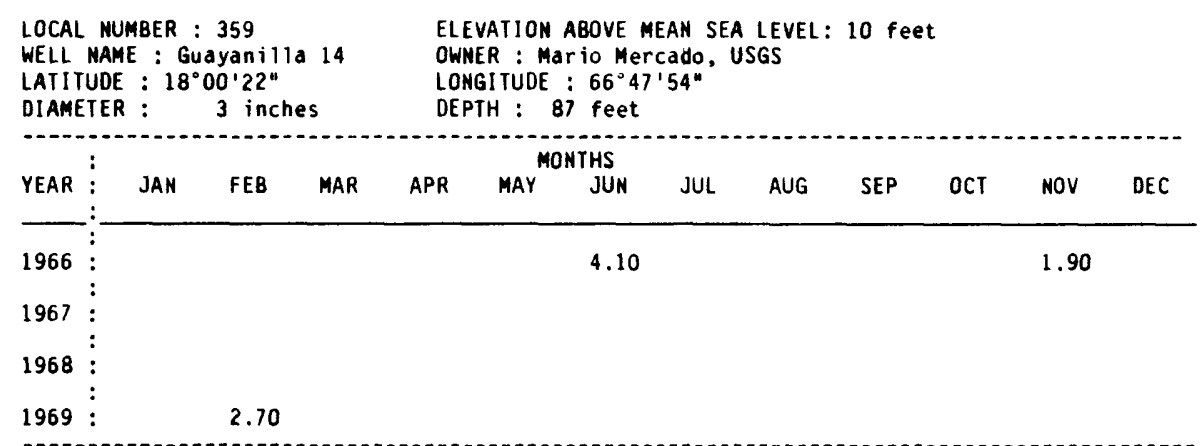

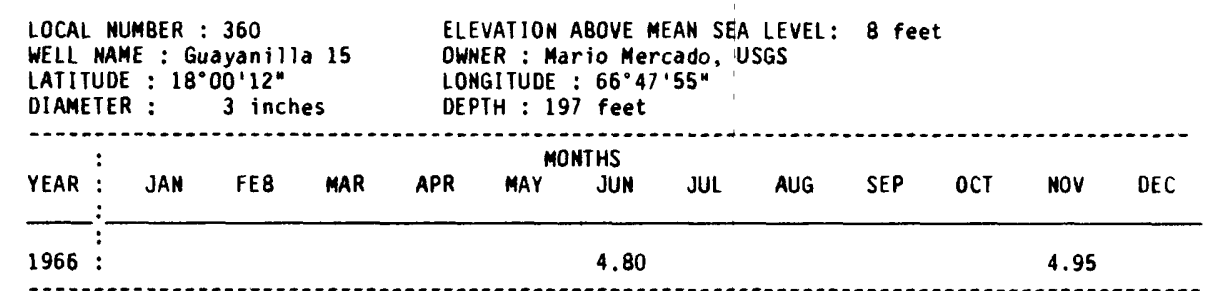

a pumping, b recently puaped, e estiaated, $h$ tape aeasurement, $j$ lowest water leve 1 recorded,

n nearby pumping well 


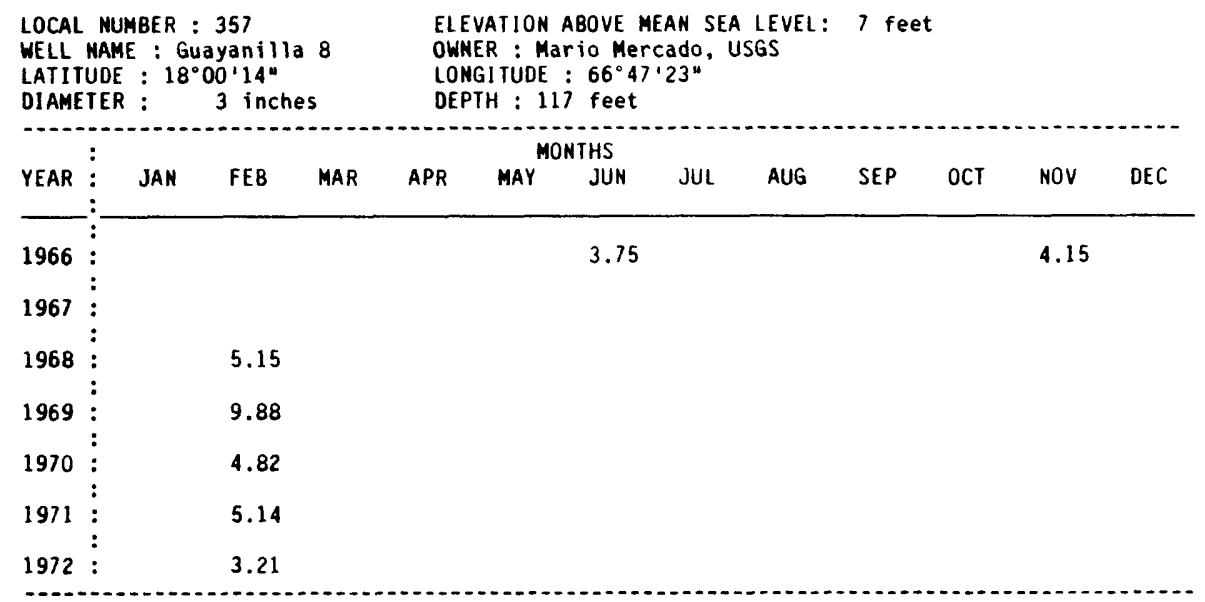

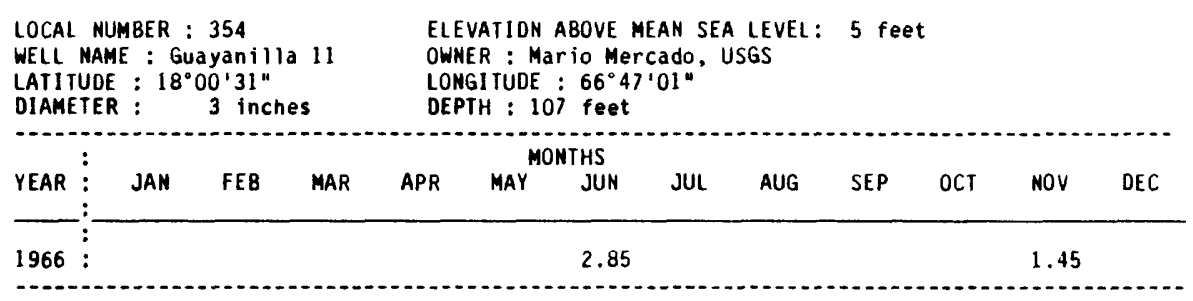

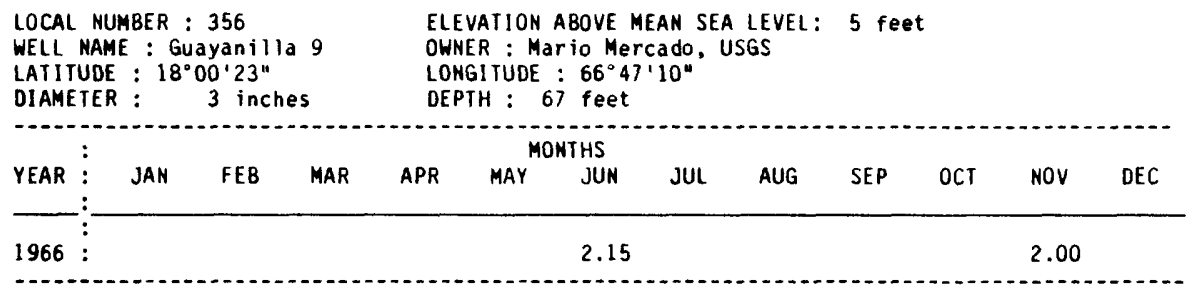

a pumping, b recently pumped, e estimated, $h$ tape measurement, $j$ lowest water level recorded, n nearby pumping well 


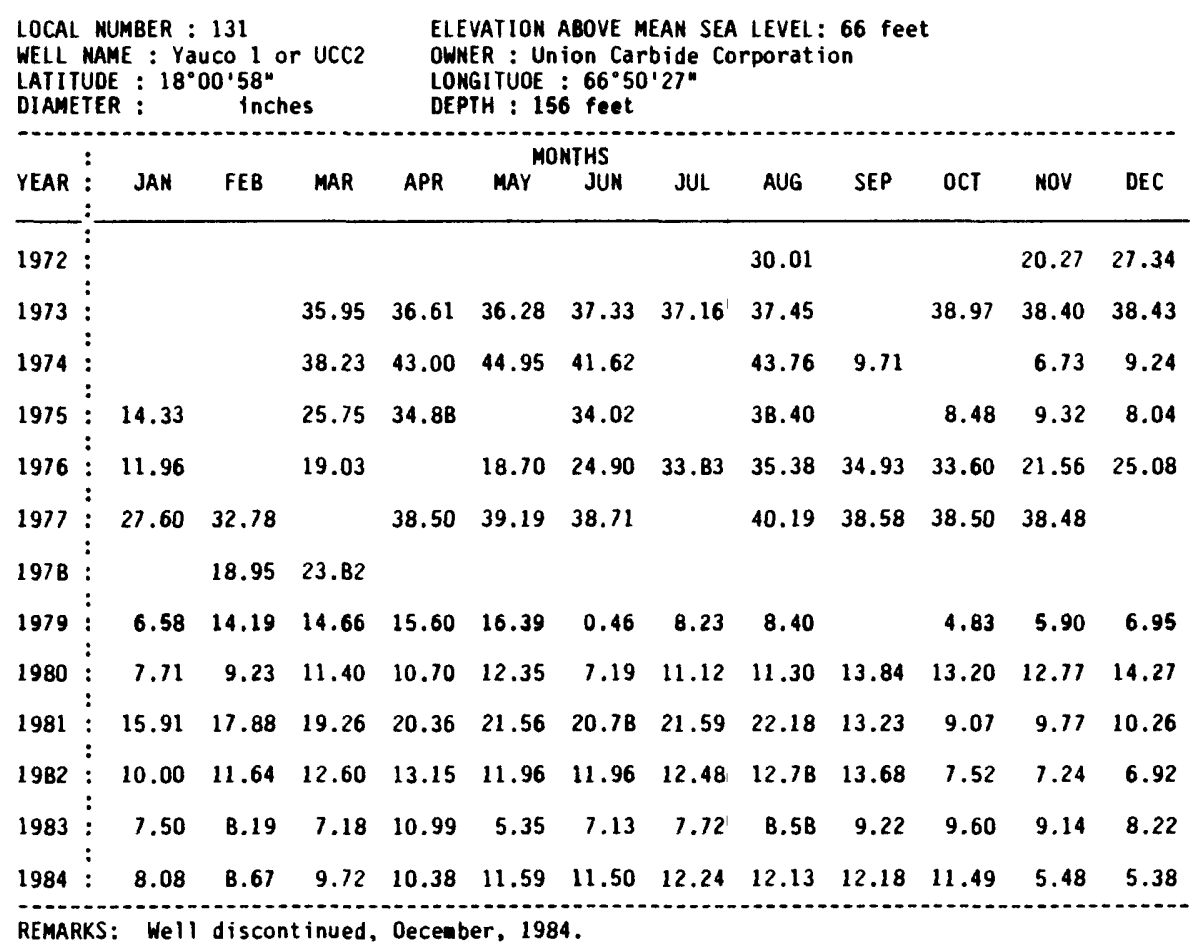

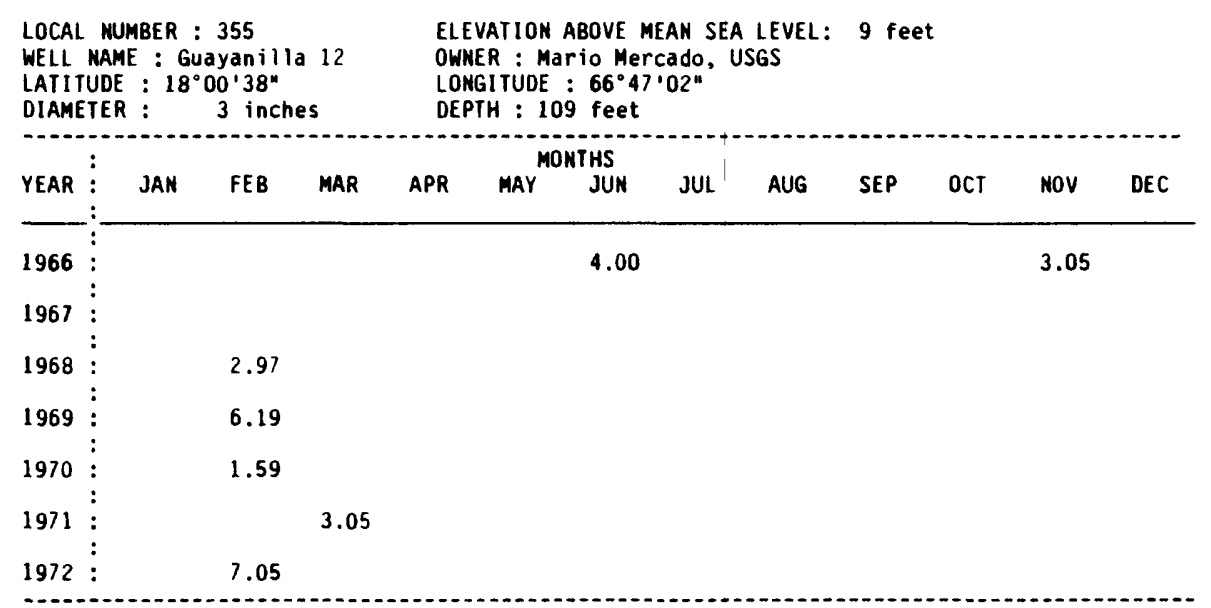

a pumping, b recently pumped, e estimated, $h$ tape measurement, $j$ lowest water level recorded, n nearby pumping well 


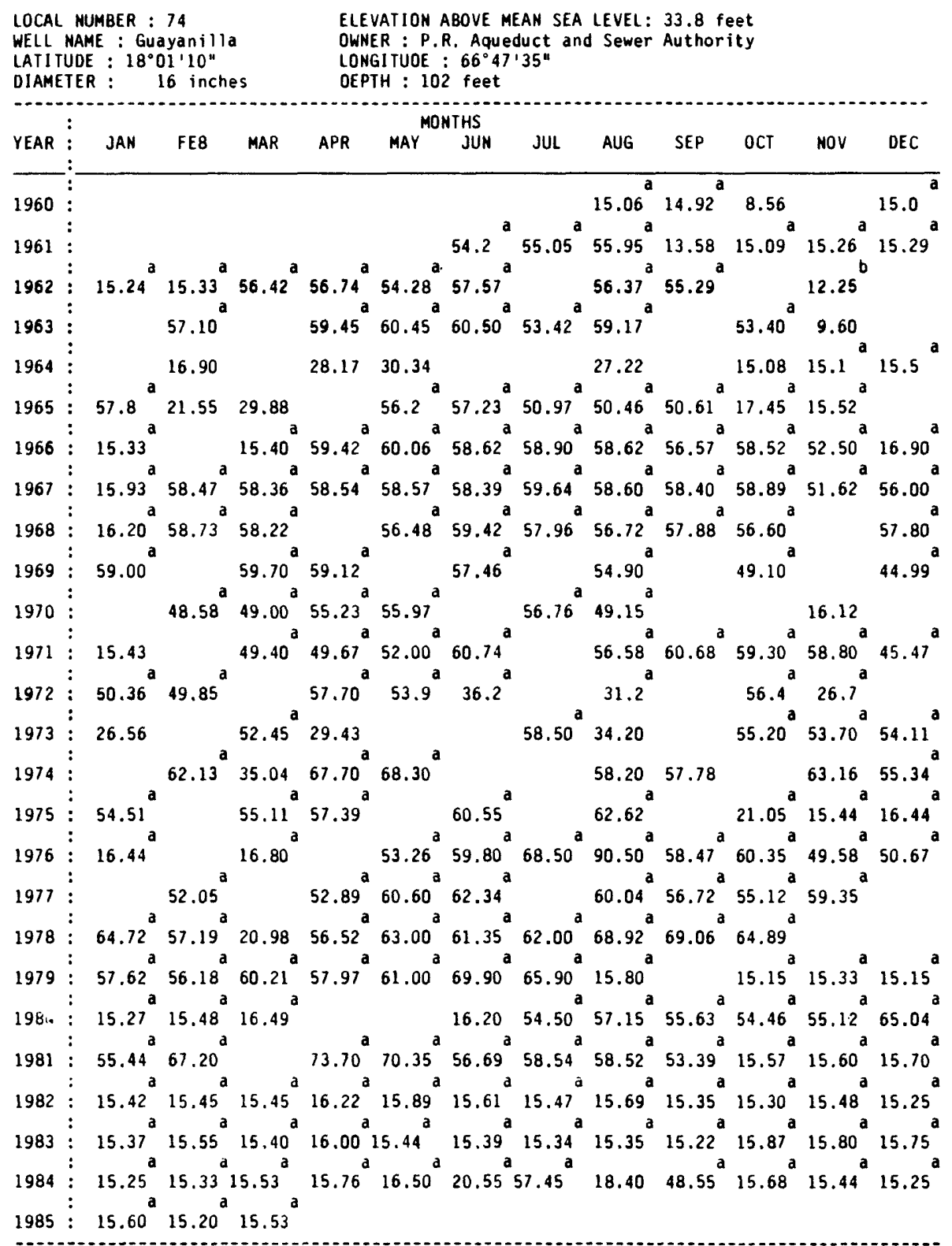

REMARKS: Well discont inued, April 1985.

a puaping, b recently pumped. e estiaated, $h$ tape measurement, $j$ lowest water level recorded, n nearby puaping well 


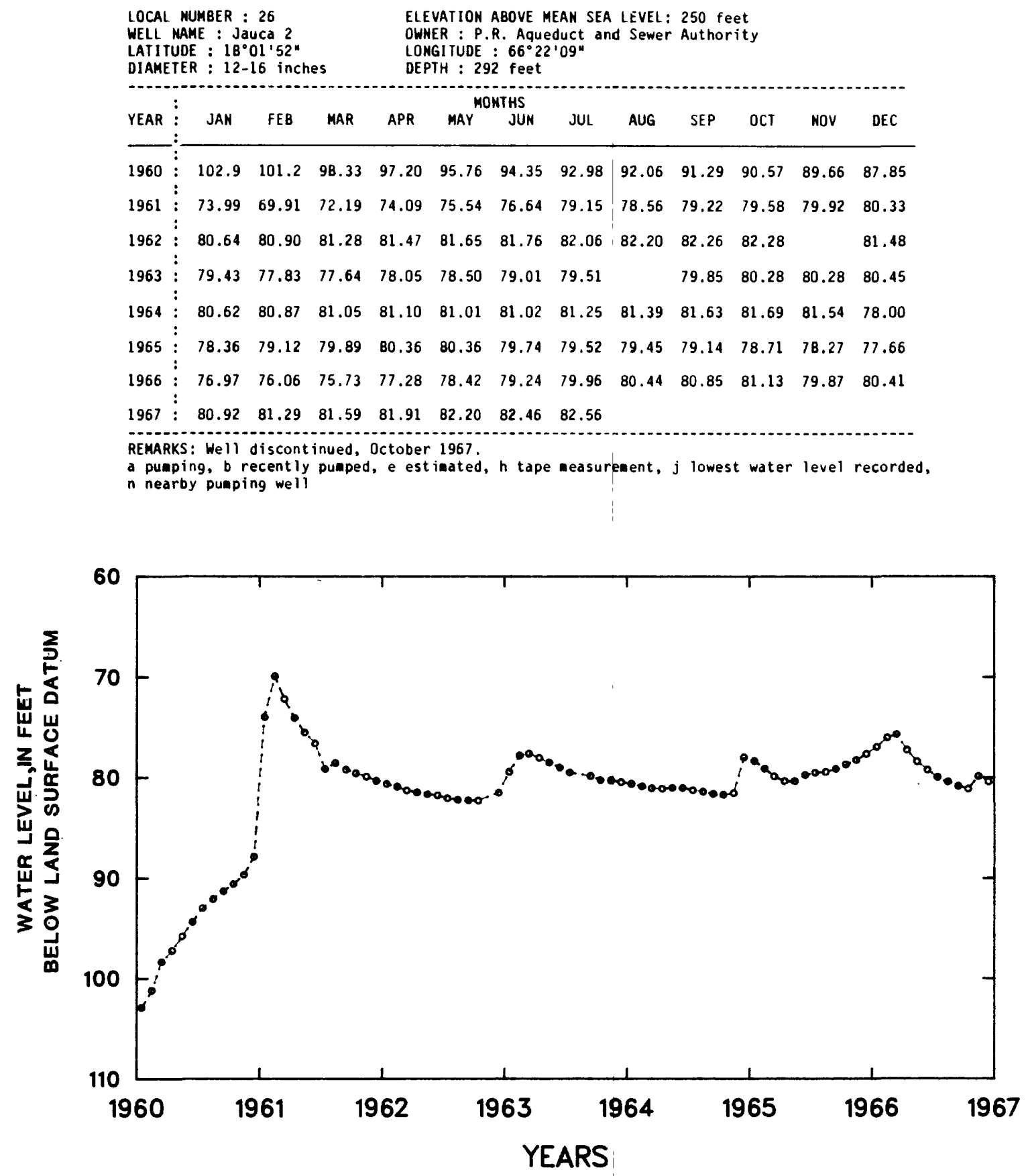

Figure 21.--Ground-water leveis at Jauca 2, locai number 26. 


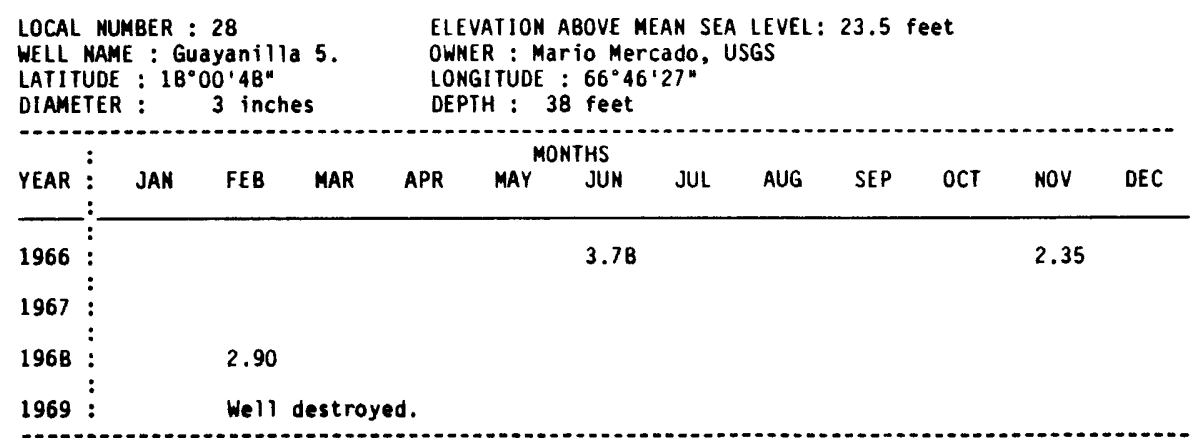

REMARKS: Well found destroyed $02 / 1969$.

a pumping, b recently pumped, e estimated, $h$ tape measurement, $j$ lowest water level recorded,

n nearby pumping well

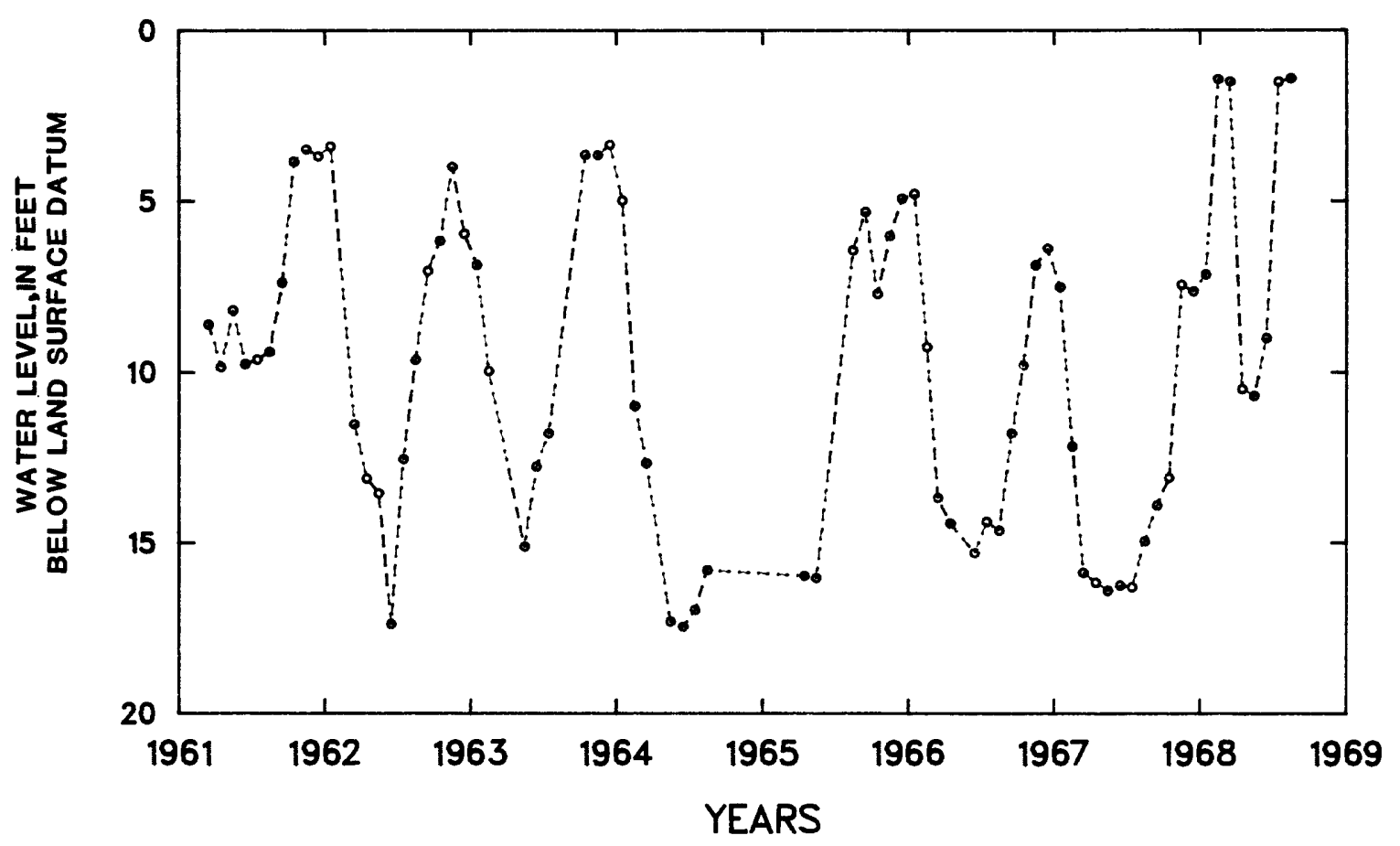

Figure 22.--Ground-water levels at Guayanilla 5, local number 28. 


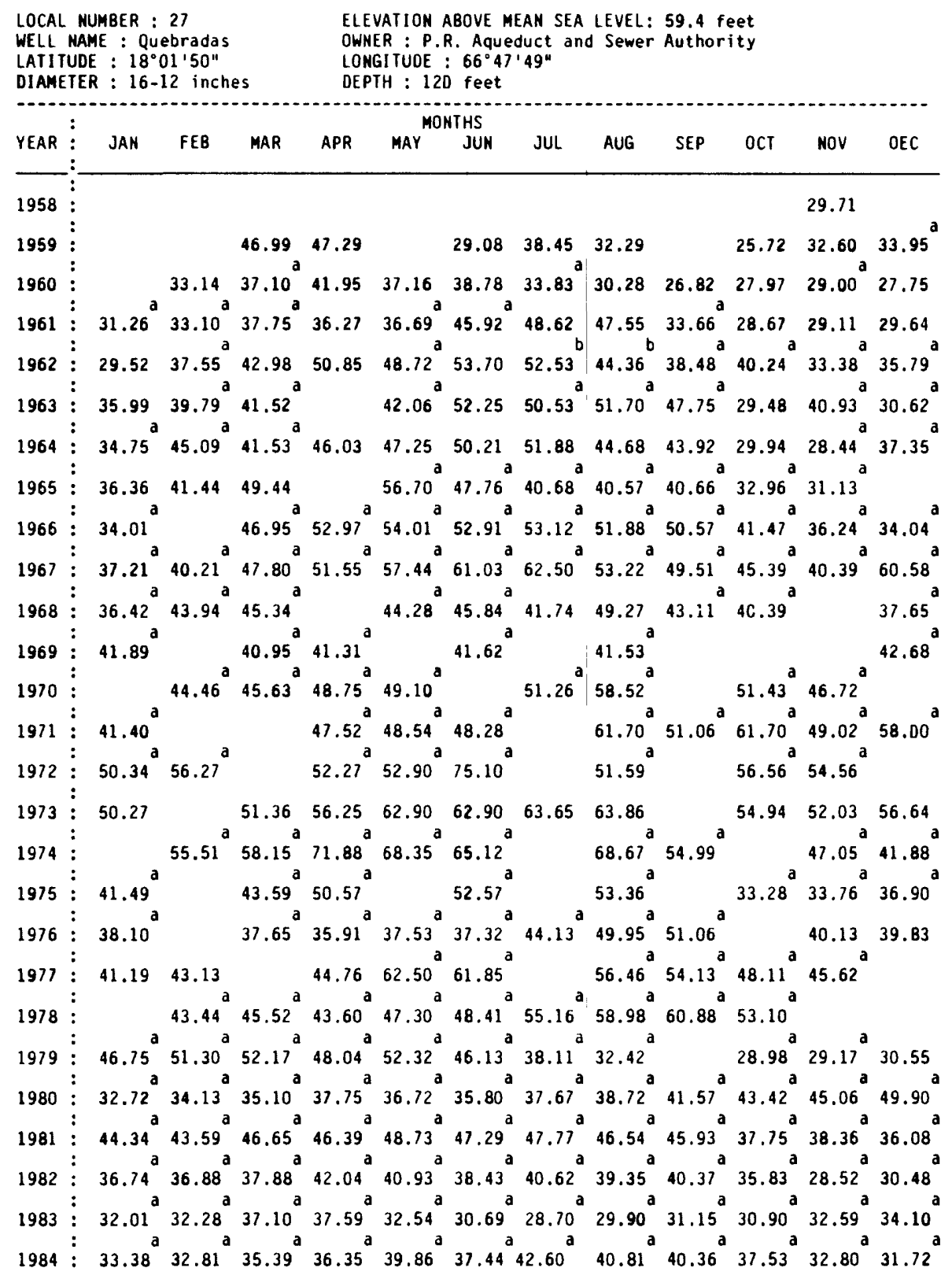

REMARKS: Well discont inued.

a pumping, b recently pumped, e estimated, $h$ tape measurement, $j$ lowest water level recorded,

n nearby pumping well 


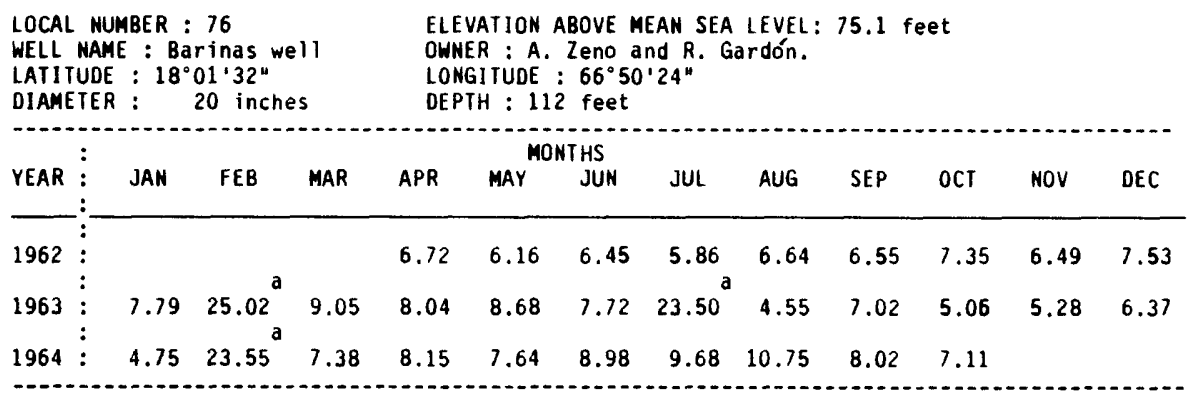

a pumping, b recently pumped, e estimated, $h$ tape measurement, $j$ lowest water level recorded, n nearby pumping well

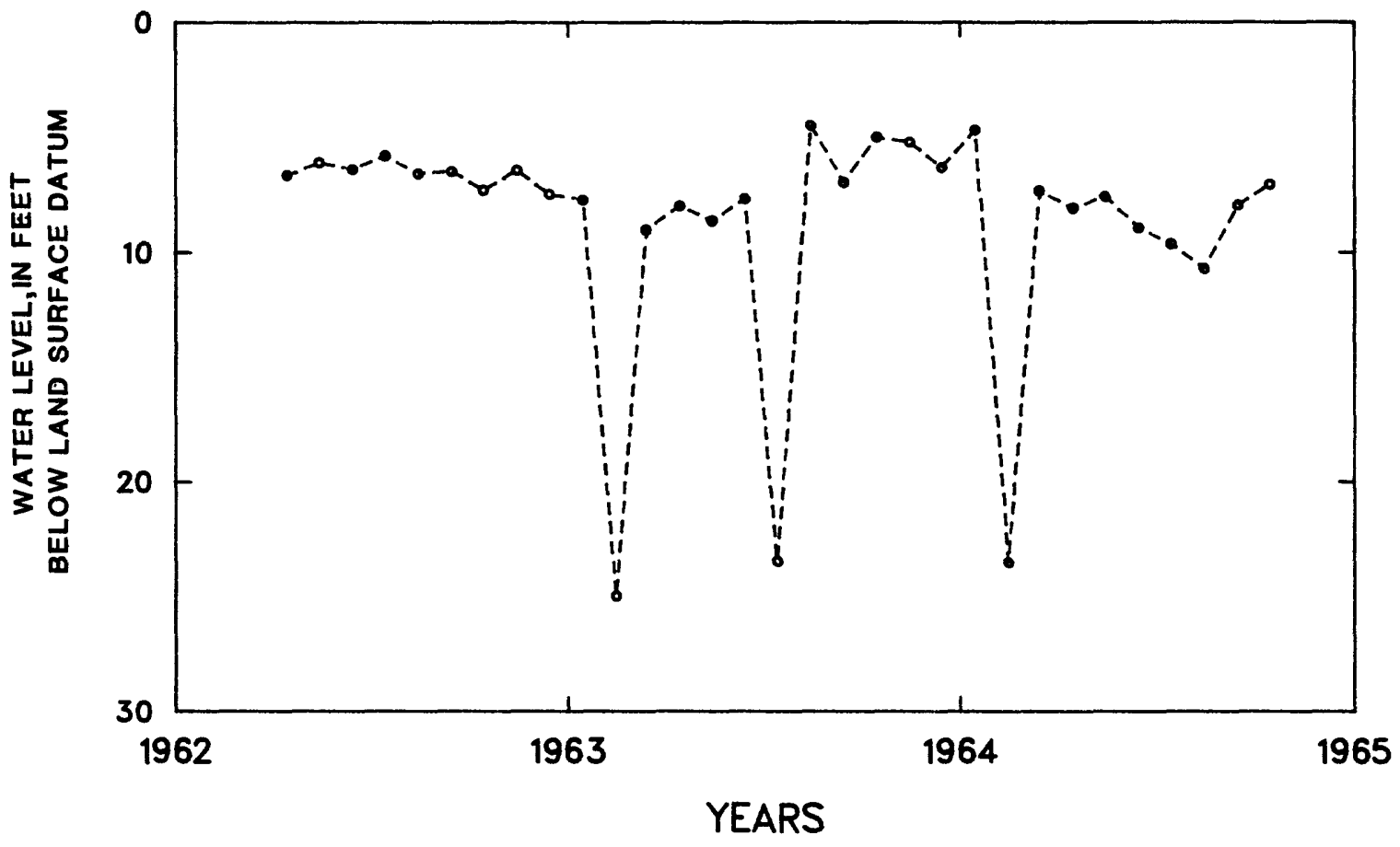

Figure 23.--Ground-water levels at Barinas, local number 76 . 


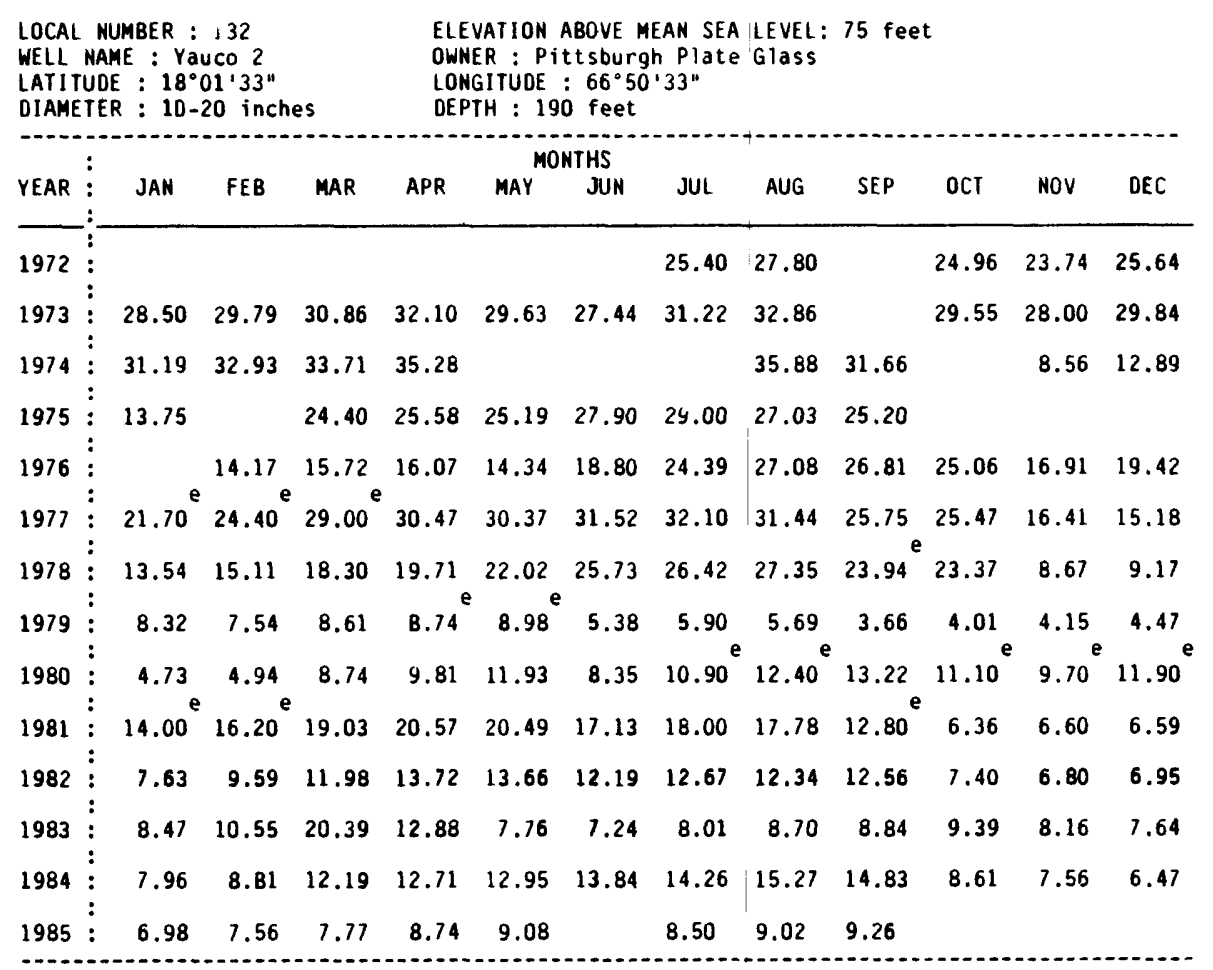

$\begin{array}{ll}\text { LOCAL NUMBER : } 134 & \text { ELEVATION ABOVE MEAN SEA LEVEL: } 87 \text { feet } \\ \text { WELL NAME : YauCO } 4 \text { or UCC } 1 \text { OWNER : Un ion Carbide Corporation } \\ \text { LATITUOE : } 18^{\circ} 01^{\prime} 20^{\circ} & \text { LONGITUOE : } 66^{\circ} 50^{\prime} 32^{\prime \prime} \\ \text { DIAMETER : } & \text { DEPTH : } 163^{\circ} \text { feet }\end{array}$

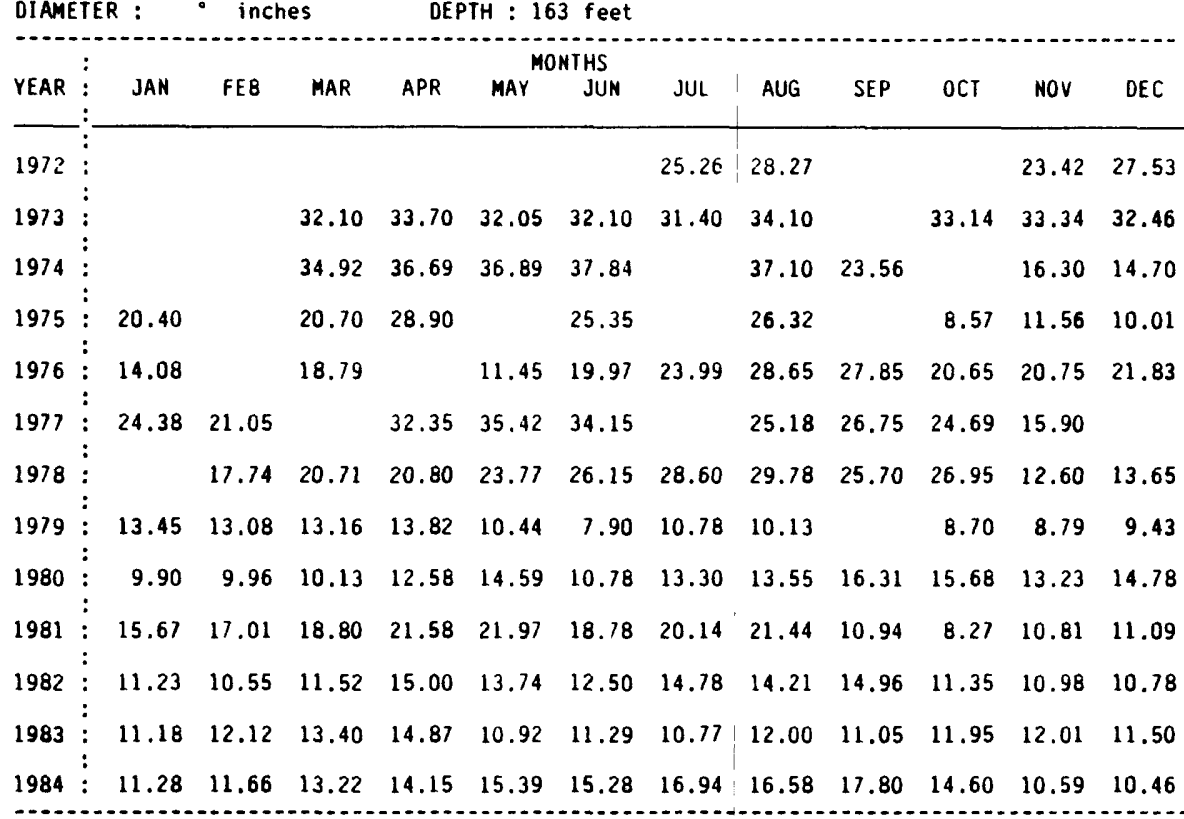

a pumping, b recently pumped, e estimated, $h$ tape measurement, j lowest water level recorded, n nearby pumping well 
THIS PAGE WAS LEFT BLANK INTENTIONALLY

97 


\begin{tabular}{|c|c|c|c|c|c|c|c|c|c|c|c|c|}
\hline $\begin{array}{l}\text { LOCAL } \\
\text { WELL } \\
\text { LATITI } \\
\text { DIAME }\end{array}$ & $\begin{array}{l}\text { NUMBER : } \\
\text { NAME : La } \\
\text { UDE : } 1 B^{\circ} \\
\text { TER : }\end{array}$ & $\begin{array}{l}77 \\
51 \text { Palom } \\
01.07 " \\
\text { inch }\end{array}$ & & $\begin{array}{l}\text { ELE } \\
\text { OWN } \\
\text { LON } \\
\text { DEP }\end{array}$ & $\begin{array}{l}\text { VATION } \\
\text { ER : Fé } \\
\text { GITUDE } \\
\text { TH: } 17\end{array}$ & $\begin{array}{l}\text { ABOVE ME } \\
\text { lix Gon } \\
66^{*} 52 \\
5 \text { feet }\end{array}$ & $\begin{array}{l}\text { EAN SEA } \\
\text { zález } \\
\text { '50" }\end{array}$ & LEVEL: & $105 \mathrm{fe}$ & & & \\
\hline$\cdots+-$ & : & 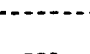 & & & MO & NTHS & & & & & & \\
\hline YEAR & JAN & FEB & MAR & APR & MAY & JUN & JUL & AUG & SEP & $\mathrm{OCT}$ & NOV & DEC \\
\hline 1962 & : & & & & & & & & & 12.02 & 11.56 & \\
\hline 1963 & : & 15.59 & & $18.67^{a}$ & 16.54 & 9.35 & 10.00 & & 8.52 & 7.55 & 7.47 & 8.43 \\
\hline 1964 & $: 12.11$ & 11.98 & 9.40 & 10.50 & 10.32 & 12.83 & 15.11 & & & & & \\
\hline 1965 & : & & & & & 13.59 & 11.93 & 11.15 & 11.29 & 11.92 & 10.36 & \\
\hline 1966 & $: 10.36$ & & 12.39 & 12.30 & 13.89 & 14.18 & & 17.65 & 17.13 & 18.12 & 10.15 & 9.44 \\
\hline 1967 & $: \quad 12.59$ & 14.90 & 15.93 & 16.66 & 15.20 & 17.96 & $19.75^{a}$ & $24.47^{a}$ & 18.90 & $17 . B 7$ & 15.05 & 13.61 \\
\hline 1968 & $: \quad 15.13$ & 17.53 & 17.89 & & 19.18 & $23.86^{a}$ & $20.72^{a}$ & 17.39 & 11.65 & 11.23 & & 8.55 \\
\hline 1969 & 8.40 & & & & & 14.43 & & & & & & \\
\hline
\end{tabular}

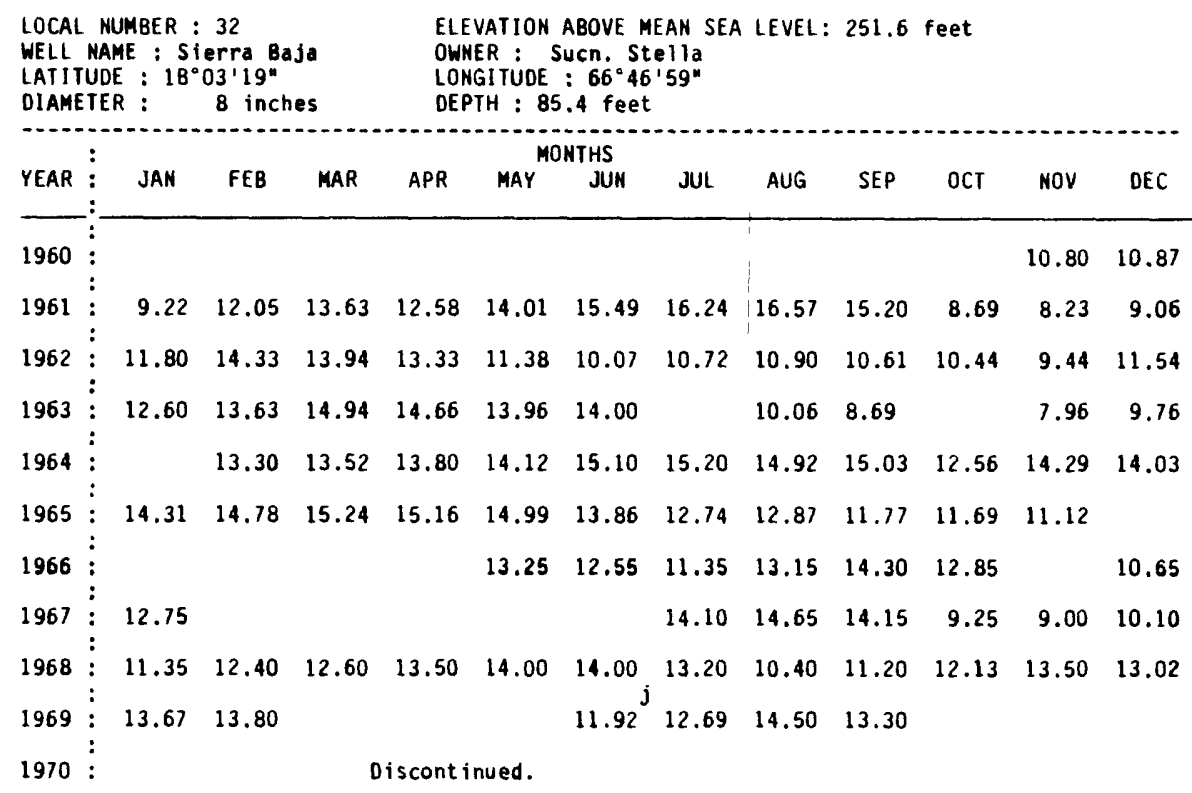

Remarks: Station discont inued, April 1970.

a pumping, b recently pumped, e estimated, $h$ tape measurement, j lowest water level recorded,

$n$ nearby pumping well 


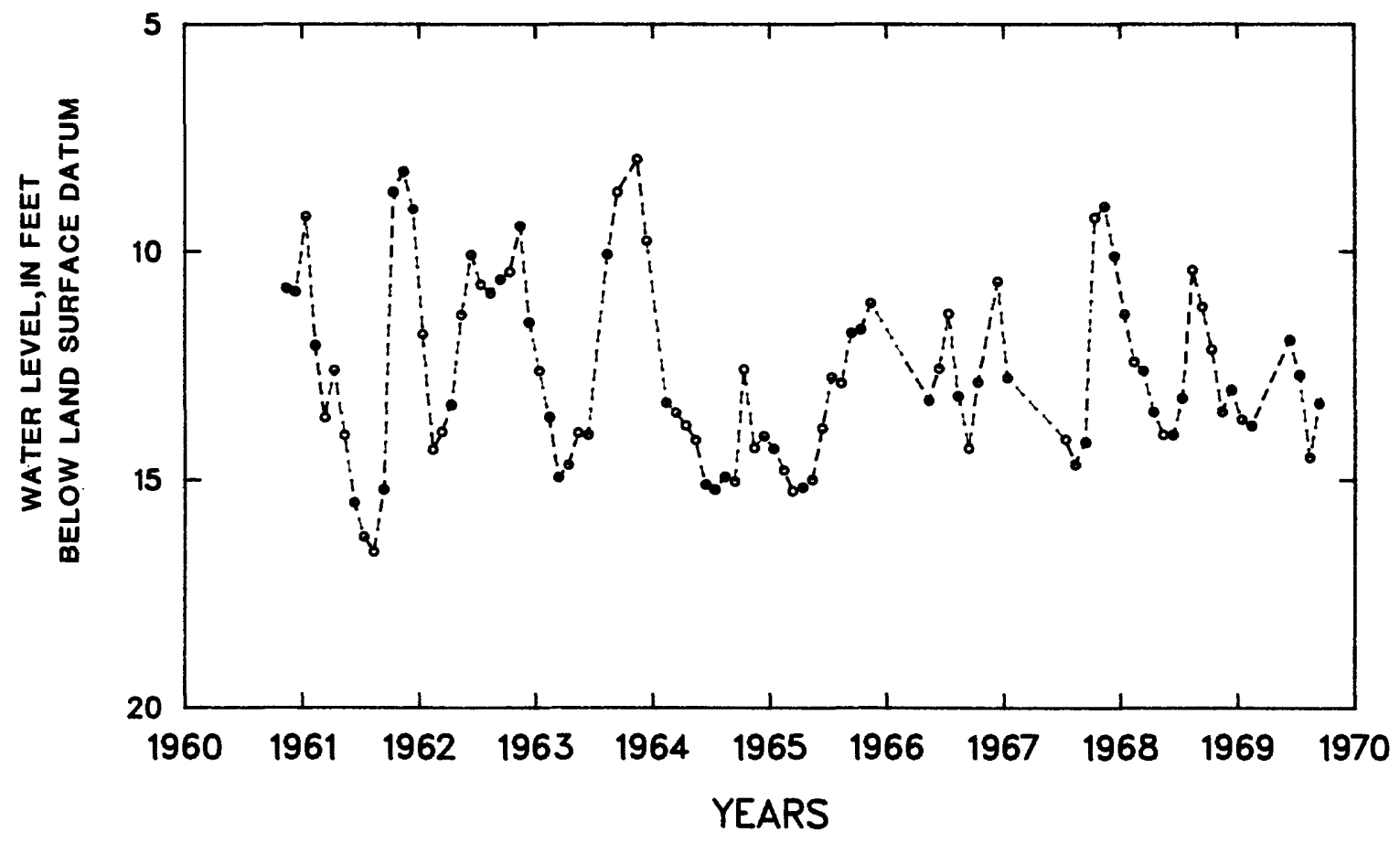

Figure 24.--Ground-water levels at Sierra Baja, local number 32. 


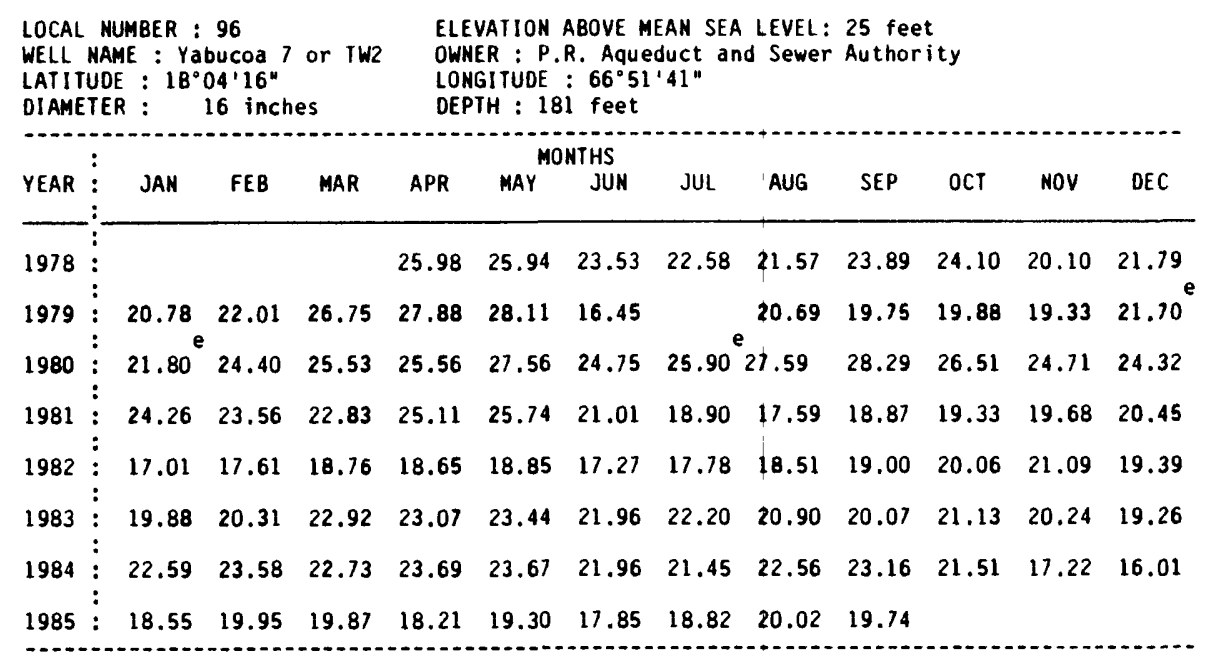

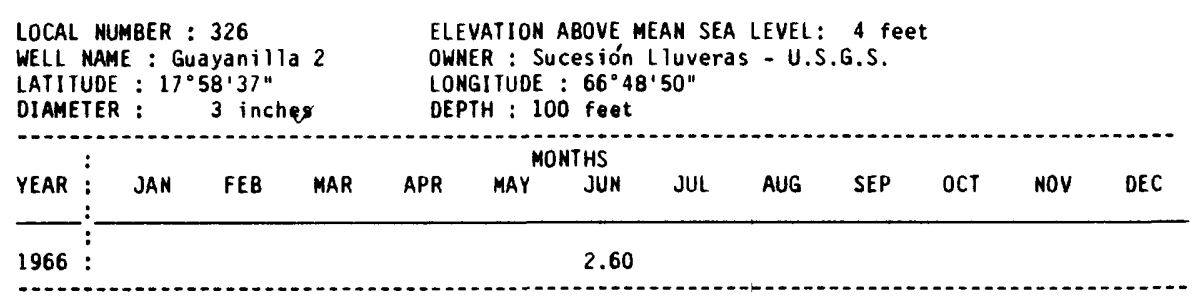

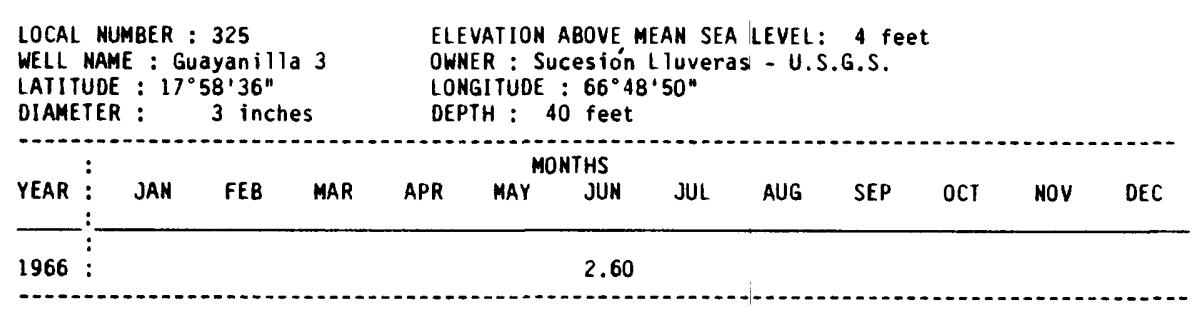

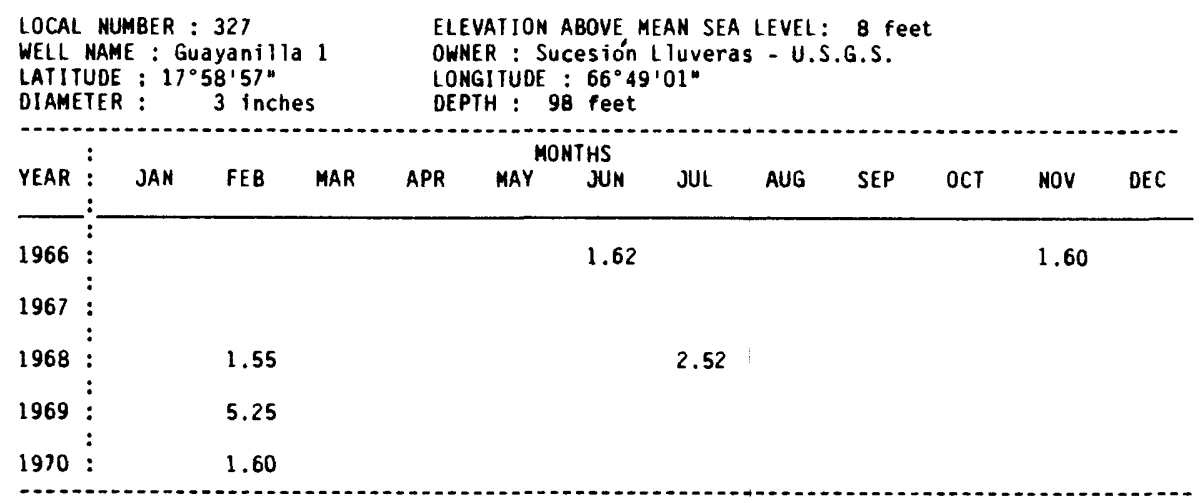

a puaping, b recently pumped, e estimated, $h$ tape measurement, $j$ lowest water level recorded, n nearby punping well 


\begin{tabular}{|c|c|c|c|c|c|c|c|c|c|c|c|c|}
\hline \multirow{2}{*}{$\begin{array}{l}\text { LOCAL } \\
\text { WELL } \\
\text { LATI TL } \\
\text { DIAME } \\
\text { YEAR }\end{array}$} & \multicolumn{3}{|c|}{$\begin{array}{l}\text { NUMBER : } 12 \\
\text { AME : Guánica } 1 \\
\text { IDE : } 17^{\circ} 58^{\prime} 25^{\prime \prime} \\
\text { ER : } 3 \text { inches }\end{array}$} & \multicolumn{7}{|c|}{$\begin{array}{l}\text { ELEVATION ABOVE MEAN SEA LEVEL: } 25 \text { feet } \\
\text { OWNER : P.R. Land AUthority } \\
\text { LONGITUDE: } 66^{\circ} 54^{\prime} 17^{\prime \prime} \\
\text { DEPTH : } 114 \text { feet }\end{array}$} & \multirow[b]{2}{*}{ Nov } & \multirow[b]{2}{*}{ DEC } \\
\hline & JAN & FEB & MAR & APR & MAY & $\begin{array}{l}\text { THS } \\
\text { JUN }\end{array}$ & JUL & AUG & SEP & OCT & & \\
\hline 1966 & & & & & & 2.55 & & & & & 4.00 & \\
\hline 1967 & & & & & & & & & 11.45 & & & \\
\hline 1968 & & 8.11 & & & & & & & & & & \\
\hline 1969 & & & & & & & & & & & & \\
\hline 1970 & & 5.86 & & & & & & & & & & \\
\hline
\end{tabular}

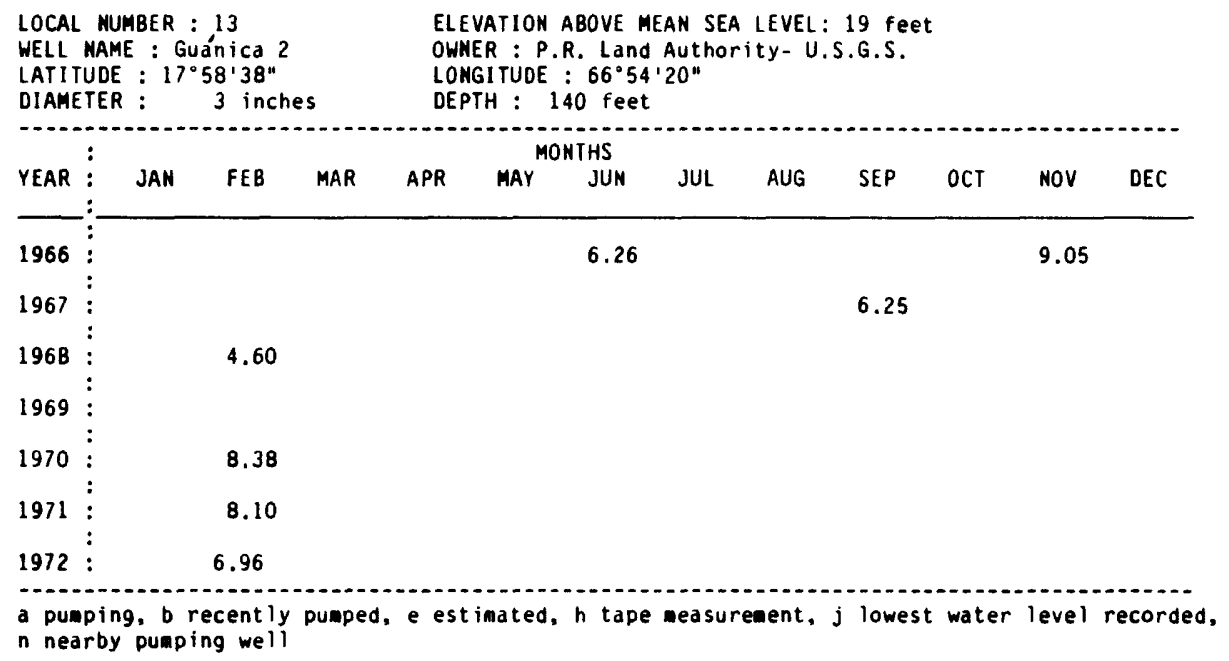




\begin{tabular}{|c|c|c|c|c|c|c|c|c|c|c|c|c|}
\hline $\begin{array}{l}\text { LOCAL } \\
\text { WELL } \\
\text { LATIT } \\
\text { DIAME }\end{array}$ & $\begin{array}{l}\text { MUMBER : } \\
\text { NAME : GU } \\
\text { UDE : } 17^{\circ} \\
\text { TER : } 13-\end{array}$ & $\begin{array}{l}328 \\
\text { ánica } 1 \\
58 \text { '58" } \\
16 \text { inch }\end{array}$ & & $\begin{array}{l}\text { ELE } \\
\text { OWM } \\
\text { LON } \\
\text { DEP }\end{array}$ & $\begin{array}{l}\text { VATION } \\
\text { IER : P } \\
\text { IGI TUDE } \\
\text { TH : } 8\end{array}$ & $\begin{array}{l}\text { ABOVE } \\
R \text {. Aque } \\
: 66^{\circ} 54 \\
5 \text { feet }\end{array}$ & $\begin{array}{l}\text { EEAN SE } \\
\text { duct a } \\
\text { ' } 12^{4}\end{array}$ & $\begin{array}{l}\text { LEVEL: } \\
\text { Sewer }\end{array}$ & $\begin{array}{r}9 \mathrm{f} \\
\text { Auth }\end{array}$ & & & \\
\hline & JAY & & & & & ONTHS & & & & & & \\
\hline YEAR & JAM & FEB & MAR & APR & MAY & JUN & JUL & AUG & SEP & OCT & NOV & DEC \\
\hline 1958 & $\vdots$ & & & & & & & $7.29^{b}$ & & & 8.03 & \\
\hline 1959 & : & & $13.03^{\mathrm{a}}$ & $14.42^{\mathrm{a}}$ & & $12.36^{a}$ & 10.04 & 11.83 & 9.90 & 13.06 & 9.53 & $11 . \mathrm{B} 6$ \\
\hline 1960 & $\vdots 13.18$ & 13.23 & 15.00 & 14.89 & 7.80 & 8.22 & 10.32 & 12.05 & 3.90 & 9.11 & 10.89 & 8.04 \\
\hline 1961 & 10.51 & 10.40 & 11.70 & 12.58 & 12.60 & 12.84 & $17.79^{\mathrm{a}}$ & 13.55 & $18.97^{\mathrm{a}}$ & 16.90 & 11.23 & \\
\hline 1962 & $\vdots 18.59^{\mathrm{a}}$ & 12.42 & 12.43 & 12.75 & 12.53 & $16.55^{\circ}$ & 11.78 & 9.66 & 8.97 & 10.08 & 11.69 & 11.96 \\
\hline 1963 & $\vdots 12.41$ & 13.27 & 15.25 & & 15.13 & 20.60 & 14.35 & & & 6.32 & 13.83 & 14.02 \\
\hline 1964 & 9.89 & 11.46 & $17.72^{\mathrm{a}}$ & 11.19 & 11.81 & 12.25 & 13.50 & 14.48 & 14.18 & 10.77 & 12.08 & 13.73 \\
\hline 1965 & $: 14.18$ & 13.96 & 16.98 & & 17.14 & $24.60^{\mathrm{a}}$ & 14.37 & $22.13^{\mathrm{a}}$ & $17.72^{\mathrm{a}}$ & $18.8 B$ & 17.57 & \\
\hline 1966 & ${ }^{:} 15.81^{\mathrm{a}}$ & & 11.45 & $18.18^{\circ}$ & $23.85^{\circ}$ & $24.69^{a}$ & & $18.09^{a}$ & 13.57 & 13.28 & 13.59 & 11.07 \\
\hline 1967 & $: 10.32$ & 11.72 & 11.66 & 13.14 & 14.00 & $19.95^{\mathrm{a}}$ & $20.91^{\mathrm{a}}$ & $20.77^{\mathrm{a}}$ & 14.57 & 14.81 & 14.50 & 11.80 \\
\hline 1968 & $: 12.00$ & $17.15^{\mathrm{a}}$ & $16.50^{\mathrm{a}}$ & $18.35^{\mathrm{a}}$ & $17.92^{\circ}$ & 15.66 & $19.73^{\circ}$ & $18.87^{\mathrm{a}}$ & $17.11^{\mathrm{a}}$ & 16.53 & & $16.82^{\mathrm{a}}$ \\
\hline 1969 & $: \quad 12.70$ & & $18.48^{a}$ & $21.35^{\mathrm{a}}$ & & $22.14^{\mathrm{a}}$ & & $25.68^{a}$ & & 23.00 & & $19.39^{\mathrm{a}}$ \\
\hline 1970 & $\vdots 20.80^{\mathrm{a}}$ & & $21.29^{\mathrm{a}}$ & $21.35^{\mathrm{a}}$ & $20.23^{\circ}$ & & $21.52^{\circ}$ & $21.37^{\mathrm{a}}$ & & 17.68 & $16.18^{a}$ & \\
\hline 1971 & $: \quad 17.66^{\mathrm{a}}$ & & $21.30^{a}$ & $20.20^{a}$ & $21.53^{\circ}$ & $19.22^{a}$ & $21.59^{\circ}$ & $23.06^{\mathrm{a}}$ & 17.69 & 19.65 & $22.14^{a}$ & $18.37^{\mathrm{a}}$ \\
\hline 1972 & $: 19.81^{\mathrm{a}}$ & $19.79^{\circ}$ & & $20.82^{a}$ & $17.65^{\circ}$ & $19.27^{\mathrm{a}}$ & & $20.30^{\mathrm{a}}$ & & 22.69 & $19.70^{\mathrm{a}}$ & \\
\hline 1973 & : 20.44 & & 21.31 & 24.22 & 22.60 & 22.62 & 21.42 & 21.08 & & 29.10 & 18.28 & 30.10 \\
\hline 1974 & : & 12.06 & 20.64 & 23.11 & 24.80 & 22.98 & & 20.96 & 14.75 & & 10.66 & 10.93 \\
\hline 1975 & 15.80 & & 23.50 & 34.82 & & 36.90 & & & & & 19.87 & 21.00 \\
\hline 1976 & 29.25 & & 39.35 & & 10.77 & 22.13 & $24.21^{\circ}$ & & & 24.35 & $14.18^{\circ}$ & 25.80 \\
\hline 377 & 28.28 & & & & & & & & & & & \\
\hline
\end{tabular}

REMARKS: Well discont inued, March 1977.

\begin{tabular}{|c|c|c|c|c|c|c|c|c|c|c|c|c|}
\hline $\begin{array}{l}\text { LOCAL } \\
\text { WELL } \\
\text { LATI } \\
\text { DIAME }\end{array}$ & $\begin{array}{l}\text { UMBER } \\
\text { ME : } \\
\text { E : } 1 \\
R:\end{array}$ & $\begin{array}{l}330 \\
\text { ánica } \\
58 \cdot 15 " \\
3 \text { inc }\end{array}$ & & & $\begin{array}{l}\text { AT IOS } \\
R: \\
\text { II IUDE } \\
H:\end{array}$ & $\begin{array}{l}\text { ABOVE } \\
\text { R. Aque } \\
66^{\circ} 5 \\
5 \text { feet }\end{array}$ & $\begin{array}{l}\text { AN S } \\
\text { uCt } \\
38^{\prime \prime}\end{array}$ & $\begin{array}{l}\text { LEVEL: } \\
\text { o Sewer }\end{array}$ & $\begin{array}{r}5 \mathrm{fe} \\
\text { Autho }\end{array}$ & ty & S.G.S & \\
\hline YEAR & JAN & FEB & MAR & APR & MAY" & $\begin{array}{l}\text { NTHS } \\
\text { JUN }\end{array}$ & JUL & AUG & SEP & DCT & NOV & DEC \\
\hline 1966 & & & & & & 0.35 & & & & & 0.00 & \\
\hline 1967 & & & & & & & & & 2.25 & & & \\
\hline 1968 & & 1.27 & & & & & & & & & & \\
\hline 1969 & & & & & & & & & & & & \\
\hline 1970 & & -1.07 & & & & & & & & & & \\
\hline 1971 & & -0.96 & & & & & & & & & & \\
\hline
\end{tabular}

a pumping, b recently pumped, e est imated, $n$ tape measurement, $j$ lowest water level recorded, n nearby pumping well 


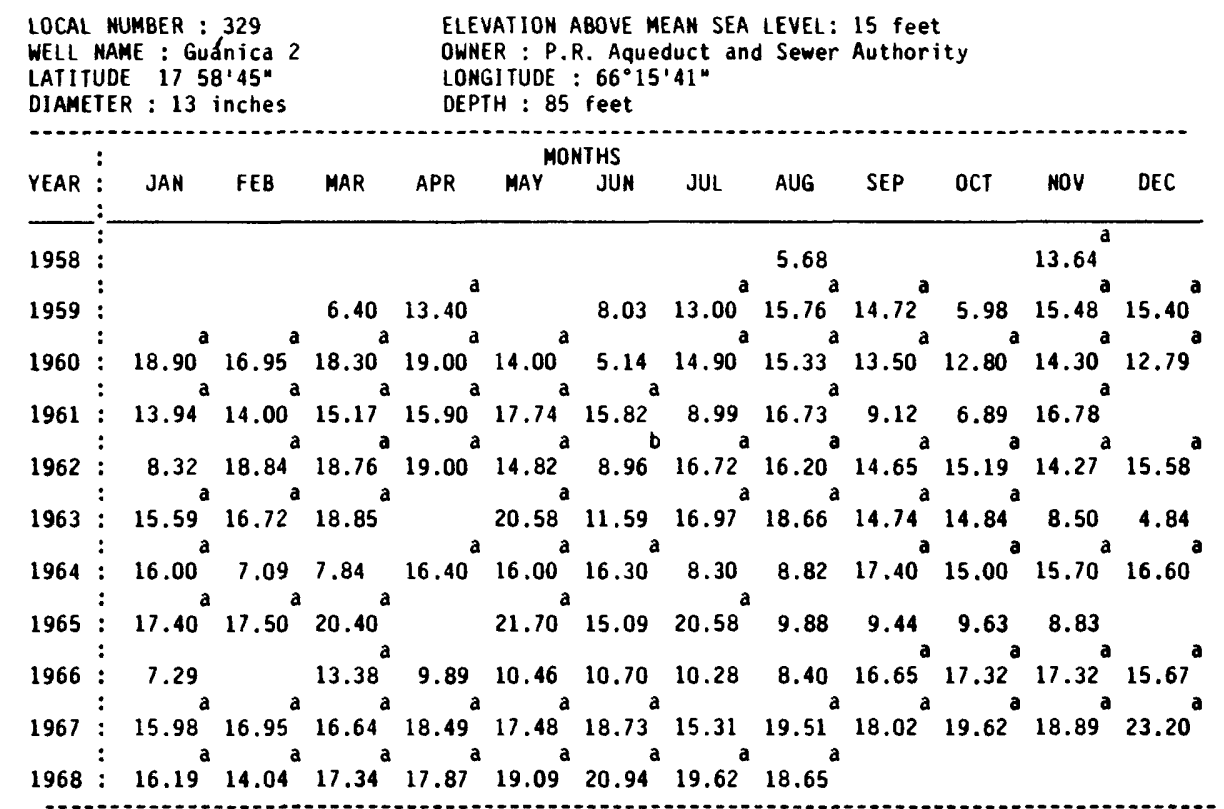

REMARKS: Well discontinued, October 1968.

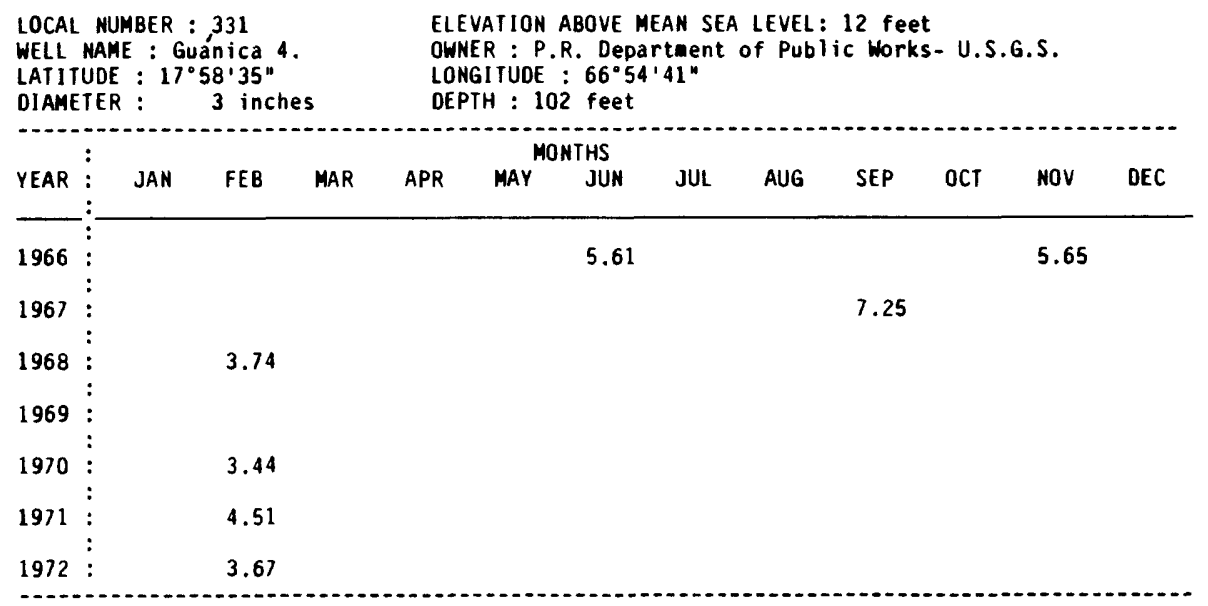

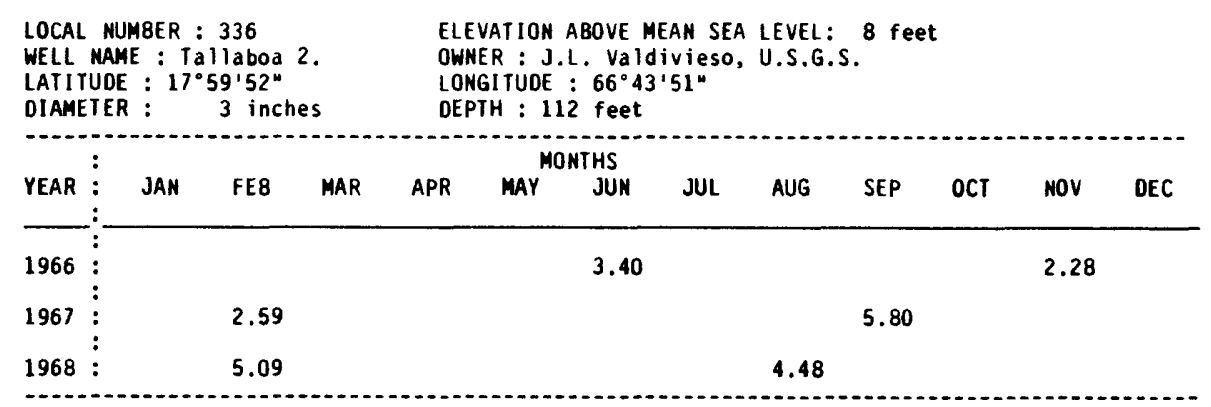

a puaping, b recently pumped, e estiaated, $h$ tape measurement, j lowest water level recorded, n nearby pumping well 


\begin{tabular}{|c|c|c|c|c|c|c|c|c|c|c|c|c|}
\hline $\begin{array}{l}\text { LOCAL } \\
\text { WELL } \\
\text { LATII } \\
\text { DIAME }\end{array}$ & $\begin{array}{l}\text { NUMBER } \\
\text { MAME : } \\
\text { IUDE : } 17 \\
\text { ETER : }\end{array}$ & $\begin{array}{l}337 \\
\text { yanil } \\
9 \cdot 22 \text { " } \\
3 \text { inc }\end{array}$ & & & $\begin{array}{l}\text { ATION } \\
\text { R : S } \\
\text { I TUDE } \\
H: 1\end{array}$ & $\begin{array}{l}\text { BOVE } \\
\text { cesión } \\
66^{\circ} 48 \\
\text { feet }\end{array}$ & $\begin{array}{l}\text { AN SE } \\
\text { luver } \\
15^{4}\end{array}$ & $\begin{array}{l}\text { LEVEL } \\
-U .\end{array}$ & $\begin{array}{l}4 \mathrm{fe} \\
\text { a.s. }\end{array}$ & & & \\
\hline YEAR & $:$ & FEB & MAR & APR & MAY & $\begin{array}{l}\text { ITHS } \\
\text { JUN }\end{array}$ & JUL & AUG & SEP & OCI & NOV & DEC \\
\hline 1966 & : & & & & & 1. BO & & & & & 1.15 & \\
\hline 1967 & : & & & & & & & & & & & \\
\hline 1968 & $:$ & 1.00 & & & & & 3.40 & & & & & \\
\hline 1969 & : & 2.55 & & & & & & & & & & \\
\hline
\end{tabular}

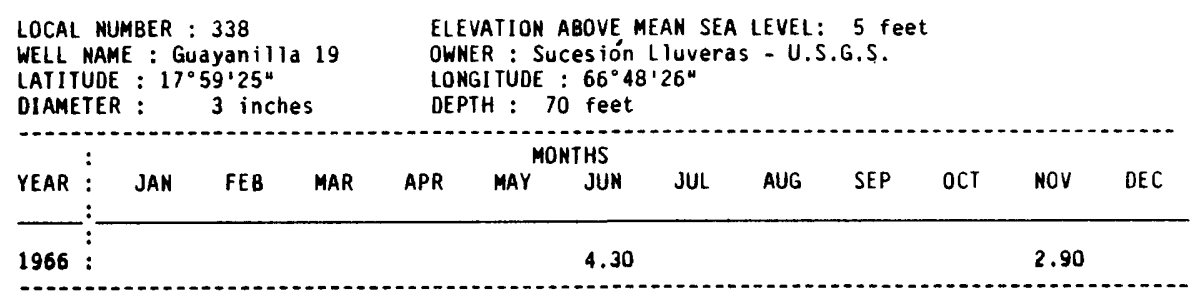

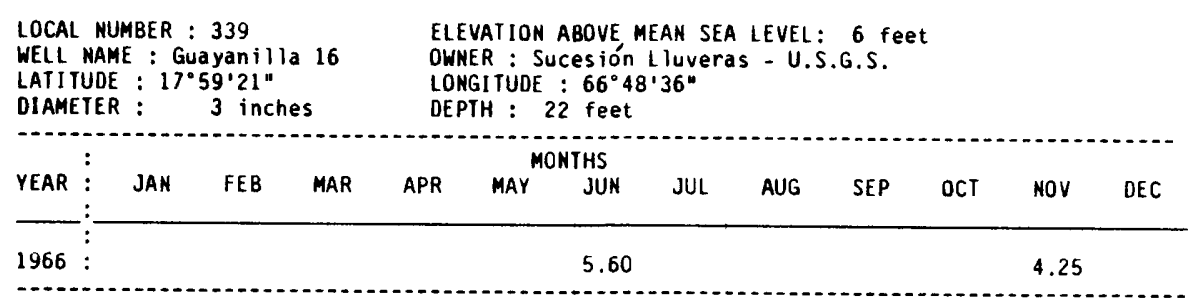

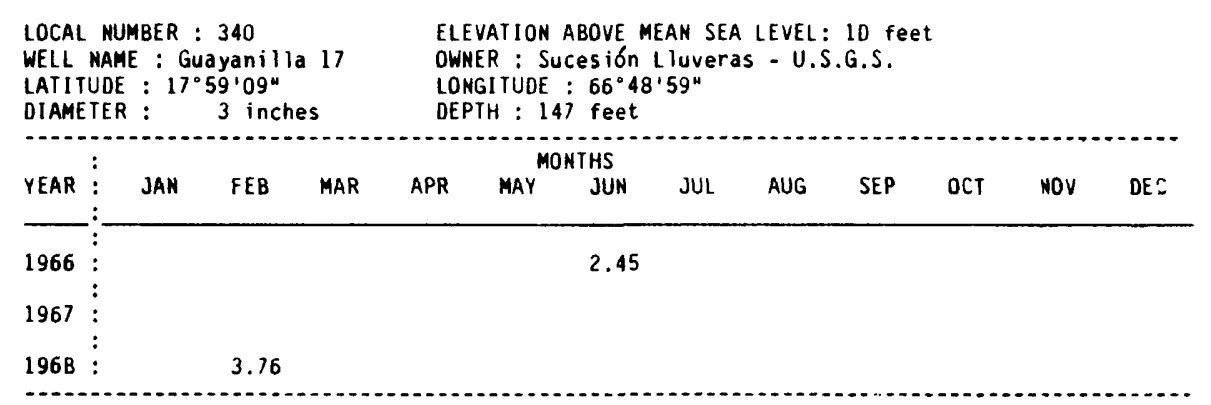

a pumping, b recently pumped, e estimated, h tape measurement, : : owost water leve' recorded. n nearby pumping well 


\begin{tabular}{|c|c|c|c|c|c|c|c|c|c|c|c|c|c|}
\hline $\begin{array}{l}\text { LOCAL } \\
\text { WELL } \\
\text { LATIT } \\
\text { DIAME }\end{array}$ & $\begin{array}{l}\text { NL } \\
\text { NAI } \\
\text { TUD } \\
\text { TE }\end{array}$ & $\begin{array}{l}\text { UMBER : } \\
\text { ME : Cn } \\
\text { E : } 17^{\circ} \\
R: 20\end{array}$ & $\begin{array}{l}16 \\
\text { t.S. Fr } \\
59^{\prime} 22^{\prime \prime} \\
\text { inches }\end{array}$ & ancisco & $\begin{array}{l}\text { ELE } \\
\text { OWN } \\
\text { LON } \\
\text { DEP }\end{array}$ & $\begin{array}{l}\text { VAIION } \\
\text { ER : CeI } \\
\text { GITUDE } \\
\text { TH : } 1 \mathrm{~B}\end{array}$ & $\begin{array}{l}\text { ABOVE M } \\
\text { ntral L } \\
: 66^{\circ} 49 \\
5 \text { feet }\end{array}$ & $\begin{array}{l}\text { EAN SEA } \\
\text { luveras } \\
\text { ' } 5 B^{\prime \prime}\end{array}$ & $\begin{array}{l}\text { LEVEL } \\
\text { (San }\end{array}$ & $\begin{array}{l}29.6 \mathrm{ft} \\
\text { ancisco }\end{array}$ & $\begin{array}{l}\text { eet } \\
\text { ) }\end{array}$ & & \\
\hline & : & & & & & MO & NTHS & & & & & & \\
\hline YEAR & : & JAN & FEB & MAR & APR & MAY & JUN & JUL & AUG & SEP & OCT & HOV & DEC \\
\hline 1960 & : & & & & & & & & & & & 6.12 & 5.72 \\
\hline 1961 & : & 5.51 & 5.70 & 6.82 & 8.06 & 7.62 & 7.69 & 7.98 & B. 91 & 6.37 & 6.06 & 6.04 & 6.81 \\
\hline 1962 & : & 8.24 & 9.23 & 10.30 & 10.80 & 7.03 & 9.09 & 9.44 & 8.75 & 10.12 & 10.39 & 10.13 & 13.22 \\
\hline 1963 & : & 15.70 & 16.03 & 13.61 & 14.73 & 14.82 & 14.15 & 16.73 & 6.46 & 5.51 & 5.89 & 4.73 & 9.72 \\
\hline 1964 & : & 14.30 & 14.75 & 15.35 & 15.97 & 15.01 & 12.39 & 14.78 & 11.98 & 12.07 & 9.54 & 12.55 & 11.47 \\
\hline 1965 & : & 15.64 & 15.27 & 15.82 & 14.99 & $12.8 \mathrm{~B}$ & 9.08 & 4.73 & 8.41 & B. 36 & 4.84 & 4.39 & \\
\hline 1966 & $\vdots$ & 9.06 & & 10.81 & 11.13 & 11.70 & 9.13 & 9.42 & 9.29 & $1 B .54$ & 14.11 & B. 15 & 5.94 \\
\hline 1967 & $\vdots$ & 12.76 & 15.82 & 17.30 & 13.53 & 13.54 & 18.80 & 21.10 & 17.87 & & & & \\
\hline 1968 & : & 15.03 & 17.95 & 15.40 & 18.87 & 17.40 & 15.79 & 21.10 & 13.75 & 13.13 & 11.84 & 8.00 & 5.62 \\
\hline 1969 & : & 5.40 & 9.08 & 6.91 & 8.82 & 19.92 & 18.56 & & & & & & \\
\hline 1970 & $\vdots$ & & & 8.82 & 19.25 & 18.56 & & & & & & & \\
\hline 1971 & $\vdots$ & & & 9.54 & 9.59 & 11.50 & 7.12 & 10.72 & 13.70 & 6.41 & 6.70 & $6.8 \mathrm{~B}$ & \\
\hline 1972 & : & 8.91 & 14.39 & & 10.90 & 11.53 & 15.60 & & 18.43 & & 20.14 & 7.37 & 18.67 \\
\hline 1973 & : & & & 19.64 & 26.20 & 26.88 & 27.79 & 26.77 & 27.21 & & 19.29 & 16.80 & 19.29 \\
\hline 1974 & : & & 22.19 & 19.54 & 23.14 & 23.09 & 23.38 & & & & & 20.95 & \\
\hline 1975 & : & 29.90 & & 35.76 & & & 22.05 & & 22.81 & & 6.14 & & \\
\hline 1976 & : & & & 8.43 & & 8.90 & 10.28 & & & & & 7.89 & 11.37 \\
\hline 1977 & : & 20.15 & 19.39 & & 19.98 & & 26.27 & & 19.56 & 21.26 & 17.93 & 12.49 & \\
\hline 1978 & : & 16.36 & & 12.54 & 12.15 & 11.96 & 15.76 & 18.74 & 22.62 & 18.40 & 15.73 & 9.02 & B. 49 \\
\hline 1979 & : & 8.27 & 12.10 & 16.57 & 13.88 & 14.86 & 5.28 & 7.58 & 7.42 & & 5.33 & 4.43 & 5.00 \\
\hline 1980 & : & 5.92 & 7.45 & 11.50 & 11.08 & 9.14 & & 10.38 & 12.31 & 12.10 & 11.06 & 11.06 & 13.03 \\
\hline 1981 & : & 13.09 & 10.00 & 14.35 & 10.19 & 11.67 & 7.10 & 13.41 & & 5.67 & 4.60 & 6.45 & 4.90 \\
\hline 1982 & : & 8.57 & 9.53 & 6.75 & 8.01 & 5.88 & 5.84 & 6.09 & 6.98 & 3.47 & 5.14 & 5.28 & 4.26 \\
\hline 1983 & $\vdots$ & 5.14 & 5.57 & 5.37 & 5.58 & 4.43 & & 5.64 & 6.57 & 6.56 & 6.23 & 6.15 & 6.30 \\
\hline 1984 & : & 5.98 & 4.94 & 6.44 & 6.62 & 6.75 & 5.91 & 1.85 & 7.34 & 8.28 & 0.35 & 4.49 & 4.68 \\
\hline 1985 & : & 6.80 & 6.18 & 7.3 & & & & & & & & & \\
\hline
\end{tabular}

a pumping, b recently pumped, e estimated, $h$ tape measurement, $j$ lowest water level recorded, n nearby puping well 


\begin{tabular}{|c|c|c|c|c|c|c|c|c|c|c|c|c|}
\hline $\begin{array}{l}\text { LOCAL } \\
\text { WELL } \\
\text { LATIT } \\
\text { DIAME }\end{array}$ & $\begin{array}{l}\text { JMBER } \\
\text { AE : } \\
: 17 \\
:\end{array}$ & $\begin{array}{l}341 \\
\text { yani } \\
9 ' 10^{\prime} \\
3 \text { inc }\end{array}$ & 18. & & $\begin{array}{l}\text { ATIOI } \\
\text { R : } \\
\text { ITUD } \\
\text { H : }\end{array}$ & $\begin{array}{l}\text { BOVE, } \\
\text { esión } \\
66^{\circ} 4 \\
\text { feet }\end{array}$ & $\begin{array}{l}\text { AN SE } \\
\text { luver } \\
\text { 12" }\end{array}$ & $\begin{array}{l}\text { LEVEL } \\
- \text { U.S }\end{array}$ & $\begin{array}{l}14 \mathrm{fe} \\
. \mathrm{S} .\end{array}$ & & & \\
\hline & & & & & & THS & & & & & & \\
\hline YEAR & JAN & FEB & MAR & APR & MAY & JUN & JUL & AUG & SEP & OCT & NOV & DEC \\
\hline 1966 & & & & & & 1.20 & & & & & 1.60 & \\
\hline
\end{tabular}

a pumping, b recently pumped, e estimated, $h$ tape measurement, $j$ lowest water level recorded, n nearby pumping wel1 


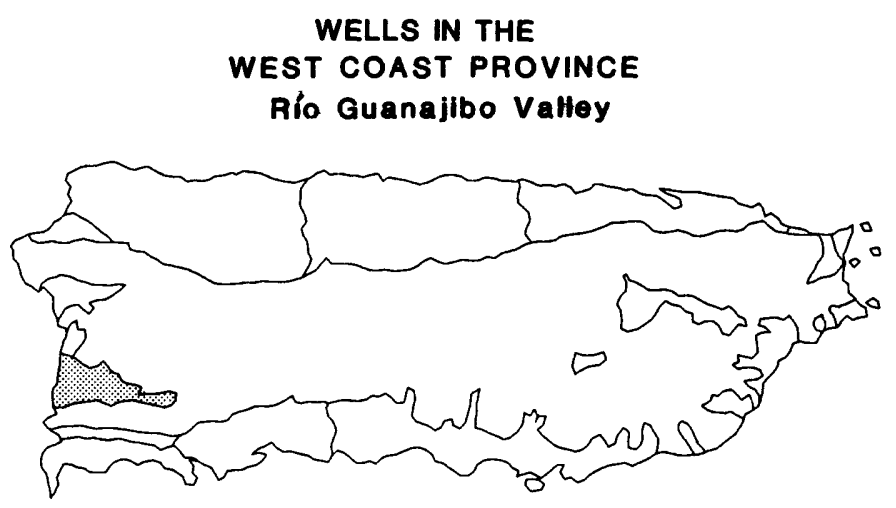

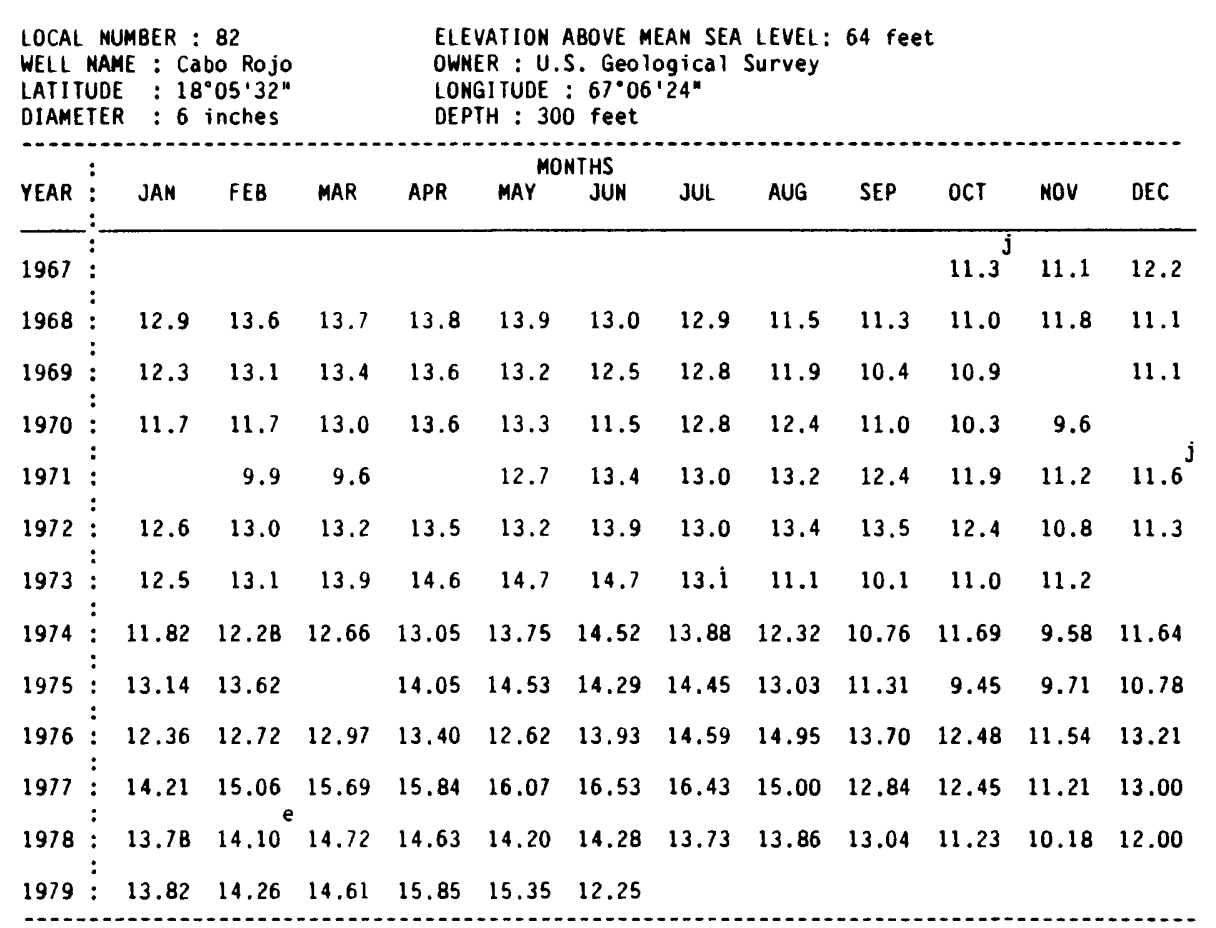

a pumping, b recently pumped, e estimated, $h$ tape measurement, $j$ lowest water level recorded, n nearby pumping well 


\begin{tabular}{|c|c|c|c|c|c|c|c|c|c|c|c|c|c|}
\hline $\begin{array}{l}\text { LOCAL } \\
\text { WELL } \\
\text { LAT IT } \\
\text { DIAME }\end{array}$ & & $\begin{array}{l}\text { BER : } \\
: \quad \text { Ro } \\
: 18^{\circ} \mathrm{C} \\
: 12-1\end{array}$ & $\begin{array}{l}r 10 \\
34 " \\
\text { inche }\end{array}$ & & $\begin{array}{l}\text { ELEV } \\
\text { OWNE } \\
\text { LONG } \\
\text { DEPT }\end{array}$ & $\begin{array}{l}\text { ATION } \\
R: \text { P. } \\
\text { ITUDE } \\
H: 105\end{array}$ & $\begin{array}{l}\text { BOVE ME } \\
\text { Aqued } \\
66^{\circ} 05 \\
\text { feet }\end{array}$ & $\begin{array}{l}S \\
t \\
\text { " }\end{array}$ & i & 1 & & & \\
\hline YEAR & : & JAN & FEB & MAR & APR & MAY & $\begin{array}{l}\text { THS } \\
\text { JUN }\end{array}$ & JUL & AUG & SEP & $\mathrm{OCT}$ & NOV & DEC \\
\hline 1960 & : & & & & & & & 11.31 & 12.25 & 11.44 & 11.84 & 11.01 & 11.99 \\
\hline 1961 & : & 12.10 & 12.24 & 12.58 & 12.89 & 12.14 & 12.39 & 12.13 & 12.10 & 12.12 & 12.16 & 11.84 & \\
\hline 1962 & $\vdots$ & 13.63 & 12.57 & $13.43^{\mathrm{a}}$ & 13.74 & 13.22 & 12.40 & 12.53 & 12.43 & 12.53 & 12.43 & 12.42 & 2.59 \\
\hline 1963 & : & 12.76 & 13.51 & 12.77 & $13.49^{a}$ & 12.31 & 12.25 & $12.24^{\mathrm{a}}$ & 11.50 & 11.67 & 11.59 & $13.21^{\mathrm{a}}$ & $13.10^{\circ}$ \\
\hline 1964 & $\vdots$ & 12.73 & $13.56^{\mathrm{a}}$ & $13.57^{\circ}$ & 12.87 & 13.53 & 13.55 & 13.05 & 12.62 & 12.58 & 12.84 & 13.22 & 13.28 \\
\hline 1965 & $\vdots$ & 12.75 & 13.65 & $13.68^{d}$ & & $12.71^{d}$ & $12.59^{\circ}$ & $13.73^{\mathrm{d}}$ & $12.24^{\alpha}$ & $12.77^{d}$ & 11.44 & 10.76 & 12.54 \\
\hline 1966 & : & 12.85 & $13.80^{\mathrm{a}}$ & $13.87^{\mathrm{a}}$ & $13.29^{\mathrm{a}}$ & 12.80 & $13.00^{\mathrm{a}}$ & $12.90^{\mathrm{a}}$ & $12.93^{\mathrm{a}}$ & $13.50^{\mathrm{a}}$ & $13.70^{\mathrm{a}}$ & $12.97^{\mathrm{a}}$ & $12.80^{\mathrm{a}}$ \\
\hline 1967 & $\vdots$ & $12.73^{\mathrm{a}}$ & $12.85^{\mathrm{a}}$ & $13.81^{\mathrm{a}}$ & & $13.04^{\circ}$ & $13.93^{\mathrm{a}}$ & $13.55^{\mathrm{a}}$ & $13.33^{\mathrm{a}}$ & $13.20^{\mathrm{a}}$ & $13.14^{\mathrm{a}}$ & $13.36^{\mathrm{a}}$ & $14.34^{\mathrm{a}}$ \\
\hline 1968 & : & $15.61^{a}$ & $14.82^{\mathrm{a}}$ & $15.05^{a}$ & $14.13^{\mathrm{a}}$ & $14.58^{a}$ & $15.14^{\mathrm{a}}$ & 11.42 & 12.55 & $13.05^{\mathrm{a}}$ & $14.50^{\mathrm{a}}$ & & $15.30^{\mathrm{a}}$ \\
\hline 1969 & $\vdots$ & 14.25 & & $15.13^{a}$ & $14.61^{a}$ & & $15.62^{a}$ & & 15.05 & & & & 13.86 \\
\hline 1970 & : & $15.27^{a}$ & & $16.15^{a}$ & $15.35^{\mathrm{a}}$ & $15.45^{\mathrm{a}}$ & & $15.29^{\mathrm{a}}$ & $14.78^{\mathrm{a}}$ & 12.91 & & & \\
\hline 1971 & : & & & $15.97^{\mathrm{a}}$ & & $15.28^{a}$ & & $12.83^{a}$ & $14.62^{\mathrm{a}}$ & $15.09^{\mathrm{a}}$ & $15.55^{a}$ & $14.91^{\mathrm{a}}$ & \\
\hline 1972 & $\vdots$ & & & & & & & & & & & & \\
\hline 973 & $\vdots$ & & & & & 37.30 & & & & & & & \\
\hline 1974 & $\vdots$ & & & $23.62^{\mathrm{a}}$ & $20.98^{\mathrm{a}}$ & $23.92^{\mathrm{a}}$ & $23.56^{\mathrm{a}}$ & & $21.52^{\mathrm{a}}$ & $14.75^{a}$ & & $20.25^{a}$ & $20.86^{a}$ \\
\hline 1975 & : & $20.73^{a}$ & & $19.44^{a}$ & $21.48^{\mathrm{a}}$ & & $17.08^{a}$ & & $19.90^{\mathrm{a}}$ & & $17.53^{\mathrm{a}}$ & & $16.05^{\mathrm{d}}$ \\
\hline 1976 & : & 10.31 & & $14.56^{a}$ & & 11.69 & $16.53^{a}$ & $15.97^{\mathrm{a}}$ & 11.84 & 12.66 & $13.49^{\mathrm{a}}$ & $13.62^{\mathrm{a}}$ & $14.40^{\mathrm{a}}$ \\
\hline 1977 & : & $14.23^{\circ}$ & 14.25 & & 10.71 & 11.17 & $16.85^{a}$ & 10.40 & 11.50 & & $12.06^{a}$ & & \\
\hline 1978 & : & & $15.84^{\mathrm{a}}$ & $16.65^{a}$ & $16.10^{\mathrm{a}}$ & $14.18^{a}$ & 11.40 & 12.73 & 13.78 & $13.72^{\mathrm{a}}$ & $11.71^{\mathrm{a}}$ & $12.99^{\mathrm{a}}$ & $13.26^{\mathrm{a}}$ \\
\hline 1979 & : & 13.30 & $14.32^{a}$ & $13.53^{\mathrm{a}}$ & $14.32^{\mathrm{a}}$ & $12.65^{a}$ & $10.50^{a}$ & $14.09^{a}$ & $13.27^{\mathrm{a}}$ & & $12.16^{a}$ & $12.40^{\mathrm{a}}$ & $13.48^{\mathrm{a}}$ \\
\hline 1980 & : & $15.06^{\mathrm{a}}$ & $14.23^{a}$ & $13.25^{a}$ & & 15.67 & $15.25^{a}$ & $10.63^{a}$ & $13.47^{\mathrm{a}}$ & $14.55^{\mathrm{a}}$ & $13.12^{a}$ & $13.86^{\mathrm{a}}$ & $14.53^{\mathrm{a}}$ \\
\hline 1981 & : & $11.40^{\circ}$ & $16.02^{a}$ & $18.28^{a}$ & $16.44^{a}$ & $15.86^{\mathrm{a}}$ & 12.84 & 12.29 & $10.76^{a}$ & $12.30^{\mathrm{a}}$ & $10.56^{a}$ & $7.49^{a}$ & $7.94^{\mathrm{a}}$ \\
\hline 1982 & : & 20.97 & $15.04^{\circ}$ & 3.40 & $11.18^{\mathrm{a}}$ & $11.26^{\mathrm{a}}$ & $13.65^{\mathrm{a}}$ & $12.83^{a}$ & $6.62^{\mathrm{a}}$ & $12.99^{\mathrm{a}}$ & $13.54^{\mathrm{a}}$ & $12.62^{\mathrm{a}}$ & $14.23^{\mathrm{a}}$ \\
\hline 1983 & : & 13.00 & 9.30 & 14.18 & 13.93 & 14.75 & $12.55^{\mathrm{a}}$ & $13.16^{\mathrm{a}}$ & $0.20^{\mathrm{a}}$ & $14.12^{\mathrm{a}}$ & $14.31^{\mathrm{a}}$ & $14.28^{\mathrm{a}}$ & $14.43^{\mathrm{a}}$ \\
\hline 1984 & : & 14.43 & $14.71^{\mathrm{a}}$ & 14.74 & $14.84^{\mathrm{a}}$ & & 12.52 & 13.80 & 14.25 & 11.30 & 11.07 & $13.62^{\mathrm{a}}$ & $13.92^{\mathrm{a}}$ \\
\hline 985 & & $92^{a}$ & & & & & & & & & & & \\
\hline
\end{tabular}

REMARKS: Well discontinued, February 1985.

a pumping, b recently pumped, e estimated, $h$ tape measurement, J lowest water level recorded,

n nearty pumping well 


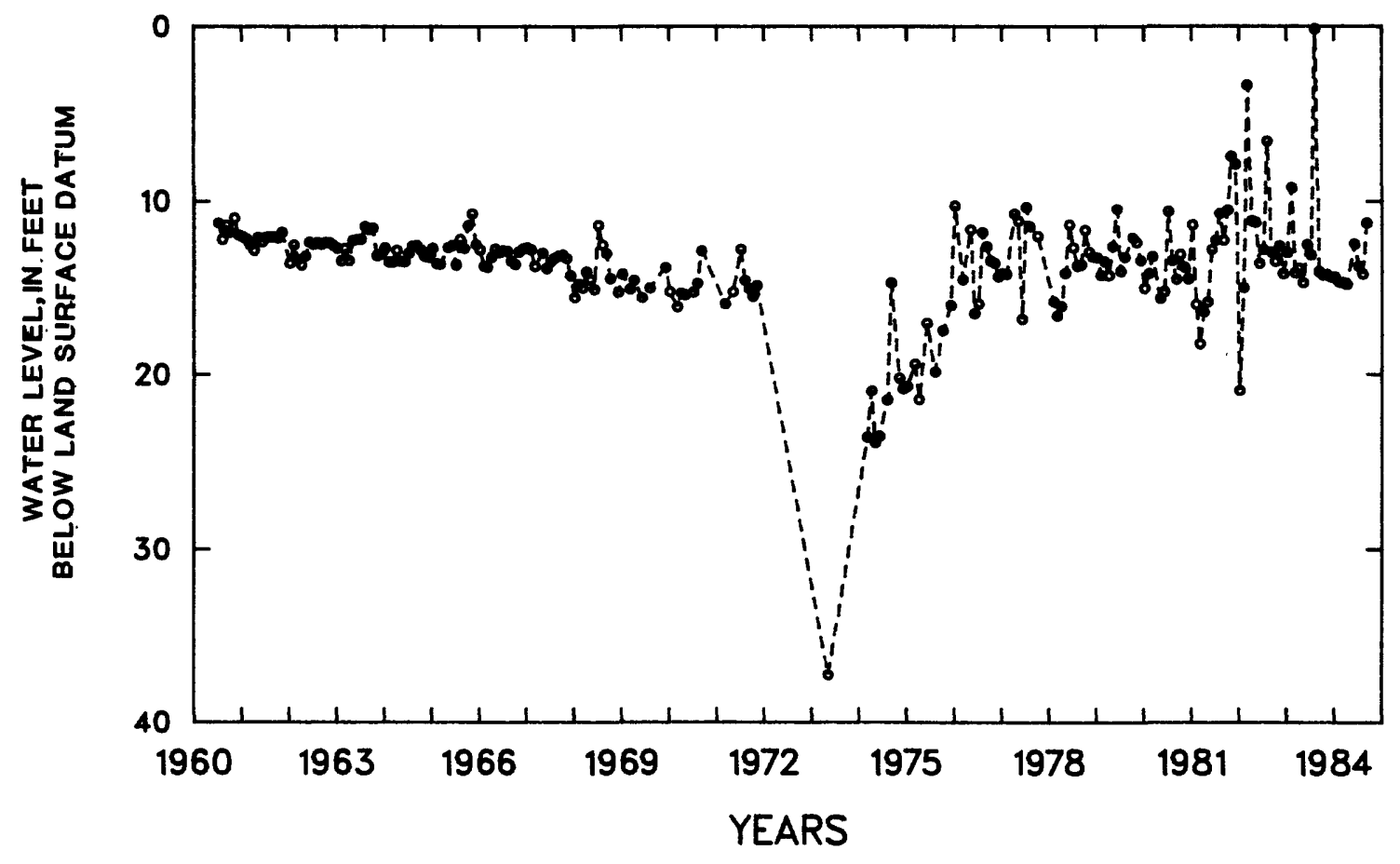

Figure 25.--Ground-water levels at Rosario,

local number 40 . 


\begin{tabular}{|c|c|c|c|c|c|c|c|c|c|c|c|c|c|}
\hline $\begin{array}{l}\text { LOCAL } \\
\text { WELL } \\
\text { LATIT } \\
\text { DIAME }\end{array}$ & $\begin{array}{l}\text { NUI } \\
\text { NAM } \\
\text { TUDE } \\
\text { STER }\end{array}$ & $\begin{array}{l}\text { IMBER : } \\
\text { IE : Ce } \\
: 18^{\circ} \\
:\end{array}$ & $\begin{array}{l}43 \\
\text { tra } 1 \\
10^{\prime} 18^{\prime \prime} \\
2 \text { inch }\end{array}$ & ela & $\begin{array}{l}\text { ELE } \\
\text { OWN } \\
\text { LON } \\
\text { DEP }\end{array}$ & $\begin{array}{l}\text { VATION } \\
\text { ER : Ma } \\
\text { GITUDE } \\
\text { TH : }(8\end{array}$ & $\begin{array}{l}\text { ABOVE M } \\
\text { yagüez } \\
: 67^{\circ} 09 \\
\text { feet }\end{array}$ & $\begin{array}{l}\text { EAN SEA } \\
\text { Sugar C } \\
\text { "17" } \\
\text { above }\end{array}$ & EVEL: & $1+$ & & & \\
\hline & : & & & & & MO & NTHS & & & & & & \\
\hline YEAR & : & JAN & FEB & MAR & APR & MAY & JUN & JUL & AUG & SEP & OCT & NOV & DEC \\
\hline 1959 & $:$ & & & & & & & & 0.24 & & & & \\
\hline 1960 & $\vdots$ & & & & & & & & & & 1.00 & -0.12 & 0.77 \\
\hline 1961 & $\vdots$ & 0.73 & 1.07 & 1.63 & 1.19 & 0.75 & 0.98 & 0.56 & 0.59 & -0.13 & 0.66 & -0.24 & \\
\hline 1962 & : & 0.60 & 1.17 & 1.67 & 1.72 & 1.19 & 0.69 & 1.22 & 1.24 & 0.89 & 0.98 & 1.16 & 1.46 \\
\hline 1963 & $\vdots$ & 1.81 & 1.78 & 1.36 & 1.29 & 1.31 & 0.50 & 0.46 & 0.04 & 0.37 & 0.00 & 0.53 & 0.87 \\
\hline 1964 & : & 1.35 & 1.86 & 1.93 & 2.07 & 1.35 & 1.28 & 0.60 & 0.52 & 0.60 & 0.44 & 0.84 & 1.02 \\
\hline 1965 & $\begin{array}{l}\text { : } \\
:\end{array}$ & 1.84 & 2.15 & 2.28 & 2.39 & 1.49 & 0.82 & 0.64 & 0.05 & 0.56 & -0.02 & 0.00 & 0.62 \\
\hline 1966 & $\vdots$ & 1.27 & 2.00 & 2.22 & 2.02 & 1.24 & 1.45 & 1.11 & 1.05 & 0.80 & 0.75 & 0.69 & 1.12 \\
\hline 1967 & : & 1.30 & 1.35 & 1.53 & 1.60 & 0.83 & 1.78 & 0.80 & 0.37 & 0.32 & 0.00 & 0.75 & 0.79 \\
\hline 1968 & $\begin{array}{l}\vdots \\
\vdots\end{array}$ & 1.43 & 2.18 & 2.62 & 2.70 & 1.93 & 0.30 & -0.32 & 0.16 & 0.03 & 0.23 & & 0.60 \\
\hline 1969 & $\vdots$ & 0.70 & & 1.49 & 2.03 & & 1.08 & & -0.11 & & & & 0.02 \\
\hline 1970 & $\vdots$ & -0.03 & & 0.00 & 1.39 & 1.59 & & 1.21 & 0.23 & -0.28 & & & \\
\hline 1971 & $\vdots$ & & & & & 0.03 & & -0.22 & -0.15 & -0.02 & 0.47 & & 0.11 \\
\hline 1972 & $\vdots$ & 0.75 & 0.68 & & 0.54 & 0.48 & & 0.55 & 0.48 & & -0.34 & & \\
\hline 1973 & : & 0.85 & & 0.90 & & 2.35 & 1.98 & 1.95 & 2.05 & & 0.36 & & 1.00 \\
\hline 1974 & : & & & 0.76 & 1.24 & 1.38 & 1.41 & & 0.64 & 0.10 & & 0.00 & 1.03 \\
\hline 1975 & : & 1.13 & & 0.56 & 1.37 & & 0.90 & & -0.12 & & -0.10 & -0.10 & 0.00 \\
\hline 1976 & $\vdots$ & 0.08 & & 0.66 & & 0.17 & & 0.50 & 0.61 & -0.24 & -0.03 & -0.10 & 0.00 \\
\hline 1977 & : & 0.20 & 0.10 & & 1.66 & 1.60 & 1.66 & & 0.00 & -0.18 & 0.00 & -0.38 & 0.00 \\
\hline 1978 & : & & -0.37 & 1.10 & 0.64 & 0.45 & 0.12 & -0.21 & -0.05 & -0.07 & -0.30 & -0.40 & -0.20 \\
\hline 1979 & $:$ & 0.46 & 0.56 & 1.10 & 1.57 & 0.58 & -0.30 & -0.27 & -0.35 & & -0.47 & -0.50 & -0.37 \\
\hline 1980 & $\vdots$ & 0.38 & 0.30 & 0.19 & & 0.15 & 0.00 & 0.00 & 0.35 & 0.00 & & 0.00 & 0.00 \\
\hline 1981 & $:$ & 0.00 & 0.00 & -0.16 & 0.04 & 0.41 & -0.36 & 0.00 & 0.00 & 0.00 & 0.00 & 0.00 & 0.00 \\
\hline 1982 & : & 0.00 & -0.05 & -0.24 & 0.16 & 0.00 & -0.13 & 0.00 & -0.50 & -0.49 & -0.48 & 0.00 & 0.00 \\
\hline 1983 & $\vdots$ & 0.00 & 0.00 & -0.40 & 0.08 & -0.20 & 0.00 & 0.00 & 0.00 & 0.00 & -0.50 & -0.53 & -0.53 \\
\hline 1984 & $:$ & -0.45 & -0.55 & -0.42 & -0.47 & 0.22 & -0.59 & -0.24 & 0.53 & -0.58 & -0.60 & -0.57 & -0.57 \\
\hline & $\vdots$ & - & & & & & & & & & & & \\
\hline
\end{tabular}

REMARKS: Well discontinued, February 1985.

a pumping, b recently pumped, e estiaated, $h$ tape aeasurement, $j$ lowest water level recorded, n nearby pupping well 


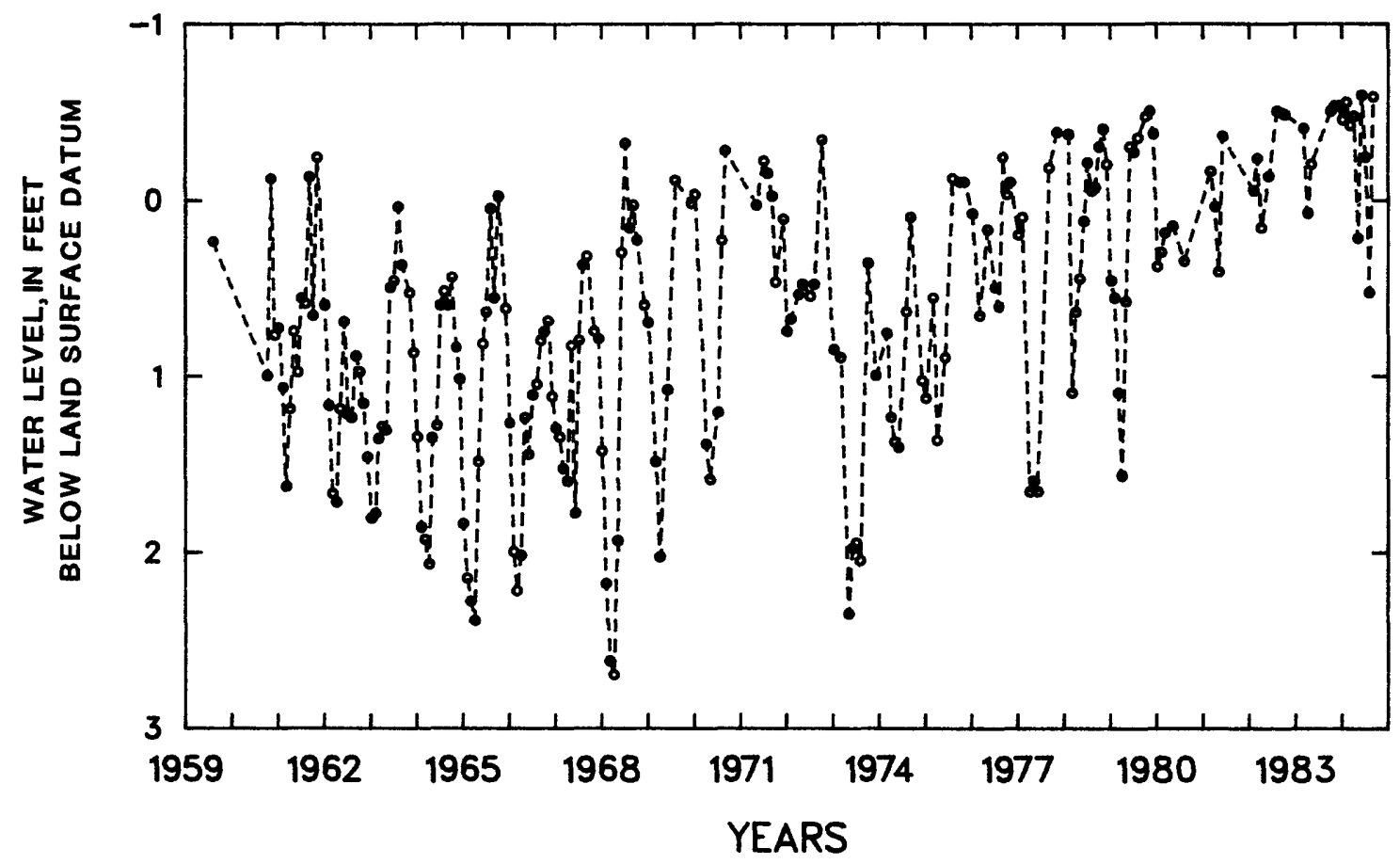

Figure 26.--Ground-water levels at Central Rochelalse, local number 43. 
WELLS IN THE

WEST COAST PROVINCE

Río Yaguez Valley

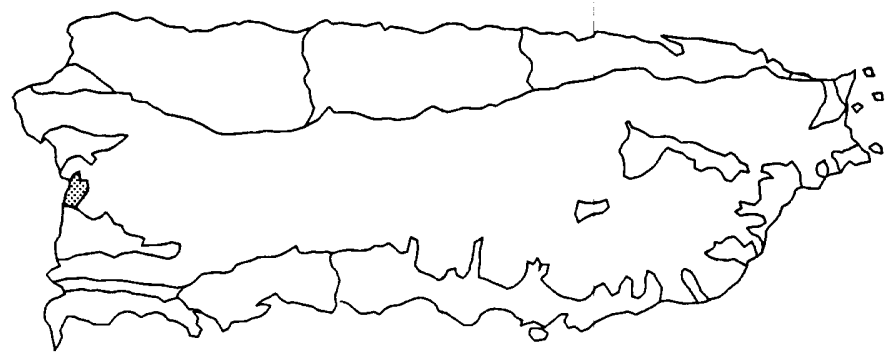

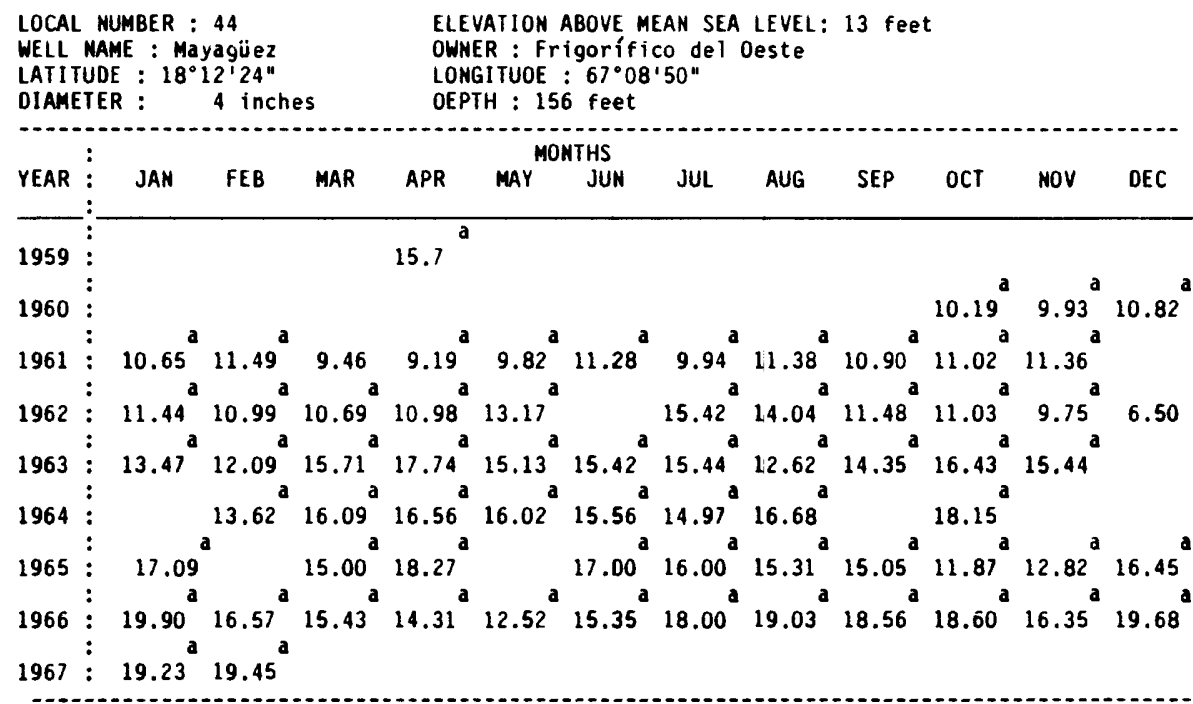

REMARKS: Well discont inued, March 1967.

pumping, b recently pumped, e estimated, $h$ tape measurement, $j$ lowest water level recorded,

n nearby pumping well 


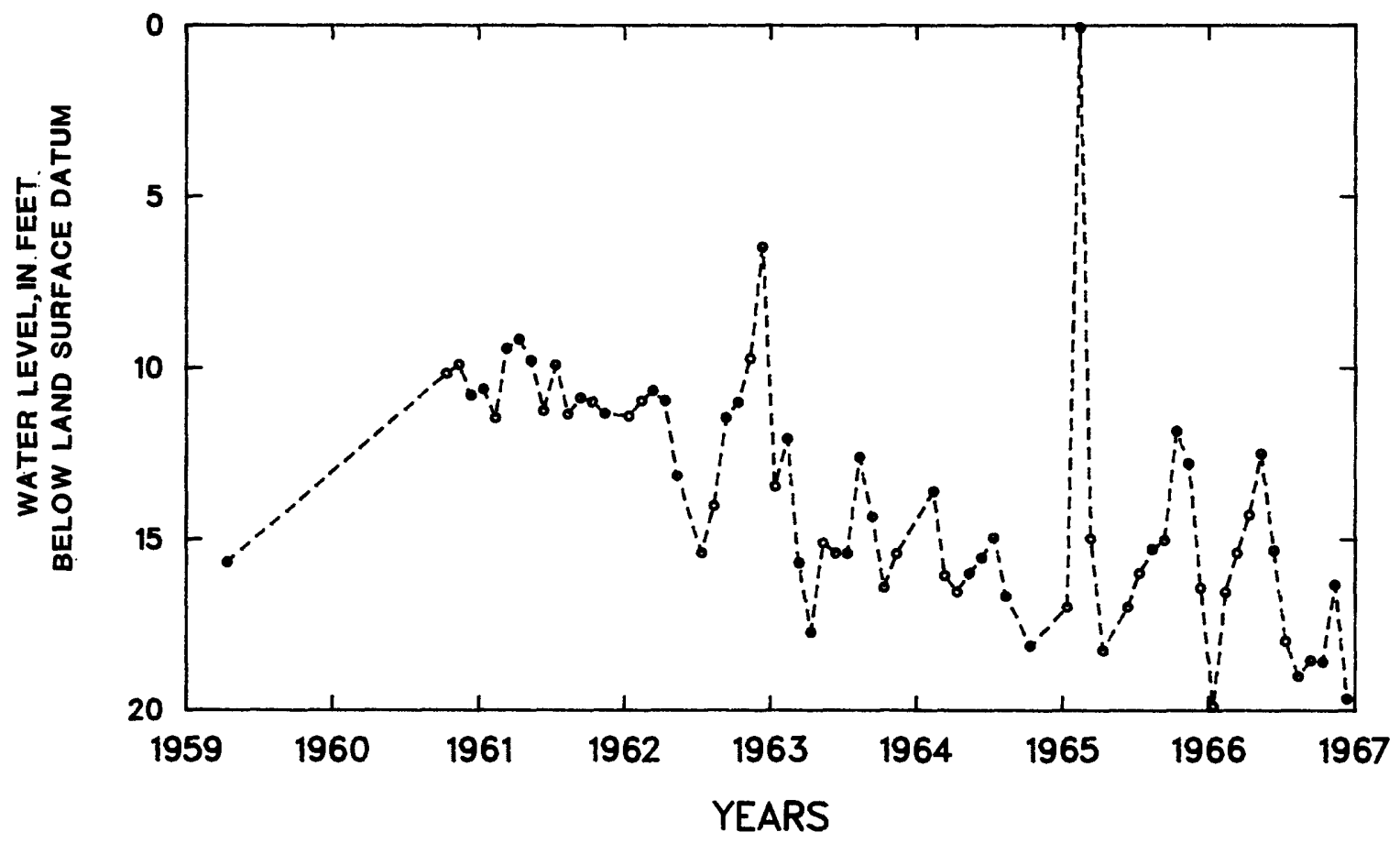

Figure 27 .--Ground-water levels at Mayagüez, local number 44. 


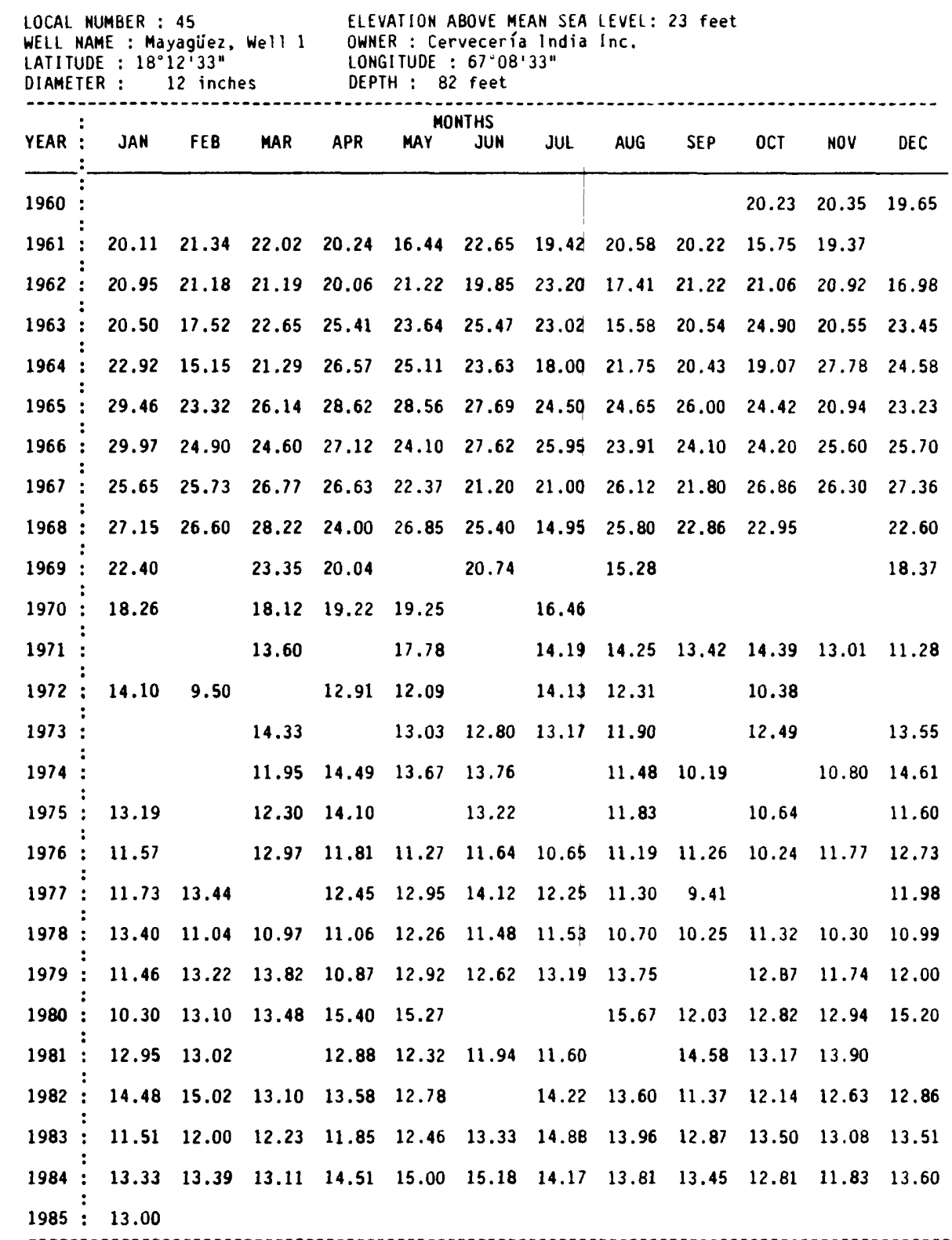

REMARKS: Well discont inued, February 1985.

a pumping, b recently pumped, e estimated, $h$ tape measurement, $j$ lowest water level recorded,

nearby pumping well 


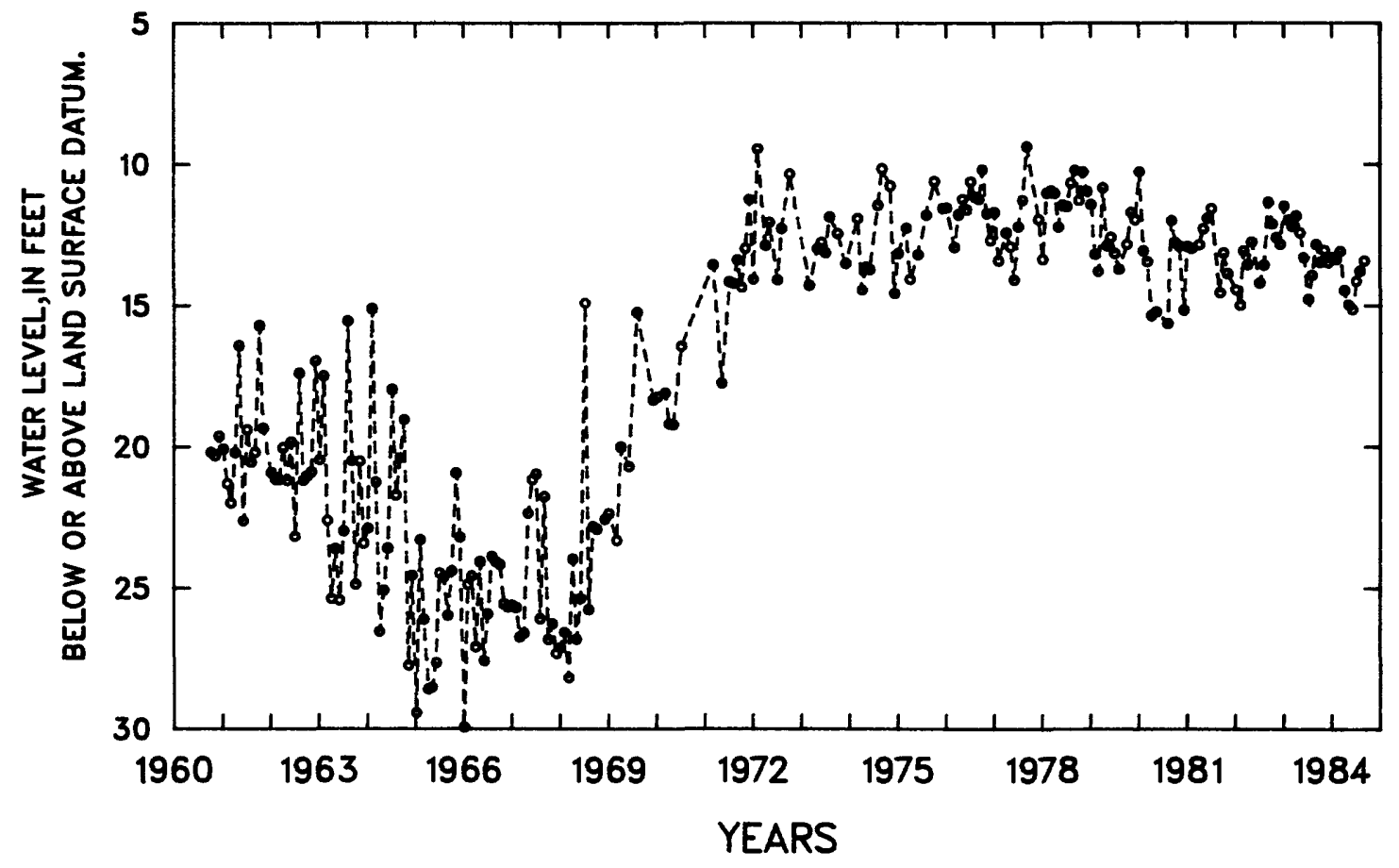

Figure 28,--Ground-water levels at Well 1 Mayaguiez, local number 45. 


\section{WELLS IN THE \\ WEST COAST PROVINCE \\ Río Grande de Añasco Valley}

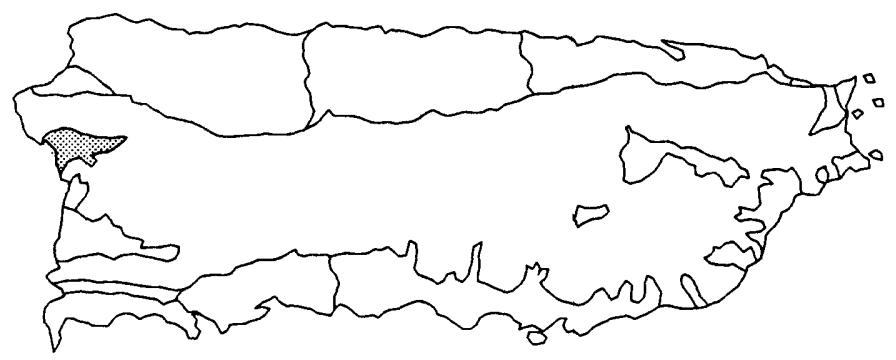

\begin{tabular}{|c|c|c|c|c|c|c|c|c|c|c|c|c|c|}
\hline $\begin{array}{l}\text { LOCAL } \\
\text { WELL } \\
\text { LATIT } \\
\text { DIAME }\end{array}$ & $\begin{array}{l}\text { NU } \\
\text { NAM } \\
\text { UDE } \\
\text { TER }\end{array}$ & $\begin{array}{l}\text { MBER : } \\
\text { E: Ma } \\
: 18^{\circ} \\
:\end{array}$ & $\begin{array}{l}53 \\
\text { aguiez } \\
5^{\prime} 22^{\prime \prime} \\
8 \text { inch }\end{array}$ & $\begin{array}{l}\text { irport } \\
\text { s }\end{array}$ & $\begin{array}{l}\text { ELE } \\
\text { OWH } \\
\text { LON } \\
\text { DEP }\end{array}$ & $\begin{array}{l}A T I O N \\
R: P \\
\text { R P } \\
\text { ITUDE } \\
H: 35\end{array}$ & $\begin{array}{l}\text { BOVE M } \\
\text { Port } \\
67^{\circ} 09 \\
\text { feet }\end{array}$ & $\begin{array}{l}\text { AN SEA } \\
\text { Autho } \\
09^{\prime \prime}\end{array}$ & $\begin{array}{l}\text { LEVEL } \\
\text { ity }\end{array}$ & $20 \mathrm{fe}$ & & & \\
\hline & : & & & & & MO & & & & & & & \\
\hline YEAR & $\vdots$ & JAN & FEB & MAR & APR & MAY & JUN & JUL & AUG & SEP & OCT & NOV & DEC \\
\hline 1960 & : & & & & & & & & & & 3.45 & 4.34 & 4.52 \\
\hline 1961 & : & & 4.52 & & $5.4 B$ & & & & & & 3.03 & & \\
\hline 1962 & : & & & & 7.84 & & & & & & 4.24 & & \\
\hline & : & & & & & & & & & & & & \\
\hline 1963 & : & & & & 7.92 & & & & & & 2.18 & & 3.20 \\
\hline & : & & & & & & & & & & & & \\
\hline 1964 & : & & & & 7.51 & & & & & & 2.77 & & \\
\hline 1965 & : & & & & 7.58 & & & 3.00 & & & 2,50 & & \\
\hline 1966 & : & & & 6.27 & 6.32 & & & & & 6.30 & 6.35 & & \\
\hline & $\vdots$ & & & & & & & & & & & & \\
\hline 1967 & : & & & 6.32 & 6.27 & 5.95 & 5.93 & 3.42 & 3.63 & 3.26 & 4.15 & 4.00 & 4.54 \\
\hline 1968 & $\vdots$ & 5.30 & 6.74 & $7.2 B$ & 7.43 & 6.47 & 3.80 & $2.4 B$ & 2.58 & 2.60 & 3.42 & & 3.30 \\
\hline 1969 & $\vdots:$ & 2.50 & & 6.22 & 4.98 & & 4.07 & & $2.3 \mathrm{~B}$ & & & & 3.49 \\
\hline 1970 & $:$ & & & & & & & 5.34 & 3.58 & 2.15 & & & \\
\hline & : & & & & & & & & & & & & \\
\hline 1971 & : & & & 6.78 & & & & 4.25 & 3.17 & 2.84 & 2.22 & & 1.64 \\
\hline 1972 & : & & & & & & & & 4.20 & & 1.58 & & \\
\hline 1973 & : & 0.85 & & 0.90 & & 2.35 & 1.98 & 1.95 & 2.05 & & 0.36 & & 1.00 \\
\hline 1974 & : & & & 0.76 & 1.24 & 1.38 & 1.41 & & 0.64 & 0.10 & & -0.00 & 1.03 \\
\hline 1975 & : & 1.13 & & 0.56 & 1.37 & & 0.90 & & -0.12 & & -0.10 & -0.10 & -0.00 \\
\hline 1976 & : & 0.08 & & 0.66 & & 0.17 & & 0.50 & 0.61 & -0.24 & & 2.31 & 3.10 \\
\hline 1977 & : & 3.77 & 5.22 & & 6.99 & 7.07 & 6.22 & 4.61 & & 4.50 & 6.74 & 2.15 & 3.29 \\
\hline 1978 & $\vdots$ & 5.30 & 4.30 & 5.56 & 5.83 & 5.48 & 4.76 & 2.90 & 2.87 & 2.25 & 0.96 & 2.26 & 3.48 \\
\hline 1979 & : & 4.71 & 8.70 & 5.00 & 6.60 & 5.10 & 2.63 & 2.09 & ' 2.38 & & 1.74 & 7.60 & 2.93 \\
\hline & : & & & & & & & & & & & & \\
\hline 1980 & : & 4.35 & 4.64 & 5.27 & 5.59 & 4.90 & 2.66 & 2.49 & 2.83 & 2.12 & 1.45 & 2.58 & 3.54 \\
\hline 1981 & : & 4.14 & 2.85 & & 3.85 & 3.05 & 2.04 & 2.15 & & 2.07 & 2.16 & 2.23 & \\
\hline 1982 & : & 4,19 & 5.28 & 4.60 & 6.60 & 5.25 & & 5.69 & 4.93 & 4.38 & 5.58 & 5.40 & 5.05 \\
\hline & : & & & & & & & & & & & & \\
\hline 1983 & : & 5.90 & 5.10 & 5.48 & 5.85 & 5.42 & 3.88 & 4.63 & 2.10 & 2.00 & 2.62 & 2.07 & 2.78 \\
\hline 1984 & : & 3.97 & 4.72 & 4.68 & 5.55 & 6.09 & 3.04 & 2.32 & 2.42 & 2.04 & 1.93 & & \\
\hline
\end{tabular}

REMARKS: We11 discont inued, November 1984.

a pumping, b recently pumped, e estimated, $h$ tape measurenent, $j$ lowest water level recorded, n nearby pumping well 


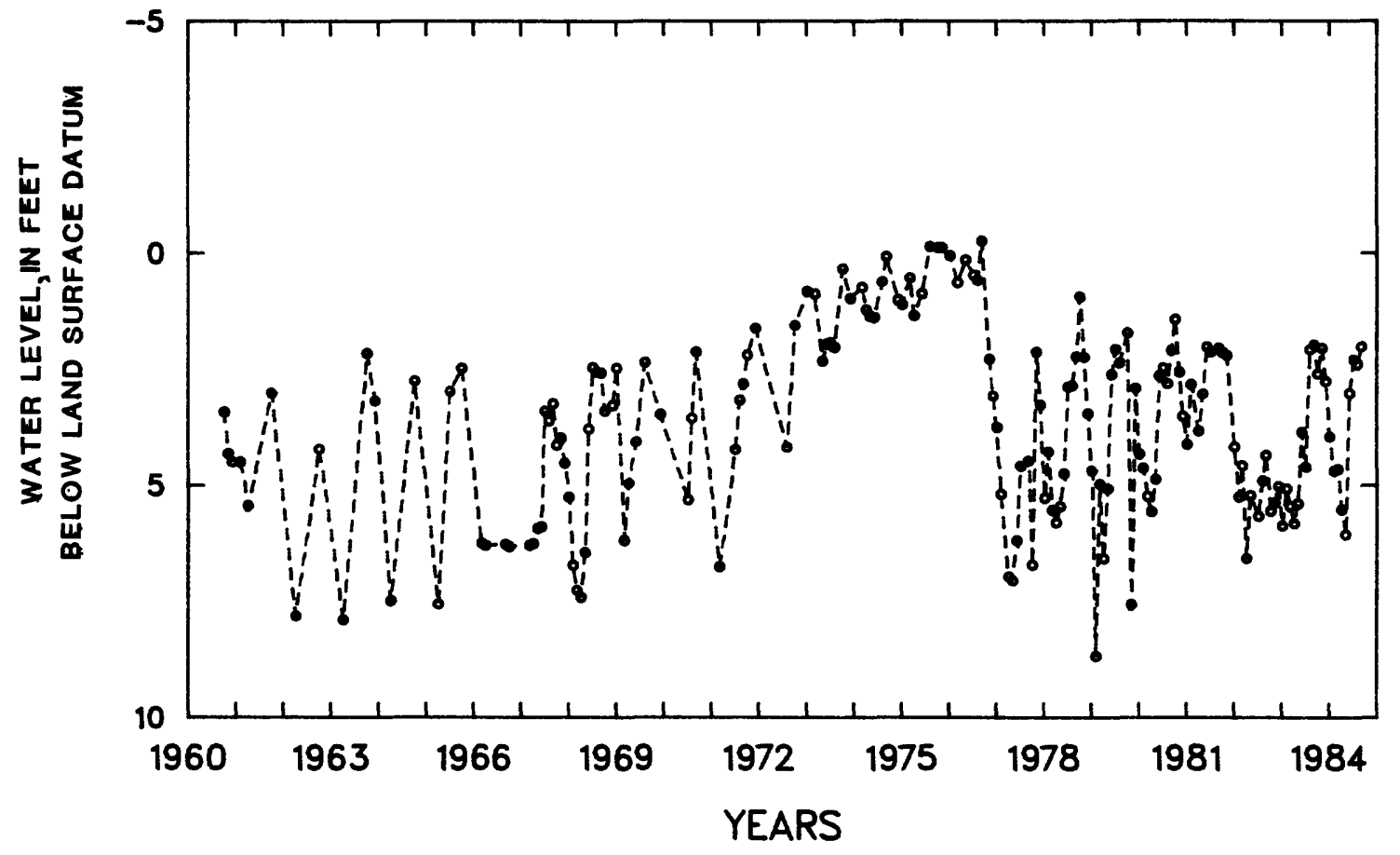

Figure 29.--Ground-water levels at Mayagüez Airport, local number 53. 
WELLS IN 'THE

WEST COAST PROVINCE

Afo Culebrina Valley

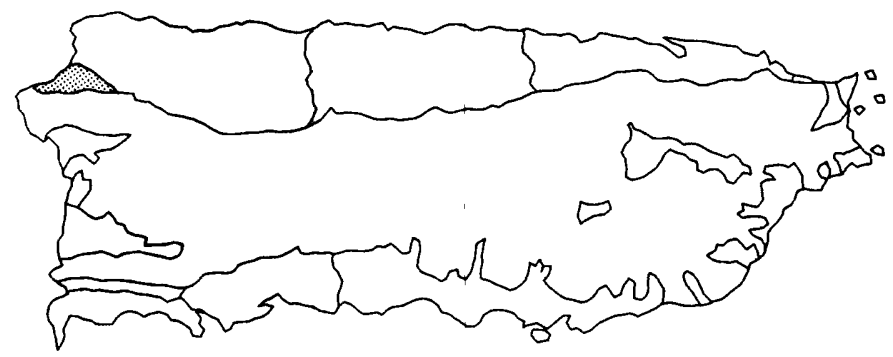

\begin{tabular}{|c|c|c|c|c|c|c|c|c|c|c|c|c|}
\hline $\begin{array}{l}\text { LOCAL } \\
\text { WELL } \\
\text { LATITL } \\
\text { DIAME }\end{array}$ & $\begin{array}{l}\text { NUMBER : } \\
\text { VAME : Agu } \\
\text { JDE : } 18^{\circ} 2 \\
\text { IER : } 12-2\end{array}$ & $\begin{array}{l}58 \\
\text { Jada } \\
22^{\prime} 28^{\prime \prime} \\
20 \text { inch }\end{array}$ & & $\begin{array}{l}\text { ELE } \\
\text { OWN } \\
\text { LON } \\
\text { DEP }\end{array}$ & $\begin{array}{l}\text { VATION } \\
\text { ER : P. } \\
\text { GI TUDE } \\
\text { TH : } 16\end{array}$ & $\begin{array}{l}\text { ABOVE } \\
R \text { R. Aque } \\
: 67^{\circ} 11 \\
0 \text { feet }\end{array}$ & $\begin{array}{l}\text { EAN SE } \\
\text { duct } \\
\text { ' } 33 \text { " }\end{array}$ & $\begin{array}{l}\text { VEL: } \\
\text { ewer }\end{array}$ & A & & & \\
\hline & * & & & & MO & NTHS & & & & & & \\
\hline YEAR & JAN & FEB & MAR & APR & MAY & JUN & JUL & AUG & SEP & OCT & NOV & DEC \\
\hline 160 & 3.12 & 2.98 & 4.32 & 3.83 & 1.18 & 1.44 & & 1.53 & 1.47 & 1.77 & $54.62^{b}$ & 20.81 \\
\hline 1961 & 16.22 & 28.08 & $83.16^{\mathrm{a}}$ & 85.4 & $84.9^{a}$ & $84.3^{a}$ & $67.9^{a}$ & $72.8^{a}$ & $67.04^{a}$ & B3. $78^{\mathrm{a}}$ & $60.90^{a}$ & \\
\hline 1962 & : $\quad 57.66$ & $81.53^{\mathrm{a}}$ & 33.20 & 21.10 & $64.84^{\mathrm{a}}$ & 15.35 & 8.89 & 5.85 & 4.67 & 4.56 & 4.35 & 18.84 \\
\hline 1963 & $: \quad 12.91$ & 9.77 & 28.35 & $60.85^{a}$ & 29.54 & 29.48 & 16.48 & 25.65 & $72.85^{\mathrm{a}}$ & 47.61 & $B 3.66^{a}$ & 43.75 \\
\hline 1964 & 46.60 & $87.58^{a}$ & 49.39 & 35.15 & $\mathrm{~B} 1.40^{\mathrm{a}}$ & 39.71 & $84.90^{\circ}$ & $86.00^{a}$ & $83.90^{\circ}$ & $76.00^{a}$ & $78.00^{\circ}$ & $83.20^{\mathrm{a}}$ \\
\hline 1965 & $: \quad 72.80^{\mathrm{a}}$ & $79.30^{a}$ & $68.30^{\mathrm{a}}$ & & 51.65 & 29.78 & 12.40 & 8.17 & 6.67 & 5.73 & 5.60 & 4.47 \\
\hline 1966 & $\quad 40.28^{\circ}$ & 6.52 & 7.41 & 8.08 & 7.93 & $B 0.25^{\mathrm{a}}$ & 44.36 & $72.40^{\circ}$ & 65.24 & 65.40 & 10.04 & 8.83 \\
\hline 1967 & 10.22 & 10.30 & 8.15 & 8.00 & B. 33 & 12.41 & 7.39 & 3.91 & 3.34 & 4.12 & 4.36 & 5.64 \\
\hline 1968 & 2.47 & 5.80 & 6.06 & 8.04 & 7.56 & 3.18 & 3.65 & 2.14 & $82.80^{\mathrm{a}}$ & $81.90^{d}$ & & $\mathrm{~B} 1.70^{\circ}$ \\
\hline 1969 & 6.90 & & 11.70 & & & 16.27 & & & & $89.00^{\circ}$ & 49.43 & \\
\hline 1970 & : & 84.12 & & & & & & & & & & \\
\hline 1971 & : & & & & $84.85^{a}$ & & $66.04^{a}$ & $71.03^{a}$ & $70.81^{a}$ & 62.32 & $60.17^{a}$ & \\
\hline 1972 & $: \quad 44.15$ & $66.53^{a}$ & & $2 B .85$ & 29.58 & & 30.82 & 51.35 & & $82.10^{\mathrm{a}}$ & $71.70^{\mathrm{a}}$ & \\
\hline 1973 & : & & & & 62.98 & $47.54^{a}$ & & 53.03 & & & & \\
\hline 1974 & : & & 3.52 & 4.18 & $82.23^{a}$ & $82.42^{a}$ & & & 7.56 & & 10.29 & 3.56 \\
\hline 1975 & 3.17 & & 2.46 & 2.90 & & 1.20 & & -0.30 & -0.81 & -0.85 & & -0.30 \\
\hline 1976 & 0.44 & & 3.49 & 3.31 & 2.22 & 0.92 & 0.81 & 2.27 & 0.80 & 0.88 & 1.80 & 3.04 \\
\hline 1977 & 3.65 & 3.45 & 4.00 & 4.35 & 4.57 & 3.70 & 1.10 & 0.80 & 0.58 & 0.64 & 0.50 & 0.93 \\
\hline 1978 & 2.50 & 2.25 & 2.83 & 2.97 & 2.22 & 1.10 & 0.08 & & 0.35 & -0.02 & 1.17 & \\
\hline 1979 & 3.28 & & & & & & & & & & 0.75 & 1.18 \\
\hline 1980 & 2.44 & 2.20 & & 3.94 & 2.37 & 0.85 & -0.12 & 0.50 & -0.09 & & 0.38 & 1.59 \\
\hline 1981 & 1.89 & 1.61 & 2.27 & 2.29 & 1.89 & -0.23 & 0.05 & 0.20 & 0.40 & 0.38 & -0.08 & 0.34 \\
\hline 1982 & 0.01 & 0.58 & 1.78 & 2.35 & 0.49 & 0.26 & -0.09 & 0.11 & -0.12 & 0.40 & 0.27 & 1.34 \\
\hline 1983 & 1.20 & 2.20 & 3.05 & 3.83 & 1.80 & 0.89 & 1.35 & 1.58 & 0.58 & 0.38 & 0.70 & 1.64 \\
\hline 1984 & 2.49 & 2.90 & 2.13 & 2.76 & 3.33 & 1.55 & 0.12 & 1.24 & 1.11 & & & \\
\hline
\end{tabular}

a pumping, b recently pumped, e estimated, $h$ tape measurement, $j$ lowest water level recorded, n nearby puaping well 


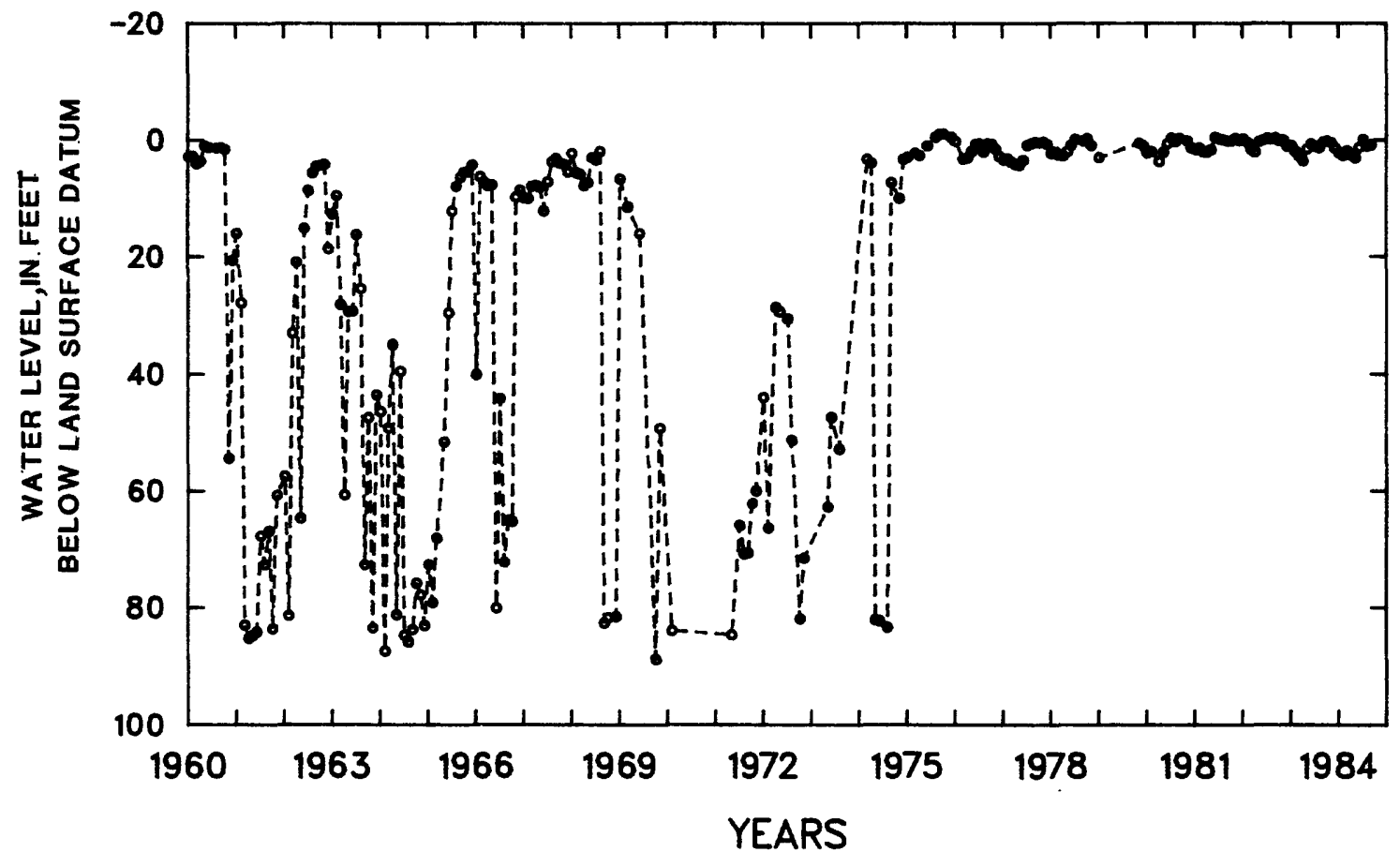

Figure 30.--Ground-water levels at Aguada, local number 58. 
WELLS IN THE

EAST COAST PROVINCE

Fajardo
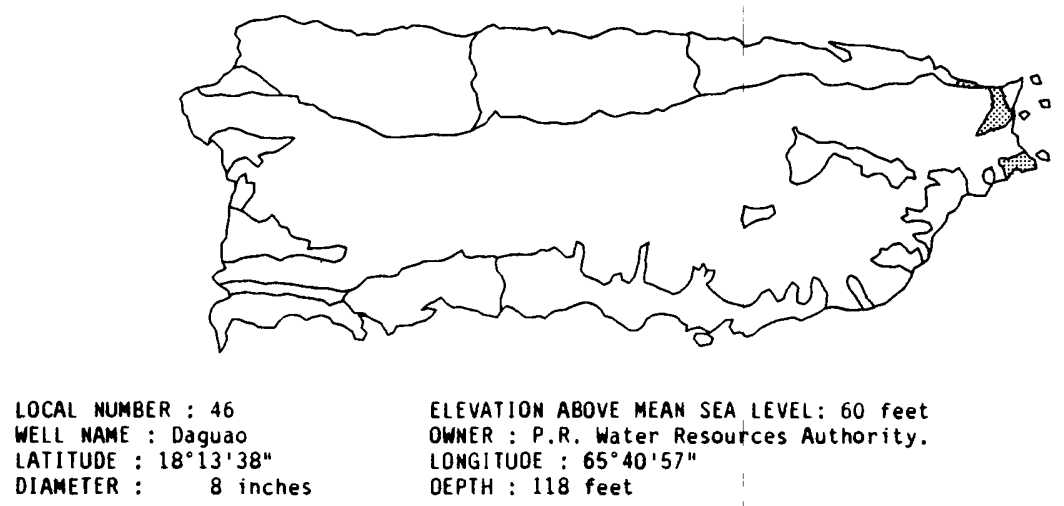

ELEVATION ABOVE MEAN SEA LEVEL: 60 feet

OWNER : P.R. Water Resources Authority,

LONGITUOE : $65^{\circ} 40^{\prime} 57^{\prime \prime}$

OEPIH : 118 feet

\begin{tabular}{|c|c|c|c|c|c|c|c|c|c|c|c|c|c|}
\hline \multirow[b]{2}{*}{ YEAR } & \multirow[t]{2}{*}{$:$} & \multirow[b]{2}{*}{ JAN } & \multirow[b]{2}{*}{ FEB } & \multirow[b]{2}{*}{ MAR } & \multirow[b]{2}{*}{ APR } & \multicolumn{2}{|c|}{ MONTHS } & \multirow[b]{2}{*}{ JUL } & \multirow[b]{2}{*}{ AUG } & \multirow[b]{2}{*}{ SEP } & \multirow[b]{2}{*}{$\mathrm{OCT}$} & \multirow[b]{2}{*}{ NOV } & \multirow[b]{2}{*}{ OEC } \\
\hline & & & & & & MAY & JUN & & & & & & \\
\hline 1961 & : & & & & & & 4.09 & & & 3.68 & 2.48 & 2.97 & 1.83 \\
\hline 1962 & : & 2.42 & 2.36 & 3.39 & 3.25 & 3.08 & 1.89 & 2.31 & 2.58 & 1.70 & 1.93 & 2.05 & 2.50 \\
\hline 1963 & : & 2.80 & 2.70 & 3.78 & 2.78 & 3.02 & 3.05 & 3.34 & 3.07 & 2.31 & 3.56 & 3.64 & 3.03 \\
\hline 1964 & : & 3.34 & 4.35 & 5.60 & & 5.67 & 6.33 & & 5.38 & 5.44 & 5.58 & 5.41 & 4.49 \\
\hline 1965 & $:$ & 5.17 & 5.23 & 5.69 & & & & & & & & & \\
\hline
\end{tabular}

REMARKS: Well destroyed, March 1965.

a pumping, b recently pumped, e estimated, $h$ tape measurement, $j$ lowest water level recorded,

n nearby pumping well

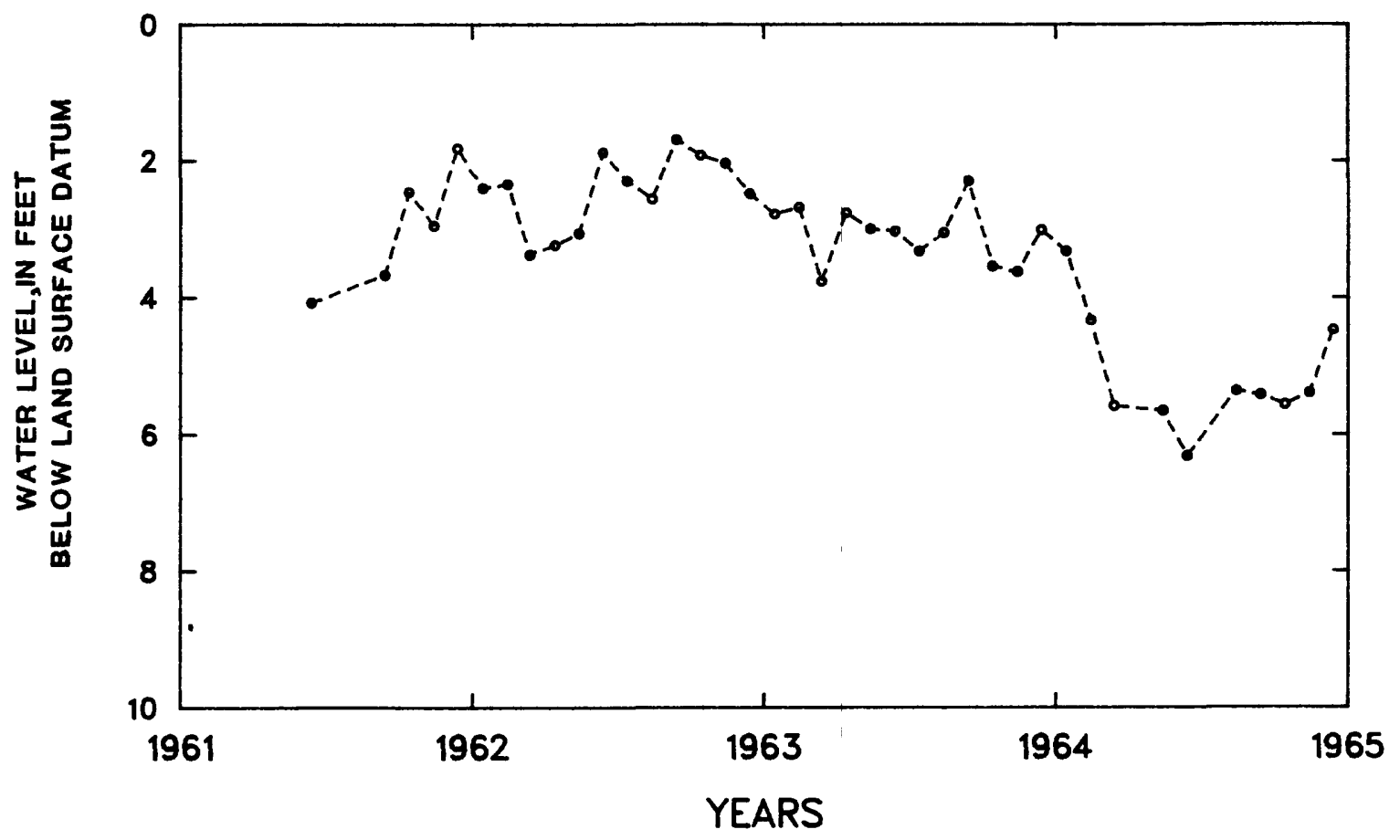

Figure 31.--Ground-water levels at Daguao, local number 46. 
WELLS IN THE

EAST COAST PROVINCE

Naguabo-Humacao

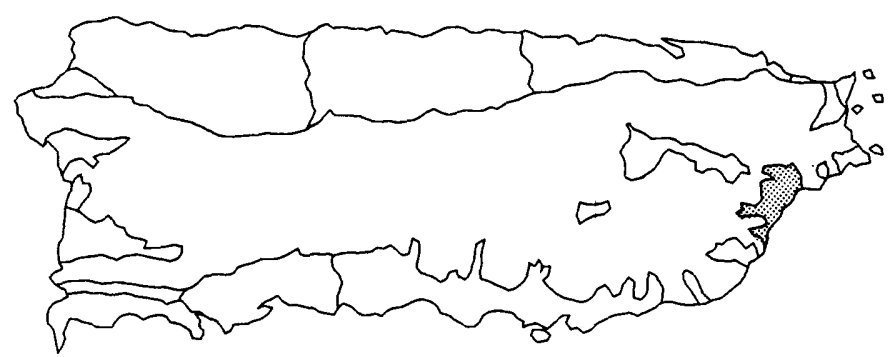

LOCAL NUMBER : 172

ELEVATION ABOVE MEAN SEA LEVEL: 57.40 feet

WELL NAME : Río Humacao GW Sta. OWNER: U.S.G.S., City of Humacao

LATITUDE : 1 B $^{\circ} 08^{\prime} 50^{\prime \prime}$

OWNER : U.S.G.S., City of Humacao

DIAMETER : $2-4$ inches

DEPTH : 43 feet

\begin{tabular}{rllllllllllllll} 
YEAR & JAN & FEB & MAR & APR & MAY MONTHS & JUN & JUL & AUG & SEP & OCT & NOV & DEC \\
\hline 1983 & $\vdots$ & & & & & & & & & & & 17.70 & 17.76 \\
1984 & $\vdots$ & 17.71 & 17.84 & 18.03 & 18.17 & 18.22 & 17.70 & 17.37 & 17.49 & 17.24 & 17.16 & 17.25 & 17.63 \\
1985 & 17.73 & 18.01 & 17.95 & 17.97 & 18.07 & 17.95 & WELL & OISCONTINUED. & & & &
\end{tabular}

REMARKS: Well discontinued, July, 1985

a pumping, b recently pumped, e estimated, $h$ tape neasurement, $j$ lowest water level recorded,

n nearby pumping well

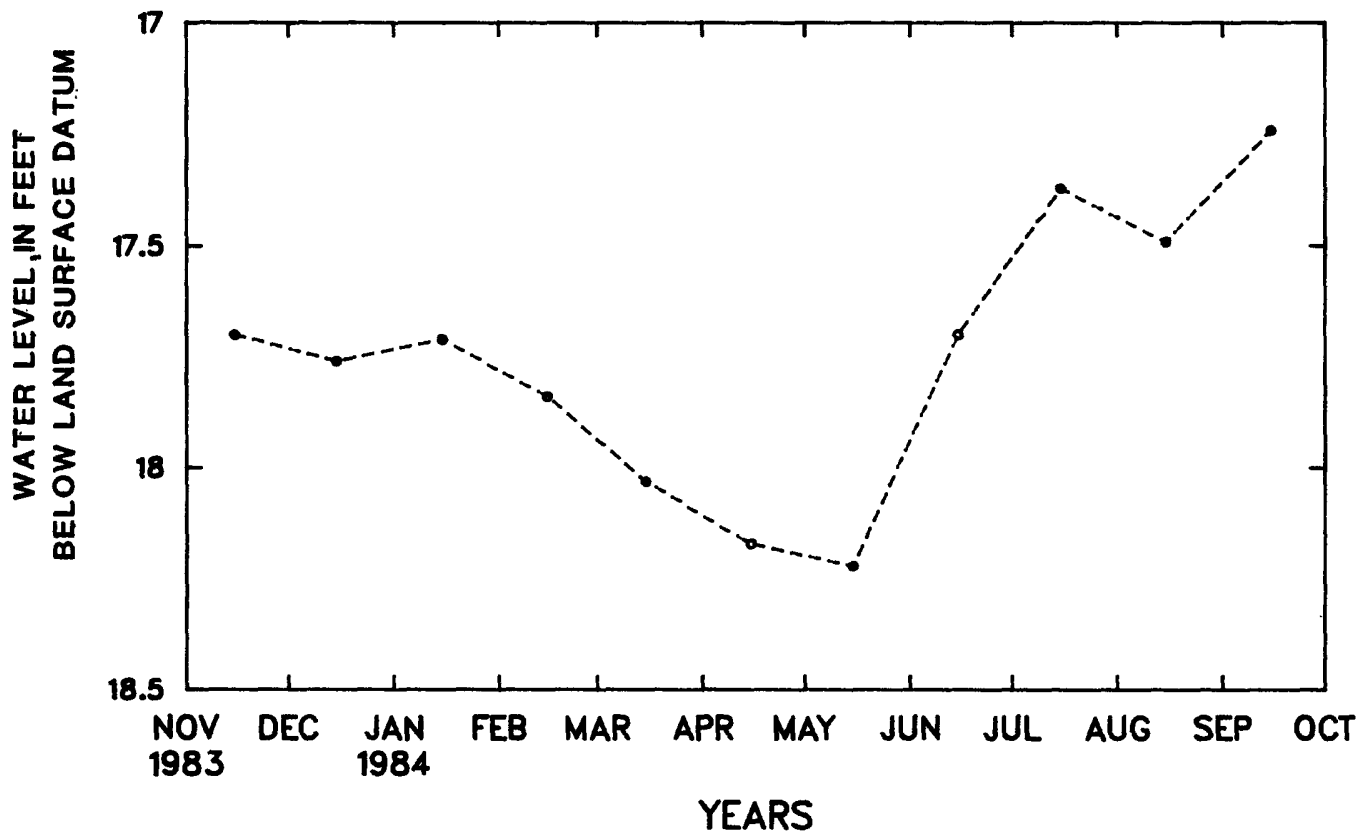

Figure 32.--Ground-water levels at Río Humacao Ground Water Station, local number 172. 


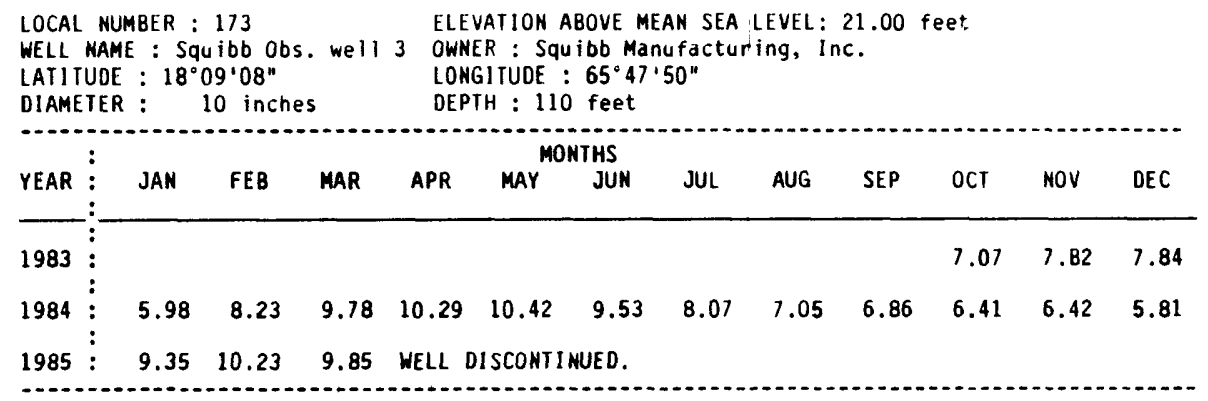

REMARKS: Well discont inued, April, 1985

a pumping, b recently pumped, e estimated, $h$ tape measurement, $j$ lowest water level recorded n nearby pumping well

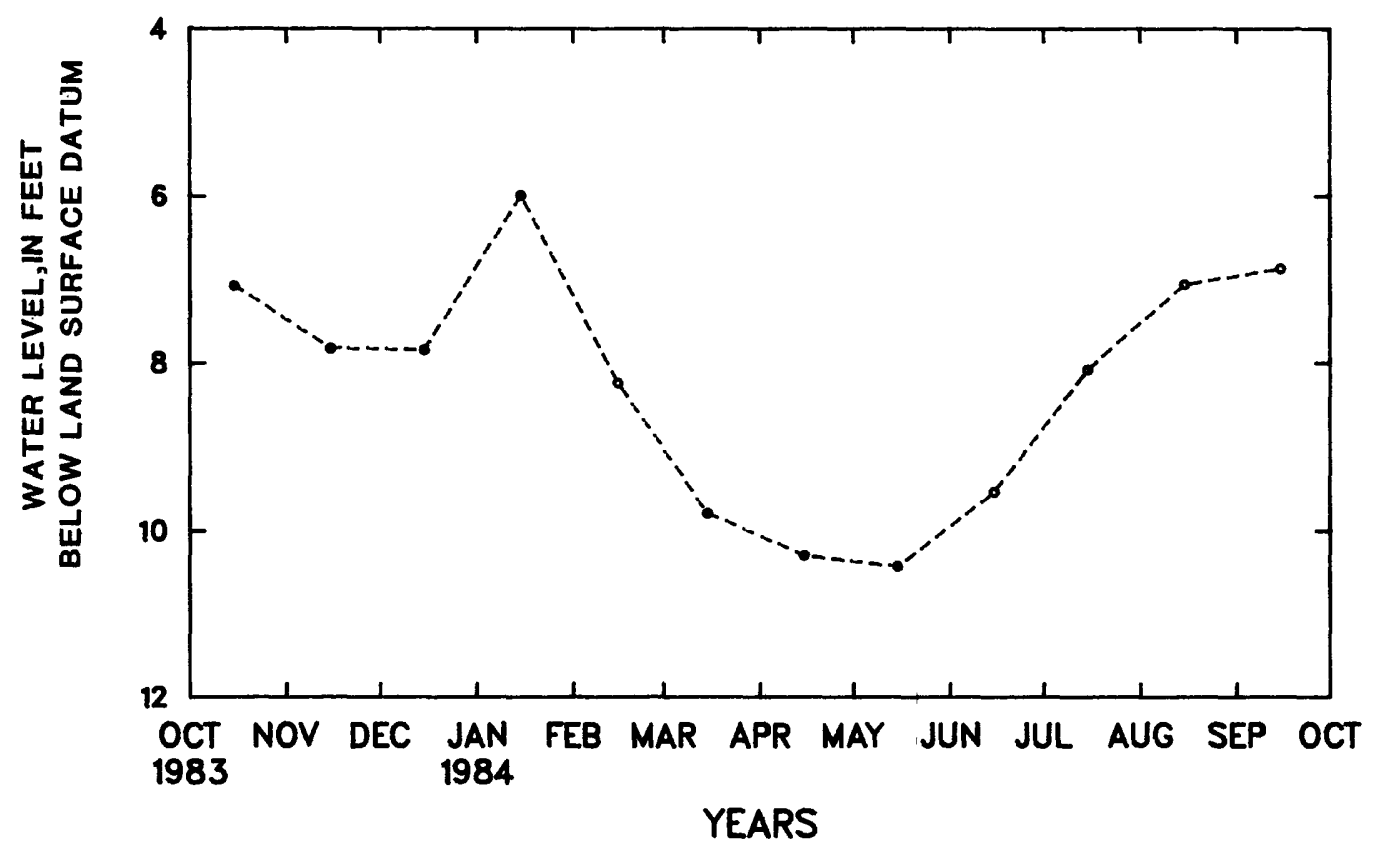

Figure 33.--Ground-water levels at Squibb Observation well number 3 , local number 173. 
LOCAL NUMBER : 171

ELEVATION ABOVE MEAN SEA LEVEL: 19.10 feet

WELL NAME : Arroyo Well No. 1 OWNER : Carlos Arroyo and Roberto Ramírez

LATITUOE : $18^{\circ} 12^{\prime} 17^{\prime \prime}$

LONGITUOE : $65^{\circ} 45^{\prime} 30^{\circ}$

OIAMETER: $4-2$ inches DEPTH : 32 feet

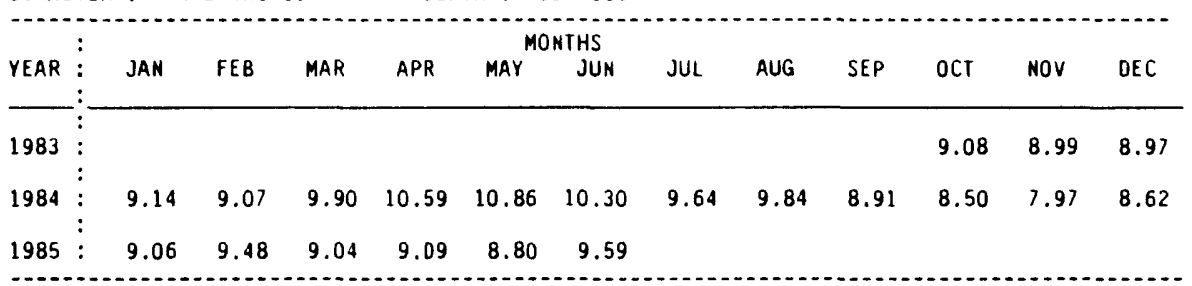

REMARKS: Well discontinued, July 1985

a pumping, b recently pumped, e estimated, $h$ tape measurement, $j$ lowest water level recorded,

n nearby pumping well 


\section{WELLS IN THE \\ EAST COAST PROVINCE \\ Yabucoa}

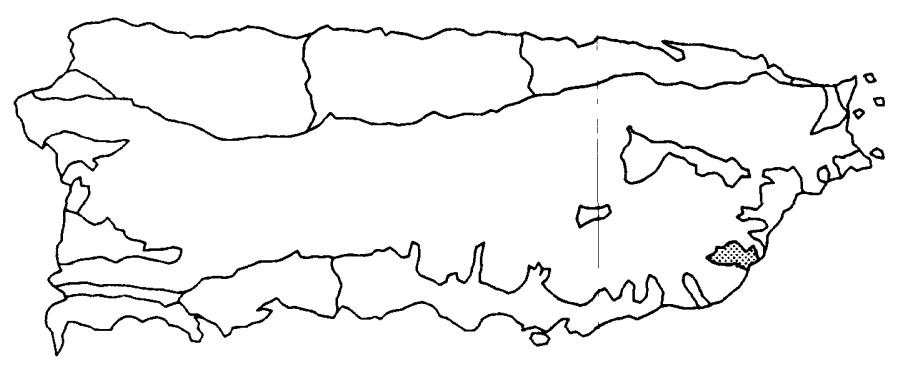

\begin{tabular}{|c|c|c|c|c|c|c|c|c|c|c|c|c|}
\hline $\begin{array}{l}\text { LOCAL } \\
\text { WELL } \\
\text { LATIT } \\
\text { DIAME }\end{array}$ & $\begin{array}{l}\text { MUMBER : } \\
\text { NAME : Ya } \\
\text { TUDE : } 18^{\circ} \\
\text { ETER : }\end{array}$ & $\begin{array}{l}95 \\
\text { bucoa } 1 \\
03^{\prime} 27^{\prime \prime} \\
6 \text { inch }\end{array}$ & or Tr & $\begin{array}{l}\text { ELE } \\
\text { OWN } \\
\text { LOM } \\
\text { DEP }\end{array}$ & $\begin{array}{l}\text { VATION } \\
\text { ER : P } \\
\text { GI TUDE } \\
\text { TH : } 12\end{array}$ & $\begin{array}{l}\text { ABOvE } \\
\text { R. Aque } \\
: 65^{\circ} 51 \\
0 \text { feet }\end{array}$ & $\begin{array}{l}\text { EAN SEA } \\
\text { duct an } \\
\text { '53" }\end{array}$ & $\begin{array}{l}\text { LEEL: } \\
\text { Sewer }\end{array}$ & $\begin{array}{l}26 \mathrm{fe} \\
\text { Autho }\end{array}$ & ty & & \\
\hline YEAR & JAN & FEB & MAR & APR & MAY & JUN & JUL & AUG & SEP & $\mathrm{OCT}$ & NOV & DEC \\
\hline 1978 & : & & & 13.75 & 13.35 & 15.38 & 15.10 & 16.20 & 16.08 & 15.40 & 12.01 & 14.46 \\
\hline 1979 & $: \quad 15.75$ & 14.41 & 17.42 & 17.97 & 18.29 & 12.83 & 11.65 & 13.82 & 15.36 & 12.69 & 13.25 & 13.40 \\
\hline 1980 & $: 14.82$ & 16.18 & 16.46 & 17.48 & 17.04 & 16.02 & 17.60 & 18.43 & 19.32 & 19.06 & 17.28 & 17.24 \\
\hline 1981 & $: 17.25$ & 14.82 & 13.93 & 16.17 & 15.84 & 10.92 & 10.19 & 10.77 & 12.72 & 13.00 & 12.87 & 12.50 \\
\hline $\begin{array}{l}1982 \\
1983\end{array}$ & $\begin{array}{l}: 10.94 \\
: 11.05\end{array}$ & $\begin{array}{l}12.04 \\
11.95\end{array}$ & $\begin{array}{l}11.98 \\
12.75\end{array}$ & $\begin{array}{l}12.23 \\
12.65\end{array}$ & $\begin{array}{l}12.19 \\
11.48\end{array}$ & 12.28 & 12.24 & 11.69 & 11.40 & $11.70^{\circ}$ & 12.17 & 10.68 \\
\hline
\end{tabular}

a puaping, b recently punped, e estimated, $h$ tape measurement, $j$ lowest water level recorded, n nearby punping well 


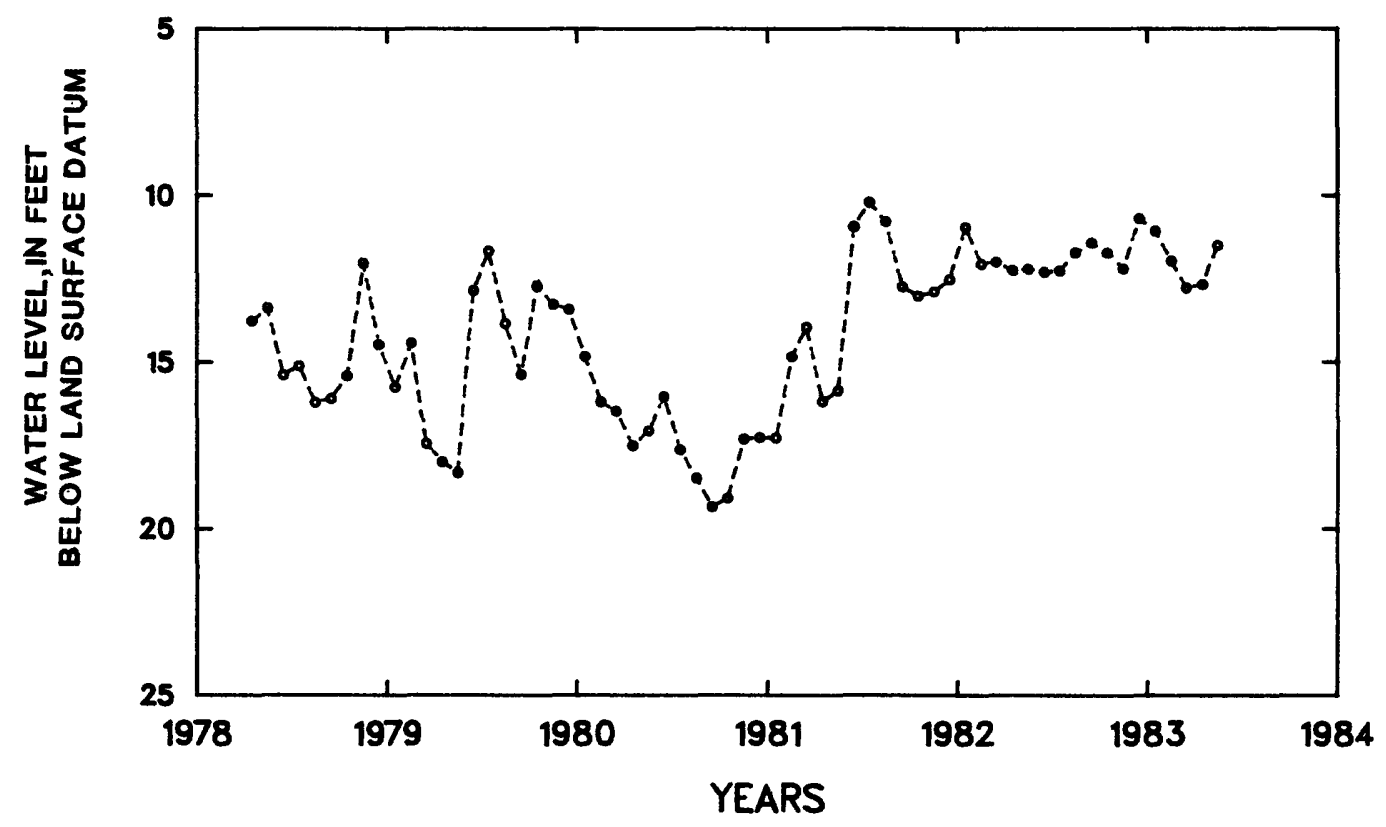

Figure 34.--Ground-water levels at Yabucoa Number 10 or TW5. local number 95. 


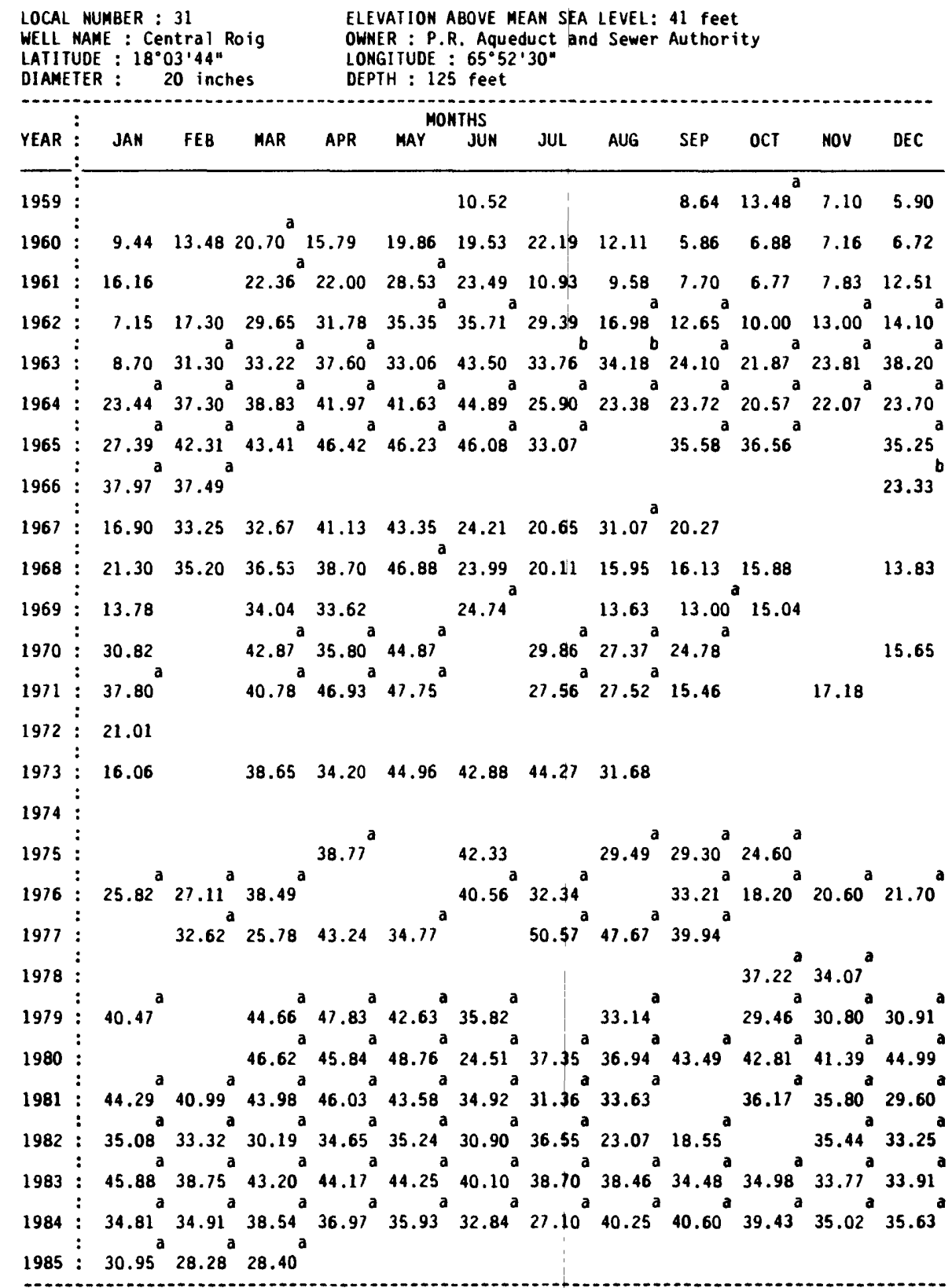

REMARKS: Well discont inued, April 1985.

a pumping, b recently pumped, e estimated, $h$ tape measurement, $j$ lowest water level recorded, n nearby puaping well 


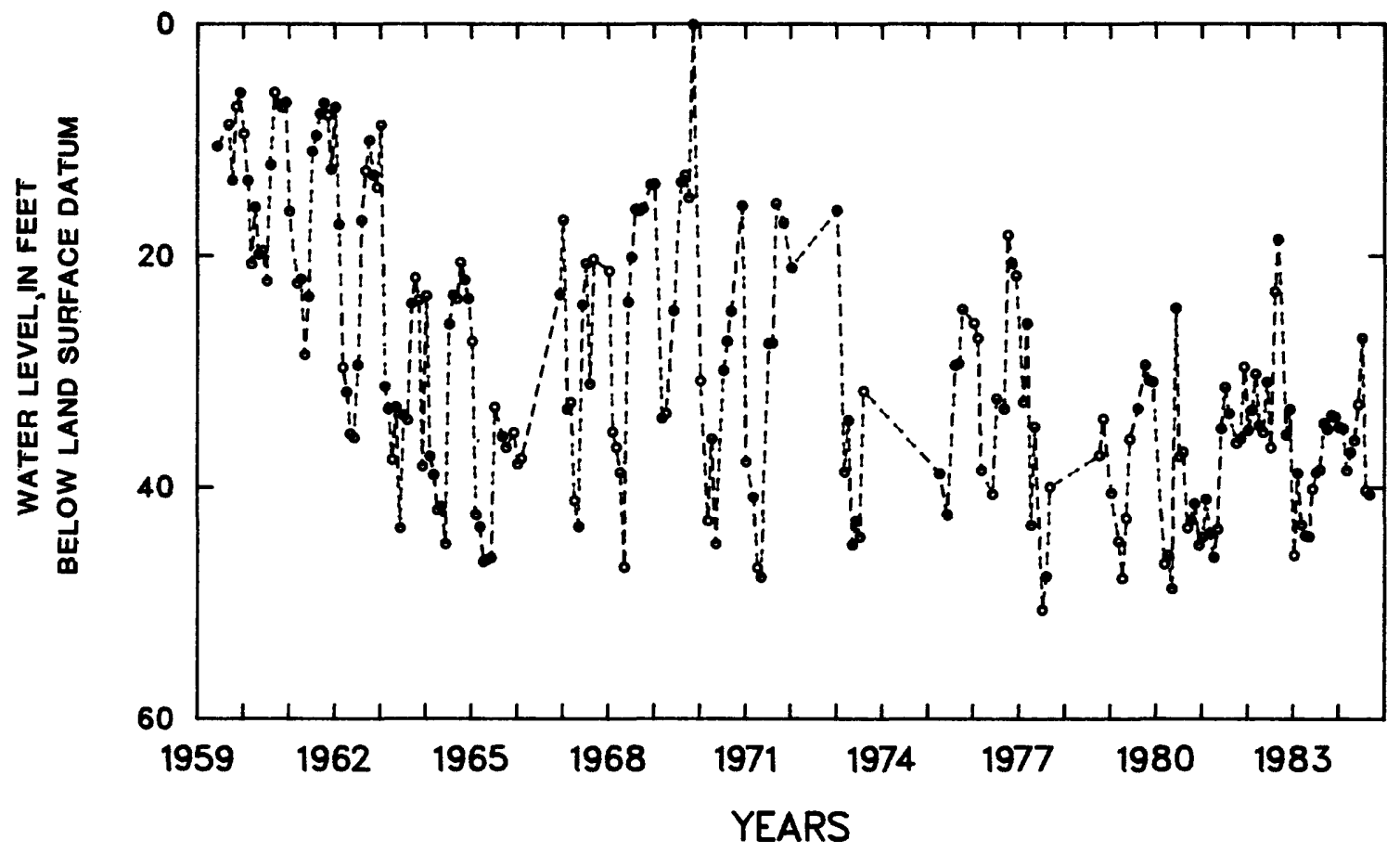

Figure 35.--Ground-water levels at Central Rolg, local number 31 . 


\section{WELLS IN THE \\ EAST COAST PROVINCE \\ Maunabo}

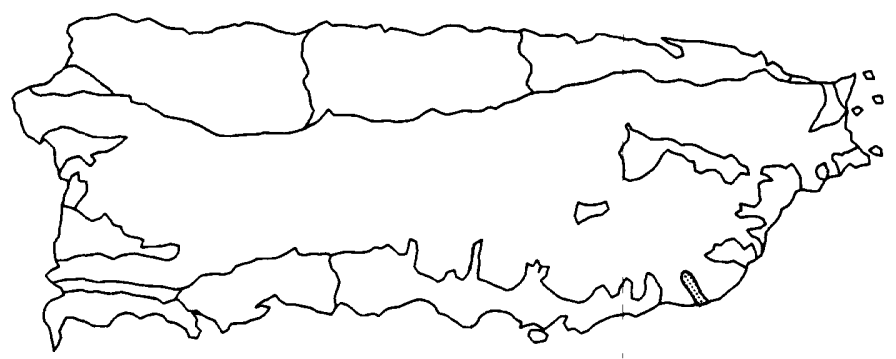

\begin{tabular}{|c|c|c|c|c|c|c|c|c|c|c|c|c|c|}
\hline \multirow{3}{*}{$\begin{array}{l}\text { LOCAL } \\
\text { WELL } \\
\text { LATIT } \\
\text { DIANE } \\
\text { YEAR }\end{array}$} & \multicolumn{4}{|c|}{$\begin{array}{l}\text { NUMBER : } 122 \\
\text { NAME : Maunabo Calzada } \\
\text { TUDE : } 18^{\circ} 00^{\prime} 26^{\prime \prime} \\
\text { ETER : } 4 \text { inches }\end{array}$} & $\begin{array}{l}\text { ELE } \\
\text { OWN } \\
\text { LON } \\
\text { DEP }\end{array}$ & $\begin{array}{l}\text { VATION } \\
\text { ER : P. } \\
\text { GITUDE } \\
\text { TH : } 7\end{array}$ & $\begin{array}{l}\text { BOVE M } \\
\text { Aque } \\
66^{\circ} 54 \\
\text { feet }\end{array}$ & $\begin{array}{l}\text { EAN SEA } \\
\text { Juct an } \\
43^{4}\end{array}$ & $\begin{array}{l}\text { LEVEL: } \\
\text { d Sewer }\end{array}$ & $\begin{array}{l}28.5 \mathrm{f} \\
\text { Author }\end{array}$ & & & \\
\hline & : & & & & & MO & THS & & & & & & \\
\hline & : & JAN & FEB & MAR & APR & MAY & JUN & JUL & AUG & SEP & $\mathrm{OCT}$ & NOV & DEC \\
\hline 971 & $\vdots$ & & & & & & & & & & & & 4.71 \\
\hline 972 & $\vdots$ & 4.92 & 5.27 & 5.56 & 8.63 & 5.74 & 5.76 & 5.26 & 6.49 & 4.97 & 4.27 & 4.62 & 4.38 \\
\hline 1973 & $\vdots$ & & & & & & & & 4.07 & & & & 5.65 \\
\hline 1974 & : & & & & & & & & & 6.29 & & & \\
\hline 975 & $\vdots$ & 4.33 & & 5.08 & 10.56 & & 8.94 & & 6.84 & & 3.57 & & \\
\hline 1976 & $\vdots$ & 3.50 & 4.29 & 3.27 & 3.60 & 4.11 & 4.34 & 2.24 & 5.43 & 4.40 & 6.06 & 5.52 & 6.35 \\
\hline 1977 & $\vdots$ & 5.77 & 10.40 & 11.08 & 9.24 & 11.56 & & & 12.38 & 11.23 & & & \\
\hline 1978 & $\vdots$ & & 9.18 & 9.80 & 8.83 & & 8.70 & 5.46 & 3.50 & 4.09 & 6.66 & 6.19 & \\
\hline 1979 & $\vdots$ & 4.67 & 5.45 & 5.71 & 6.03 & & 6.55 & & 7.29 & & 4.90 & 5.99 & 5.75 \\
\hline 1980 & $:$ & & & 9.39 & 6.39 & 5.80 & 8.02 & 9.84 & 6.79 & 9.87 & 7.64 & 9.39 & 10.05 \\
\hline 1981 & $\vdots$ & 7.69 & 6.21 & 10.46 & 11.13 & 10.85 & 9.16 & 8.77 & 8.70 & 8.20 & 8.40 & 8.65 & 5.90 \\
\hline 1982 & : & 8.08 & 8.40 & 9.01 & 9.02 & 8.37 & 8.38 & 9.47 & 10.50 & 9.50 & & $1.02^{\prime \prime}$ & $9.17^{11}$ \\
\hline & : & & 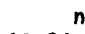 & $n$ & $n$ & $n$ & $n$ & $n$ & $n$ & $n$ & $n$ & $n$ & \\
\hline 1983 & : & 9.09 & 10.04 & 10.90 & 11.55 & 11.22 & 7.94 & 8.19 & 8.72 & 8.32 & 8.90 & 8.78 & 9.42 \\
\hline & : & & & $n$ & $n$ & n & & & 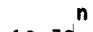 & & & 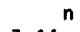 & \\
\hline 1984 & : & 9.14 & 10.10 & 10.10 & 10.60 & 11.33 & 8.13 & 10.45 & 10.79 & 7.83 & 6.10 & 7.11 & 7.69 \\
\hline 985 & : & $8.91^{n}$ & $9.73^{n}$ & $9.32^{n}$ & & & & & & & & & \\
\hline
\end{tabular}

REMARKS: Well discontinued, April 1985.

a pumping. b recently pumped, e estimated, $h$ tape measurement, $j$ lowest water level recorded,

n nearby puaping well 


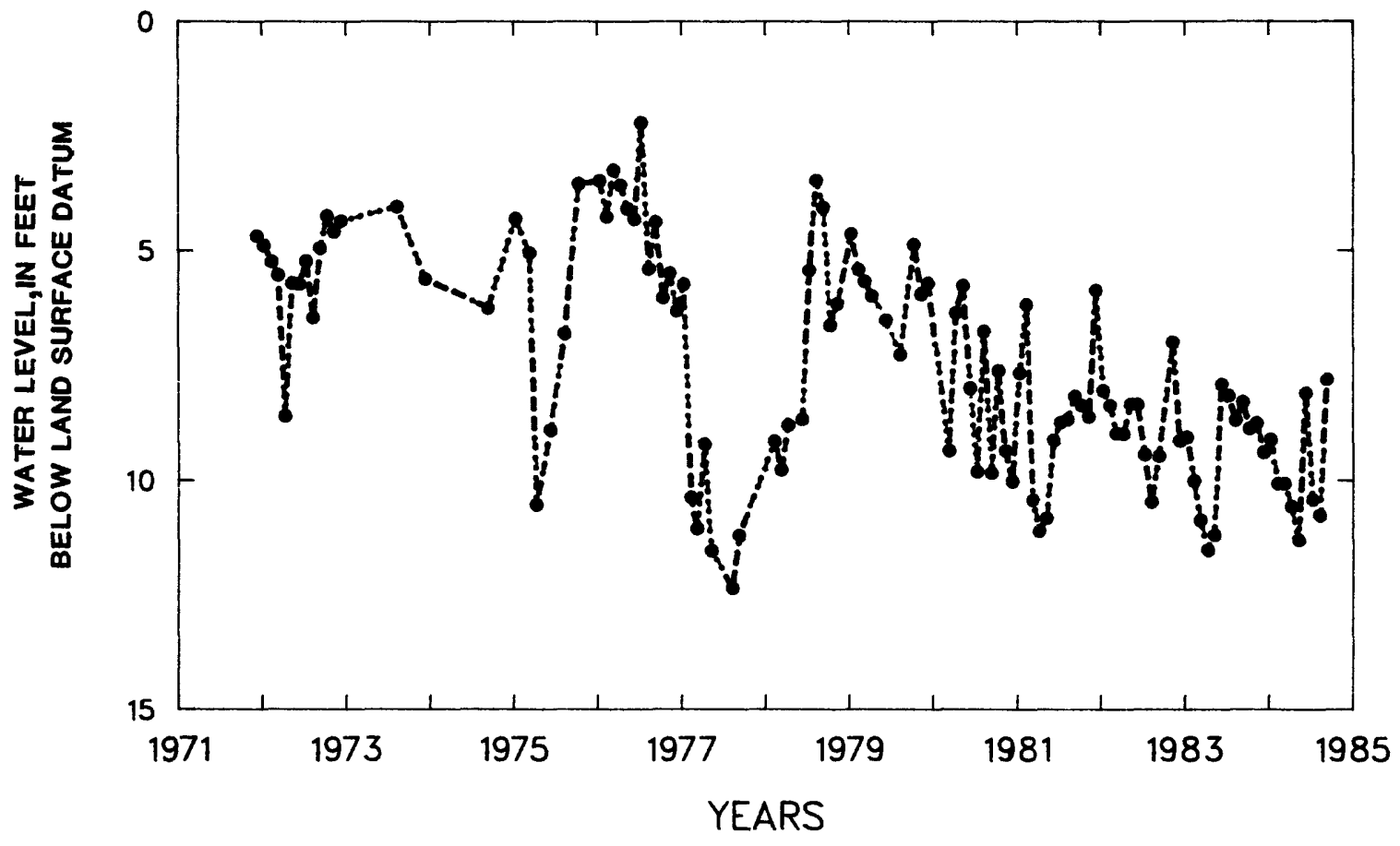

Flgure 36.--Ground-water levels at Maunabo Caizada, local number 122. 


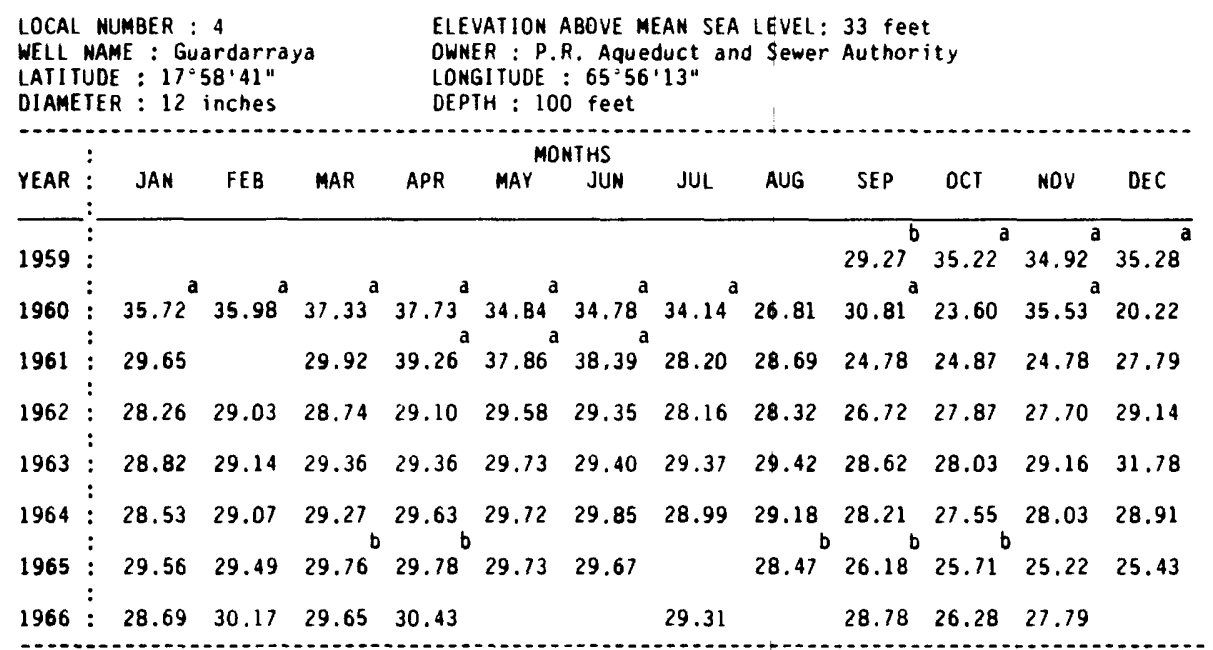

REMARKS: Well discontinued, December, 1966.

a pumping, b recently pumped, e estiwated, $h$ tape measurenent, $j$ lowest water level recorded, n nearby pumping well 
WELLS IN THE

LAJAS VALLEY

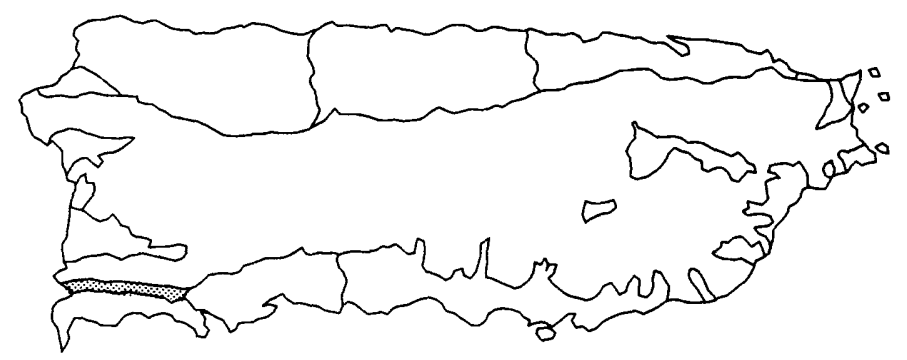

LOCAL NUMBER : 143

ELEVATION ABOVE MEAN SEA LEVEL: 52.5 feet

WELL NAME : Vivonl, Hac.Amistad OWNER : Pedro P. Vivoni

LATITUDE : $18^{\circ} 01^{\prime} 32^{\prime \prime}$

DIAMETER : 12 inches

LONGITUDE : 67 $67^{\circ} 38^{\prime \prime}$

DEPTH : 200 feet

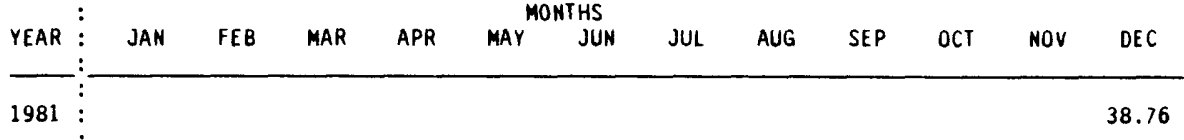

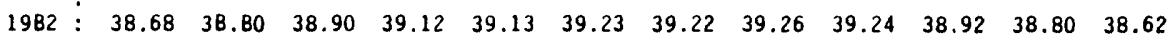

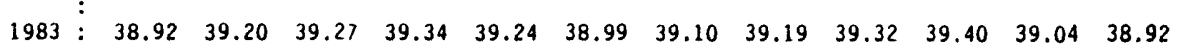

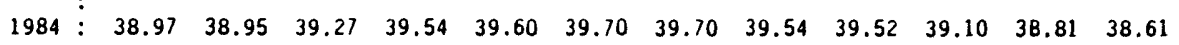

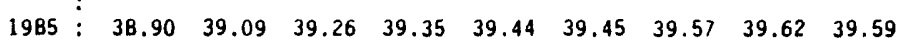

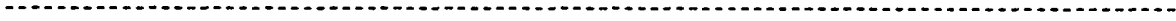

a pumping, b recently pumped, e estimated, $h$ tape measurement, $j$ lowest water level recorded, n nearby pumping well

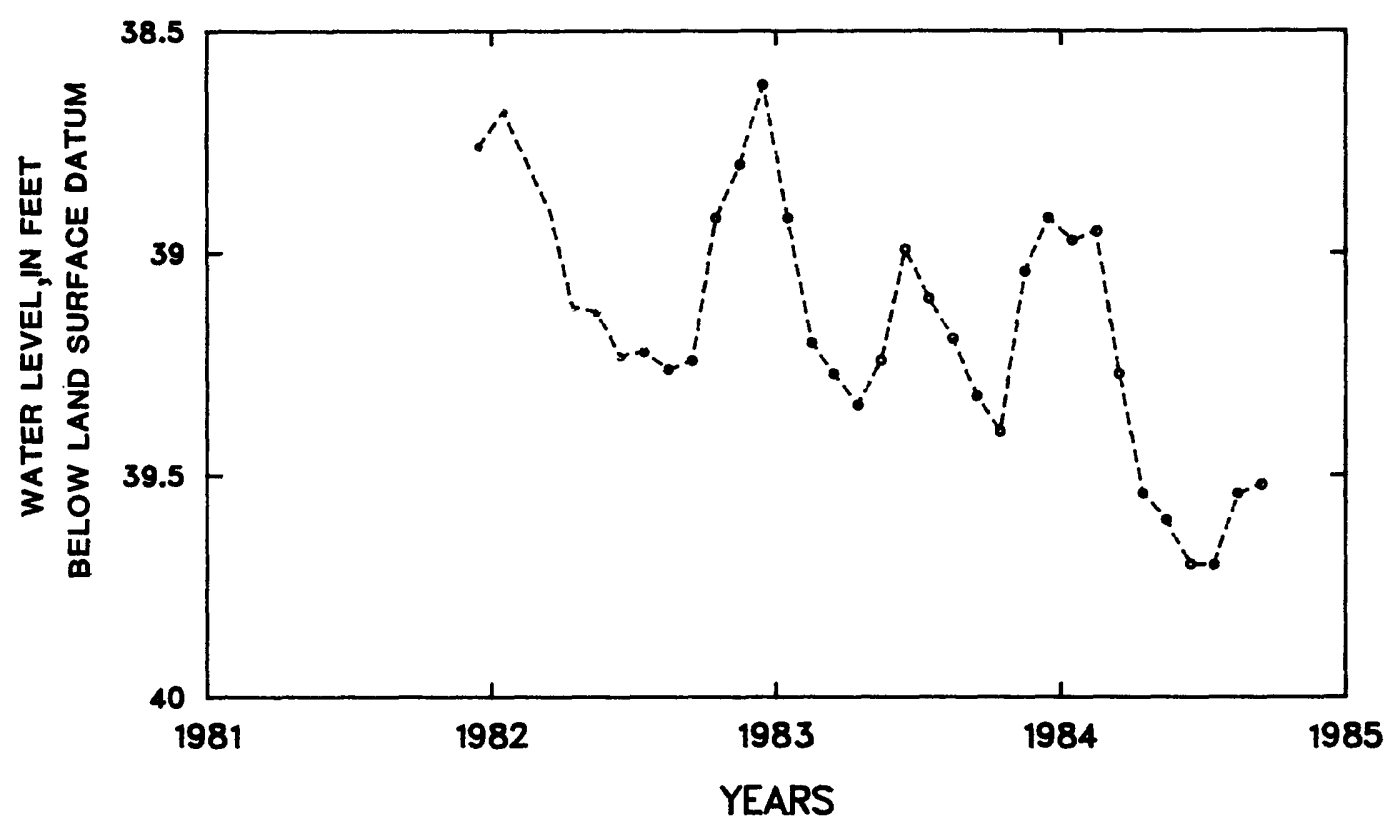

Figure 37.--Ground-water levels at Vivoni, Hacienda Amistad, local number 143. 


\section{WELLS IN THE INTERIOR PROVINCE}

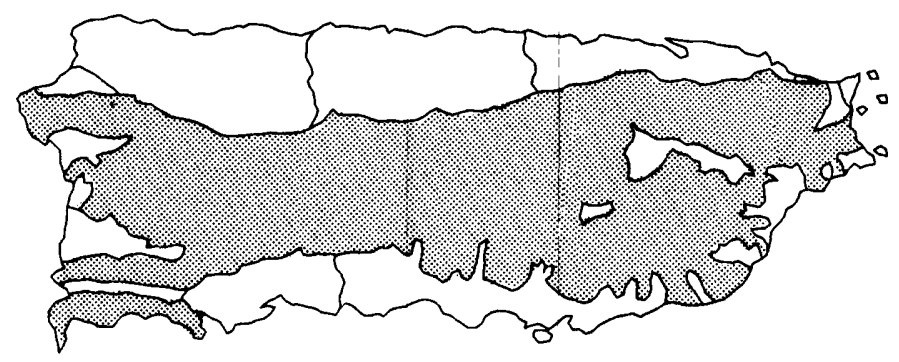

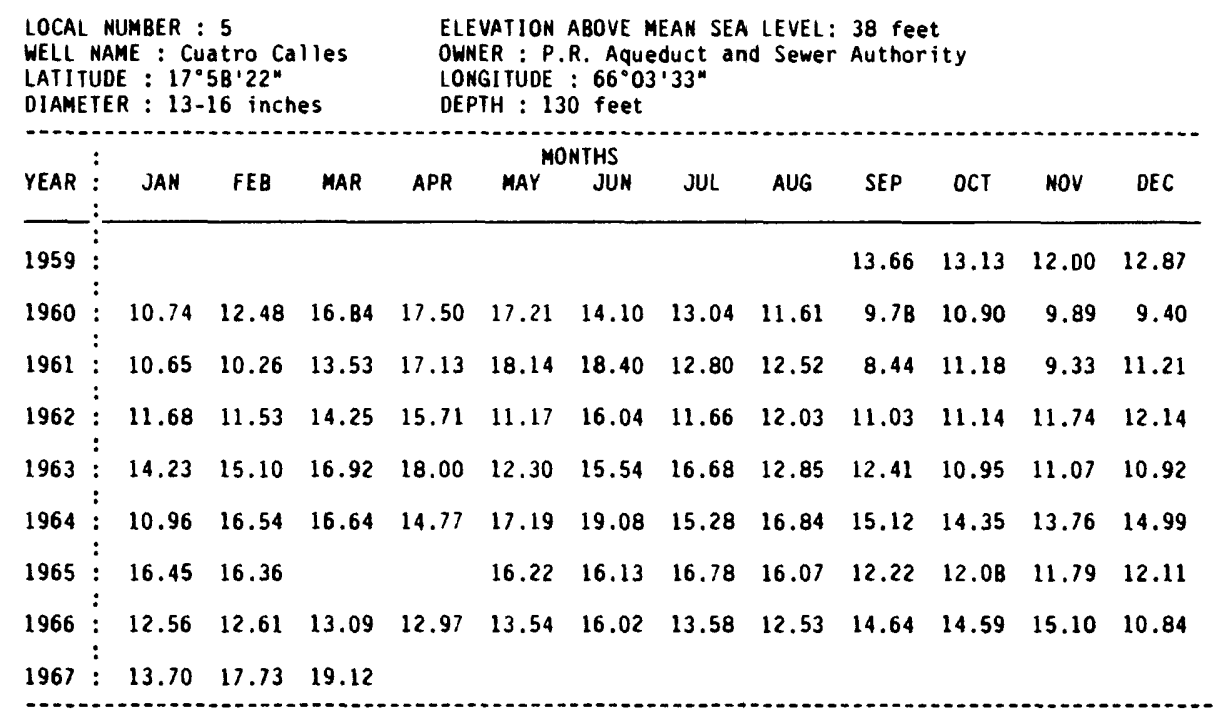

REMARKS: Well destroyed, April 1967.

a pumping, b recently pumped, e estimated, $h$ tape measurement, $j$ lowest water level recorded, n nearby pumping well 


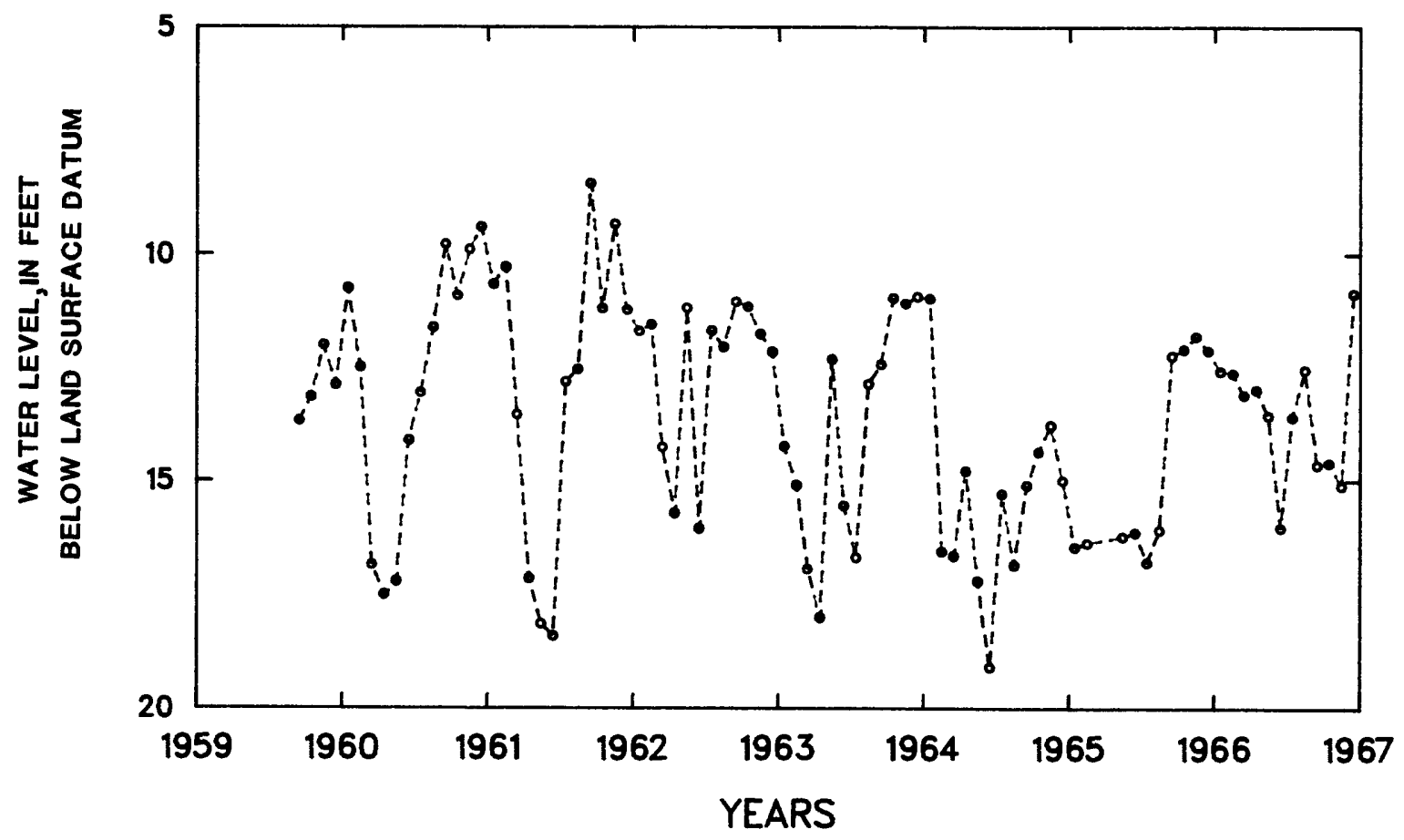

Figure 38.--Ground-water levels at Cuatro Calles, local number 5 . 


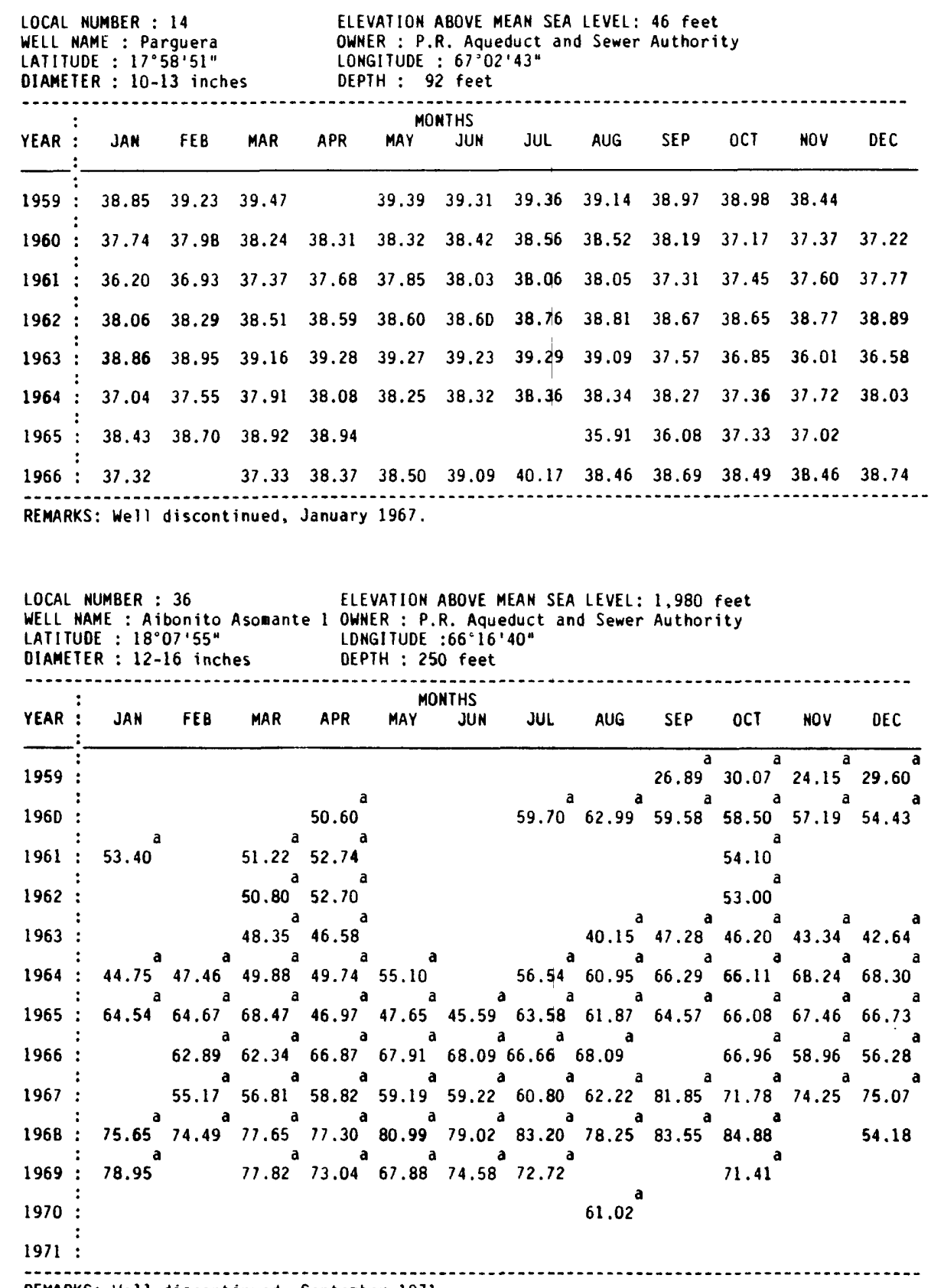

REMARKS: Well discont inued, September 1971.

a pumping, b recently pumped, e estimated, $h$ tape measurement, $j$ lowest water level recorded, n nearby pumping well 


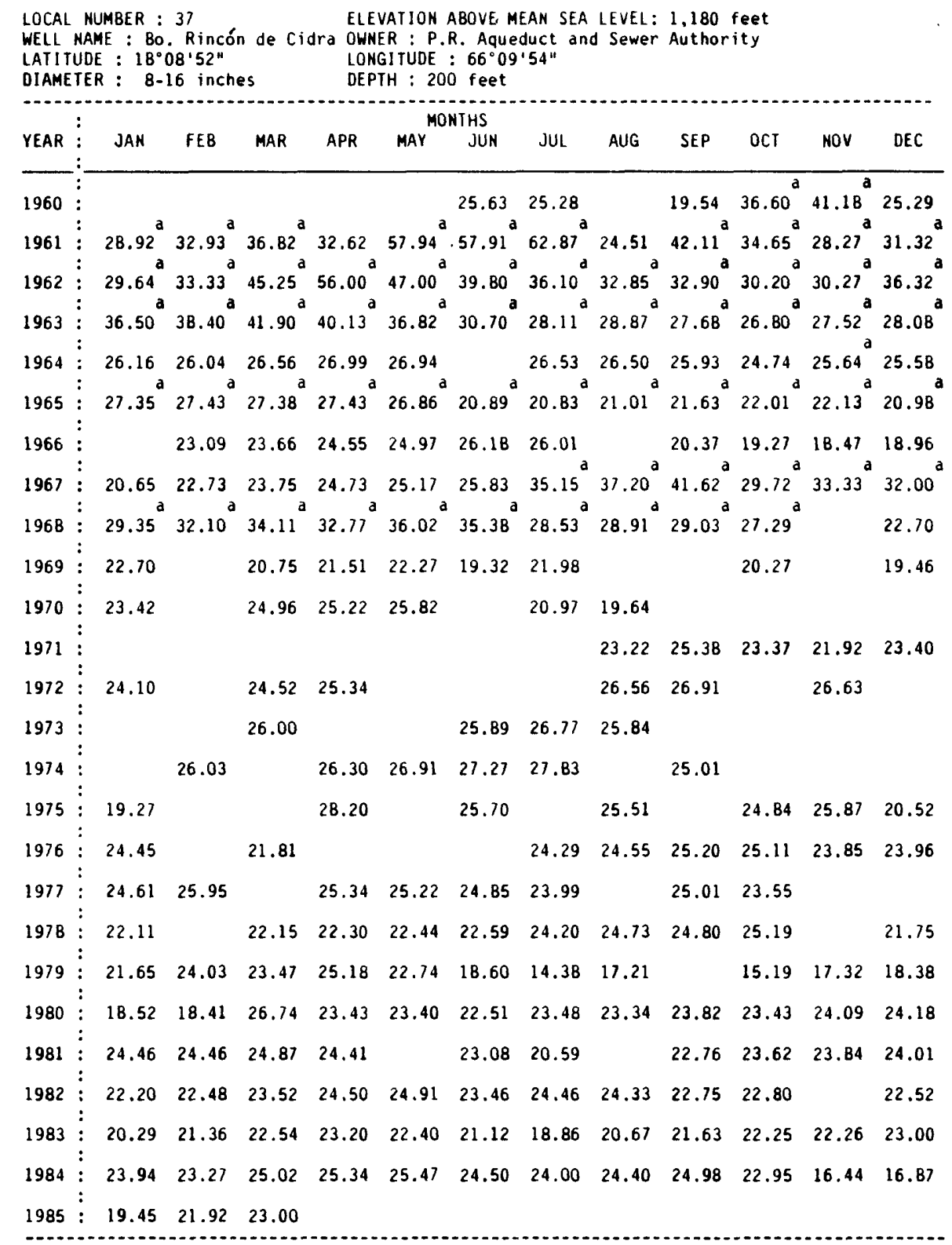

a punping, b recently pumped, e estimated, $h$ tape measurement, $j$ lowest water level recorded, n nearby pumping well 


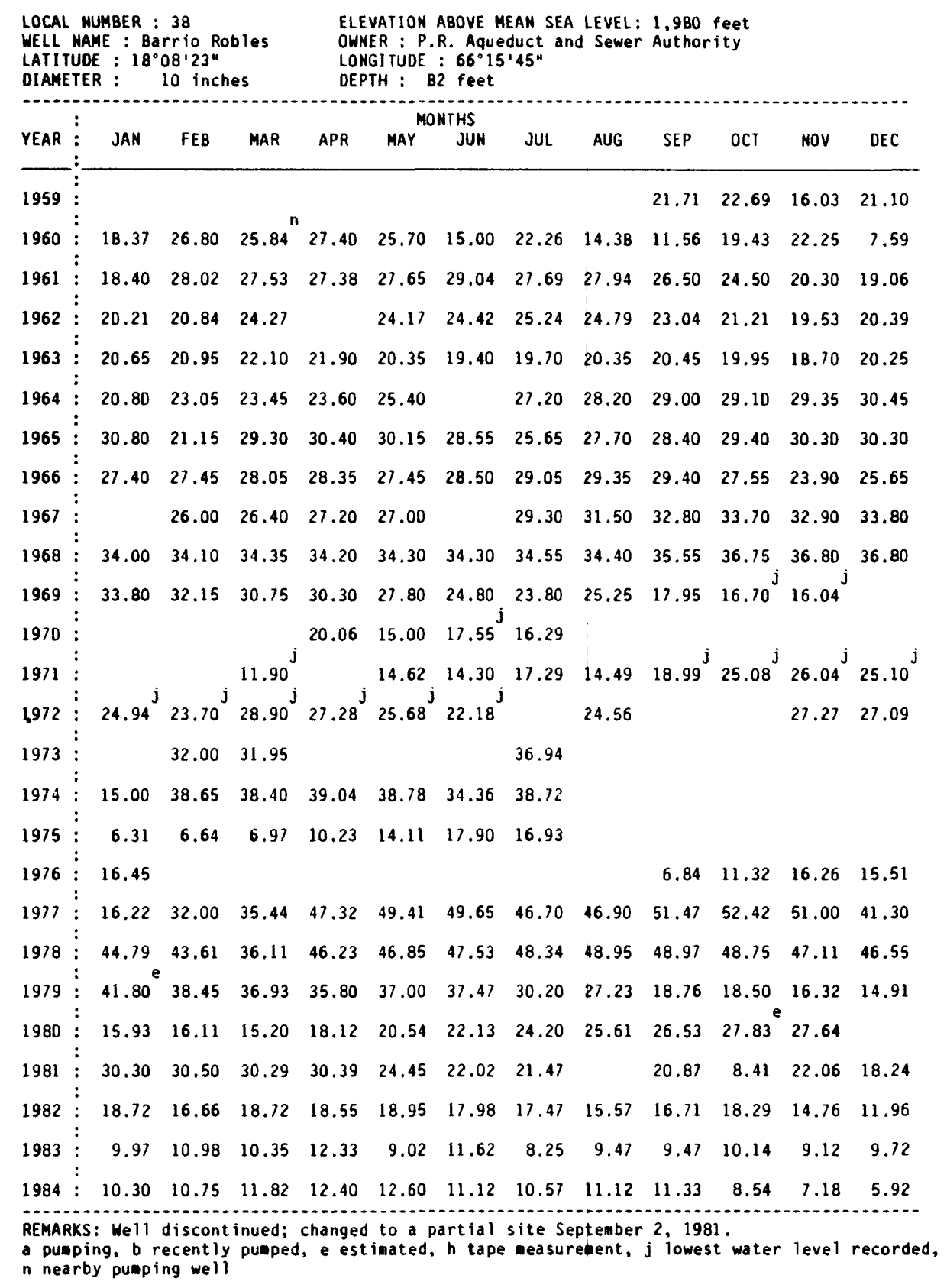




\begin{tabular}{|c|c|c|c|c|c|c|c|c|c|c|c|c|}
\hline $\begin{array}{l}\text { LOCAL } \\
\text { WELL } \\
\text { LATITL } \\
\text { DIAME }\end{array}$ & $\begin{array}{l}\text { NUMBER : } \\
\text { NAME : Pr } \\
\text { UDE : } 18^{\circ} \\
\text { TER : }\end{array}$ & $\begin{array}{l}39 \\
\text { oyecto } \\
09^{\prime} 17 " 1 \\
10 \text { inch }\end{array}$ & $\begin{array}{l}\text { a Plat } \\
\text { es }\end{array}$ & $\begin{array}{l}\text { ELEY } \\
\text { OWNE } \\
\text { LONC } \\
\text { DEPI }\end{array}$ & $\begin{array}{l}\text { VATION } \\
\text { ER : P. } \\
\text { GITUDE } \\
\text { TH : } 12\end{array}$ & $\begin{array}{l}\text { ABOVE M } \\
\text { R. Aque } \\
: 66^{\circ} 13 \\
0 \text { feet }\end{array}$ & $\begin{array}{l}\text { EAN SE } \\
\text { duct a } \\
\text { '56" }\end{array}$ & IEVEL: & $\begin{array}{l}951 \mathrm{fe} \\
\text { Author }\end{array}$ & $y$ & & \\
\hline YEAR & JAN & FEB & MAR & APR & MAY & $\begin{array}{l}\text { NTHS } \\
\text { JUN }\end{array}$ & JUL & AUG & SEP & OCT & NOV & DEC \\
\hline 1959 & $\vdots$ & & & & & & & & $64.50^{a}$ & $71.45^{\mathrm{a}}$ & $72.10^{a}$ & $72.15^{\mathrm{a}}$ \\
\hline 1960 & $\vdots \quad 72.20^{\mathrm{a}}$ & $57.29^{\mathrm{a}}$ & $71.85^{\circ}$ & $72.50^{\mathrm{a}}$ & $73.80^{\mathrm{a}}$ & $72.20^{\circ}$ & $72.20^{a}$ & $72.90^{\circ}$ & $72.90^{a}$ & $71.70^{\mathrm{a}}$ & $57.52^{a}$ & $68.90^{a}$ \\
\hline 1961 & $: 70.80^{\mathrm{a}}$ & 51.73 & $72.19^{\mathrm{a}}$ & $71.70^{\mathrm{a}}$ & 54.23 & $56.97^{\mathrm{a}}$ & $71.72^{\mathrm{a}}$ & $71.75^{\mathrm{a}}$ & $70.97^{a}$ & $79.50^{\mathrm{a}}$ & $68.84^{a}$ & $67.84^{\mathrm{a}}$ \\
\hline 1962 & $: \quad 65.39^{\mathrm{a}}$ & $63.60^{\mathrm{a}}$ & $59.90^{\mathrm{a}}$ & $60.85^{\mathrm{a}}$ & $69.00^{\mathrm{a}}$ & $79.10^{\mathrm{a}}$ & $79.00^{a}$ & $78.00^{a}$ & $79.00^{\mathrm{a}}$ & $72.00^{\mathrm{a}}$ & & \\
\hline 1963 & $\dot{*}$ & & & & & & & & $70.09^{\mathrm{d}}$ & & & $70.73^{\mathrm{a}}$ \\
\hline 1964 & $: \quad 69.06^{\mathrm{a}}$ & $67.10^{\mathrm{a}}$ & $72.89^{\mathrm{a}}$ & $71.59^{\mathrm{a}}$ & 57.10 & & $69.75^{\mathrm{a}}$ & $6 B .15^{\circ}$ & $69.79^{a}$ & $69.34^{\mathrm{a}}$ & $68.77^{a}$ & $68.85^{\mathrm{a}}$ \\
\hline 1965 & $: 70.82^{\mathrm{a}}$ & $70.9 \mathrm{~B}^{\mathrm{a}}$ & $75.33^{\mathrm{a}}$ & $B 0.06^{\mathrm{d}}$ & $91.68^{a}$ & $72.96^{\mathrm{a}}$ & $74.32^{\mathrm{a}}$ & $72.67^{a}$ & $85.49^{a}$ & $86.77^{\mathrm{a}}$ & $87.13^{a}$ & $86.76^{\mathrm{a}}$ \\
\hline 1966 & : & & & & & & & & & & & \\
\hline 1967 & : & & & & 49.47 & & & & & & & \\
\hline
\end{tabular}

REMARKS: Well discont inued, June 1967.

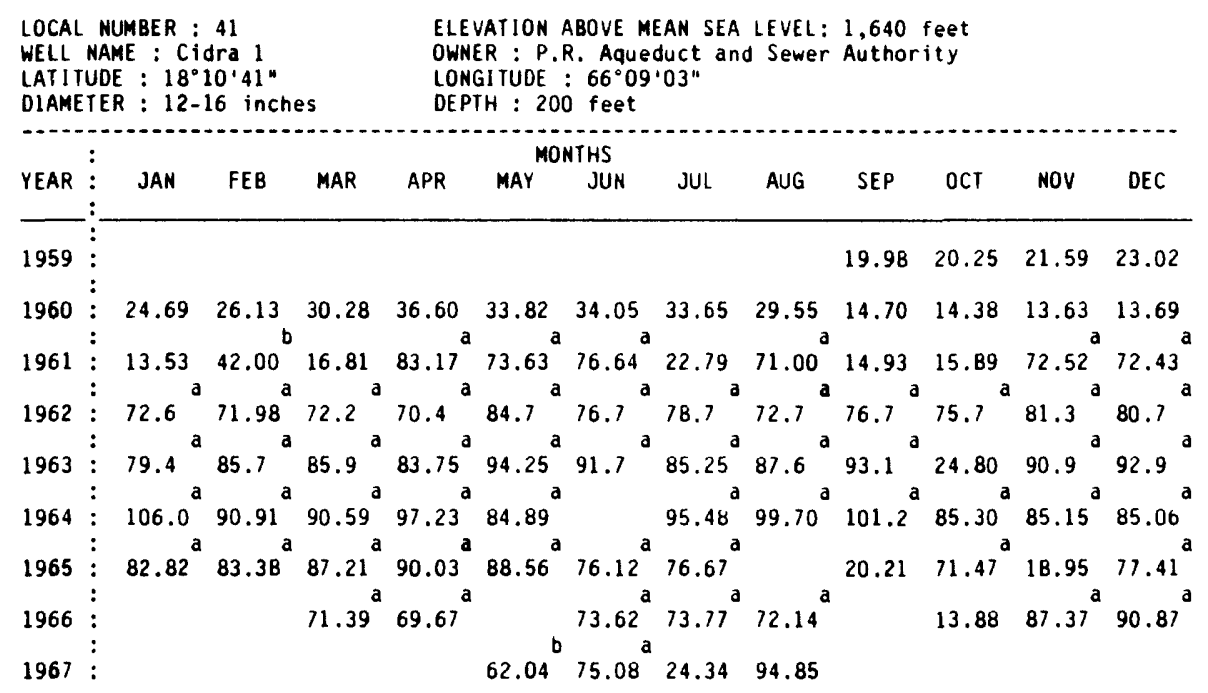

REMARKS: Well discont inued, September 1967.

a pumping, b recently pumped, e estimated, $h$ tape measurement, $j$ lowest water level recorded, n nearby pumping well 


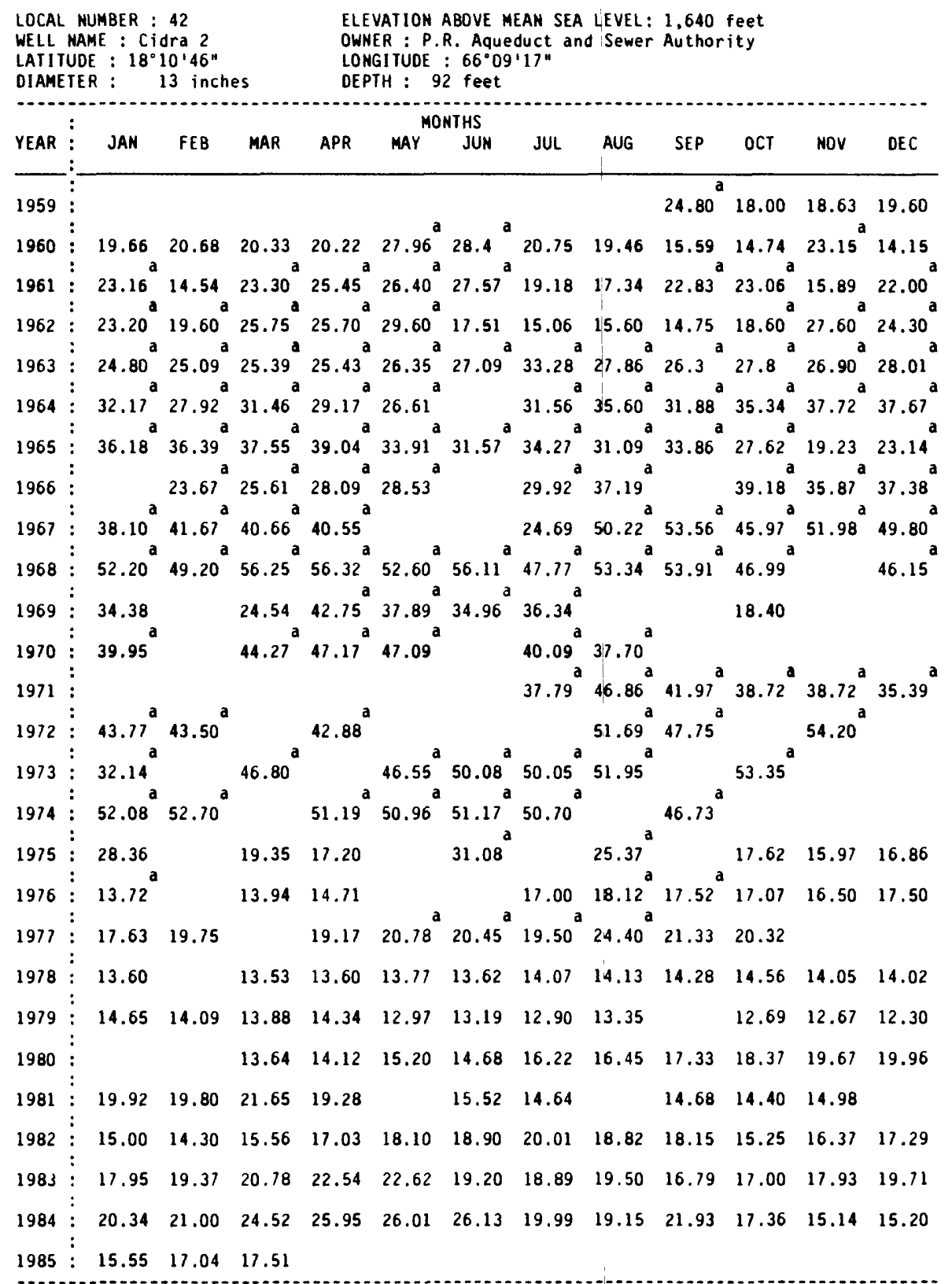

REMARKS: Well discontinued, April 1985.

a pumping, b recently punped, e estimated, $h$ tape measurement, $j$ lowest water level recorded, n nearby pumping well 
THIS PAGE WAS LEFT BLANK INTENTIONALLY 


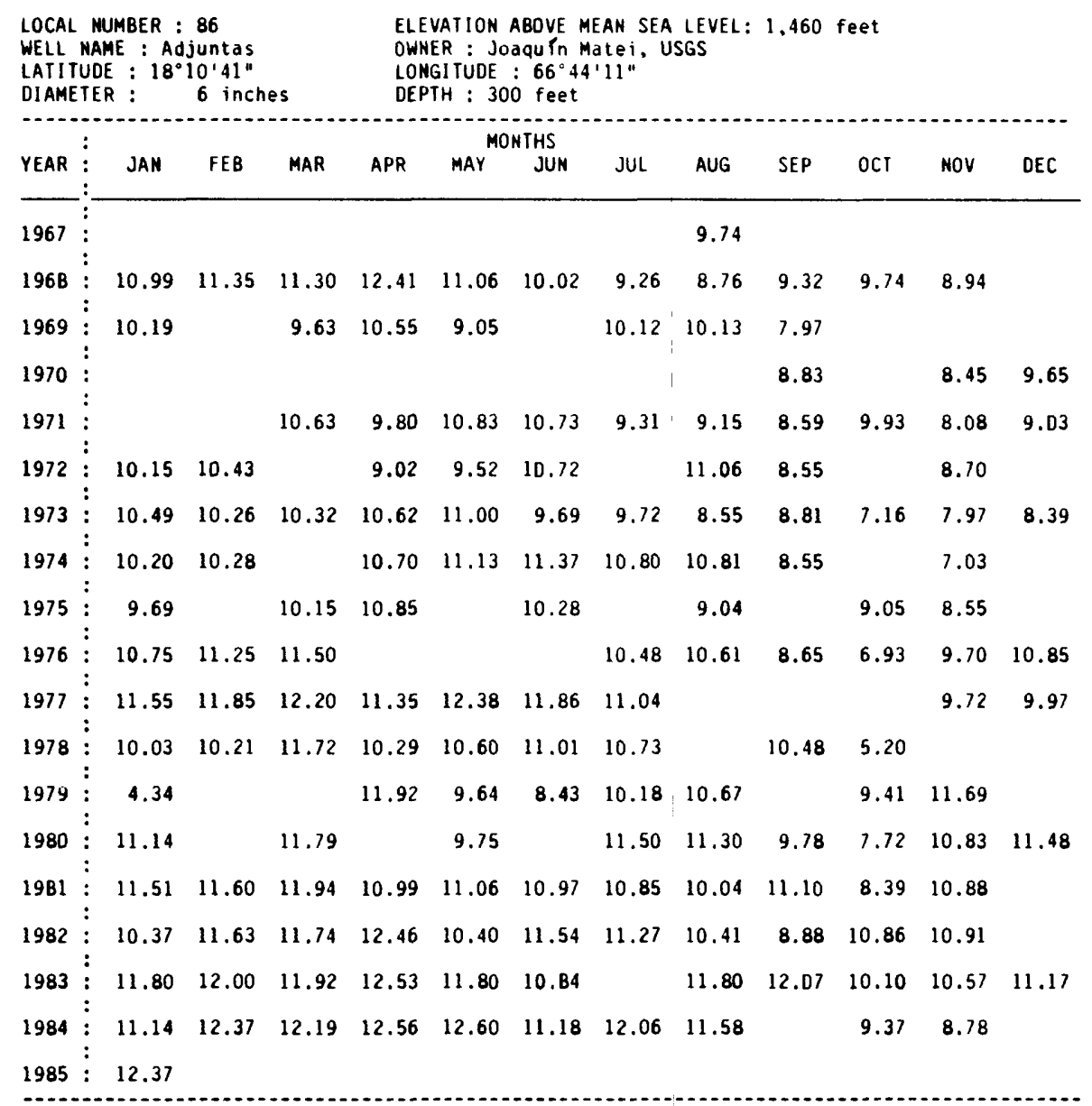

REMARKS: Well discontinued, February 1985.

a pumping, b recently pumped, e estimated, $h$ tape measurement, $j$ lowest water level recorded,

n nearby pumping well 


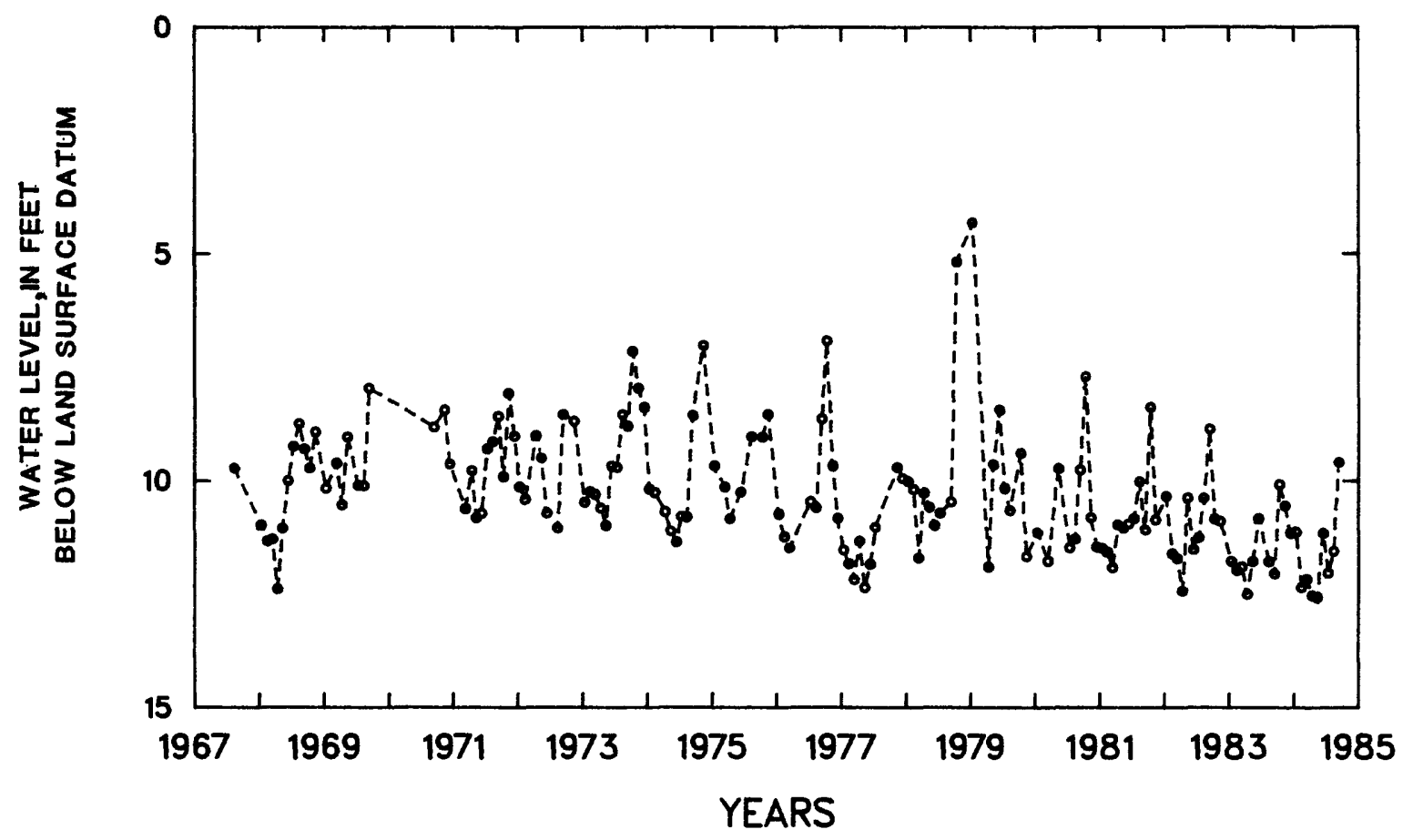

Figure 39.--Ground-water levels at Adjuntas, local number 86. 


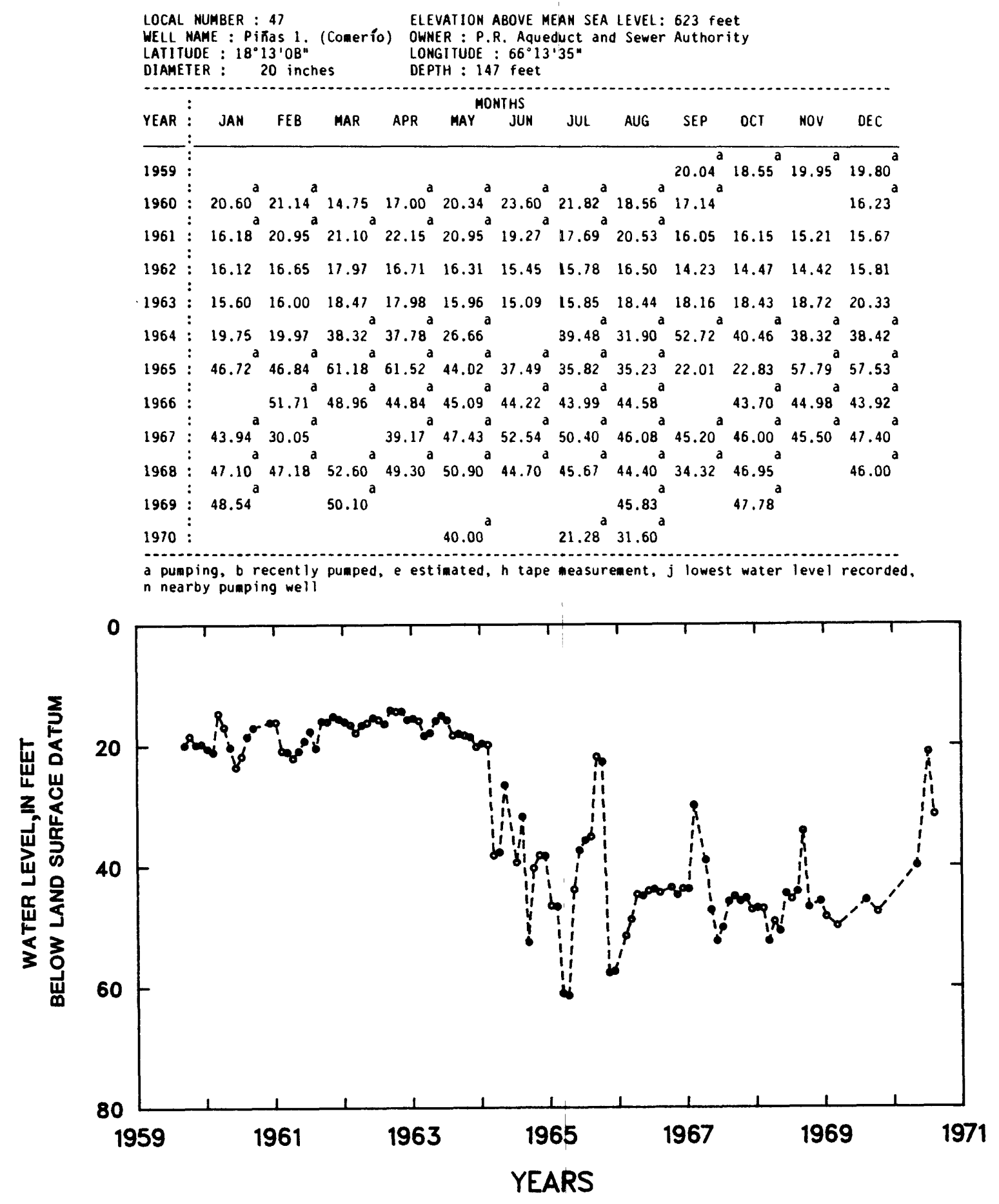

Figure 40.--Ground-water levels at Pinas 1. (Comerio), local number 47. 


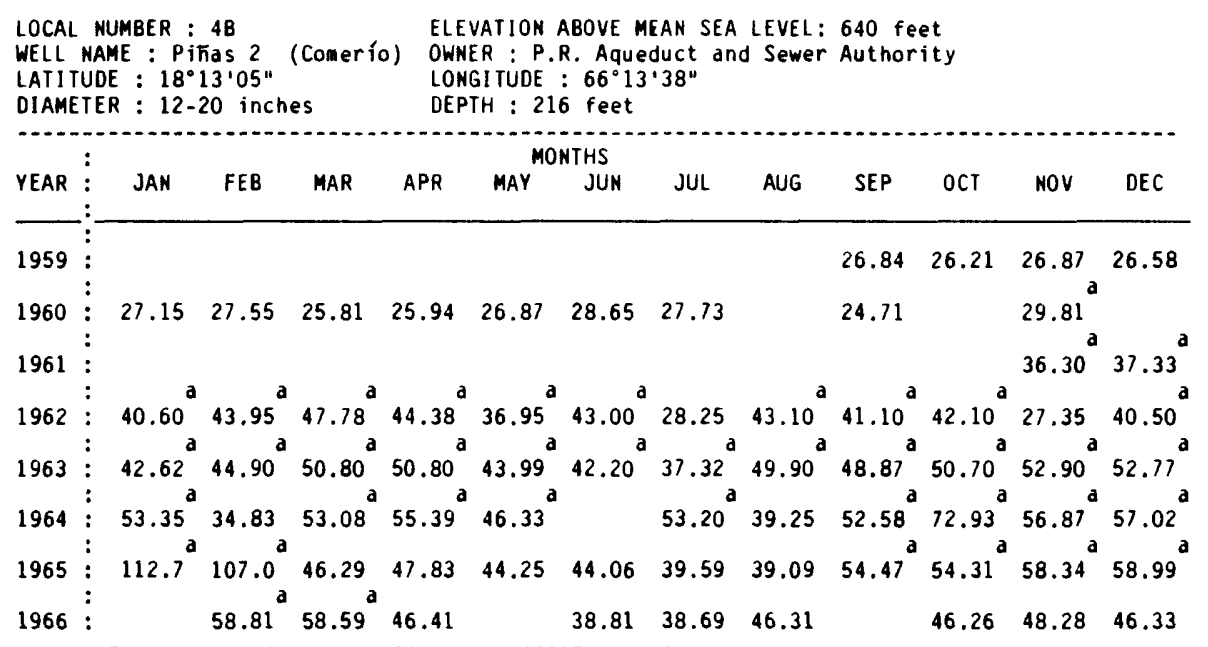

REMARKS: Well discont inued, December 1966.

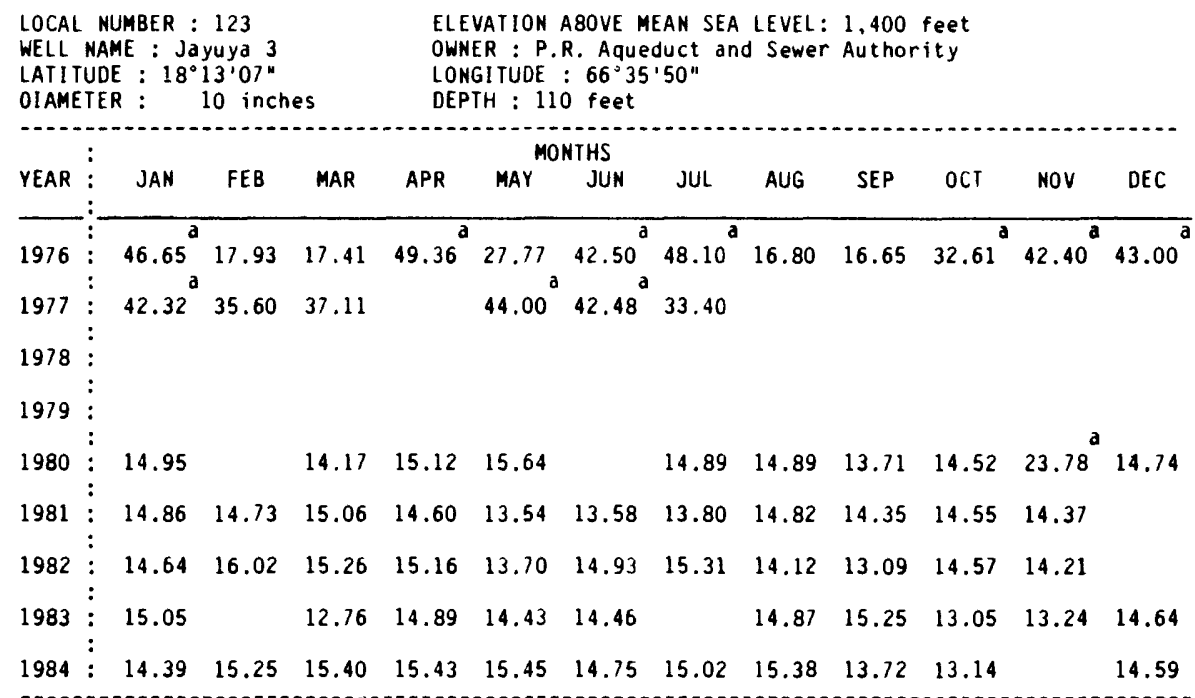

REMARKS: Well discontinued during December 1984.

a pumping, b recently pumped, e estimated, h tape measurement, j lowest water level recorded, n nearby pumping well 


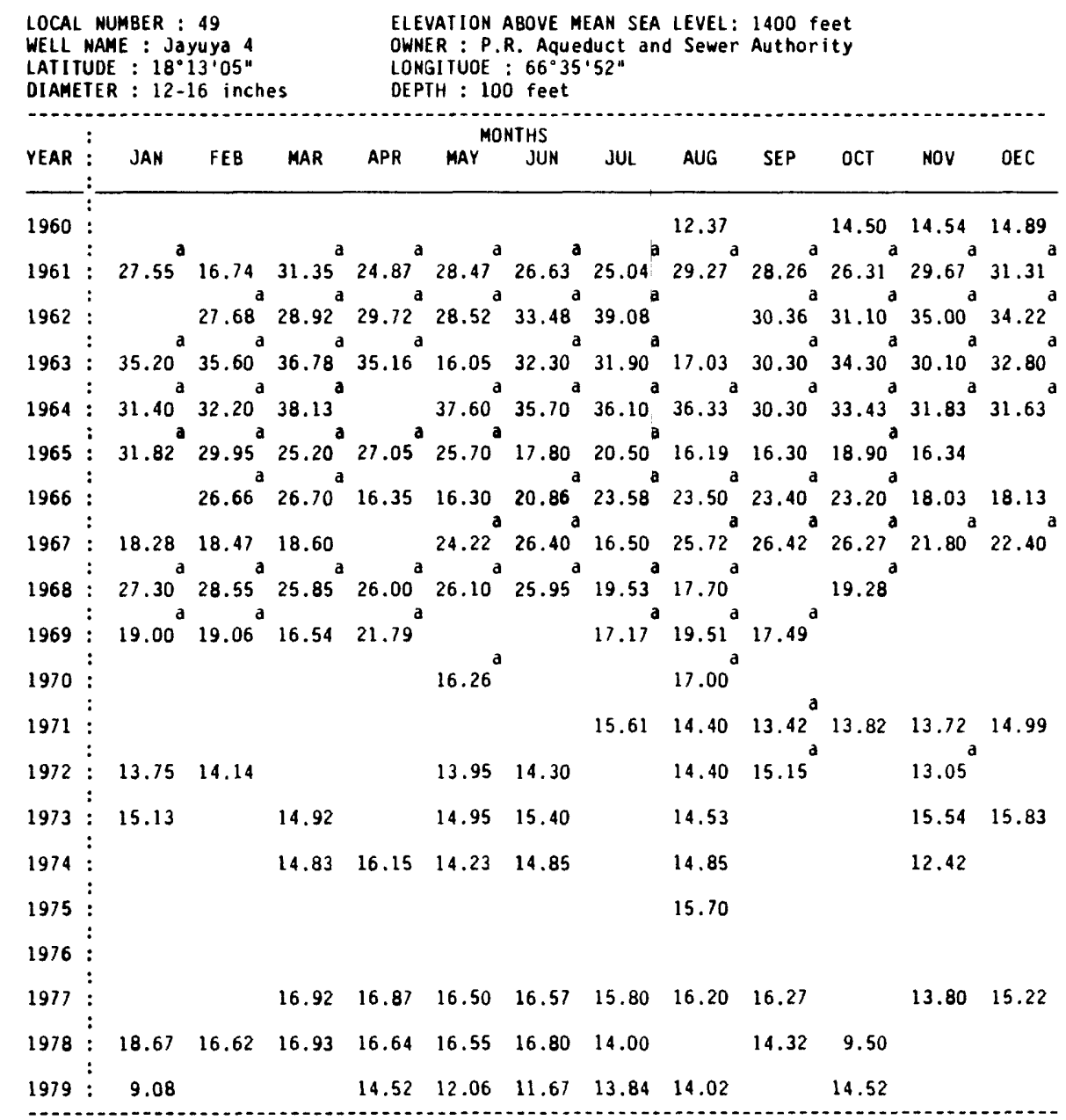

REMARKS: Well discont inued, November 1979

a pumping, b recently pumped, e estimated, $h$ tape measurement, $j$ lowest water level recorded, n nearby pumping well 


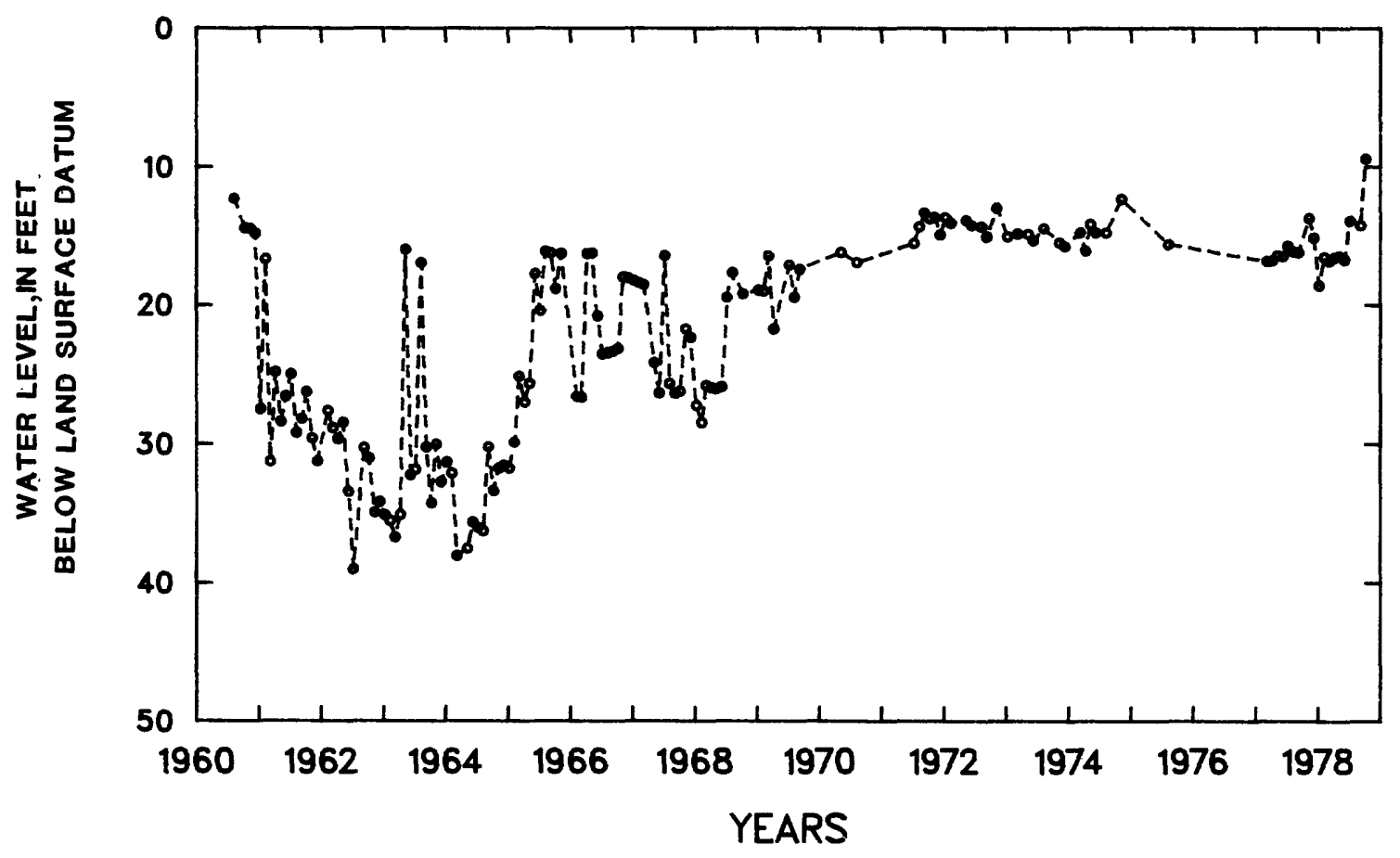

Figure 41.--Ground-water levels at Jayuya 4, local number 49 . 


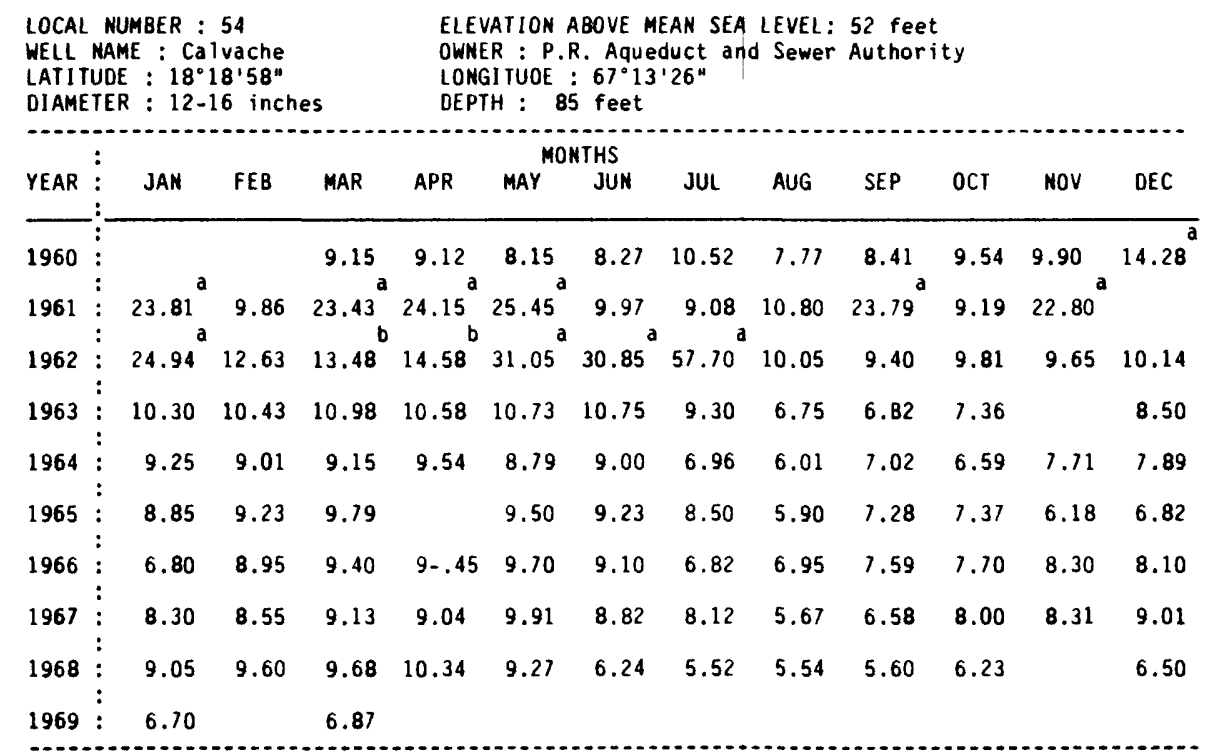

REMARKS: Well discont inued, September 1969.

\begin{tabular}{|c|c|c|c|c|c|c|c|c|c|c|c|c|c|}
\hline $\begin{array}{l}\text { LOCAL } \\
\text { WELL } \\
\text { LATII } \\
\text { OIAME }\end{array}$ & $\begin{array}{l}\text { NU } \\
\text { NAM } \\
\text { TUDE } \\
\text { ETER }\end{array}$ & $\begin{array}{l}\text { UMBER : } \\
\text { ME : Sa } \\
\text { E : } 18^{\circ} \\
\text { R : }\end{array}$ & $\begin{array}{l}83 \\
n \text { Sebas } \\
20^{\prime} 18^{\prime \prime} \\
6 \text { inch }\end{array}$ & $\begin{array}{l}\text { tían } \\
\text { es }\end{array}$ & $\begin{array}{l}\text { ELE } \\
\text { OWN } \\
\text { LON } \\
\text { DEP }\end{array}$ & $\begin{array}{l}\text { VATION } \\
\text { ER : P. } \\
\text { GITUDE } \\
\text { TH : } 30\end{array}$ & $\begin{array}{l}\text { ABOVE } \\
\text { R. Wate } \\
: 66^{\circ} 59 \\
0 \text { feet }\end{array}$ & $\begin{array}{l}\text { AN SEA } \\
\text { ReSOU } \\
32^{\prime \prime}\end{array}$ & $\begin{array}{l}\text { LEVEL: } \\
\text { rces Au }\end{array}$ & $\begin{array}{l}230 \\
\text { thori }\end{array}$ & & & \\
\hline & : & & & & & & NTHS & & & & & & \\
\hline YEAR & : & JAN & FEB & MAR & $A P R$ & MAY & JUN & JUL & AUG & SEP & $\mathrm{OCT}$ & NOV & DEC \\
\hline 1967 & $\vdots$ & & & & & 32.64 & & & & & & & \\
\hline 1968 & $\vdots$ & 31.11 & 30.77 & 30.82 & 31.18 & 30.90 & 27.99 & 28.22 & 28.39 & 28.44 & 28.13 & & 28.13 \\
\hline 1969 & $\vdots$ & 30.09 & & 30.55 & 29.05 & 28.35 & 28.69 & 28.94 & 28.69 & & & 28.88 & \\
\hline 1970 & $\begin{array}{l}: \\
\vdots\end{array}$ & & & & & & & 40.20 & 29.17 & 28.37 & & & \\
\hline 1971 & : & & & & & 28.07 & 28.80 & 28.54 & 28.37 & 28.30 & 28.25 & 28.64 & \\
\hline 1972 & $\vdots$ & & 31.20 & & 30.47 & 28.97 & & & & & 27.41 & & \\
\hline 1973 & : & & 31.19 & 30.80 & & 31.13 & 31.61 & 28.29 & 29.53 & 28.08 & & & 29.69 \\
\hline 1974 & $:$ & & & 30.88 & 31.14 & 31.39 & 28.89 & & 30.11 & 26.34 & & 28.59 & \\
\hline 1975 & : & 30.61 & & 31.01 & 32.17 & & 29.99 & 28.85 & & 28.33 & & & 29.21 \\
\hline 1976 & : & 32.45 & & & 21.95 & 29.88 & 30.46 & 28.58 & 29.20 & 30.99 & 28.40 & 26.89 & 26.35 \\
\hline 1977 & $\vdots$ & 30.40 & 30.66 & 30.96 & 31.18 & 30.52 & 29.32 & 28.80 & 29.32 & 28.35 & 29.69 & & 29.48 \\
\hline 1978 & $\vdots$ & 30.60 & 30.37 & 30.27 & 30.00 & 28.49 & 27.67 & & 28.31 & 28.44 & 28.06 & 29.07 & 30.08 \\
\hline 1979 & $:$ & 30.00 & 29.26 & & & & 26.82 & & & & 27.67 & 29.73 & 28.98 \\
\hline 1980 & : & 29.73 & 30.18 & 30.46 & 29.65 & 28.47 & & 28.77 & 28.64 & 28.26 & 28.20 & 28.70 & 29.46 \\
\hline 1981 & $\vdots$ & 29.88 & 29.63 & 30.02 & 28.69 & 29.35 & 26.83 & 28.35 & 28.69 & 28.80 & 28.60 & 27.42 & 28.18 \\
\hline 1982 & : & 29.05 & 25.64 & 30.05 & 30.20 & 27.83 & 27.24 & 27.22 & 26.47 & 28.31 & 28.67 & 28.74 & 28.90 \\
\hline 1983 & : & 29.04 & 29.75 & 30.22 & 30.71 & 29.38 & 26.88 & 28.53 & 30.55 & 27.96 & 27.90 & 27.85 & 29.08 \\
\hline 1984 & $\vdots$ & 29.72 & 30.12 & 30.18 & 30.44 & 30.07 & 27.96 & 27.56 & 29.20 & 28.04 & & & \\
\hline
\end{tabular}

a pumping, b recently pumped, e estimated, $h$ tape measurement, $j$ lowest water level recorded, n nearby pumping well 


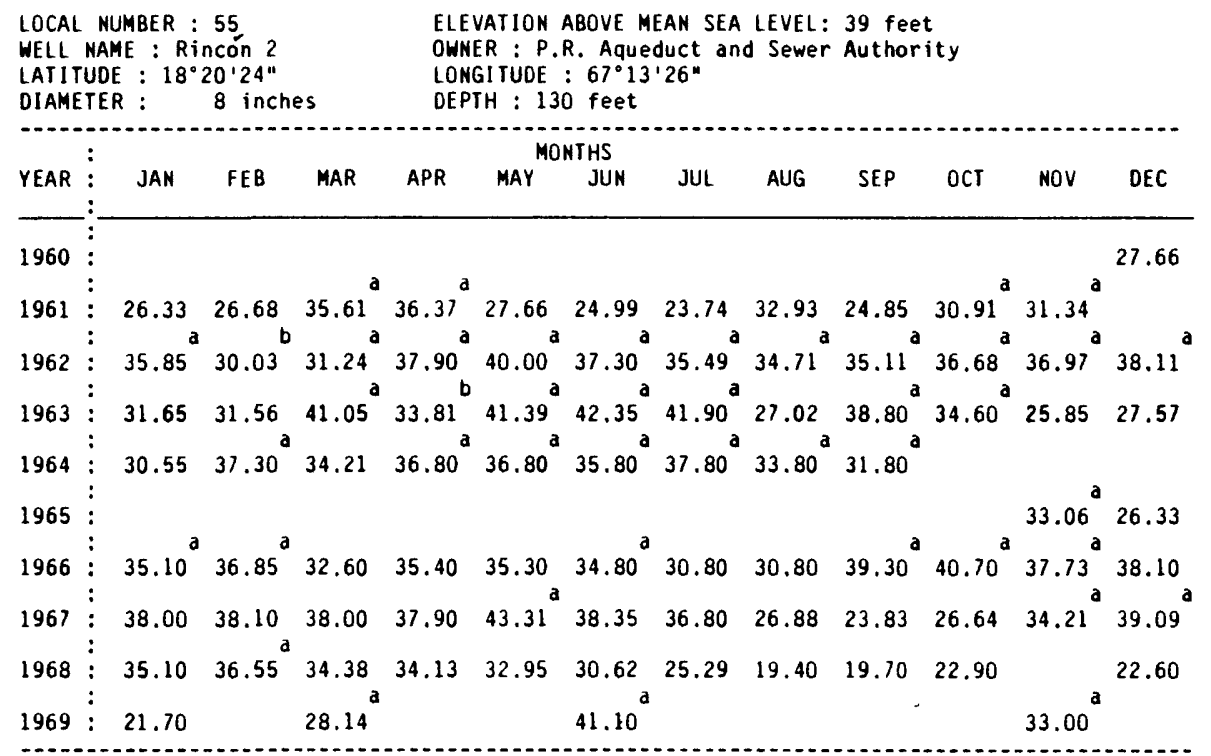

REMARKS: Well discont inued, Decenber, 1969.

a pumping, b recently pumped, e estimated, $h$ tape measurement, $j$ lowest water level recorded, n nearby pumping well 


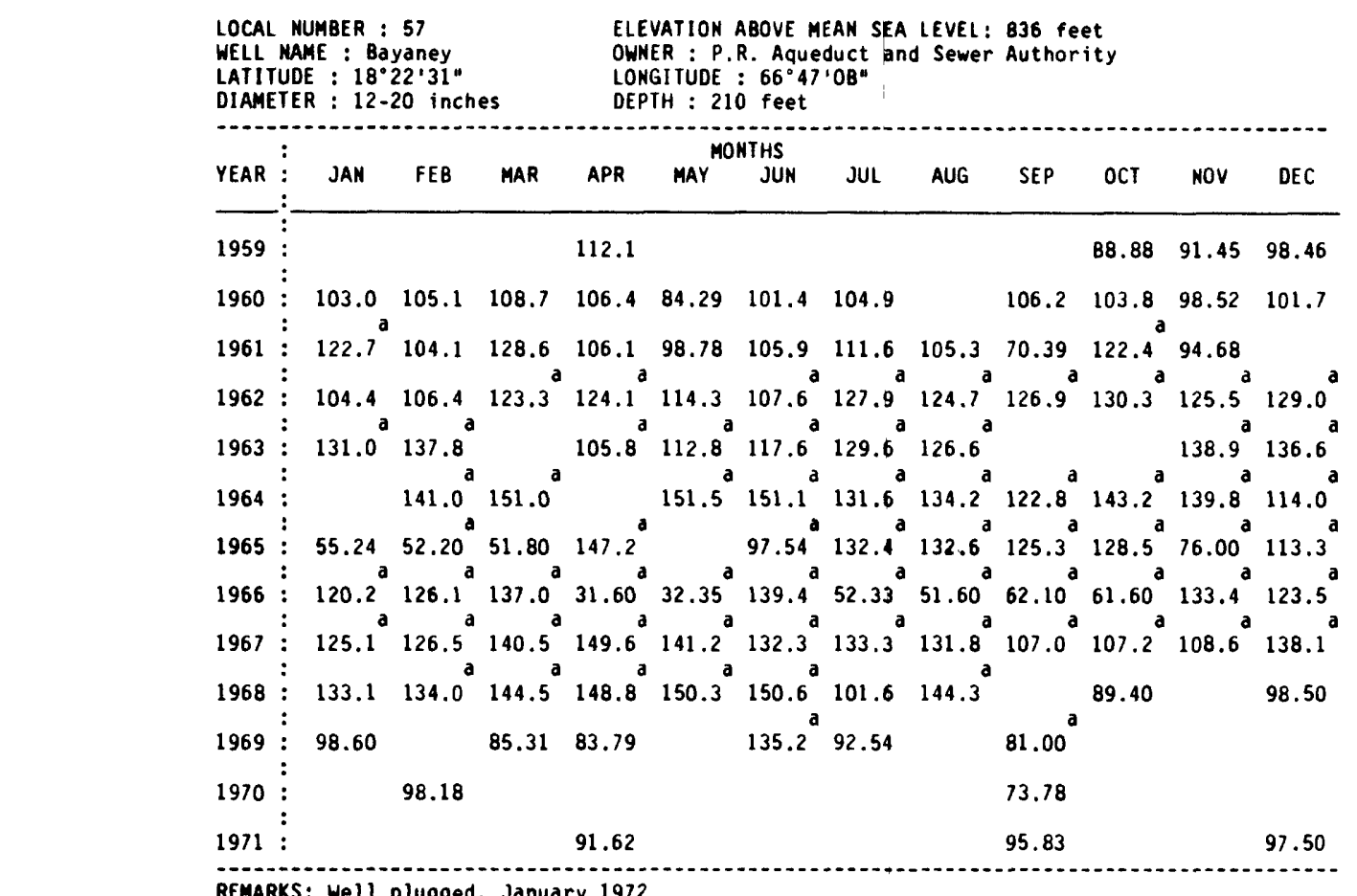

REMARKS: Well plugged, January 1972.

a pumping, b recently pumped, e estimated, $h$ tape measurement, $j$ lowest water level recorded, n nearby pumping well

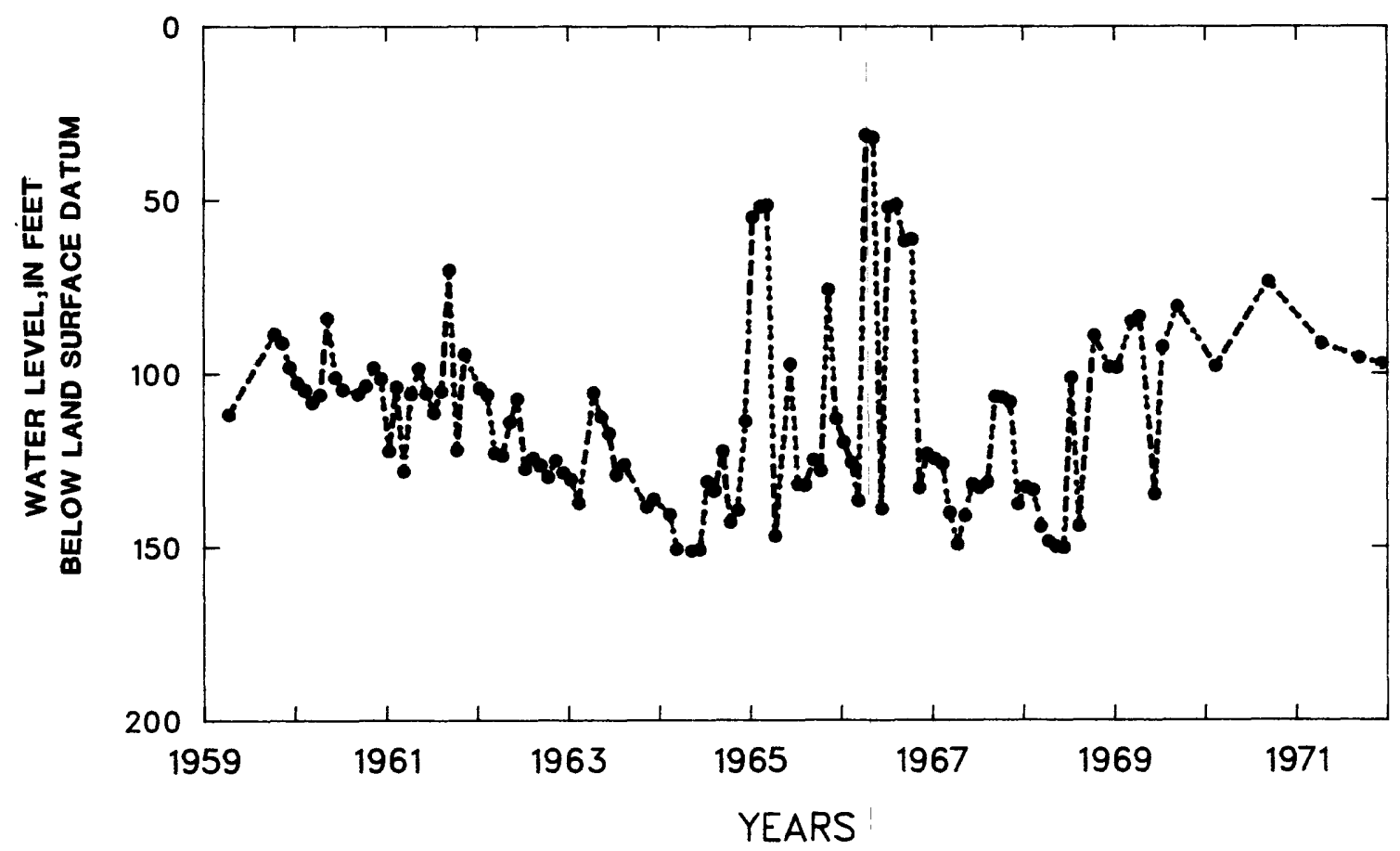

Figure 42.--Gound-water levels at Bayaney, Incal number 57. 
THIS PAGE WAS LEFT BLANK INTENTIONALLY 
WELLS N THE

INTERIOR PROVINCE

Caguas-Juncos Valley

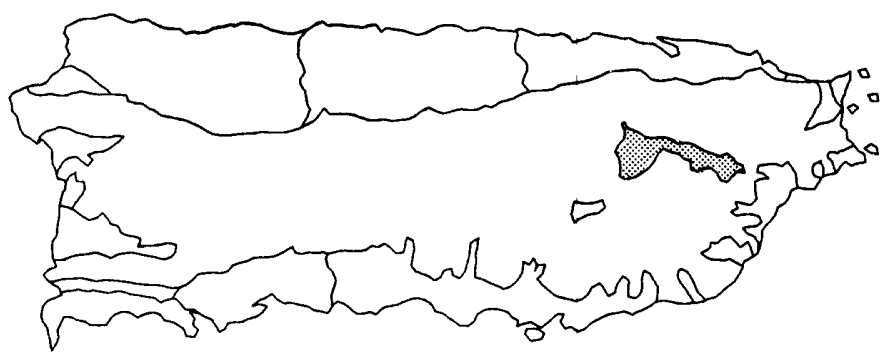

LOCAL MUMBER : 29

WELL NAME : Juncos Well

LATITUDE : $18^{\circ} 02^{\prime} 10^{\prime \prime}$

DIAMETER : 16 inches

ELEVATION ABOVE MEAN SEA LEVEL: 76.2 feet

OWNER : Sucesión Valdivieso

LONGITUDE : $66^{\circ} 43^{\prime} 34^{\circ}$

16 inches DEPTH : 160 feet

\begin{tabular}{|c|c|c|c|c|c|c|c|c|c|c|c|c|}
\hline YEAR & JAN & FEB & -MAR & APR & MAY ${ }^{\text {MO }}$ & $\begin{array}{c}\text { NTHS } \\
\text { JUN }\end{array}$ & JUL & AUG & SEP & OCT & Nov & DEC \\
\hline 1958 & : & & & & & & & $18.49^{a}$ & 16.32 & 17.05 & $13.66^{a}$ & \\
\hline & : & & a & b & & & & & & & & \\
\hline 1959 & : & 20.90 & 28.40 & 15.25 & & 14.40 & & & 10.27 & 14.26 & 15.20 & \\
\hline 1960 & $: 26.34^{a}$ & 14.75 & $27.75^{a}$ & $17.54^{b}$ & 16.70 & 18.01 & 15.33 & 17.28 & 14.15 & 14.63 & 15.44 & 14.78 \\
\hline 1961 & $: 20.40^{\circ}$ & $16.77^{a}$ & $16.10^{\mathrm{a}}$ & $20.44^{a}$ & $21.60^{a}$ & $27.30^{a}$ & $20.40^{\mathrm{a}}$ & 16.89 & 16.25 & 14.00 & 16.09 & \\
\hline 1962 & $: \quad 12.92$ & $19.60^{\mathrm{a}}$ & $16.65^{b}$ & $16.61^{b}$ & $20.40^{b}$ & $22.39^{\mathrm{a}}$ & 19.36 & $17.33^{\mathrm{b}}$ & 17.78 & 17.75 & $20.70^{b}$ & $22.90^{\mathrm{a}}$ \\
\hline 1963 & $: 16.00$ & $21.00^{b}$ & 27.84 & $13.45^{b}$ & $15.28^{b}$ & 15.23 & $14.84^{a}$ & $15.73^{a}$ & 14.31 & 13.03 & 14.37 & $18.12^{\mathrm{a}}$ \\
\hline 1964 & $: \quad 18.80$ & $21.77^{\mathrm{a}}$ & $23.40^{\mathrm{a}}$ & 19.38 & 14.56 & 16.69 & 17.70 & $22.91^{\mathrm{a}}$ & & $18.8^{a}$ & $20.10^{a}$ & \\
\hline 1965 & $: \quad 18.69$ & $29.40^{a}$ & $30.50^{a}$ & 27.42 & $18.80^{a}$ & & $16.75^{3}$ & & 14.32 & $17.12^{a}$ & 14.41 & \\
\hline 1966 & $: \quad 18.30$ & & 18.43 & & & & & & & & & \\
\hline
\end{tabular}

REMARKS: Well discontinued, April 1966.

a pumping, b recently pumped, e estimated, $h$ tape measurement, $j$ lowest water level recorded,

$n$ nearby pumping well 


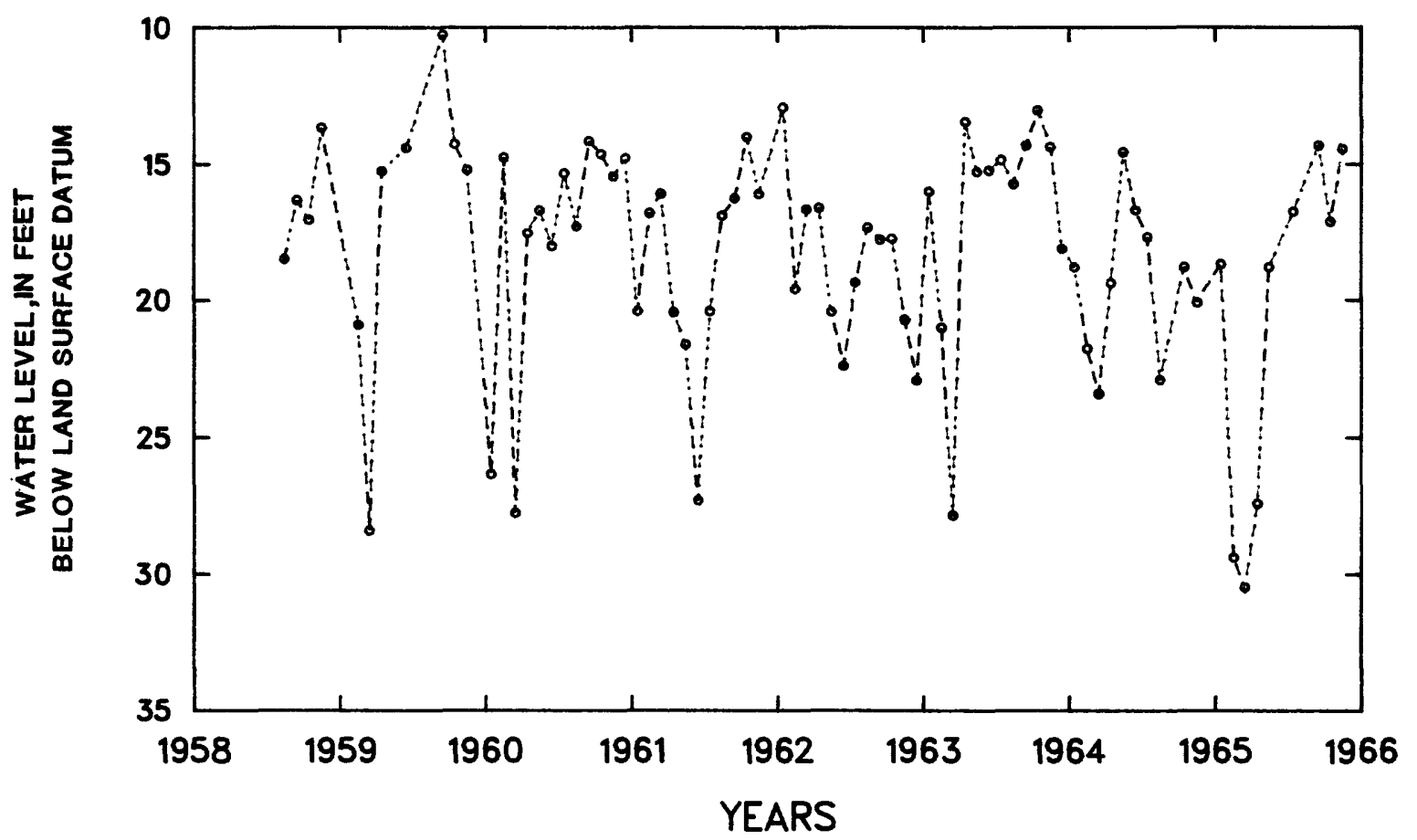

Figure 43.--Ground-water levels at Juncos Well, local number 29. 


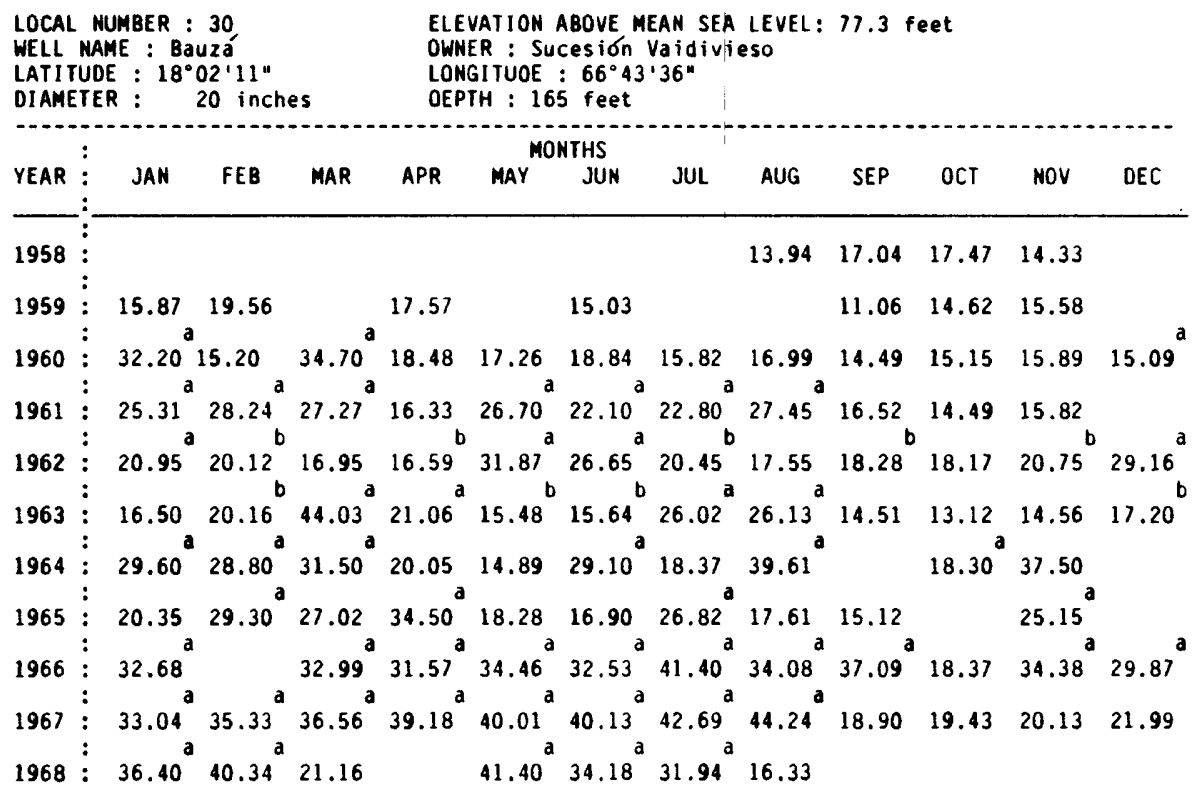

REMARKS: Well discontinued, Septenber 1968.

a pumping, b recently pumped, e estimated, $h$ tape measurement, $j$ lowest water level recorded,

n nearby puaping well 
THIS PAGE WAS LEFT BLANK INTENTIONALLY 


\begin{tabular}{|c|c|c|c|c|c|c|c|c|c|c|c|c|c|}
\hline $\begin{array}{l}\text { LOCAL } \\
\text { WELL } \\
\text { LATIT } \\
\text { DIAME }\end{array}$ & UO & $\begin{array}{l}\text { UMBER : } \\
\text { ME : Gu } \\
E: 18^{\circ} \\
R:\end{array}$ & $\begin{array}{l}50 \\
\text { rabo Ex } \\
15^{\prime} 50^{\prime \prime} \\
13 \text { inch }\end{array}$ & erin & 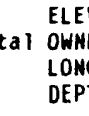 & $\begin{array}{l}\text { VATION } \\
\text { ER : Gu } \\
\text { GI TUDE } \\
\text { TH : } 14\end{array}$ & $\begin{array}{l}\text { ABOVE } \\
\text { rabo AQ } \\
: 65^{\circ} 59 \\
5 \text { feet }\end{array}$ & $\begin{array}{l}\text { EAN SEA } \\
\text { ricultu } \\
\text { "32" }\end{array}$ & $\begin{array}{l}\text { LEVEL } \\
\text { a) St }\end{array}$ & $\begin{array}{r}14 B \\
\text { tion }\end{array}$ & & & \\
\hline & : & & & & & & NTHS & & & & & & \\
\hline YEAR & : & JAN & FEB & MAR & APR & MAY & JUN & JUL & AUG & SEP & $\mathrm{OCT}$ & MOV & $O E C$ \\
\hline 1960 & : & & & & & & & & & & & & 21.89 \\
\hline 1961 & $\vdots$ & 23.76 & & 25.28 & 28.73 & 27.32 & 27.13 & 25.07 & 27.84 & 25.98 & 26.38 & 24.68 & 23.58 \\
\hline 1962 & : & 23.65 & 23.72 & 27.39 & 28.96 & 25.60 & 25.47 & 29.42 & 26.78 & 27.15 & 27.66 & 26.34 & 27.05 \\
\hline 1963 & : & 26.30 & 26.87 & 28.88 & 30.83 & 28.26 & 25.80 & 30.39 & 28.32 & 26.41 & 26.04 & 26.18 & 26.58 \\
\hline 1964 & : & 26.92 & & 30.54 & 30.65 & 30.69 & 30.08 & 27.24 & 27.12 & 27.26 & 26.54 & 26.43 & \\
\hline 1965 & : & & & & 29.83 & 29.52 & 23.61 & 24.23 & & 24.46 & 24.58 & 24.35 & 24.23 \\
\hline 1966 & : & 24.19 & & 23.94 & & 24.10 & & & 26.47 & 25.80 & 25.52 & 25.35 & 25.53 \\
\hline 1967 & : & 24.69 & 26.73 & 27.00 & 31.13 & 32.00 & 32.72 & 30.21 & & 30.16 & 30.12 & 29.75 & \\
\hline 1968 & $\vdots$ & 30.26 & & 30.10 & 29.35 & 30.24 & 29.87 & 25.37 & 24.52 & 24.50 & 24.61 & & 24.80 \\
\hline 1969 & : & & & 26.28 & 27.15 & 23.90 & 26.62 & & 24.50 & 23.34 & & & 25.89 \\
\hline 1970 & : & 26.33 & & 27.10 & 27.67 & 28.11 & & 22.18 & 24.83 & 23.48 & & & \\
\hline 1971 & : & 28.63 & & 28.96 & & 28.53 & & 29.32 & 29.12 & 29.18 & 30.48 & 30.32 & 29.88 \\
\hline 1972 & : & 31.16 & 31.82 & & 31.07 & 32.70 & & & 31.04 & 30.57 & & 29.88 & \\
\hline 1973 & : & 29.98 & & 30.85 & 32.12 & 34.00 & 32.85 & 32.50 & 30.38 & & & & \\
\hline 1974 & : & & & 32.85 & & 34.52 & & & & & & & \\
\hline 1975 & : & & & 32.95 & 33.78 & & 44.38 & & 36.42 & 12.65 & 30.23 & 30.28 & 28.14 \\
\hline 1976 & : & & & & 30.02 & 30.69 & 31.97 & 29.24 & 28.40 & 29.60 & 28,20 & 27.97 & 28.30 \\
\hline 1977 & : & 29.31 & 32.99 & 34.32 & 36.41 & 35.45 & 31.72 & 34.58 & 32.78 & 30.40 & 29.29 & & 31.04 \\
\hline 1978 & : & & 29.41 & 27.28 & 26.28 & 28.20 & 26.50 & 29.02 & 29.67 & 26.51 & 26.99 & 25.60 & \\
\hline 1979 & : & 25.39 & 24.62 & 24.82 & 30.14 & 28.72 & 29.83 & 31.22 & 31.32 & & 30.38 & 30.61 & 30.34 \\
\hline 1980 & $\vdots$ & & 30,44 & 30.80 & 30.40 & 28.93 & 29.02 & 30.43 & 30.79 & 26.89 & 28.96 & 35.29 & 29.68 \\
\hline 1981 & : & 30.36 & 31.28 & & & 30.59 & 29.28 & 28.68 & & 28.77 & 27.94 & 26.90 & 27.88 \\
\hline 1982 & : & 26.97 & 27.01 & 26.97 & 27.13 & 29.13 & 29.06 & 29.85 & 28.75 & 28.64 & 29.05 & 28.53 & 25.93 \\
\hline 1983 & $\vdots$ & 28.19 & 28.14 & 28.19 & 28.25 & 26.18 & 28.85 & 28.81 & 29.78 & 29.57 & 29.83 & 28.99 & 29.55 \\
\hline 1984 & $\vdots$ & 29.44 & 28.86 & 29.17 & 31.34 & 34.33 & 30.15 & 26.96 & 30.20 & 30.45 & 29.86 & 27.35 & 29.40 \\
\hline 1985 & $\vdots$ & 30.62 & 30.34 & 29.74 & & & & & & & & & \\
\hline
\end{tabular}

REMARKS: Well discont inued, April 1985.

a pumping, b recently pumped, e est imated, h tape measurement, j lowest water level recorded,

n nearby pumping well 


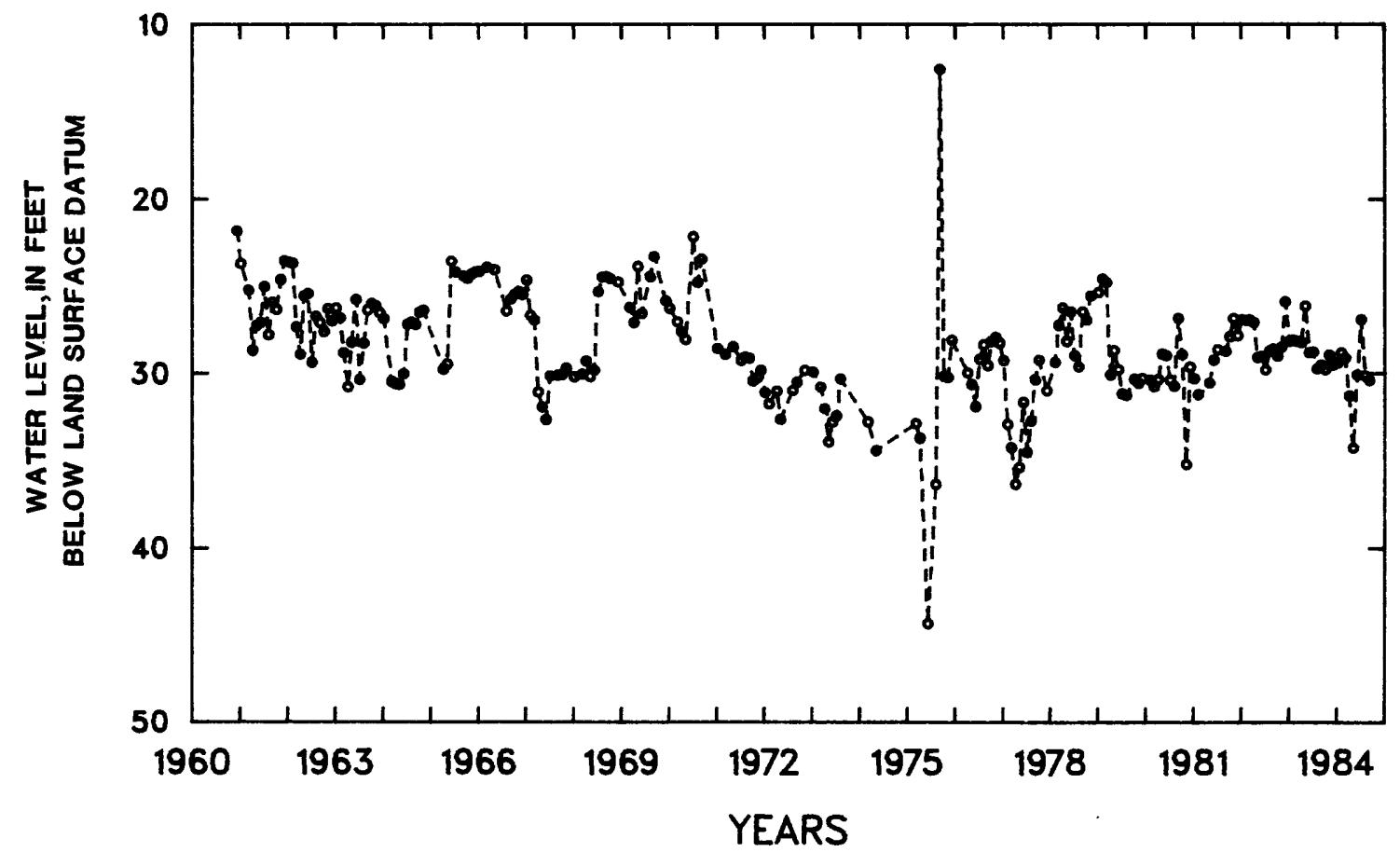

Figure 44.--Ground-water levels at Gurabo Experimental Station, local number 50 . 


\begin{tabular}{|c|c|c|c|c|c|c|c|c|c|c|c|c|}
\hline $\begin{array}{l}\text { LOCAL } \\
\text { WELL } \\
\text { LATIT } \\
\text { DIAME }\end{array}$ & $\begin{array}{l}\text { NUMBER } \\
\text { NAME : } \\
\text { UDE : } 1 \mathrm{~B}^{\circ} \\
\text { TER : }\end{array}$ & $\begin{array}{l}51 \\
15 \text { well } \\
15 \cdot 08 " \\
10 \text { inch }\end{array}$ & & $\begin{array}{l}\text { EL } \\
\text { OW } \\
\text { LO } \\
\text { DE }\end{array}$ & $\begin{array}{l}\text { VATION } \\
\text { ER : GU } \\
\text { GITUDE } \\
\text { TH : } 27\end{array}$ & $\begin{array}{l}\text { ABove } \\
\text { rabo Ex } \\
: 65^{\circ} 59 \\
0 \text { feet }\end{array}$ & $\begin{array}{l}\text { EAN SE } \\
\text { perime } \\
24 "\end{array}$ & $\begin{array}{l}\text { LEVEI } \\
\text { al St }\end{array}$ & $\begin{array}{l}197.4 \\
\text { tion }\end{array}$ & feet & & \\
\hline$\ldots$ & : & & & & MO & NTHS & & & & & & 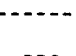 \\
\hline YEAR & JAN & FEB & MAR & APR & MAY & JUH & JUL & AUG & SEP & OCT & NOV & DEC \\
\hline 1960 & : & & & & & & & & & & & 11.13 \\
\hline 1961 & $: \quad 13.68$ & & 14.58 & 15.04 & 14.72 & 14.87 & 14.78 & 14.93 & 13.93 & 13.54 & 13.19 & 13.50 \\
\hline 1962 & $: \quad 13.94$ & 14.24 & 14.92 & 14.81 & 13.87 & 13.80 & 14.01 & 14.37 & 13.70 & 14.24 & 15.00 & 14.27 \\
\hline 1963 & 15.18 & 15.37 & 15.98 & 15.60 & 14.39 & 15.60 & 14.43 & 14.43 & 14.69 & 14.95 & 15.05 & 15.73 \\
\hline
\end{tabular}

a pumping, b recently pumed, e estimated, $h$ tape measurement, $j$ lowest water level recorded, n nearby pumping well

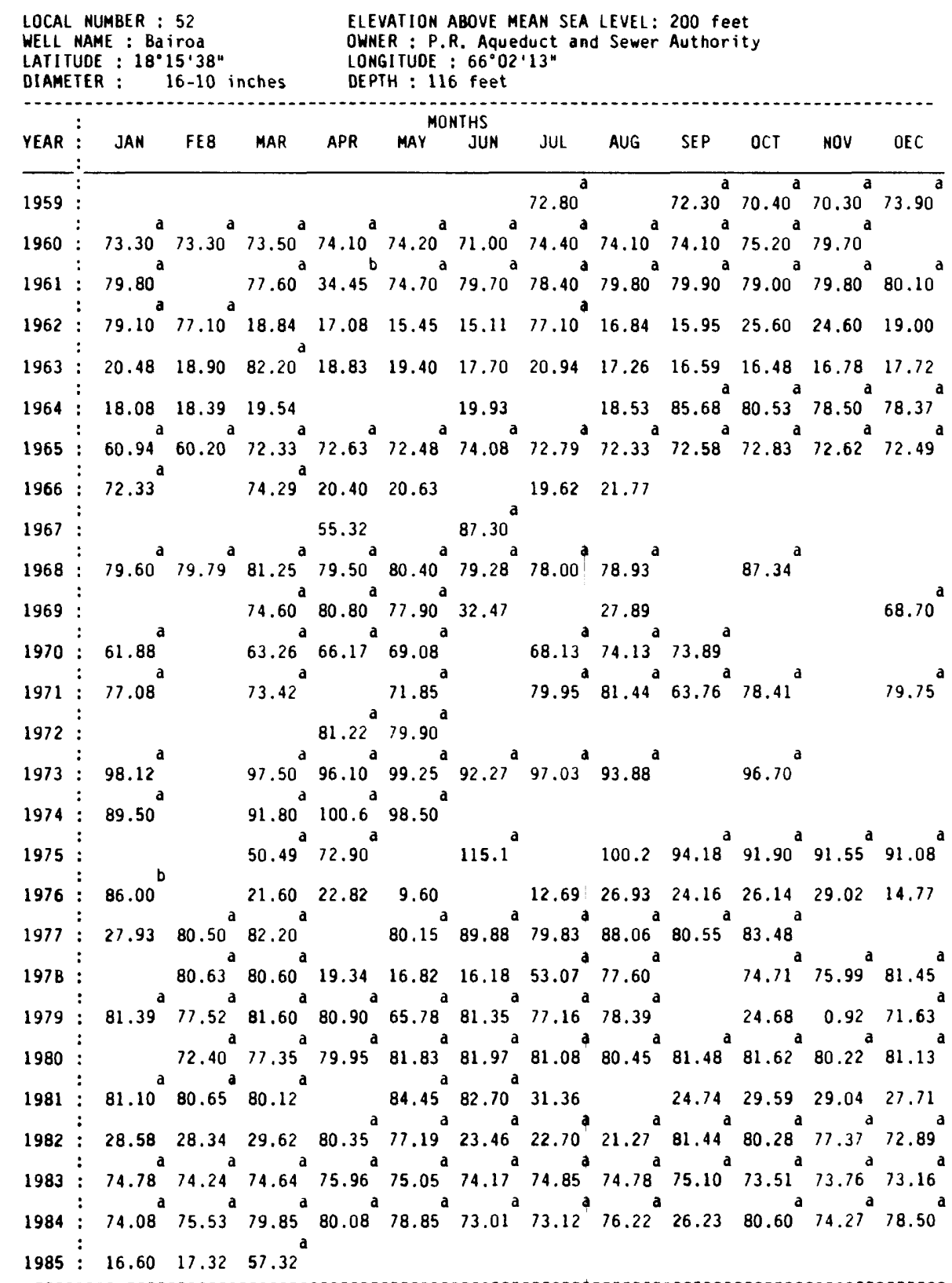

REMARKS: Well discontinued, April 1985.

a pumping, b recently pumped, e estimated, $h$ tape measurement, $j$ lowest water level recorded. n nearby pumping well 


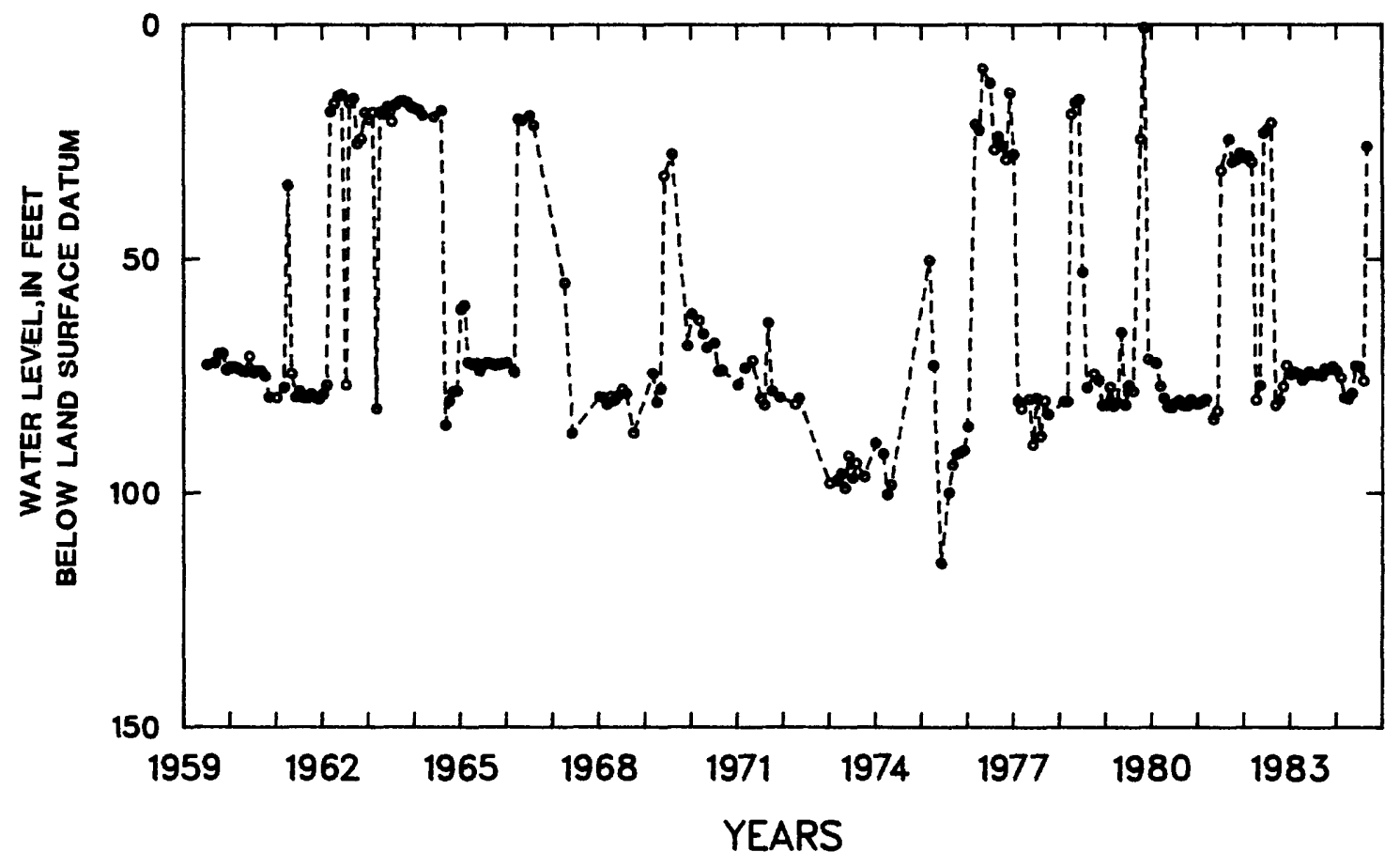

Figure 45.--Ground-water levels at Bairoa,

local number 52. 
NELLS IN THE

INTERIOR PROVINCE

Cayey Valley

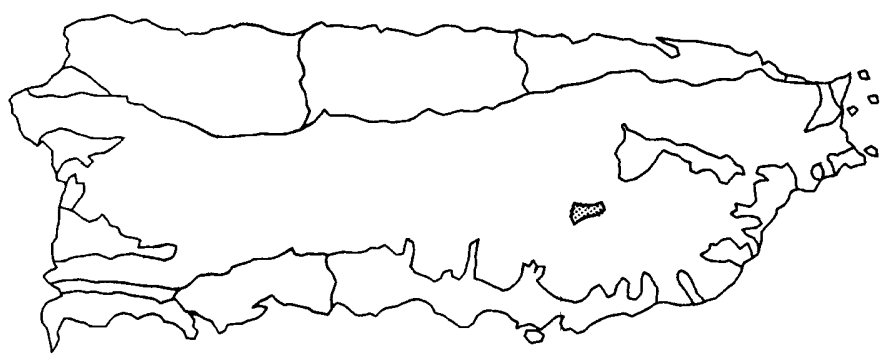

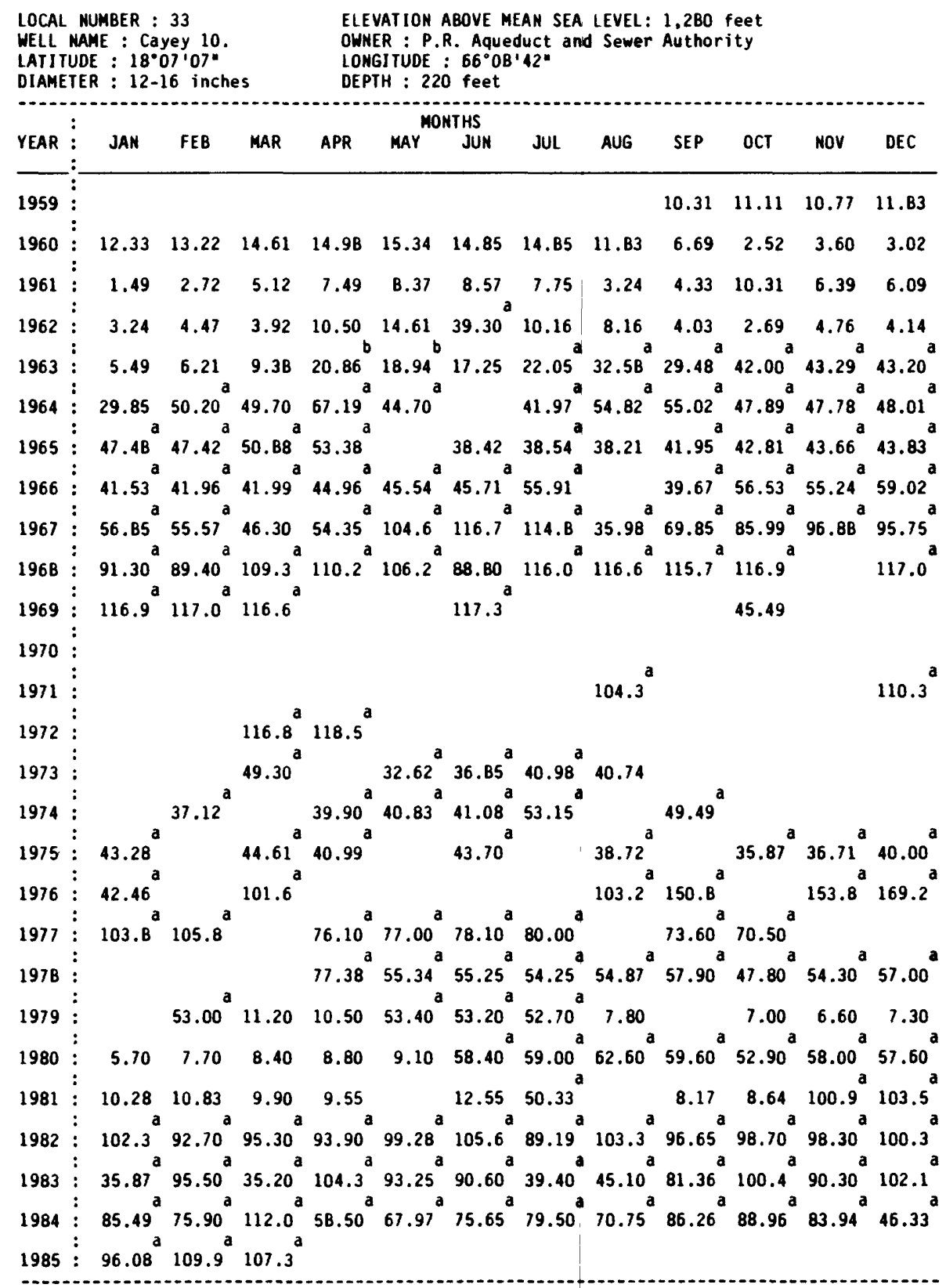

REMARKS: Well discontinued, April 1985.

a pumping, b recently pumped, e estimated, $h$ tape measurement, $j$ lowest water level recorded,

n nearby pumping well 


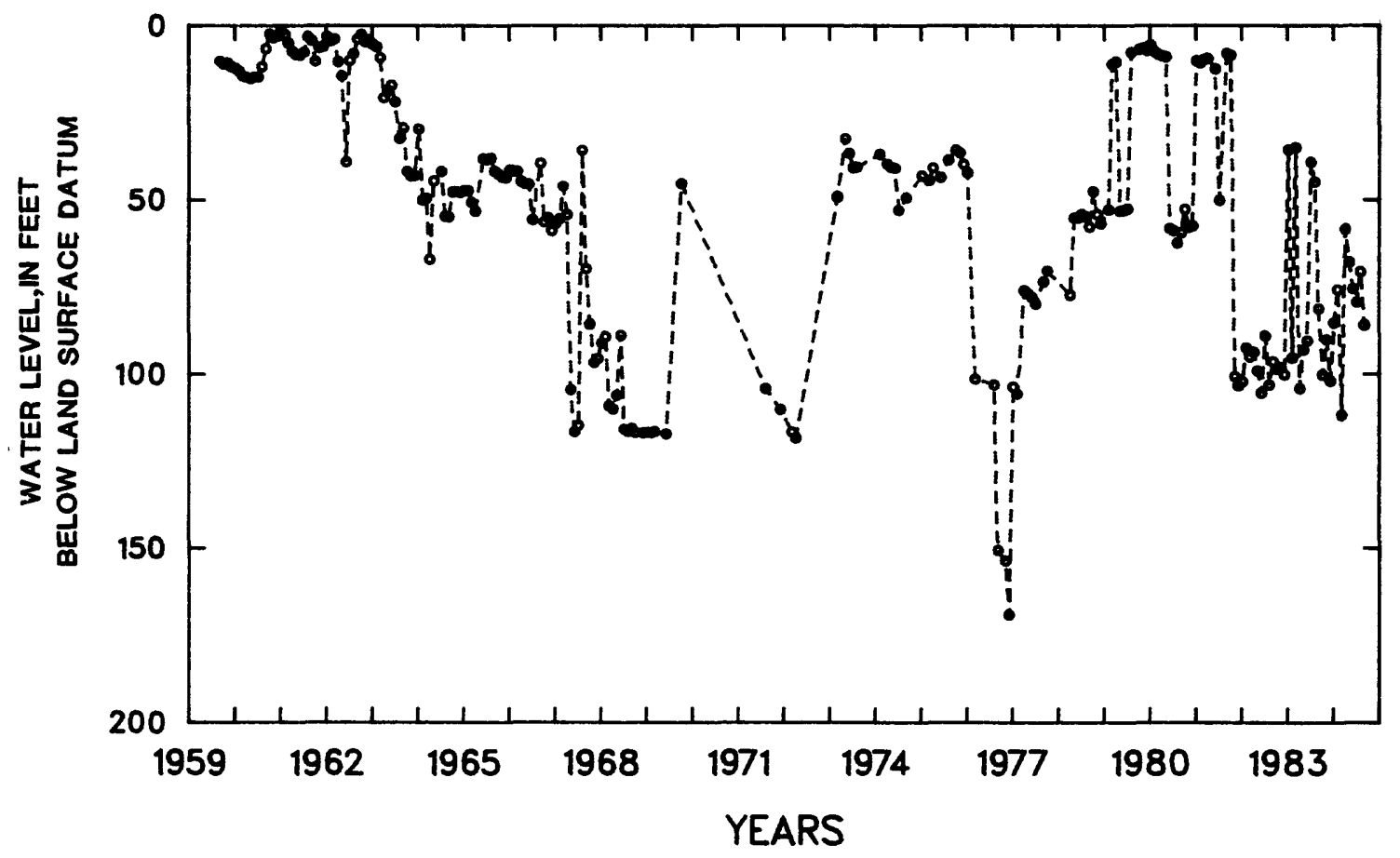

Figure 46.--Ground-water levels at Cayey 10, local number 33. 


\begin{tabular}{|c|c|c|c|c|c|c|c|c|c|c|c|c|}
\hline \multicolumn{4}{|c|}{$\begin{array}{l}\text { LOCAL NUMBER : } 34 \\
\text { WELL NAME : Cayey } 9 \\
\text { LATI IUDE : } 18^{\circ} 07 \cdot 41^{\prime \prime} \\
\text { OIAMETER : } 12-16 \text { inches }\end{array}$} & \multicolumn{7}{|c|}{$\begin{array}{l}\text { ELEVATION ABOVE MEAN SEA LEVEL: } 1,214 \text { feet } \\
\text { OWNER : P.R. AqueduCt and Sewer Authority } \\
\text { LONGITUDE : } 66^{\circ} 08^{\prime} 07^{\prime \prime} \\
\text { DEPTH : } 167 \text { feet }\end{array}$} & \multirow[b]{3}{*}{ NOV } & \multirow[b]{3}{*}{$\mathrm{DEC}$} \\
\hline & & & & \multicolumn{4}{|c|}{ MONTHS } & \multirow[b]{2}{*}{ AUG } & \multirow[b]{2}{*}{ SEP } & \multirow[b]{2}{*}{ OCT } & & \\
\hline YEAR: & JAN & FEB & MAR & APR & MAY & JUN & JUL & & & & & \\
\hline $1959:$ & & & & & & & & & 9.36 & 9.32 & 9.38 & 9.63 \\
\hline $1960:$ & 9.95 & 10.01 & 10.35 & 9.98 & 9.37 & 9.18 & 9.30 & 7.28 & 7.09 & 7.88 & 8.27 & 7.63 \\
\hline 1961 & 7.79 & 7.47 & 9.21 & 9.42 & 8.17 & B. 45 & 6.24 & 7.57 & 7.72 & 6.81 & 7.41 & 7.07 \\
\hline $1962:$ & 7.44 & 7.73 & 8.19 & 7.90 & 7.63 & B.67 & 6.80 & 7.94 & 7.09 & 7.95 & 8.20 & 8.55 \\
\hline $1963:$ & 8.60 & 8.55 & 8.83 & 8.91 & 8.94 & 7.90 & 8.55 & 8.68 & 8.33 & 8.32 & 8.62 & 9.09 \\
\hline 1964 & 9.17 & 9.30 & 9.39 & 9.54 & 9.38 & & 8.75 & 8.50 & 9.09 & 7.86 & 9.06 & 8.99 \\
\hline $1965:$ & & & $44.99^{\mathrm{a}}$ & $50.99^{a}$ & $50.57^{a}$ & 8.26 & $17.97^{\mathrm{a}}$ & $17.47^{a}$ & $27.96^{a}$ & $28.64^{\mathrm{a}}$ & $43.11^{a}$ & 43.02 \\
\hline $1966:$ & $41.02^{a}$ & $49.13^{a}$ & $54.59^{\mathrm{a}}$ & $50.58^{a}$ & $51.48^{a}$ & $51.60^{\mathrm{a}}$ & $51.73^{a}$ & & $42.43^{a}$ & 8.70 & $41.69^{a}$ & $48.85^{a}$ \\
\hline $1967:$ & $42.50^{a}$ & 15.61 & $56.55^{\mathrm{a}}$ & $55.78^{a}$ & $99.48^{\mathrm{a}}$ & $97.40^{\mathrm{a}}$ & $108.5^{a}$ & $54.85^{\mathrm{a}}$ & $61.17^{a}$ & $56.78^{a}$ & $56.70^{a}$ & 15.57 \\
\hline $1968:$ & 13.79 & 11.41 & 10.93 & 11.26 & $78.50^{\mathrm{a}}$ & 10.88 & 10.34 & 9.47 & & & & \\
\hline
\end{tabular}

REMARKS: Well discont inued, September 1968.

a pumping, b recently pumped, e estimated, $h$ tape measurement, $j$ lowest water level recorded,

n nearby pumping well

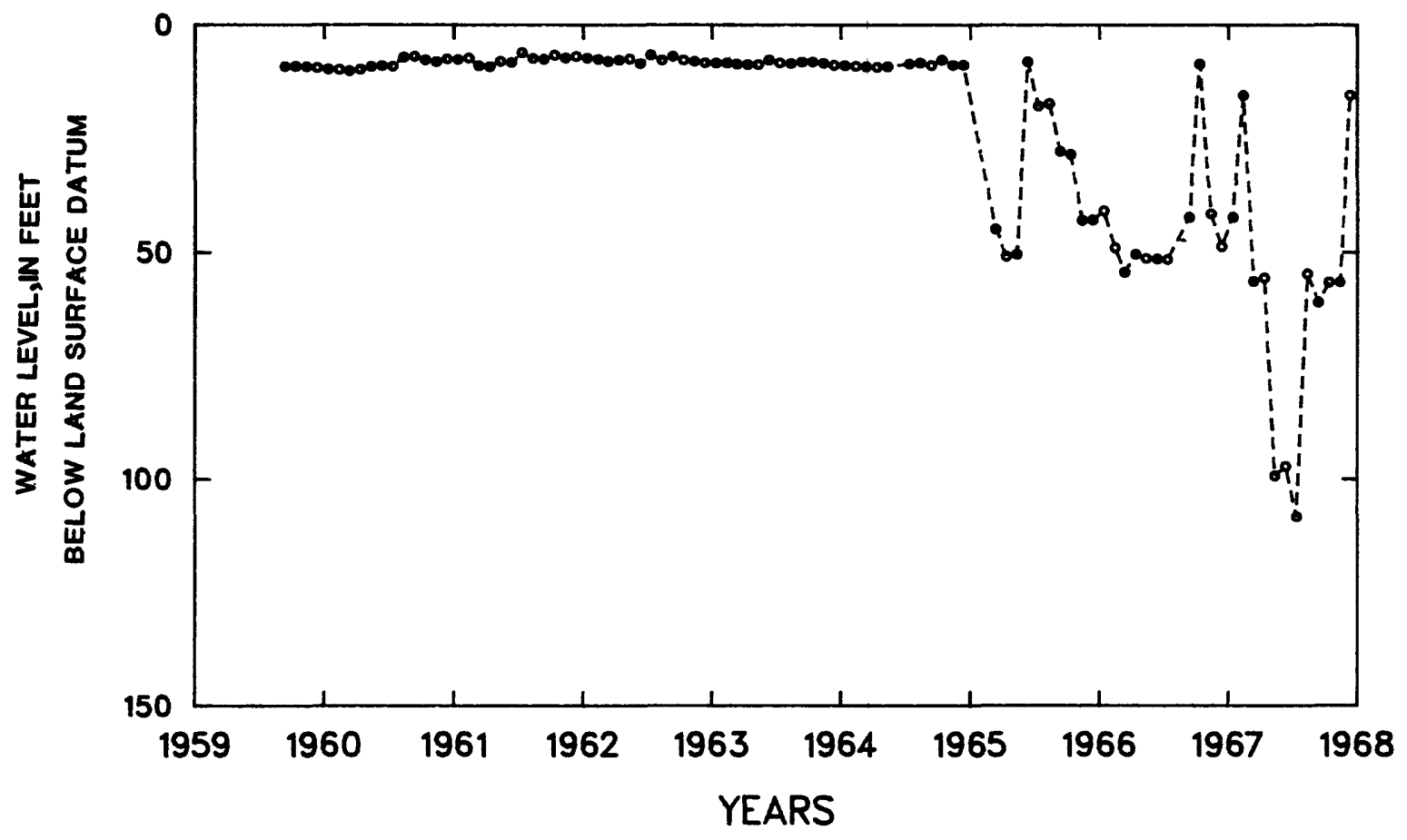

Figure 47.--Ground-water levels at Cayey 9. local number 34. 


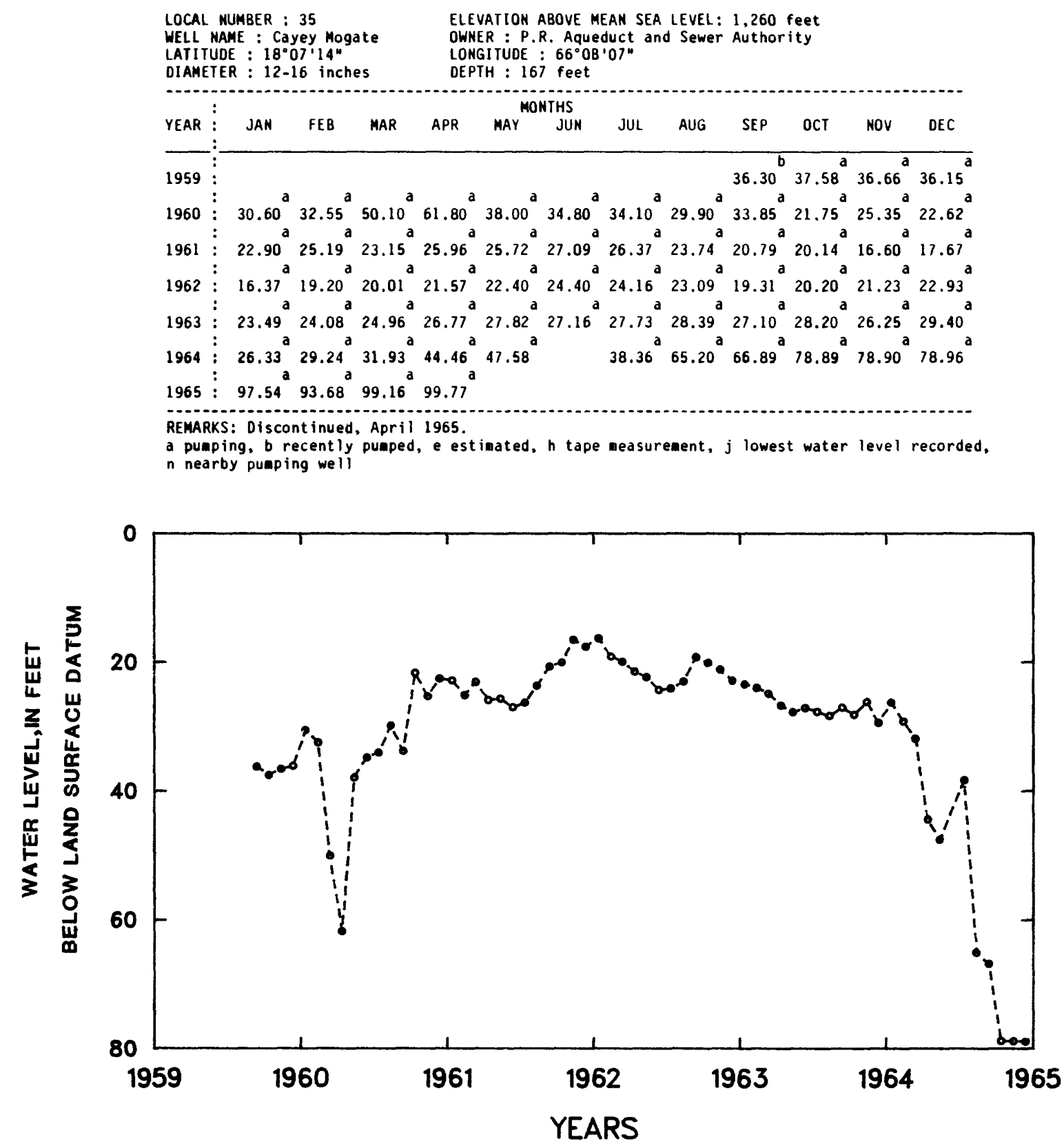

Figure 48.--Ground-water levels at Cayey Mogate, local number 35. 


\section{SELECTED REFERENCES}

Colón-Dieppa, Eloy, Díaz, P.L., and García, René, 1987, Water resources data Puerto Rico and the U.S. Virgin Islands, Water Year 1985: U.S. Geological Survey Water-Data Report PR-85-1, 400 p.

Curtis, R.E., Jr., Aquino, Zaida, Díax, P.L., and García, René, 1988, Water-resources data Puerto Rico and the U.S. Virgin Islands, Water Year 1986: U.S. Geological Survey Water-Data Report PR-86-1, 362 p.

Curtis, R.E., Jr., Aquino, Zaida, Díaz, P.L, and Vachier, Ricardo, 1989, Water resources data Puerto Rico and the U.S. Virgin Islands, Water Year 1987: U.S. Geological Survey Water-Data Report PR-87-1, 356 p. 1989, Water resources data for Puerto Rico and the U.S. Virgin Islands, Water Year 1988: U.S. Geological Survey Water-Data Report PR-88-1, 460 p.

1990, Water resources data for Puerto Rico and the U.S. Virgin Islands, Water Year 1989: U.S. Geological Survey Water-Data Report PR-89-1, 419 p.

Curtis, R.E., Jr., Guzmán-Ríos, Senén, and Díaz, P.L., 1984, Water-resources data Puerto Rico and the U.S. Virgin Islands, Water Year 1983: U.S. Geological Survey Water-Data Report PR-83-1, 302 p. 1985, Water resources data Puerto Rico and the U.S. Virgin Islands, Water Year 1984: U.S. Geological Survey Water-Data Report PR-84-1, 374 p.

Curtis, R.E., Jr., McKinley P.W., and Colón-Ramos, H.M., 1983, Water resources data Puerto Rico, Water Years 1981-82: U.S. Geological Survey Water-Data Report PR-81-82-1, 414 p.

Kipple, F.P., and others, 1968, Water records of Puerto Rico, 1958-63: U.S. Geological Survey Water-Data Report, 353 p.

Rickler, J.G., and others, 1970, Water records of Puerto Rico, 1964-67, Volume 1, north and northeast slopes: U.S. Geological Survey Water-Data Report 71-238, 265 p.

1970, Water records of Puerto Rico, 1964-67, Volume 2, south and west slopes: U.S. Geological Survey Water-Data Report, 308 p.

Robison, T.M., and others, 1972, Water records of the U.S. Virgin Islands, 1962-69: U.S. Geological Survey Water-Data Report, 163 p.

Snyder P.J., 1982, Map Projections Used by the U.S. Geological Survey: U.S. Geological Survey Miscellaneous Investigations Map Series I-1402, 61 p.

U.S. Geological Survey, 1973, Water resources data for Puerto Rico, 1968, Part 1, Surface water records: U.S. Geological Survey Water Data-Report, 87 p.

1973, Water resources data for Puerto Rico, 1968, Part 2, Surface water records: U.S. Geological Survey Water-Data Report, 87 p.

1973, Water resources data for Puerto Rico, 1968-72, Part 3, Ground water records: U.S. Geological Survey Water-Data Report, 78 p.

1973, Water resources data for Puerto Rico, 1969, Part 1, Surface water records: U.S. Geological Survey Water-Data Report, 89 p. 


\section{SELECTED REFERENCES--Continued}

1973, Water resources data for Puerto Rico, 1970, Part 1, Surface water records: U.S. Geological Survey Water-Data Report, 90 p.

1973, Water resources data for Puerto Rico, 1971, Part 1, Surface water records: U.S. Geological Survey Water-Data Report, 72 p.

1974, Water resources data for Puerto Rico, 1968-72, Part 2A, Water quality records: U.S. Geological Survey Water-Data Report, 410 p.

1974, Water resources data for Puerto Rico, 1972, Part 1, Surface water records: U.S. Geological Survey Water-Data Report, 70 p.

1975, Water resources data for Puerto Rico, 1968-72, Part 2B, Water quality records (sediment): U.S. Geological Survey Water-Data Report, 182 p.

1975, Water resources data for Puerto Rico, 1973, Part 1, Surface water records: U.S. Geological Survey Water-Data Report, 69 p.

1975, Water resources data for Puerto Rico, 1973, Part 2, Water quality records: U.S. Geological Survey Water-Data Report, 133 p.

1976, Water resources data for Puerto Rico, 1974, Part 1, Surface water records: U.S. Geological Survey Water-Data Report, 66 p.

1976, Water resources data for Puerto Rico, 1974, Part 2, Water quality records: U.S. Geological Survey Water-Data Report, 158 p.

1978, Water resources data for Puerto Rico, Surface and quality-of-water records, water years 1975-76, Ground water records, water years 1973-76: U.S. Geological Survey Water- Data Report-PR-76-1, 366 p. 1979, Water-Resources Investigations in Puerto Rico and the U.S. Virgin Islands, 1978: U.S. Geological Survey Folder.

1980, Water resources data for Puerto Rico, Surface and quality-of-water records, water year 1977, Ground water records, water year 1977: U.S. Geological Survey Water-Data Report PR-77-1, 215 p.

1981, Water resources data for Puerto Rico, Water Year 1978: U.S. Geological Survey Water-Data Report PR-78-1, 255 p.

1982, Water resources data for Puerto Rico, Water Years 1979-80: U.S. Geological Survey Water-Data Report PR-79-80, 408 p. 